Prepared for Physics of Elementary Particles and Atomic Nuclei. Theory

\title{
Possible studies at the first stage of the NICA collider operation with polarized and unpolarized proton and deuteron beams
}

V.V.Abramov ${ }^{1}$, A.Aleshko $^{2}$, V.A. Baskov ${ }^{3}$, E. Boos ${ }^{2}$,

V. Bunichev ${ }^{2}$, O.D. Dalkarov ${ }^{3}$, R. El-Kholy ${ }^{4}, A$. Galoyan $^{5}$, A.V. Guskov ${ }^{6}$, V.T. Kim ${ }^{7,8}$,E. Kokoulina ${ }^{5,9}$, I.A. Koop ${ }^{10,11,12}$, B.F. Kostenko ${ }^{13}$,

A.D. Kovalenko ${ }^{5}$, V.P. Ladygin ${ }^{5}, A . B$. Larionov $^{14,15}$, A.I. L'vov ${ }^{3}$, A.I. Milstein ${ }^{10,11}$, V.A. Nikitin ${ }^{5}$ N. N. Nikolaev ${ }^{16,26}$, A.S. Popov ${ }^{10}$, V.V. Polyanskiy ${ }^{3}$

J.-M. Richard ${ }^{17}$, S. G. Salnikov ${ }^{10}$, 'A.A.Shavrin ${ }^{18}$ ', P.Yu. Shatunov ${ }^{10,11}$, Yu.M. Shatunov ${ }^{10,11}$, O.V.Selyugin ${ }^{14}$, M. Strikman ${ }^{19,}$, E. Tomasi-Gustafsson ${ }^{20}$, V.V. Uzhinsky ${ }^{13,}$, Yu.N. Uzikov ${ }^{6,21,22, *}$, Qian Wang ${ }^{23}$, Qiang Zhao ${ }^{24,25}$, A.V. Zelenov ${ }^{7}$

${ }^{1}$ NRC "Kurchatov Institute" - IHEP, Protvino 142281, Moscow region, Russia

${ }^{2}$ Skobeltsyn Institute of Nuclear Physics, MSU, Moscow, 119991 Russia

${ }^{3}$ P.N. Lebedev Physical Institute,Leninsky prospect 53, 119991 Moscow, Russia

${ }^{4}$ Astronomy Department, Faculty of Science, Cairo University, Giza, Egypt, 12613

${ }^{5}$ Veksler and Baldin Laboratory of High Energy Physics, Joint Institute for Nuclear Research,Dubna, Moscow region, 141980 Russia

${ }^{6}$ Joint Institute for Nuclear Researches, DLNP, Dubna, Moscow reg. 141980 Russia

${ }^{7}$ Petersburg Nuclear Physics Institute NRC KI, Gatchina, Russia

${ }^{8}$ St. Petersburg Polytechnic University, St. Peterburg, Russia

${ }^{9}$ Sukhoi State Technical University of Gomel, Prospect Octiabria, 48, 246746 Gomel, Belarus

10 Budker Institute of Nuclear Physics of SB RAS, 630090 Novosibirsk, Russia

${ }^{11}$ Novosibirsk State University, 630090 Novosibirsk, Russia

12 Novosibirsk State Technical University,630092 Novosibirsk, Russia

\footnotetext{
*E-mail: uzikov@jinr.ru; Corresponding author
} 
${ }^{13}$ Laboratory of Information Technologies, Joint Institute for Nuclear Research, Dubna, Moscow region, 141980 Russia

${ }^{14}$ Joint Institute for Nuclear Researches, BLTP, Dubna, Moscow reg. 141980 Russia

${ }^{15}$ Institut für Theoretische Physik, Justus-Liebig-Universität, 35392 Giessen, Germany

${ }^{16}$ L.D. Landau Institute for Theoretical Physics, 142432 Chernogolovka, Russia

${ }^{17}$ Université de Lyon, Institut de Physique des 2 Infinis de Lyon, UCBL-IN2P3-CNRS, 4, rue Enrico Fermi, Villeurbanne, France

${ }^{18}$ St. Petersburg State University, St. Peterburg, Russia

${ }^{19}$ Pensilvania State University, 104 Davey Laboratory University Park PA 16802 USA

${ }^{20}$ DPhN, IRFU, CEA, Université Paris-Saclay, 91191 Gif-sur-Yvette Cedex, France

${ }^{21}$ Dubna State University, Dubna, Moscow reg. 141980 Russia

22 Department of Physics, M.V. Lomonosov State University, Moscow, 119991 Russia

${ }^{23}$ Guangdong Provincial Key Laboratory of Nuclear Science, Institute of Quantum Matter, South China Normal University, Guangzhou 510006, P.R. China

${ }^{24}$ Institute of High Energy Physics, Chinese Academy of Sciences, Beijing 100049, P.R. China

${ }^{25}$ University of Chinese Academy of Sciences, Beijing 100049, P.R. China

26 Moscow Institute of Physics and Technology (National Research University), 141701

Dolgoprudny, Russia

Nuclotron based Ion Collider fAcility (NICA) project is in progress at the Joint Institute for Nuclear Research and will start experiments with heavy ions. In the context of the NICA Hadronic Physics programme double polarized $p p$-, $d d$ - and $p d$ - collisions even at lower energies of $\sqrt{s_{N N}}=3.4-10 \mathrm{GeV}$, which will be accessible already at the initial stage of experiments,are essential tools for precise understanding the spin dependence of the nucleon-nucleon strong interactions, in both elastic and deep-inelastic regimes. A special interest is interaction in few baryon systems at double strangeness, charm and beauty thresholds.For instance, polarized large-angle elastic $p p$ and $p n$ scattering near the charm threshold allows one to get an access to properties of possible exotic multiquark states and their relation to the states recently observed at LHCb.Large angle scattering of protons and deuterons on the deuteron contains unique information on the short-range structure of the deuteron, its non-nucleonic degrees of freedom and also on color transparency phenomenon. Furthermore, double polarized proton-deuteron scattering offer a possibility to test the Standard Model through the search for time-invariance (or CP- invariance under CPT symmetry) violation and parity-violation in single-polarized scattering. This paper contains suggestions for experiments with usage of the Spin Physics Detector (SPD) and discusses perspectives of the first stage of the SPD Programme. This includes experiments with non-polarized beams too as well as collisions like ${ }^{12} \mathrm{C}-{ }^{12} \mathrm{C}$ and ${ }^{40} \mathrm{Ca}$ ${ }^{40} \mathrm{Ca}$. 


\section{Contents}

1 The SPD setup and experimental conditions ${ }^{1} \quad 7$

2 Elastic $p N, p d$ and $d d$ scattering ${ }^{2} \quad \mathbf{8}$

2.1 Spin amplitudes of $p N$ elastic scattering . . . . . . . . . . . . 8

2.2 Polarized $p d$ elastic diffraction scattering within the Glauber model . . . . 9

2.3 Quasielastic pd-scattering $p+d \rightarrow\{p p\}\left({ }^{1} S_{0}\right)+n \ldots \ldots . \ldots 11$

2.4 Elastic $d d$ scattering . . . . . . . . . . . . . . . . . . . 12

2.5 Double polarized large angle $p N$ elastic scattering . . . . . . . . . . . 13

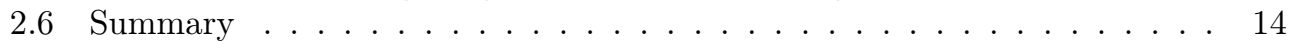

3 Studying periphery of the nucleon in diffractive $p p$ scattering ${ }^{3} \quad 15$

4 Hadron structure and spin effects in elastic hadron scattering at NICA $\begin{array}{ll}\text { energies }{ }^{4} & 18\end{array}$

4.1 HEGS model and spin effects in the dip region of momentum transfer . . 19

4.2 Conclusions . . . . . . . . . . . . . . . . . . . . 23

5 Single-spin physics ${ }^{5} \quad \mathbf{2 5}$

5.1 Model of chromomagnetic polarization of quarks . . . . . . . . . . . 27

5.2 Single-spin hadron asymmetry . . . . . . . . . . . . . . . . 31

5.3 Transverse polarization of hyperons . . . . . . . . . . . . . . . 33

6 Vector light and charmed meson production ${ }^{6} \quad \mathbf{3 7}$

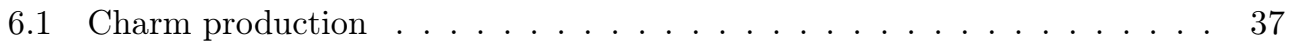

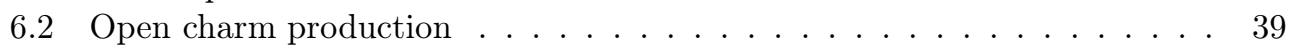

6.3 Backward meson production . . . . . . . . . . . . . . . . 41

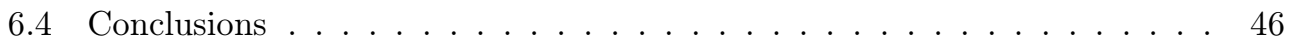

$\begin{array}{lll}7 & \text { Exclusive hard processes with deuteron at } \mathrm{NICA}^{7} & 48\end{array}$

7.1 Probing dynamics of nucleon - nucleon interaction in proton - deuteron quasielastic scattering . . . . . . . . . . . . . . . . . . 48

7.2 Probing microscopic deuteron structure . . . . . . . . . . . . . 49

8 Scaling behaviour of exclusive reactions with lightest nuclei and spin observables $^{8}$

\footnotetext{
${ }^{1}$ This section is written by A.V. Guskov (E-mail: alexey.guskov@cern.ch) and A.D. Kovalenko (Email:kovalen@dubna.ru)

${ }^{2}$ This section is written by Yu.N. Uzikov; E-mail:uzikov@jinr.ru

${ }^{3}$ This section is written by V.A. Baskov, O.D. Dalkarov, A.I. L'vov (E-mail: lvov@x4u.lebedev.ru) and V.V. Polyanskiy

${ }^{4}$ This section is written by O.V. Selyugin; E-mail: selugin@theor.jinr.ru

${ }^{5}$ This section is written by V. Abramov; E-mail: Victor.Abramov@ihep.ru

${ }^{6}$ This section is written by E. Tomasi-Gustafsson; E-mail: egle.tomasi@cea.fr.

${ }^{7}$ This section is written by M. Strikman; E-mail: mxs43@psu.edu

${ }^{8}$ This section is written by V.P. Ladygin (E-mail: vladygin@jinr.ru) and Yu.N. Uzikov
} 
9 Multiquark correlations and exotic hadron state production ${ }^{9} \quad \mathbf{5 4}$

9.1 Multiquark correlations and exotic state production at SPD NICA . . . 54

9.2 Multiquark correlations: fluctons in nuclei . . . . . . . . . . . . . 54

9.3 Few-quark correlations: Diquarks . . . . . . . . . . . . . . . . . 55

9.4 Multiparton scattering . . . . . . . . . . . . . . . . . . . 56

9.5 Multiquark exotic state production . . . . . . . . . . . . . 57

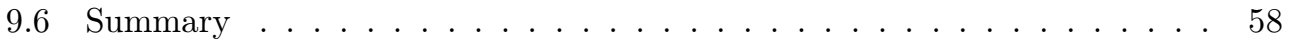

10 Study of inelastic d-d and p-d interactions for observation of neutronproton system under strong compression ${ }^{10} \quad \mathbf{6 0}$

10.1 Introduction . . . . . . . . . . . . . . . . . . 60

10.2 Search for new dibaryons at the NICA SPD facility . . . . . . . . . 61

11 Proposal for the study of lightest neutral hypernuclei

with strangeness -1 and $-2^{11} \quad 65$

11.1 Binding conditions for 3 and 4-body systems with strangeness -1 and $-2 \quad 66$

11.2 Production mechanism for ${ }_{\Lambda \Lambda}^{4} \mathrm{n}$ and advantages of double $K^{+}$productions 68

11.3 Summary . . . . . . . . . . . . . . . . . . . . . . . . . 71

12 Problems of soft $p p$ interactions ${ }^{12} \quad 72$

13 Puzzles of soft photons pp, pA and AA interactions ${ }^{13} \quad 79$

13.1 The scientific program of SP study . . . . . . . . . . . . . . . 80

13.2 The preparation to experimental SP study . . . . . . . . . . . . 81

14 Hadron formation effects in heavy ion collisions ${ }^{14} \mathbf{8 2}$

14.1 The model . . . . . . . . . . . . . . . . . . . . . . . 83

14.2 Numerical results . . . . . . . . . . . . . . . . . . . . . . . . 85

14.3 Summary and conclusions . . . . . . . . . . . . . . . 85

15 Measurement of characteristics of the processes of pair production of polarized tau leptons in the SPD experiment. ${ }^{15}$

16 On Measuring Antiproton-Production Cross Sections for Dark Matter Search ${ }^{16}$

16.1 Antiproton Production Cross Sections . . . . . . . . . . . . . . . . 96

\footnotetext{
${ }^{9}$ This section is written by V.T. Kim (kim_vt@pnpi.nrcki.ru), A.A. Shavrin (shavrin.andrey.cp@gmail.com) and A.V. Zelenov (zelenov_av@pnpi.nrcki.ru)

${ }^{10}$ This section is written by B.F. Kostenko, E-mail: bkostenko@jinr.ru

${ }^{11}$ This section is written by J.-M. Richard, Q. Wang and Q. Zhao; E-mail:zhaoq@ihep.ac.cn.

${ }^{12}$ This section is written by A. Galoyan and V. Uzhinsky.

${ }^{13}$ This section is written by E. Kokoulina (E-mail:kokoulina@jinr.ru) and V.A. Nikitin (E-mail: nikitin@jinr.ru).

${ }^{14}$ This section is written by A.B. Larionov; E-mail: larionov@theor.jinr.ru

${ }^{15}$ This section is written by A. Aleshko, E. Boos (E-mail: boos@theory.sinp.msu.ru), V. Bunichev (Email: bunichev@theory.sinp.msu.ru)

${ }^{16}$ This section is written by R. El-Kholy; E-mail: relkholy@sci.cu.edu.eg.
} 
16.2 NICA SPD Contribution . . . . . . . . . . . . . . . . . . . . . . . . . . . 98

16.3 Summary . . . . . . . . . . . . . . . . . . . . . . . 99

17 Tests of fundamental discrete symmetries at NICA facility: addendum to the spin physics programme ${ }^{17} \quad 100$

17.1 Precessing spin asymmetries in the total $p d$ cross section . . . . . . . . . . 101

17.2 PV asymmetry: expectations from Standard Model . . . . . . . . . . . . . 103

17.3 The experimental strategies . . . . . . . . . . . . . . . . . . . 104

17.4 Summary and outlook . . . . . . . . . . . . . . . . 107

${ }^{17}$ This section is presented by I.A. Koop, A.I. Milstein, N.N. Nikolaev (E-mail: nikolaev@itp.ac.ru), A.S. Popov, S.G. Salnikov, P.Yu. Shatunov,Yu.M. Shatunov. 
Tests of QCD basics in the transition region

The Standard Model (SM) of fundamental interactions formulated five decades ago as a local gauge invariant theory based on the $S U(2)_{L} \times U(1)_{Y} \times S U(3)_{c}$ spontaneously broken symmetry, was perfectly confirmed by experiments in electroweak sector. The only part of this model, Quantum Chromodynamics (QCD), connected with the colored $S U(3)_{c}$ symmetry and considered as a basis of strong interactions between quarks and gluons is still under experimental verification.

At low energies, below the $\mathrm{GeV}$ region the strong interaction is described in terms of baryons exchanging mesons in accordance with the chiral effective field theory, which is based on spontaneously broken chiral symmetry of the QCD Lagrangian [1]. Recent progress in our understanding of properties of the light nuclei and nuclear reactions achieved within this approach is outlined in Refs. [2,3]. At much higher energies and high transferred 4-momenta, perturbative Quantum Chromodynamics (pQCD) characterizes the strong force in terms of quark and gluons carrying color charge, and obeying to parton distribution functions (PDF) of hadrons and nuclei. Although these two pictures are well determined in their respective energy scales, the transition between them is not well identified. Whereas the goal of the Many Purposes Detector (MPD) NICA project is to search for phase transition of the baryon matter at high temperature and high density into the quark gluon plasma in heavy-ions collision, and on this way to study properties of the early Universe, the main aim of the Spin Physics Detector (SPD) project [4] at its first stage with lower energies is quite different and, in particular, is just connected with a search for the transition region from hadron to quark-gluon degrees of freedom in theoretical describing of collisions of free nucleons or lightest nuclei. QCD predicts that hadrons produced in exclusive processes at sufficiently high 4-momentum transfer will experience diminished final (initial) state interactions. This QCD prediction named as color transparency (CT) [5], [6] may help to identify the transition between these two alternative descriptions of strong forces after the onset of CT will be observed. Another signal for the transition region in structure of the lightest nuclei is related to onset of the predicted by pQCD dimensional scaling in reactions with these nuclei. A clear indication for transition to quark degrees of freedom in strong interactions would give a formation of multiquark states, like dibaryon resonances observed in sector of light quarks [7]. Production of heavy quarks in few-nucleon systems can be related to

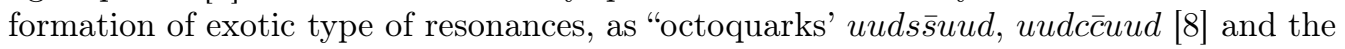
behaviour of double spin correlation $A_{N N}$ of $p p$ elastic scattering measured near the charm threshold at large angles [9] supports this assumption. On the other hand, it is important to understand how this observation is related to recently observed at $\mathrm{LHCb}$ pentaquark states $u u d c \bar{c}[10]$. The SPD NICA has all possibilities for study these and other issues of QCD. Furthermore, polarization phenomena provide an unique possibility to search for physics beyond the SM by making test of fundamental discrete symmetries of the SM related to the space $(\mathrm{P})$, time $(\mathrm{T})$ and charge $(\mathrm{C})$ inversion. One of these options is connected with double polarized proton-deuteron scattering providing a search for T-invariance (or CP-invariance under CPT-symmetry) violation.

Experiments with unpolarized colliding beams are also of importance in study of reactions at heavy quark thresholds and in search for both color transparency and scaling onset or multiquark (dibaryon) states. 


\section{The SPD setup and experimental conditions ${ }^{1}$}

The SPD experimental setup is being designed as a universal $4 \pi$ detector with advanced tracking and particle identification capabilities based on modern technologies that can operate with polarized proton and deuteron beams at a collision energy up to $27 \mathrm{GeV}$ and a luminosity up to $10^{32} \mathrm{~cm}^{-2} \mathrm{~s}^{-1}$ (proton collisions). Details of the SPD experimental setup are described in its Conceptual Design Report [4]. The silicon vertex detector will provide resolution for the vertex position on the level of below $100 \mu \mathrm{m}$ needed for reconstruction of primary and secondary vertices. The straw tube-based tracking system placed within a solenoidal magnetic field of up to $1 \mathrm{~T}$ at the detector axis should provide the transverse momentum resolution $\sigma_{p_{T}} / p_{T} \approx 2 \%$ for a particle momentum of $1 \mathrm{GeV} / c$. The time-of-flight system with a time resolution of about $60 \mathrm{ps}$ will provide $3 \sigma \pi / K$ and $K / p$ separation of up to about $1.2 \mathrm{GeV} / c$ and $2.2 \mathrm{GeV} / c$, respectively. Possible use of the aerogel-based Cherenkov detector could extend this range. Detection of photons will be provided by the sampling electromagnetic calorimeter with the energy resolution $\sim 5 \% / \sqrt{E}$. To minimize multiple scattering and photon conversion effects for photons, the detector material will be kept to a minimum throughout the internal part of the detector. The muon (range) system is planned for muon identification. It can also act as a rough hadron calorimeter. The pair of beam-beam counters and zero-degree calorimeters will be responsible for the local polarimetry and luminosity control. To minimize possible systematic effects, SPD will be equipped with a triggerless DAQ system.

It is assumed that up to $30 \%$ of the collider running time will be devoted to polarized deuteron and proton experiments from the beginning of the collider commissioning. Thus, some polarized $p p$-, $d d$ - and even $p d$ - collisions at energy range of $\sqrt{s_{N N}}=3.4 \div 10$ $\mathrm{GeV}$, could be possible already at the initial stage of the collider operation. The most accessible is polarized deuteron beam from the Nuclotron in the energy range of $1 \div 4$ $\mathrm{GeV} / \mathrm{u}$. Average luminosity of $d d$ - collisions is estimated to $8 \times 10^{27} \div 2.5 \times 10^{31} \mathrm{~cm}^{-2} \mathrm{~s}^{-1}$. Stable direction of the polarization vector is vertical. A single and double polarized collisions are possible. Transverse polarization of deuteron beam can be obtained at the specific energy point $\sim 5.6 \mathrm{GeV}$ corresponding to the spin integer resonance. The adequate intensity of polarized proton beam from the Nuclotron $\left(\geq 10^{10}\right.$ part./pulse $)$ will be reached after commissioning of the new light ion injector LILAC scheduled to 2025-2026 and the spin control system have been designed for the collider. The existing proton injected chain put limit to the beam intensity due to very low output linac energy $(5 \mathrm{MeV})$. Thus, only experiments on the beam storage and acceleration are planning for the commissioning phase. Realization of pd - mode is more complicated because HILAC and LILAC both injection chains should be involved in the process. Moreover, only single polarized collision mode is available, namely: unpolarized deuteron with polarized proton. The peak luminosity in symmetric $\mathrm{dp}$ - mode, corresponding to equal momentum of the colliding particles per nucleon, can reach of $2 \times 10^{31} \mathrm{~cm}^{-2} \mathrm{~s}^{-1}$ at stored intensity of $6 \times 10^{11}$ particles per each collider ring. Light ion collision studies at the SPD are possible also. The luminosity level can be scaled from that was specified for gold-gold collisions: $1 \times 10^{27} \mathrm{~cm}^{-2} \mathrm{~s}^{-1}$ at $\sqrt{s_{N N}}=11 \mathrm{GeV}$.

\footnotetext{
${ }^{1}$ This section is written by A.V. Guskov (E-mail: alexey.guskov@cern.ch) and A.D. Kovalenko (E-
} mail:kovalen@dubna.ru) 


\section{Elastic $p N, p d$ and $d d$ scattering ${ }^{1}$}

The spin-dependent Glauber theory is applied to calculate spin observables of $p d$ elastic scattering at 3-50 GeV/c using $p p$ amplitudes available in the literature and parametrized within the Regge formalism. The calculated vector $A_{y}^{p}, A_{y}^{d}$ and tensor $A_{x x}, A_{y y}$ analyzing powers and the spin-correlation coefficients $C_{y, y}, C_{x, x}, C_{y y, y}, C_{x x, y}$ can be measured at SPD NICA and, thus, will provide a test of the used $p N$ amplitudes. Quasi-elastic scattering $p d \rightarrow\{p p\}_{s} n$ with formation of spin-singlet $p p\left({ }^{1} S_{0}\right)$ pair at zero scattering angle is of special interest. The $d d$ elastic scattering is briefly outlined. The double polarized $p p$ and $p n$ elastic scattering at large c.m.s. scattering angle $\theta_{c m}=90^{\circ}$ is considered in the threshold of the charm production.

PACS: 25.40.Cm, 13.75.Cs, 13.88. $+\mathrm{e}$

\subsection{Spin amplitudes of $p N$ elastic scattering}

Nucleon-nucleon elastic scattering contains fundamental information on the dynamics of the $N N$ interaction and constitutes a basic process in physics of atomic nuclei and hadrons. A systematic reconstruction of spin amplitudes of $p p$ and $p n$ elastic scattering from $p N$ scattering data is provided by the SAID partial-wave analysis [11] and covers laboratory energies up to $3 \mathrm{GeV}\left(p_{l a b} \approx 3.8 \mathrm{GeV} / \mathrm{c}\right)$ for $p p$ and $1.2 \mathrm{GeV}\left(p_{l a b} \approx 1.9 \mathrm{GeV} / \mathrm{c}\right)$ for $p n$ scattering. At higher energies there is only incomplete experimental information on $p p$ scattering, whereas data for the $p n$ system are very scarce. In the literature there are several models and corresponding parametrizations for $p N$ amplitudes. Some of them are obtained in the eikonal approach for the lab momentum $6 \mathrm{GeV} / \mathrm{c}$ [12] and for LHC energies [13] and recently in [14] (see Sect. 4). At moderate transferred momenta $-t$ and large invariant mass $s$ the Regge model is expected to be valid to describe elastic $p N$ scattering. In literature there are some parametrizations for $p N$ amplitudes, obtained within the Regge phenomenology for values of $s$ above $6 \mathrm{GeV}^{2}\left(p_{l a b} \geq 2.2 \mathrm{GeV} / \mathrm{c}\right)$ [15] and for $p_{l a b}=3-50 \mathrm{GeV} / \mathrm{c}$ (corresponding to $2.77<\sqrt{s}<10 \mathrm{GeV}$ ) [16].

Assuming Lorentz-invariance and parity conservation, the elastic $N N$ scattering is described by eight independent helicity amplitudes $\phi_{i}(i=1, \ldots 8)$ determined in $[17,18]$. Under time-reversal invariance, one has $\left(\phi_{5}=\phi_{8}, \phi_{6}=\phi_{7}\right)$ six independent amplitudes, and for identical nucleons $p p$ and $n n$ the number of independent helicity amplitudes is equal to five $\left(\phi_{5}=-\phi_{6}, \phi_{7}=-\phi_{8}\right)$. Full information about the spin dependent $p N$ amplitudes can be obtained, in principle, from a complete polarization experiment, which, however, requires to measure twelve (ten) independent observables at a given collision energy for $p n$ ( $p p$ or $n n$ ) and, thus, constitutes a too complicated experimental task. Another possible way to check existing parametrizations in addition to direct measurement of spin observables of $p N$ elastic scattering is to study spin effects in proton-deuteron $(p d)$ and neutron-deuteron $(n d)$ elastic and quasi-elastic scattering. The polarized $p d$-elastic scattering is discussed below using the Glauber diffraction theory.

At large $-t$ corresponding to large scattering angles in the c.m.s. pN system $\left(\theta_{c m} \approx\right.$ $\left.90^{\circ}\right)$, where the Regge model cannot be applied, very interesting features were observed in the double spin asymmetry $A_{N N}$ in the elastic pp scattering at laboratory momenta

\footnotetext{
${ }^{1}$ This section is written by Yu.N. Uzikov; E-mail:uzikov@jinr.ru
} 


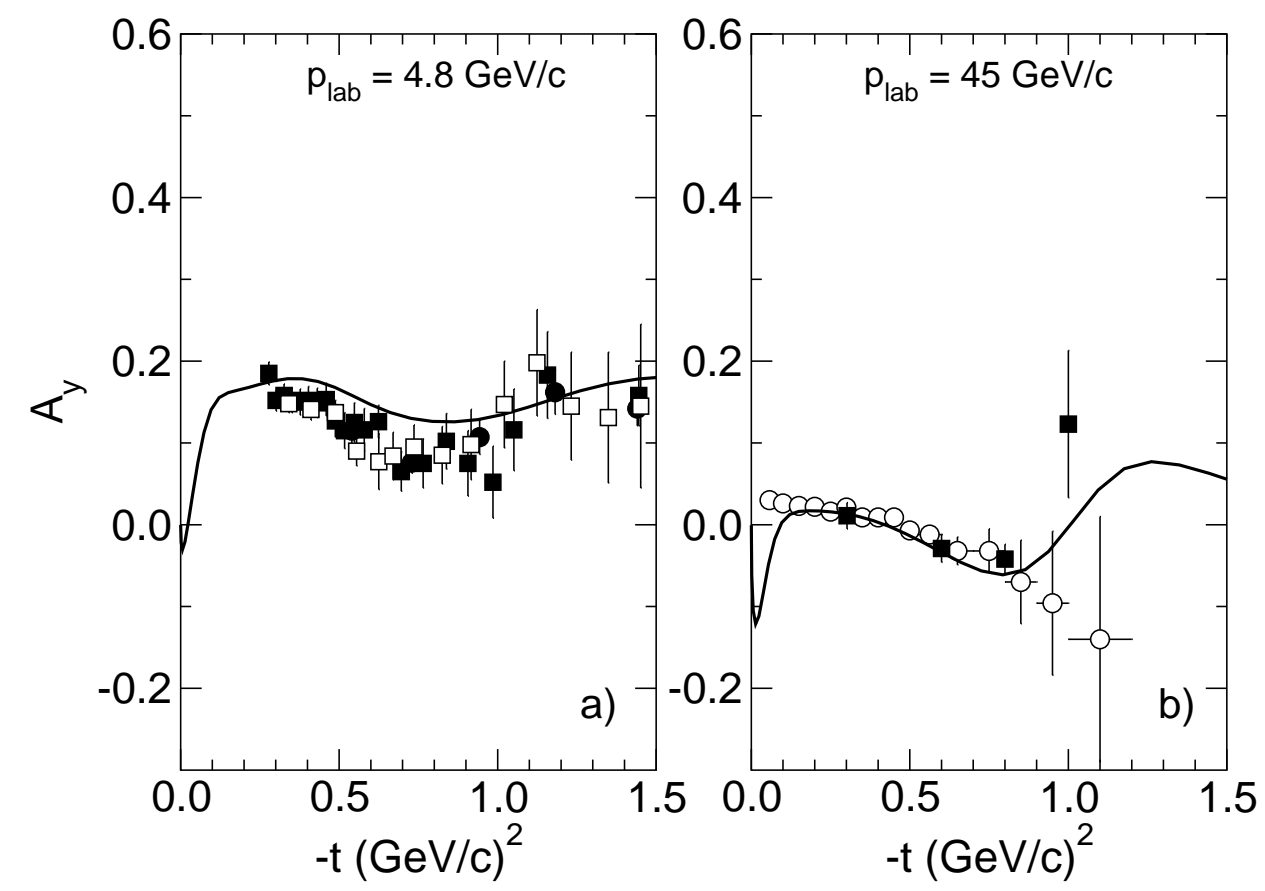

Fig. 1. Analyzing power for $p p$ elastic scattering as a function of the four-momentum transfer $-t$ at $4.8 \mathrm{GeV} / \mathrm{c}$ (left) and $45 \mathrm{GeV} / \mathrm{c}$ (right). The results of calculations [23] based on the Regge model parameterizations from [16] are shown by the solid line (see details in Ref. [23]). Left: Data are taken from Refs. [24] (filled squares: $4.4 \mathrm{GeV} / \mathrm{c}$; open squares: $5.15 \mathrm{GeV} / \mathrm{c}$ ), and [25] (circles). Right: Data are taken from Refs. [26] (squares) and [27] (circles).

$p_{l a b}=5-10 \mathrm{GeV} / \mathrm{c}$. Commonly accepted explanation of those features is absent in literature. In section 2.5 we give a short review of existing models based on usage of the pQCD amplitudes and non-perturbative exotic multiquark resonances contribution.

\subsection{Polarized $p d$ elastic diffraction scattering within the Glauber model}

As was noted above, a possible way to check existing parametrizations of $p N$ elastic amplitudes is to study spin effects in proton-deuteron $(p d)$ and deuteron-deuteron $(d d)$ elastic and quasi-elastic scattering. At high energies and small four-momentum transfer $t$, $p d$ scattering can be described by the Glauber diffraction theory of multistep scattering, which involves as input on-shell $p N$ elastic scattering amplitudes. Applications of this theory with spin-dependent effects included [19] indicate a good agreement with the $p d$ scattering data at energies about $1 \mathrm{GeV}$ if the SAID data on $p N$ scattering amplitudes are used as input of the calculations [20-22].

The spin-dependent Glauber theory $[19,20]$ is applied recently [23] to calculate spin observables of $p d$ elastic scattering at 3-50 GeV/c utilizing the $p p$ elastic scattering amplitudes $f_{p p}$ established and parametrized in Ref. [16] within the Regge formalism. The Regge approach allows one to construct $p n$ (and $\bar{p} N$ ) amplitudes together with the $p p$ 
amplitudes. This feature allows one to perform a test of broad set of $p N$ amplitudes and applicability of the Regge model itself to $p N$ elastic scattering. However, in view of the scarce experimental information about the spin-dependent $p n$ amplitudes and taking into account that the spin-independent parts of the $p p$ and $p n$ amplitudes at high energies are approximately the same, it was assumed in [23] as a first approximation, that $f_{p n}=f_{p p}$. The amplitudes of $p N$ elastic scattering are written as [19]

$$
\begin{array}{r}
M_{N}\left(\mathbf{p}, \mathbf{q} ; \boldsymbol{\sigma}, \boldsymbol{\sigma}_{N}\right)=A_{N}+C_{N} \boldsymbol{\sigma} \hat{n}+C_{N}^{\prime} \boldsymbol{\sigma}_{N} \hat{n}+B_{N}(\boldsymbol{\sigma} \hat{\mathbf{k}})\left(\boldsymbol{\sigma}_{N} \hat{\mathbf{k}}\right)+ \\
+\left(G_{N}+H_{N}\right)(\boldsymbol{\sigma} \hat{\mathbf{q}})\left(\boldsymbol{\sigma}_{N} \hat{\mathbf{q}}\right)+\left(G_{N}-H_{N}\right)(\boldsymbol{\sigma} \hat{\mathbf{n}})\left(\boldsymbol{\sigma}_{N} \hat{\mathbf{n}}\right)
\end{array}
$$

where the complex numbers $A_{N}, C_{N}, C_{N}^{\prime}, B_{N}, G_{N}, H_{N}$ were fixed from the amplitudes of the SAID analysis [11] and parametrized by a sum of Gaussians. For the double scattering term in $p d$ scattering the unit vectors $\hat{\mathbf{k}}, \hat{\mathbf{q}}, \hat{\mathbf{n}}$ are defined separately for each individual $N N$ collision. Numerical values for the parameters of the Gaussians are obtained by fitting to the helicity amplitudes from Ref. [16]. Those for $p_{l a b}=45 \mathrm{GeV} / \mathrm{c}$ are given in Ref. [23]. The differential cross section of $p p$ elastic scattering and the vector analyzing power $A_{y}$ are reproduced with these parameterizations on the same level of accuracy as in Ref. [16], in the interval of transferred four momentum $-t<1.5(\mathrm{GeV} / \mathrm{c})^{2}$. An example of calculations of $A_{y}$ at $p_{l a b}=4.8 \mathrm{GeV} / \mathrm{c}$ and $45 \mathrm{GeV} / \mathrm{c}$ is shown in Fig. 1 .

The spin observables $A_{y}, A_{i j}$, and $C_{i j, k}$ considered in the work [23] are defined in the notation of Ref. [28] as following

$$
\begin{array}{r}
A_{y}^{d}=\operatorname{Tr} M S_{y} M^{+} / \operatorname{Tr} M M^{+}, A_{y}^{p}=\operatorname{Tr} M \sigma_{y} M^{+} / \operatorname{Tr} M M^{+} \\
A_{y y}=\operatorname{Tr} M \mathcal{P}_{y y} M^{+} / \operatorname{Tr} M M^{+}, A_{x x}=\operatorname{Tr} M \mathcal{P}_{y y} M^{+} / \operatorname{Tr} M M^{+} \\
C_{y, y}=\operatorname{Tr} M S_{y} \sigma_{y} M^{+} / \operatorname{Tr} M M^{+}, C_{x, x}=\operatorname{Tr} M S_{y} \sigma_{y} M^{+} / \operatorname{Tr} M M^{+}, \\
C_{x x, y}=\operatorname{Tr} M \mathcal{P}_{x x} \sigma_{y} M^{+} / \operatorname{Tr} M M^{+}, C_{y y, y}=\operatorname{Tr} M \mathcal{P}_{y y} \sigma_{y} M^{+} / \operatorname{Tr} M M^{+},
\end{array}
$$

where $\mathcal{P}_{i j}=\frac{3}{2}\left(S_{i} S_{j}+S_{j} S_{i}\right)-2 \delta_{i j}$ and $S_{j}(j=x, y, z)$ are Cartesian components of the spin operator for the system with $S=1$, the transition operator $M$ depends on the momentum of the initial $(\mathbf{p})$ and final $\left(\mathbf{p}^{\prime}\right)$ proton and contains the Pauli spin matrices $\boldsymbol{\sigma}=\left(\sigma_{x}, \sigma_{y}, \sigma_{z}\right)$. We use the Madison reference frame with the axis OZ $\|\mathbf{p}, \mathrm{OY}\|\left[\mathbf{p} \times \mathbf{p}^{\prime}\right]$ and OX choosen in such a way to provide a right-handed coordinate system.

The unpolarized differential cross section, vector $\left(A_{y}^{p}, A_{y}^{d}\right)$ and tensor $\left(A_{x x}, A_{y y}\right)$ analyzing powers and some spin correlation parameters $\left(C_{x, x}, C_{y, y}, C_{x x, y}, C_{y y, y}\right)^{1}$ of $p d$ elastic scattering were calculated at $p_{l}=4.85 \mathrm{GeV} / \mathrm{c}$ and $45 \mathrm{GeV} / \mathrm{c}$ at $0<-t<2$ $\mathrm{GeV}^{2}$ using $p N$ amplitudes from [16]. The results obtained for $A_{y}^{p}, A_{y}^{d}, C_{x x, y}$ and $C_{y y, y}$ are shown in Fig. 2. As shown in Ref. [23] available data on pd-elastic differential cross section in forward hemisphere are well described by this model. Most sensitive to the spin-dependent $p N$ amplitudes are vector analyzing powers $A_{y}$ and spin correlation parameters $C_{x, x}$ and $C_{y, y}$. So, even measurement of the ratio $A_{y}^{d} / A_{y}^{p}$ at low $t$ gives valuable information on the transverse spin-spin term in $\mathrm{NN}$-amplitudes [29]. In contrast, the tenzor analyzing powers $A_{x x}$ and $A_{x x}$ are very weakly sensitive to those

\footnotetext{
${ }^{1}$ We use here notations of Ref. [28]
} 

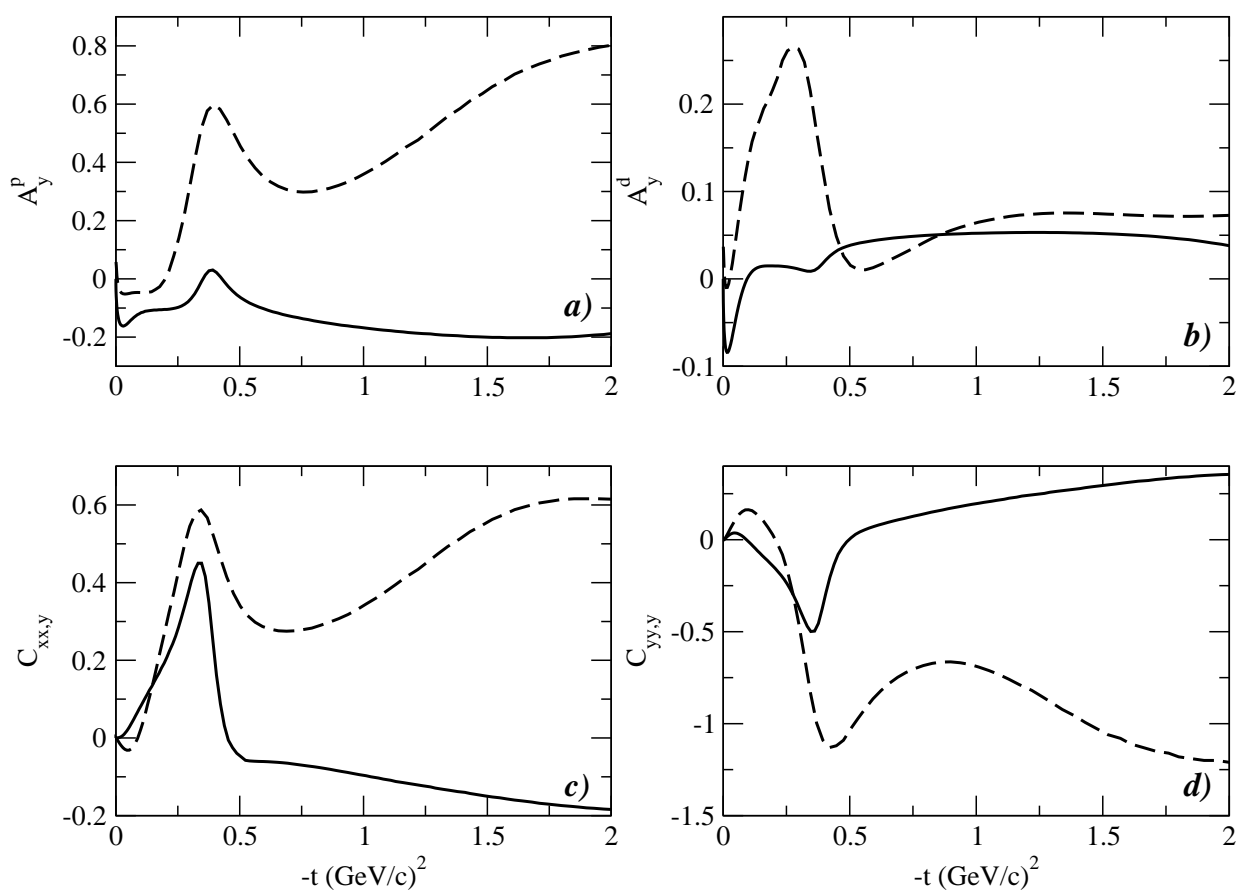

Fig. 2. Results for spin-dependent $p d$ observables. Predictions from Ref. [23] for $p_{\text {lab }}=4.8$ $\mathrm{GeV} / \mathrm{c}$ are shown by dashed lines while those at $45 \mathrm{GeV} / \mathrm{c}$ correspond to the solid lines.

amplitudes and weakly changed with increasing energy. The calculated in [23] polarization observables can be measured at SPD NICA that will provide a test of the used $p N$ amplitudes. The corresponding differential cross section is rather large in the considered region $p_{l a b}=3-50 \mathrm{GeV} / \mathrm{c}$ and $|t|=0-2 \mathrm{GeV}^{2}$ being $d \sigma / d t>0.1 \mathrm{mb} / \mathrm{GeV}^{2}$. Expected counting rate $N$ at $p_{l a b}=50 \mathrm{GeV} / \mathrm{c}\left(q_{p p}^{c m}=5 \mathrm{GeV} / \mathrm{c}\right)$ for the luminosity $L=5 \times 10^{30} \mathrm{~cm}^{-2} \mathrm{~s}^{-1}$ and for the solid angle $\Delta \Omega=0.03$ is $N \geq 10^{2} \mathrm{~s}^{-1}$.

The $p N$ helicity amplitudes $\phi_{5}$ and $\phi_{1}+\phi_{3}$, which can be tested in the above described procedure are necessary in search of time-reversal invariance effects in double-polarized $p d$ scattering [30,31]. Data of the spin-correlation parameters of $p p$ elastic scattering being analyzed in the framework of the eikonal model [13] will allow one to obtain space structure of the spin-dependent hadron forces [32].

\subsection{Quasielastic pd-scattering $p+d \rightarrow\{p p\}\left({ }^{1} S_{0}\right)+n$}

Spin structure of the amplitude of the reaction of quasielastic $p d$ scattering with formation of the $p p$ pair at small excitation energy $\leq 3 \mathrm{MeV}$

$$
p+d \rightarrow\{p p\}\left({ }^{1} S_{0}\right)+n
$$

is of special interest. In this reaction the final $p p$ pair is in the ${ }^{1} S_{0}$ state of the internal motion, therefore the number of of independent transition matrix elements is diminished 
to six instead of twelve for the elastic $p d$ - scattering. Since the angular momentum of the $p p\left({ }^{1} S_{0}\right)$ pair is zero, in collinear kinematics the transition matrix element of this reaction is completely described by two independent amplitudes $\mathcal{A}$ and $\mathcal{B}$ as following

$$
\mathcal{F}=\mathcal{A}(\mathbf{e} \cdot \mathbf{k})(\boldsymbol{\sigma} \cdot \mathbf{k})+\mathcal{B} \mathbf{e} \cdot \boldsymbol{\sigma}
$$

where $\mathbf{k}$ is unit vector directed along the beam, $\mathbf{e}$ is the deuteron polarization vector and $\boldsymbol{\sigma}$ is the Pauli matrix. The modules of these amplitudes and cosine of the relative phase $\varphi_{A B}$ can be determined by measurement of unpolarized cross section of the reaction $d \sigma_{0}$ and tenzor analyzing powers $T_{20}=A_{z z} / \sqrt{2}$ and $A_{y y}$. In order to measure the sine of the relative phase $\varphi_{A B}$ one has to measure only the sign of the spin-correlation coefficient $C_{x z, y}$.

Within the approximation of the $p n$ - single scattering the theoretical analysis of this reaction becomes more simple. In this case the $\mathcal{A}$ and $\mathcal{B}$ amplitudes of the reaction (3) are expressed via the spin amplitudes of the charge exchange reaction

$$
p+n \rightarrow n+p
$$

The transition matrix element of reaction (5) at zero scattering angle can be written as

$$
f_{12}^{\text {collin }}=\alpha+\beta\left(\boldsymbol{\sigma}_{1} \cdot \boldsymbol{\sigma}_{2}\right)+(\varepsilon-\beta)\left(\boldsymbol{\sigma}_{1} \cdot \mathbf{k}\right)\left(\boldsymbol{\sigma}_{2} \cdot \mathbf{k}\right)
$$

where $\boldsymbol{\sigma}_{1}\left(\boldsymbol{\sigma}_{2}\right)$ the Pauli matrix acting on the spin state of the first (second) nucleon.

We can show that measurement of $d \sigma_{0}$ and $T_{20}$ provides the modules of $|\varepsilon|$ and $|\beta|$ whereas the cosine of the relative phase ( or $\operatorname{Re} \varepsilon \beta^{*}$ ) is determined by the spin correlation parameters $C_{x, x}=C_{y, y}$. In order to measure the sine of this phase ( $\left.\operatorname{Im} \beta \varepsilon^{*}\right)$ one has to measure the sign of $C_{x z, y}\left(=-C_{y z, x}\right)$. Therefore, measurement of $d \sigma_{0}, T_{20}, C_{y, y}$ and the sign of $C_{x z, y}$ at zero scattering angle completely determines the spin amplitudes $\varepsilon$ and $\beta$.

\subsection{Elastic $d d$ scattering}

Spin observables of the $d d$ - elastic scattering in forward hemisphere also can be used to test spin-dependent amplitudes of $p N$ elastic scattering since the Glauber model can be used for description of these observables. Unpolarized differential cross section of the $d d$ elastic scattering in forward hemisphere measured at energies $\sqrt{s}=53-63 \mathrm{GeV}$ [33] was well described by the modified Glauber theory including Gribov inelastic corrections. At lower energies corresponding to the SPD NICA region, one may expect that inelastic corrections are not important, that can be checked by direct calculation of unpolarized cross section and subsequent comparison with the data. In this calculations the above considered spin dependent amplitudes of the $p d$ elastic scattering [23] can be used as input for the Glauber calculations of the $d d$ scattering.

At large scattering angles $\theta_{c m} \sim 90^{\circ}$ the $p d \rightarrow p d$ and $d d \rightarrow d d$ processes are sensitive to the short-range (six-quark) structure of the deuteron. Therefore, measurement of any observables of these processes at large $\theta_{c m}$ will be important to search for non-nucleonic degrees of freedom of the deuteron. 


\subsection{Double polarized large angle $p N$ elastic scattering}

The $p p$ and $p n$ elastic scattering at high energy $\sqrt{s}=5-7 \mathrm{GeV}$ and large transferred momentum $-t=5-10 \mathrm{GeV}^{2}$ is powered by short-range properties of NN-interaction corresponding to small separation between nucleons $r_{N N} \sim \hbar / \sqrt{-t} \leq 0.1 \mathrm{fm}$. There are three following aspects of QCD dynamics in these processes. ( $i$ ) First, the differential cross section $d \sigma^{p p} / d t\left(s, \theta_{c m}\right)$ at fixed angle $\theta_{c m} \sim 90^{\circ}$ on the whole follows to the pQCD constituent counting rules $d \sigma^{p p} / d t\left(s, \theta_{c m}\right) \sim s^{-10}$ [34-37]. However, a clear deviation from this prediction in form of oscillations with increasing energy is observed in the region $s=10 \div 40 \mathrm{GeV}^{2}$ [34-37]. The irregularity in the energy dependence is on the level of $\sim 50 \%$ in the region, where magnitude of the elastic pp- cross section falls down by 8 orders of magnitude. (ii) Second, anomalous polarization asymmetries were observed in hard pN-scattering at $p_{l a b}=11.75 \mathrm{GeV} / \mathrm{c}[9,38,39]$. Elastic $p p$-cross section with spins of protons parallel and normal to the scattering plane is almost four time larger than the cross section with antiparallel spins. The challenge is that in order to generate such large polarization effect, one needs to have large contribution from double spin-flip helicity amplitude $\phi_{2}$ or negligible contribution from helicity conserving $\phi_{1}$ amplitude. However, in pQCD, in contrast, $\phi_{2}$ is the most suppressed and the $\phi_{1}$ is largest [40]. Predicted within the pQCD (quark-interchange model) double spin asymmetry $A_{N N}$ does not depend on energy [41], [42], whereas the measured asymmetry demonstrates "oscillating" energy dependence. (iii) The third QCD aspect of hard NN scattering is related to the Color Transparency phenomenon (CT), that is a reduction of the absorption in the nuclear medium of hard produced hadrons, both mesons and baryons [5], [6]. Being in point like configurations, which are dictated by mechanism of high momentum transfer, the initial and final hadrons participating in hard process have small color dipole momenta and, therefore, small interaction cross section with nuclear medium. These expectations resulted in huge theoretical and experimental activities in 90's. While the CT effect is observed for the hard production of the $q \bar{q}$ systems, the similar effect for $q q q$ is elusive. The data $[43,44]$ on the reaction $p+A \rightarrow p p+X$ on the ${ }^{12} \mathrm{C}$ and ${ }^{27} \mathrm{Al}$ show again an "oscillatory" effect, i.e. the transparency increases with increasing momentum up to $p_{l b}=9$ $\mathrm{GeV} / \mathrm{c}$, and then decreases below the Glauber calculation predictions at $14 \mathrm{GeV} / \mathrm{c}$. An attempt to connect all three above aspects together into one approach was undertaken in Ref. [40]. However, recent measurement of the cross section of the reaction ${ }^{12} \mathrm{C}(\mathrm{e}, \mathrm{ep}) \mathrm{X}$ at $Q^{2}=8-14(\mathrm{GeV} / \mathrm{c})^{2}[45]$ shows no CT effect and this fact raises new questions to the analysis made in [40]. On the other hand, according to [8], the observed large variations in spin correlations of $p p$-elastic scattering are consistent with formation in the s-channel

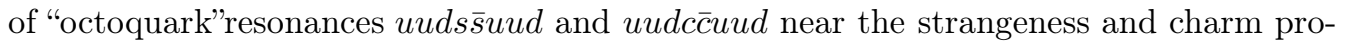
duction thresholds, respectively. The variations with increasing energy are explained as a result of interference of the pQCD background amplitude with nonperturbative resonant amplitudes. Furthermore, the model [8] provides a description of the oscillations in the unpolarized differential $p p$ - elastic cross section.One should mentioned, however, that another explanation of the oscillation effect in the $d \sigma^{p p} / d t\left(s, \theta_{c m}\right)$ was suggested in Ref. [46].

The considered questions about new types of charm-based resonances [47] became especially interesting after observation enhancement effects in the decay $\Lambda_{b}^{0} \rightarrow J / \Psi p K^{-}$ interpreted as pentaquark states $u u d c \bar{c}$ [10] (see also Ref. [47]). More insight into this 
issue can be get from the data on large angle $p n$ elastic scattering. Different spin-isospin structure of the transition matrix elements for the near threshold $J / \Psi$ production in $p n$ and $p p$ collisions [48] means that spin observables in $p n$ - elastic scattering can give a valuable independent information on the considered dynamics. Data on these observables are almost absent in the considered energy region. A task to get such data in the energy interval $\sqrt{s_{N N}} \approx 3 \div 5 \mathrm{GeV}$ from the $\vec{p} \vec{d} \rightarrow p n p$ and $\vec{d} \vec{d} \rightarrow$ pnpn reactions is accessible for the SPD NCA.

\subsection{Summary}

To conclusion, nucleon-nucleon elastic scattering is a basic process in the physics of atomic nuclei and the interaction of hadrons with nuclei. Existing models and corresponding parametrizations of $p p$ amplitudes in the region of small transferred momenta can be effectively tested by a measurement of spin observables for $p d$ and $d d$ elastic scattering and a subsequent comparison of the results with corresponding Glauber calculations. The spin observables of $p d$ elastic scattering studied and evaluated in [23] are found to be not too small and, thus, could be measured at the future SPD NICA facility. As extension of this study, the quasi-elastic processes with formation of the spin-singlet final $N N$-pair at small excitation energy $<3 \mathrm{MeV}$ in the ${ }^{1} S_{0}$ state of internal motion, $p d \rightarrow n\{p p\}_{s}$ and $p d \rightarrow p\{p n\}_{s}$ can be also investigated. 


\section{Studying periphery of the nucleon in diffractive $p p$ scattering ${ }^{1}$}

Motivation is outlined for a precise study of high-energy diffractive scattering of protons at $|t| \lesssim 1 \mathrm{GeV}^{2}$ in the experiment SPD. Small oscillations in the $t$-dependence of the differential cross section at low and medium $t$, observed in earlier experiments at Protvino, ISR, Fermilab and now also at LHC, are probably related with the proton's structure at impact parameters exceeding the size of the proton's quark core and thus indicate involvement of meson periphery of the nucleon to diffractive scattering. The experiment SPD can provide new precise data on small-angle elastic $p p$-scattering for exploring this phenomenon.

1. Scattering of high-energy hadrons at low $t$ is usually described by a simple phenomenological dependence $d \sigma / d t=A e^{B t}$ (not applicable in the Coulomb region, $|t| \lesssim 0.01 \mathrm{GeV}^{2}$, and at $\left.|t| \gtrsim 0.4 \mathrm{GeV}^{2}\right)$. In the impact parameter representation, such a dependence corresponds to a gaussian profile function $\Gamma(b) \sim \exp \left(-b^{2} / 2 B\right)$ with the average transverse size $\left\langle b^{2}\right\rangle^{1 / 2}=B^{1 / 2} \sim 0.6 \mathrm{fm}$ when $B \sim 10 \mathrm{GeV}^{-2}$. This size corresponds well to the quark core size of the nucleon, $r_{q} \sim 0.4-0.5 \mathrm{fm}$, where the bulk of the nucleon mass (and the energy and momentum) is concentrated.

On the other hand, part of the nucleon components is clearly located at larger distances, pion cloud being the most evident example. The first evidence of the pion cloud effect in the diffractive scattering, including rapid variation of the effective slope $B$ at $|t| \sim 0.1 \mathrm{GeV}^{2} \approx 4 m_{\pi}^{2}$, have been found in ISR measurements (a comprehensive review of the ISR data can be found in [49]).

First explanations of this, presumably pion cloud effect, were provided by Anselm and Gribov [50] (see also [51,52]). Soon then a dedicated experiment was conducted in Protvino [53] in order to test the ISR results. However, beyond confirming findings of ISR, one more oscillation in the differential cross section at $|t| \sim 0.5 \mathrm{GeV}^{2}$ was found. Being located at higher $t$, it might be related with somewhat heavier mesons around the proton (but not as heavy as vector mesons that are too heavy).

Actually, S.P. Denisov et al. recently suggested to continue exploring $p p$ elastic scattering in that kinematical region at Protvino [54], and the current proposal of doing similar experiment at SPD was directly motivated by the Denisov's ideas.

2. It is essential that the Protvino experiment is not the only work indicating an oscillation at $|t| \sim 0.5 \mathrm{GeV}^{2}$ in the fine structure of the $p p$ diffraction cone. In Fig. 3 the most precise data of three experiments - from Protvino [53] (at the proton beam momentum $p=60 \mathrm{GeV} / c$ ), ISR [49] (at the total energy $\sqrt{s}=52.8 \mathrm{GeV}$ ), and Fermilab [55] (at $p=200 \mathrm{GeV} / c$ ), see also a comprehensive compilation and parameterization of world data in [56] - are compared to exponential form $F(t)=A e^{B t+C t^{2}}$ beyond the Coulomb region of tiny $|t|$ and the region of $|t| \lesssim 0.1 \mathrm{GeV}^{2}$ where effects of the pion cloud contribute. One has to notice that ISR and FNAL data do not fully cover the region of $t$ with suspected oscillations and do not have sufficient accuracy there. Therefore, further experimental studies in that region are well justified.

${ }^{1}$ This section is written by V.A. Baskov, O.D. Dalkarov, A.I. L'vov (E-mail: lvov@x4u.lebedev.ru) and V.V. Polyanskiy 
IHEP: $(\mathbf{d} \sigma / \mathbf{d t}) / \mathbf{F}$

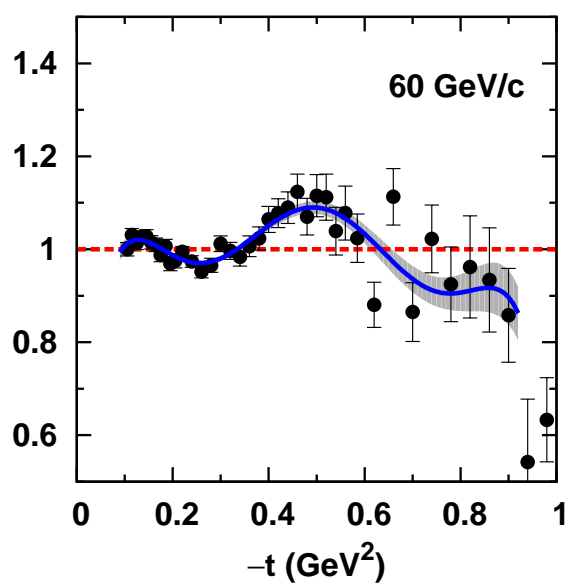

ISR: $(\mathbf{d} \sigma / d t) / F$

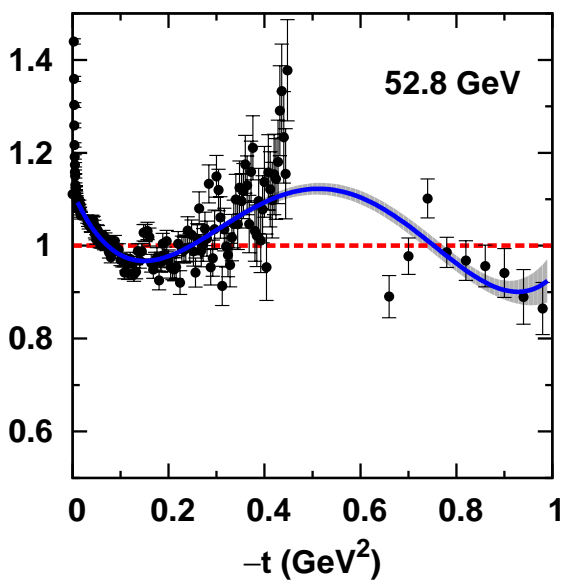

FNAL: (d $\sigma / d t) / F$

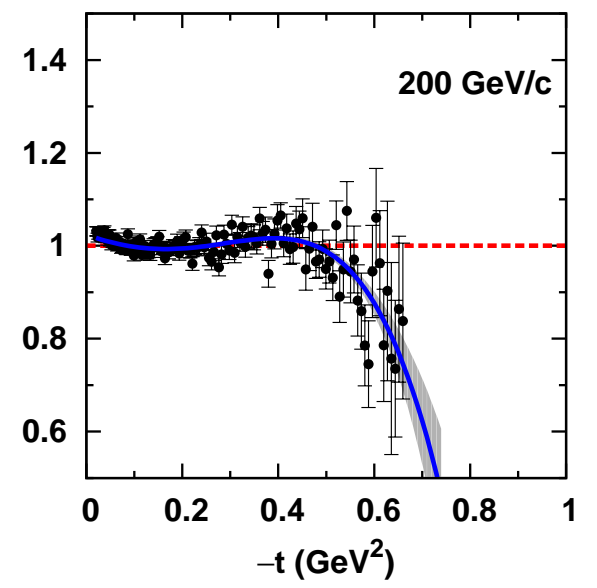

Fig. 3. Deviations of the $p p$ differential cross section from smooth dependences $F(t)=A e^{B t+C t^{2}}$. Data are from Protvino, ISR and FNAL, see in the text. Solid line is a polynomial smoothing of shown ratios. Strips show statistical errors in the polynomial.

In principle. information on the smoothed ratios $R(t)=(d \sigma / d t) / F(t)$ could be used in order to estimate the $p p$ scattering amplitude $f(s, t)$ and to find then, through a Fourier-Bessel transformation, a profile function $\Gamma(b)$ of the impact parameter $b$ [57]. A peak in $f(s, t)$ at $|t| \sim 0.5 \mathrm{GeV}^{2}$ corresponds to a peak in the profile function $\Gamma(b)$ at large distances $b \sim 7.0 / \sqrt{|t|} \sim 2 \mathrm{fm}$ (here 7.0 is the second maximum of the Bessel function $J_{0}(x)$ ), However, a straightforward calculation of $\Gamma(b)$ in this way does not give reliable results in the region where $\Gamma(b)$ becomes very small and sensitive to assumed phase of the amplitude used, its spin structure, behavior at higher $|t|$, etc. Actually, 
more sophisticated and indirect approaches are to be used in order to analyze data on the described oscillation - see, for example, [58-60].

3. In order to cover the region of interest, $|t| \sim 0.1-0.8 \mathrm{GeV}^{2}$, the experimental setup must detect protons (in coincidence) scattered at angles $\theta \sim 3-10^{\circ}$ what needs detectors placed at distances $R \sim 4-15 \mathrm{~cm}$ from the beam. Accuracy of determination of the momentum transfer squared $t$ in individual events of elastic $p p$-scattering must be better than $\Delta t \sim 0.01-0.02 \mathrm{GeV}^{2}$, and this can be achieved with planned tracker endcap detectors and with angular spread of colliding protons determined by beam emittance and beta-function at IP.

Additional measurements of $d \sigma / d t$ and/or polarization observables at higher $t$ are also desirable; they do not require high accuracy in determination of $t$ [61].

Vertex detector, tracker system and software for track reconstruction in SPD are sufficient for identification and recording $p p$ elastic events at energies $\sqrt{s} \lesssim 15 \mathrm{GeV}$. For higher energies and smaller angles, when scattered protons fly very close to the beam pipe, installing fast detectors close to the pipe, with the time resolution $\Delta T \lesssim 50 \mathrm{ps,}$ for determination of hitting times of forward-flying protons (perhaps, using the so-called PID system) would make it possible to study the discussed anomaly at the highest SPD energies. 


\section{Hadron structure and spin effects in elastic hadron scattering at NICA energies ${ }^{1}$}

The spin effects in the elastic proton-proton scattering are analysed at NICA energies. It is shown the importance the investigation of the region of the diffraction minimum in the differential cross sections. Some possible estimation of spin effects are given for the different NICA energies in the framework of the new high energy generelazed structure (HEGS) model.

PACS: 13.40.Gp, 14.20.Dh, 12.38.Lg

One of the most important tasks of modern physics is the research into the basic properties of hadron interaction. The dynamics of strong interactions finds its most complete representation in elastic scattering. It is just this process that allows the verification of the results obtained from the main principles of quantum field theory: the concept of the scattering amplitude as a unified analytic function of its kinematic variables connecting different reaction channels were introduced in the dispersion theory by N.N. Bogoliubov [62]. Now many questions of hadron interactions are connected with modern problems of astrophysics such as unitarity and the optical theorem [63], and problems of baryon-antibaryon symmetry and CP-invariance violation [30] The main domain of elastic scattering is small angles. Only in this region of interactions can we measure the basic properties that define the hadron structure. Their values are connected, on the one hand, with the large-scale structure of hadrons and, on the other hand, with the first principles that lead to the theorems on the behavior of scattering amplitudes at asymptotic energies [64,65].

Modern studies of elastic scattering of high energy protons lead to several unexpected results reviewed, e.g., in [66,67]. Spin amplitudes of the elastic $N N$ scattering constitute a spin picture of the nucleon. Without knowledge of the spin $N N$-amplitudes it is not possible to understand spin observable of nucleon scattering off nuclei. In the modern picture, the structure of hadrons is determined by Generalized Distribution functions (GPDs), which include the corresponding parton distributions (PDFs). The sum rule [68] allow to obtain the elastic form factor (electromagnetic and gravitomagnetic) through the first and second integration moments of GPDs. It leads to remarkable properties of GPDs - some corresponding to inelastic and elastic scattering of hadrons. Now some different models examining the nonperturbative instanton contribution lead to sufficiently large spin effects at superhigh energies $[69,70]$ The research of such spin effects will be a crucial stone for different models and will help us to understand the interaction and structure of particles, especially at large distances. There are large programs of researching spin effects at different accelerators. Especially, we should like to note the programs at NICA, where the polarization of both the collider beams will be constructed. So it is very important to obtain reliable predictions for the spin asymmetries at these energies. In this paper, we extend the model predictions to spin asymmetries in the NICA energy domain.

\footnotetext{
${ }^{1}$ This section is written by O.V. Selyugin; E-mail: selugin@theor.jinr.ru
} 
The NICA SPD detector bounded a very small momentum transfer. If in the first steps the angles start from $16 \mathrm{mrad}$, then the minimum momentum transfer, which can be measured is more than $-0.01 \mathrm{GeV}^{2}$. Hence it is needed to exclude the Coulomb-nuclear interference region, where the real part of the spin-non-flip amplitude can be determined, We should move our researches on the region of the diffraction minimum, where the imaginary part of the spin-non-flip amplitude changes its sign. Note that in some models the absence of the second diffraction minimum is explained by the contribution in the differential cross section of the spin-flip amplitude [71] The interference of the hadronic and electromagnetic amplitudes may give an important contribution not only at very small transfer momenta but also in the range of the diffraction minimum [72]. However, for that one should know the phase of the interference of the Columbic and hadronic amplitude at sufficiently large transfer momenta too.

Using the existing model of nucleon elastic scattering at high energies $\sqrt{s}>9 \mathrm{GeV}$ - $14 \mathrm{TeV}$ [58,73], which involves minimum of free parameters, we are going to develop its extended version aimed to describe all available data on cross sections and spincorrelation parameters at lower energies down to the SPD NICA region. The model will be based on the usage of known information on generalized parton distributions in the nucleon, electro-magnetic and gravitomagnetic form factors of the nucleon taking into account analyticity and unitarity requirements and providing compatibility with the high energy limit, where the pomeron exchange dominates.

\subsection{HEGS model and spin effects in the dip region of momentum transfer}

The differential cross sections of nucleon-nucleon elastic scattering can be written as a sum of different helicity amplitudes:

$$
\begin{gathered}
\frac{d \sigma}{d t}=\frac{2 \pi}{s^{2}}\left(\left|\Phi_{1}\right|^{2}+\left|\Phi_{2}\right|^{2}+\left|\Phi_{3}\right|^{2}+\left|\Phi_{4}\right|^{2}+4\left|\Phi_{5}\right|^{2}\right. \\
A_{N} \frac{d \sigma}{d t}=-\frac{4 \pi}{s^{2}}\left[\operatorname{Im}\left(\Phi_{1}(s, t)+\Phi_{2}(s, t)+\Phi_{3}(s, t)-\Phi_{4}\right)(s, t) \Phi_{5}^{*}(s, t)\right]
\end{gathered}
$$

and

$$
A_{N N} \frac{d \sigma}{d t}=\frac{4 \pi}{s^{2}}\left[\operatorname{Re}\left(\Phi_{1}(s, t) \Phi_{2}^{*}(s, t)-\Phi_{3}(s, t) \Phi_{4}^{*}\right)(s, t)+\left|\Phi_{5}(s, t)\right|^{2}\right]
$$

The HEGS model $[58,73]$ takes into account all five spiral electromagnetic amplitudes. The electromagnetic amplitude can be calculated in the framework of QED. In the high energy approximation, it can be obtained [74] for the spin-non-flip amplitudes:

$$
F_{1}^{e m}(t)=\alpha f_{1}^{2}(t) \frac{s-2 m^{2}}{t} ; \quad F_{3}^{e m}(t)=F_{1}^{e m} ;
$$

and for the spin-flip amplitudes: with the electromagnetic and hadronic interactions included, every amplitude $\phi_{i}(s, t)$ can be described as

$$
\phi_{i}(s, t)=F_{i}^{e m} \exp (i \alpha \varphi(s, t))+F_{i}^{h}(s, t),
$$


where $\varphi(s, t)=\varphi_{C}(t)-\varphi_{C h}(s, t)$, and $\varphi_{C}(t)$ will be calculated in the second Born approximation in order to allow the evaluation of the Coulomb-hadron interference term $\varphi_{C h}(s, t)$. The quantity $\varphi(s, t)$ has been calculated at large momentum transfer including the region of the diffaction minimum $[72,75,76]$ and references therein.

Let us define the hadronic spin-non-flip amplitudes as

$$
F_{\mathrm{nf}}^{h}(s, t)=\left[\Phi_{1}(s, t)+\Phi_{3}(s, t)\right] / 2
$$

The model is based on the idea that at high energies a hadron interaction in the nonperturbative regime is determined by the reggenized-gluon exchange. The cross-even part of this amplitude can have two non-perturbative parts, possible standard pomeron - $\left(P_{2 n p}\right)$ and cross-even part of the 3 -non-perturbative gluons $\left(P_{3 n p}\right)$. The interaction of these two objects is proportional to two different form factors of the hadron. This is the main assumption of the model. The second important assumption is that we choose the slope of the second term four times smaller than the slope of the first term, by analogy with the two pomeron cuts. Both terms have the same intercept.

The form factors are determined by the Generalized parton distributions of the hadron (GPDs). The first form factor corresponding to the first momentum of GPDs is the standard electromagnetic form factor $-G(t)$. The second form factor is determined by the second momentum of GPDs $-A(t)$. The parameters and $t$-dependence of GPDs are determined by the standard parton distribution functions, so by experimental data on deep inelastic scattering and by experimental data for the electromagnetic form factors (see [13]). The calculations of the form factors were carried out in [77]. The final elastic hadron scattering amplitude is obtained after unitarization of the Born term. At large $t$ our model calculations are extended up to $-t=15 \mathrm{GeV}^{2}$. We added a small contribution of the energy independent part of the spin flip amplitude in the form similar to the proposed in [78] and analyzed in [14].

$$
F_{s f}(s, t)=h_{s f} q^{3} F_{1}^{2}(t) e^{-B_{s f} q^{2}} .
$$

The energy dependent part of the spin-flip amplitude is related to the main amplitude but with an additional kinematic factor and the main slope taken twice more, conformity with the paper $[79,80]$. The form factors incoming in the spin-flip amplitude are determined by the GPD functions $H(s, t, x)$ and $E(s, t, x)$, which include the corresponding PDF distributions. The model is very simple from the viewpoint of the number of fitting parameters and functions. There are no any artificial functions or any cuts which bound the separate parts of the amplitude by some region of momentum transfer.

Now we shell restrict our discussion to the analysis of $A_{N}$ as there are some experimental data in the region of NICA energies. In the standard pictures the spin-flip and double spin-flip amplitudes correspond to the spin-orbit $(L S)$ and spin-spin $(S S)$ coupling terms. The contribution to $A_{N}$ from the hadron double spin-flip amplitudes already at $p_{L}=6 \mathrm{GeV} / \mathrm{c}$ is of the second order compared to the contribution from the spin-flip amplitude. So with the usual high energy approximation for the helicity amplitudes at small transfer momenta we suppose that $\Phi_{1}=\Phi_{3}$ and we can neglect the contributions of the hadron parts of $\Phi_{2}-\Phi_{4}$. Note that if $\Phi_{1}, \Phi_{3}, \Phi_{5}$ have the same phases, their interference contribution to $A_{N}$ will be zero, though the size of the hadron spin-flip amplitude can be large. Hence, if this phase has different $s$ and $t$ dependencies, the contribution 

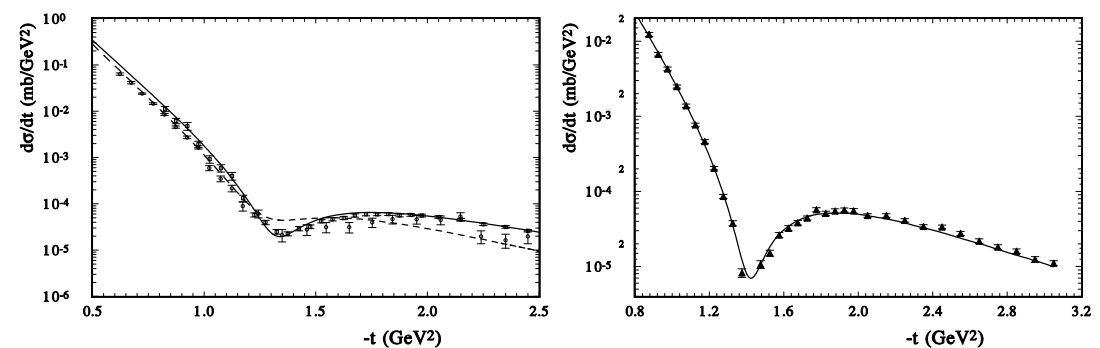

Fig. 4. The model calculation of the diffraction minimum in $d \sigma / d t$ of $p p$ scattering [left] at $\sqrt{s}=30.4 \mathrm{GeV}$; [right] for $p p$ and $p \bar{p}$ at $\sqrt{s}=52.8 \mathrm{GeV}$ [81] scattering.

from the hadron spin-flip amplitude to $A_{N}$ can be zero at $s_{i}, t_{i}$ and non-zero at other $s_{j}, t_{j}$. e experimental data $\left(\sum \chi^{2} / n_{\text {dof }}=1.24\right)$.

Now let us examine the form of the differential cross section in the region of the momentum transfer where the diffractive properties of elastic scattering appear most strongly - it is the region of the diffraction dip. The form and the energy dependence of the diffraction minimum are very sensitive to different parts of the scattering amplitude. The change of the sign of the imaginary part of the scattering amplitude determines the position of the minimum and its movement with changing energy. The contributions of the real part of the spin-non-flip scattering amplitude and the square of the spin-flip amplitude determine the size and the energy dependence of the dip. Hence, this depends heavily on the odderon contribution. The spin-flip amplitude gives the contribution to the differential cross sections additively. So the measurement of the form and energy dependence of the diffraction minimum with high precision is an important task for future experiments.

The HEGS model reproduces $d \sigma / d t$ at very small and large $t$ and provides a qualitative description of the dip region at $-t \approx 1.6 \mathrm{GeV}^{2}$, for $\sqrt{s}=10 \mathrm{GeV}$ and $-t \approx 0.45 \mathrm{GeV}^{2}$ for $\sqrt{s}=13 \mathrm{TeV}$. Note that it gives a good description for the proton-proton and protonantiproton elastic scattering or $\sqrt{s}=53 \mathrm{GeV}$ and for $\sqrt{s}=62.1 \mathrm{GeV}$ (Fig.2a).

The dependence of the position of the diffraction minimum on $t$ is determined in most part by the growth of the total cross sections and the slope of the imaginary part of the scattering amplitude. Figures $2 \mathrm{~b}$ and 3 show this a dependence obtained in the HEGS model at different energies.

In Fig.3, the description of the diffraction minimum in our model is shown for NICA energies. The HEGS model reproduces sufficiently well the energy dependence and the form of the diffraction dip. In this energy region the diffraction minimum reaches the sharpest dip at $\sqrt{s}=30 \mathrm{GeV}$ near the final NICA energy. Note that at this energy the value of $\rho(s, t=0)$ also changes its sign in the proton-proton scattering.

The calculated analyzing power at $p_{L}=6 \mathrm{GeV} / \mathrm{c}$ is shown in Fig.4a. One can see that a good description of experimental data on the analyzing power can be reached only with one hadron-spin flip amplitude.

The experimental data at $p_{L}=11.75 \mathrm{GeV} / \mathrm{c}$ seriously differ from those at $p_{L}=$ $6 \mathrm{GeV} / \mathrm{c}$ but our calculations reproduce $A_{N}$ sufficiently well (Fig.4b ). It is shown that our energy dependence of the spin-flip amplitudes was chosen correctly and we may 


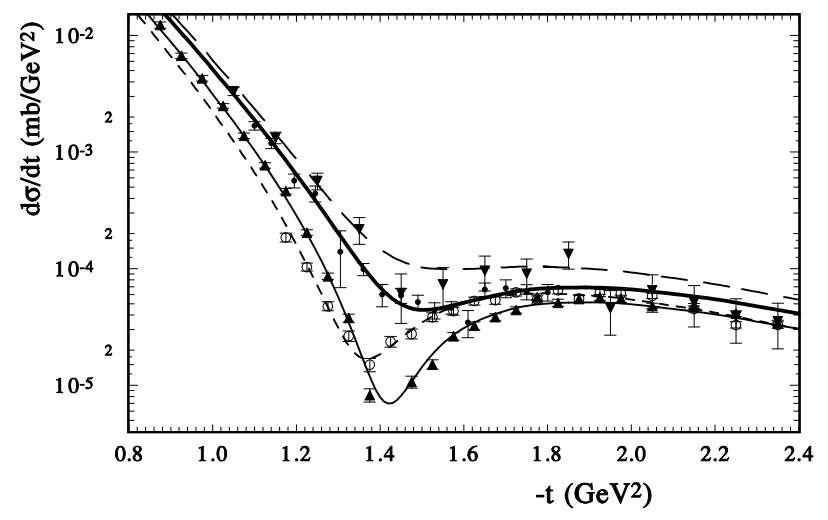

Fig. 5. The model calculation of the diffraction minimum in $d \sigma / d t$ of $p p$ at $\sqrt{s}=$ 13.4; 16.8; 30.4; 44.7; GeV; (lines, respectively, long dash; solid; thin-solid, and short - dash ); and experimental data [81], respectively, - the triangle down, the circles (solid), triangle up, and circles )
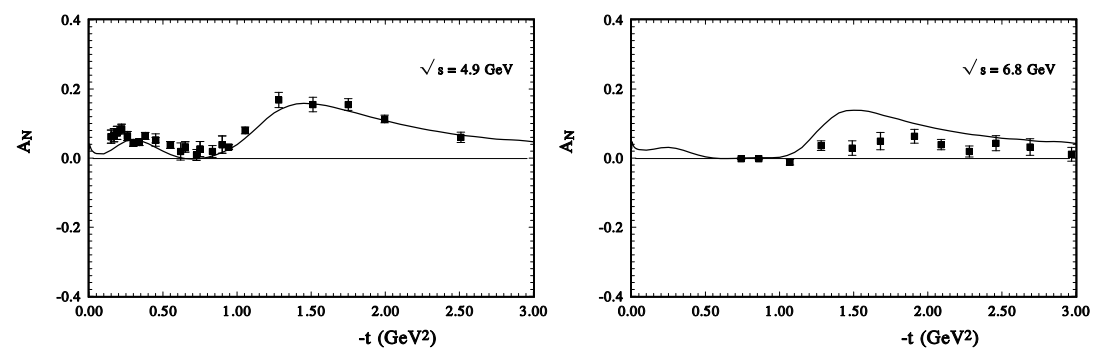

Fig. 6. The analyzing power $A_{N}$ of pp - scattering calculated: a) at $\sqrt{s}=4.9 \mathrm{GeV}$ (the experimental data [82]), and b) at $\sqrt{s}=6.8 \mathrm{GeV}$ (points - the existence experimental data [83] ). 

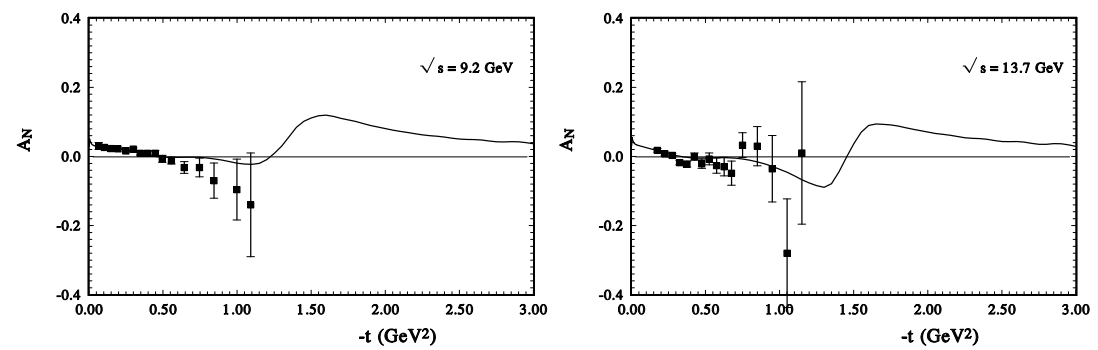

Fig. 7. The analyzing power $A_{N}$ of pp - scattering calculated: a) at $\sqrt{s}=9.2 \mathrm{GeV}$, and (the experimental data [27]), and b) at $\sqrt{s}=13.7 \mathrm{GeV}$ (points - the experimental data [84]).
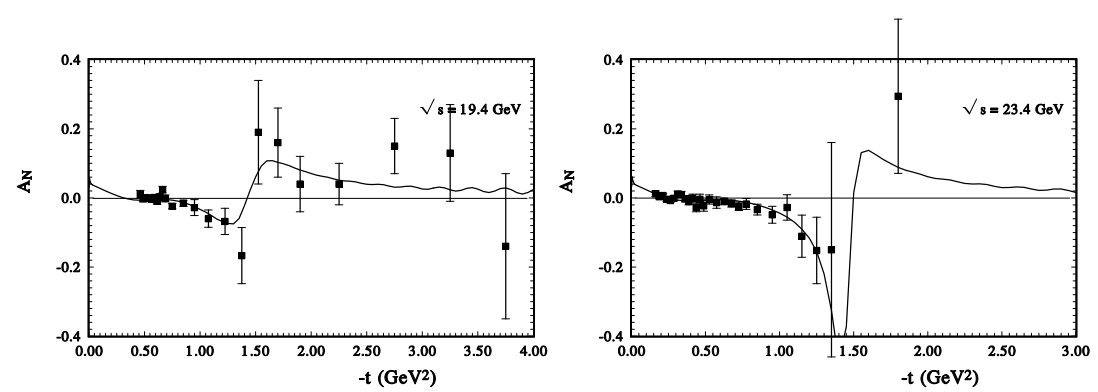

Fig. 8. The analyzing power $A_{N}$ of pp - scattering calculated: a) at $\sqrt{s}=19.4 \mathrm{GeV}$ (the experimental data [85]), and b) at $\sqrt{s}=23.4 \mathrm{GeV}$ (points - the experimental data [84])

hope that further we will obtain correct values of the analyzing power and other spin correlation parameters.

From Fig. 4 we can see that in the region $|t| \approx 0.2 \div 1 \mathrm{GeV}^{2}$ the contributions from the hadron spin-flip amplitudes are most important. At last, Fig.5a shows our calculations at $p_{L}=200 \mathrm{GeV} / \mathrm{c}$.

At this energy, the contributions of the phenomenological energy independent part of the spin-flip amplitude is compared with the energy dependent part. The spin effect is sufficiently large and has a specifical form, which is determined by the form of the differential cross section in the diffraction dip domain.

\subsection{Conclusions}

The Generelized parton distributions (GPDs) make it possible to better understand the thin hadron structure and to obtain the hadron structure in the space frame (impact parameter representations). It is tightly connected with the hadron elastic hadron form factors. The research into the form and energy dependence of the diffraction minimum of the differential cross sections of elastic hadron-hadron scattering at different energies will give valuable information about the structure of the hadron scattering amplitude and hence the hadron structure and the dynamics of strong interactions. The diffraction 
minimum corresponds to the change of the sign of the imaginary part of the spin-non-flip hadronic scattering amplitude and is created under a strong impact of the unitarization procedure. Its dip depends on the contributions of the real part of the spin-non-flip amplitude and the whole contribution of the spin-flip scattering amplitude. In the framework of HEGS model, we show a deep connection between elastic and inealastic cross sections, which are tightly connected with the hadron structure at small and large distances.

The HEGS model reproduces well the form and the energy dependence of the diffraction dip of the proton-proton and proton antiproton elastic scattering [86]. The predictions of the model in most part reproduce the form of the differential cross section at $\sqrt{s}=13 \mathrm{TeV}$. It means that the energy dependence of the scattering amplitude determined in the HEGS model and unitarization procedure in the form of the standard eikonal representation satisfies the experimental data in the huge energy region (from $\sqrt{s}=9 \mathrm{GeV}$ up to $\sqrt{s}=13 \mathrm{TeV}$. It should be noted that the real part of the scattering amplitude, on which the form and energy dependence of the diffraction dip heavily depend, is determined in the framework of the HEGS model only by the complex $\bar{s}$, and hence it is tightly connected with the imaginary part of the scattering amplitude and satisfies the analyticity and the dispersion relations. Quantitatively, for different thin structures of the scattering amplitude, a wider analysis is needed. This concerns the fixed intercept taken from the deep inelastic processes and the fixed Regge slope $\alpha^{\prime}$, as well as the form of the spin-flip amplitude. Such an analysis requires a wider range of experimental data, including the polarization data of $A_{N}(s, t), A_{N N}(s, t), A_{L L}(s, t), A_{S L}(s, t)$. The obtained information about the sizes and energy dependence of the spin-flip and double-flip amplitudes will make it possible to better understand the results of famous experiments carried out by A. Krish at the ZGS to obtain the spin-dependent differential cross sections $[87,88]$ and the spin correlation parameter $A_{N N}$ and at the AGS [89] to obtain the spin correlation parameter $A_{N}$ showing the significant spin effects at large momentum transfer. 


\section{Single-spin physics ${ }^{1}$}

Physics of single-spin processes for the SPD NICA project is proposed. This includes transverse single-spin asymmetry $\left(A_{N}\right)$ and hyperon polarization $\left(P_{N}\right)$ measurements in various types of collisions, including $p+p, d+d, \mathrm{C}+\mathrm{C}$ and $\mathrm{Ca}+\mathrm{Ca}$. The polarized $p$ - and $d$-beams in the NICA collider can be used to study $A_{N}$ for more than several dozen reactions at different energies in the $3.4<\sqrt{s}<27 \mathrm{GeV}$ range. A number of interesting phenomena have been predicted, such as the oscillation for $A_{N}\left(x_{\mathrm{F}}\right)$ and $P_{N}\left(x_{\mathrm{F}}\right)$, the resonance dependence on the energy $\sqrt{s}$ for $A_{N}$ and $P_{N}$, and the threshold dependence of $A_{N}$ on the c.m. production angle for some reactions. The role of quark composition of particles involved in the reaction is discussed.

PACS: 12.38.-t; 12.38.Qk; 13.60.-r; 13.75.-n; 13.85.-t; 13.85.Ni; 13.87.Fh;

\section{Introduction}

All previous experience in the development of spin physics testifies to its fundamental importance for understanding the laws of the micro-world, including for the construction of the theory of strong interactions. It should be noted that large values of transverse single-spin asymmetries $\left(A_{N}\right)$ and hyperon polarizations $\left(P_{N}\right)$ in a wide energy range have not yet received an unambiguous and convincing explanation within the framework of the theory of strong interactions - quantum chromodynamics (QCD), which is one of the components of the Standard Model. The experimental data accumulated to date point to a very interesting phenomenology in the field of transverse single-spin phenomena, including the nontrivial dependence of the spin observables $A_{N}$ and $P_{N}$ on the collision energy $(\sqrt{s})$, the Feynman variable $\left(x_{\mathrm{F}}\right)$, the transverse momentum $\left(p_{T}\right)$, the atomic weights of the colliding particles $\left(A_{1}\right.$ and $\left.A_{2}\right)$, the multiplicity of charged particles $\left(N_{c h}\right)$ in the event and the centrality of collisions. It is equally important to measure $A_{N}$ and $P_{N}$ for as many reactions as possible in order to understand how spin effects depend on the quark composition and other quantum characteristics of the particles involved in the reaction. Data on dozens of reactions have been accumulated, but the accuracy of measurements and the limited kinematic region in most experiments do not yet allow unambiguous conclusions to be drawn about the origin of polarization phenomena and even about their dependence on various variables. The purpose of this proposal is to significantly expand the amount of polarization data available for analysis and to improve their accuracy. This will help advance the creation of adequate models of polarization phenomena and their discrimination when compared with the entire dataset.

Planned measurements at the SPD facility in the energy range for a pair of colliding nucleons from 3.4 to $27 \mathrm{GeV}$ in the reaction c.m. frame are very important for the systematic and detailed study of polarization phenomena and the study of their dependence on various variables. Analysis of the available data within the framework of the chromomagnetic polarization of quarks (CPQ) model [90] shows that the unambiguous determination of the model parameters is possible only if there are measurements with several (three or more) values for each of the listed above variables. It should be noted that the maximum energy of the accelerator in Dubna is high enough to register particles

\footnotetext{
${ }^{1}$ This section is written by V. Abramov; E-mail: Victor.Abramov@ihep.ru
} 
with large transverse momenta in the range $p_{T}=1-4 \mathrm{GeV} / \mathrm{c}$, for which the polarization effects are significant and the quark degrees of freedom are already manifested. The identification of particles in this energy range is much easier than at large accelerators, and this is an important condition for the systematic study of polarization phenomena in a large number of reactions.

The conditions for making measurements at the SPD facility at the first stage of the NICA collider can be found in [91]. Maximum energy in the c.m. of two colliding nucleons will be $27 \mathrm{GeV}$ for $p+p$ collisions and $14 \mathrm{GeV}$ for $d+d, \mathrm{C}+\mathrm{C}$ and $\mathrm{Ca}+\mathrm{Ca}$ collisions. Vector polarization will be $50 \%$ for protons and $75 \%$ for deuterons.

Table 1. Inclusive reactions for which the spin-spin asymmetry $A_{N}$ was measured.

\begin{tabular}{|c|c|c|c|c|c|}
\hline № & Reaction & oo & Reaction & o & Reaction \\
\hline 1 & $p^{\uparrow} p(A) \rightarrow \pi^{+} X$ & 10 & $p^{\uparrow} p(A) \rightarrow J / \psi X$ & 19 & $\bar{p} d^{\uparrow} \rightarrow \pi^{0} X$ \\
2 & $p^{\uparrow} p(A) \rightarrow \pi^{-} X$ & 11 & $p^{\uparrow} p(A) \rightarrow \eta X$ & 20 & $\pi^{+} p^{\uparrow} \rightarrow \pi^{+} X$ \\
3 & $p^{\uparrow} p \rightarrow \pi^{0} X$ & 12 & $d^{\uparrow} p(A) \rightarrow \pi^{+} X$ & 21 & $\pi^{-} p^{\uparrow} \rightarrow \pi^{-} X$ \\
4 & $p^{\uparrow} p(A) \rightarrow K^{+} X$ & 13 & $d^{\uparrow} p(A) \rightarrow \pi^{-} X$ & 22 & $\pi^{-} p^{\uparrow} \rightarrow \pi^{0} X$ \\
5 & $p^{\uparrow} p(A) \rightarrow K^{-} X$ & 14 & $p^{\uparrow} p \rightarrow \Lambda X$ & 23 & $\pi^{-} d^{\uparrow} \rightarrow \pi^{0} X$ \\
6 & $p^{\uparrow} p \rightarrow K_{S}^{0} X$ & 15 & $\bar{p}^{\uparrow} p \rightarrow \pi^{+} X$ & 24 & $K^{-} d^{\uparrow} \rightarrow \pi^{0} X$ \\
7 & $p^{\uparrow} p(A) \rightarrow n X$ & 16 & $\bar{p}^{\uparrow} p \rightarrow \pi^{-} X$ & 25 & $K^{-} p^{\uparrow} \rightarrow \pi^{0} X$ \\
8 & $p^{\uparrow} p(A) \rightarrow p X$ & 17 & $\bar{p}^{\uparrow} p \rightarrow \pi^{0} X$ & 26 & $\pi^{-} p^{\uparrow} \rightarrow \eta X$ \\
9 & $p^{\uparrow} p(A) \rightarrow \bar{p} X$ & 18 & $\bar{p}^{\uparrow} p \rightarrow \eta X$ & 27 & $\bar{p} p^{\uparrow} \rightarrow \pi^{0} X$ \\
\hline
\end{tabular}

Table 1 presents 27 inclusive reactions for which there are already data on the singlespin asymmetry of hadrons [90,92]. The first 14 reactions from the Table 1 can potentially be studied at the NICA collider using the SPD facility. A list of other possible 28 reactions is shown in Table 2 and includes various particles and resonances. The initial state can be any with a polarized beam: $p^{\uparrow} p, p^{\uparrow} d, d^{\uparrow} p, d^{\uparrow} d$. Their detailed study will reveal the dependence of $A_{N}$ on kinematic and other variables, including the quark composition of the particles involved, their spin, isospin, and atomic weight.

Table 2. Inclusive reactions to be studied at the SPD for which $A_{N}$ has not yet been measured. The reaction is $p^{\uparrow} p \rightarrow h+X$. Final decay mode of the detected particle $h$ is indicated only.

\begin{tabular}{|c|c|c|c|c|c|}
\hline No & Decay mode & No & Decay mode & oo & Decay mode \\
\hline 1 & $K_{L}^{0} \rightarrow \pi^{+} \pi^{-} \pi^{0}$ & 10 & $\phi \rightarrow K^{+} K^{-}$ & 19 & $\Xi^{0} \rightarrow \Lambda \pi^{0}$ \\
2 & $\eta^{\prime} \rightarrow \pi+\pi-\eta$ & 11 & $\rho^{0}(770) \rightarrow \pi^{+} \pi^{-}$ & 20 & $\Sigma^{0} \rightarrow \Lambda \gamma$ \\
3 & $a_{0}(980) \rightarrow \eta \pi^{0}$ & 12 & $\rho^{+}(770) \rightarrow \pi^{+} \pi^{0}$ & 21 & $\Sigma^{0} \rightarrow \bar{\Lambda} \gamma$ \\
4 & $K^{0 *}(892) \rightarrow K^{+} \pi^{-}$ & 13 & $\rho^{-}(770) \rightarrow \pi^{-} \pi^{0}$ & 22 & $\Delta^{++} \rightarrow p \pi^{+}$ \\
5 & $K^{0 *}(892) \rightarrow K^{-} \pi^{+}$ & 14 & $\rho^{0}(770) \rightarrow \mu^{+} \mu^{-}$ & 23 & $\Delta^{+} \rightarrow p \pi^{0}$ \\
6 & $K^{+*}(892) \rightarrow K^{+} \pi^{0}$ & 15 & $\bar{\Lambda} \rightarrow \bar{p} \pi^{+}$ & 24 & $\Delta^{0} \rightarrow p \pi^{-}$ \\
7 & $K^{-*}(892) \rightarrow K^{-} \pi^{0}$ & 16 & $\Xi^{-} \rightarrow \Lambda \pi^{-}$ & 25 & $\Delta^{-} \rightarrow n \pi^{-}$ \\
8 & $\omega(782) \rightarrow \pi^{+} \pi^{-} \pi^{0}$ & 17 & $\Xi^{0} \rightarrow \Lambda \pi^{0}$ & 26 & $\bar{\Delta}^{--} \rightarrow \bar{p} \pi^{-}$ \\
9 & $\omega(782) \rightarrow \gamma \pi^{0}$ & 18 & $\bar{\Xi}^{+} \rightarrow \bar{\Lambda} \pi^{+}$ & 27 & $\bar{\Delta}^{0} \rightarrow \bar{p} \pi^{+}$ \\
\hline
\end{tabular}


Data on the transverse polarization of hyperons and antihyperons are no less interesting. The list of reactions available to date, for which their polarization $P_{N}$ was measured, is presented in Table 3 and includes 32 reactions [90,93]. The first 14 reactions can potentially be studied at the SPD setup. This list can be supplemented with reactions such as $p p \rightarrow \Sigma^{0 \uparrow}(1385) X, p p \rightarrow \bar{\Sigma}^{0 \uparrow} X, p p \rightarrow \Lambda^{\uparrow}(1405) X, p p \rightarrow \Lambda^{\uparrow}(1520) X$. The initial state can be any with a polarized or unpolarized beam: $p^{\uparrow} p, p^{\uparrow} d, d^{\uparrow} p, d^{\uparrow} d$ and $A A$.

Table 3. Inclusive reactions for which the polarization $\left(P_{N}\right)$ of hyperons was measured.

\begin{tabular}{|c|c|c|c|c|c|}
\hline № & Reaction & o & Reaction & o & Reaction \\
\hline 1 & $p p(A) \rightarrow \Lambda^{\uparrow} X$ & 12 & $A_{1} A_{2} \rightarrow \Lambda^{\uparrow} X$ & 23 & $\pi^{-} A \rightarrow \Xi^{-\uparrow} X$ \\
2 & $p p(A) \rightarrow \Xi^{-\uparrow} X$ & 13 & $A_{1} A_{2} \rightarrow \Lambda^{\uparrow(G)} X$ & 24 & $\pi^{-} A \rightarrow \bar{\Xi}^{+\uparrow} X$ \\
3 & $p p(A) \rightarrow \Xi^{0 \uparrow} X$ & 14 & $A_{1} A_{2} \rightarrow \bar{\Lambda}^{\uparrow}(G) X$ & 25 & $\pi^{-} p \rightarrow \Lambda^{\uparrow} X$ \\
4 & $p p(A) \rightarrow \Sigma^{+\uparrow} X$ & 15 & $\Sigma^{-} A \rightarrow \Sigma^{+\uparrow} X$ & 26 & $\pi^{-} p \rightarrow \bar{\Lambda}^{\uparrow} X$ \\
5 & $p p(A) \rightarrow \Sigma^{0 \uparrow} X$ & 16 & $\Sigma^{-} A \rightarrow \Xi^{-\uparrow} X$ & 27 & $\pi^{+} p \rightarrow \Lambda^{\uparrow} X$ \\
6 & $p p(A) \rightarrow \Sigma^{-\uparrow} X$ & 17 & $\Sigma^{-} A \rightarrow \Lambda^{\uparrow} X$ & 28 & $K^{-} A \rightarrow \Xi^{-\uparrow} X$ \\
7 & $p p(A) \rightarrow \Omega^{-\uparrow} X$ & 18 & $\Sigma^{-} A \rightarrow \bar{\Lambda}^{\uparrow} X$ & 29 & $\bar{p} A \rightarrow \bar{\Lambda}^{\uparrow} X$ \\
8 & $p p(A) \rightarrow \bar{\Lambda}^{\uparrow} X$ & 19 & $K^{-} p \rightarrow \Lambda^{\uparrow} X$ & 30 & $e^{+} e^{-} \rightarrow \Lambda^{\uparrow} X$ \\
9 & $p p(A) \rightarrow \bar{\Xi}^{+\uparrow} X$ & 20 & $K^{-} p \rightarrow \bar{\Lambda}^{\uparrow} X$ & 31 & $\nu_{\mu} A \rightarrow \Lambda^{\uparrow} X$ \\
10 & $p p(A) \rightarrow \bar{\Xi}^{0 \uparrow} X$ & 21 & $K^{+} p \rightarrow \Lambda^{\uparrow} X$ & 32 & $e^{+} A \rightarrow \bar{\Lambda}^{\uparrow} X$ \\
11 & $p p(A) \rightarrow \bar{\Sigma}^{-\uparrow} X$ & 22 & $K^{+} p \rightarrow \bar{\Lambda}^{\uparrow} X$ & 33 & - \\
\hline
\end{tabular}

It is important to note that for hyperons it is possible to simultaneously measure both the transverse polarization $P_{N}$ and the single-spin asymmetry $A_{N}$. Comparing $A_{N}$ and $P_{N}$ for a specific reaction with the predictions of various models will bring us closer to revealing the mechanism of the origin of polarization phenomena at high energies and will shed light on the physics of strong interactions in the confinement region.

A systematic study of polarization data assumes the presence of a model that describes, within a single mechanism, a large number of reactions depending on the variables listed above. An example of such a model is the model of chromomagnetic polarization of quarks (CPQ) [90].

References to most of publications devoted to polarization experiment data can be found in $[90,92,93]$ and other in papers listed in the cited literature. The following sections describe in more detail the model of chromomagnetic polarization of quarks and consider examples of existing data and calculations of $A_{N}$ and $P_{N}$ for various reactions that can potentially be studied using the SPD setup at the NICA collider in Dubna.

\subsection{Model of chromomagnetic polarization of quarks}

The phenomenological model of chromomagnetic polarization of quark is based on the following basic assumptions [90]:

1) As a result of collisions of hadrons, a pair of quarks with a large transferred transverse momentum $p_{T}$ is scattered. Further, the scattered (test) quark with large $p_{T}$ moves in the effective chromomagnetic field $\mathbf{B}^{a}$ and experiences the action of the 
Stern-Gerlach force proportional to the product of the field gradient components and the corresponding components of the quark chromomagnetic moment. The direction of the Stern-Gerlach force and the additional transverse momentum received by the test quark in the effective chromomagnetic field depend on the projections of the quark spin onto the quantization axis. Subsequently, the polarized quark from the incident polarized proton recombines with other quarks to form the observed hadron. The angular distribution of such hadrons has an azimuthal dependence, i.e., a single-spin asymmetry arises. If unpolarized hadrons collide, then the action of the Stern-Gerlach force imparts an additional transverse momentum directed to the left or to the right, depending on the direction of the projection of the quark spin up or down, when the quark moves, for example, to the left. Thus, when scattering to the left, a quark has predominantly one polarization sign, and when scattering to the right, the opposite. The hyperons formed from these quarks acquire transverse polarization relative to the scattering plane.

2) The effective chromomagnetic field $\mathbf{B}^{a}$ is created by spectator quarks, that is, all quarks that will not be included in the recorded hadron. Spectator quarks are moving in the c.m. in the direction of the colliding hadrons and create for a short time a circular transverse chromomagnetic field. The sign of the circular chromomagnetic field to the left and to the right of the collision axis is opposite, but the field gradient does not change its direction, which ensures a nonzero polarization effect due to the action of the SternGerlach force. The predominant direction of polarization of quarks in a chromomagnetic field arises, hence the name of the model.

3) When taking into account the interaction of a test quark with the field created by a moving spectator quark, it is necessary to take into account the color factor for the corresponding pair of quarks (spectator and test quarks). An analysis of the data showed that the quark-antiquark pair interacts predominantly in the color-singlet state with the color factor $C_{F}=4 / 3$, and the quark-quark or antiquark-antiquark pair interacts in the color-triplet state with $C_{F}=2 / 3$. For a hydrogen-like potential, the wave function of two quarks or a quark and an antiquark at zero coordinate is proportional to $|\psi(0)| \propto$ $\left(C_{F} \alpha_{S}\right)^{3 / 2}$ [94], which leads to the ratio of contributions from $q q$ and $q \bar{q}$ interactions to an effective field of the order

$$
\lambda \approx-\left|\psi_{q q}(0)\right|^{2} /\left|\psi_{q \bar{q}}(0)\right|^{2}=-1 / 8=-0.125 .
$$

The minus sign in (14) takes into account the opposite sign of the field created by a moving spectator quark and a moving spectator antiquark. Experimentally, the value of the global parameter, obtained as a result of the global fit of the polarization data, turned out to be $\lambda=-0.1363 \pm 0.0003$.

If the spectator quark is a product of target fragmentation and moves in the c.m. in the opposite direction, then its contribution to the effective field will be additionally suppressed by the factor $-\tau$, where $\tau=0.0267 \pm 0.0012$ is another important global parameter of the CPQ model. This suppression of the contribution of quarks from the target is due to the fact that the chromomagnetic field they create is in a different region of space-time and, therefore, has almost no effect on the test quarks moving forward.

4) The presence of an effective chromomagnetic field should lead to the precession of the test quark spin when it moves in the field. Analysis of the data showed that the effective field length and the corresponding precession angle are proportional to $x_{A}=$ 
$\left(x_{R}+x_{\mathrm{F}}\right) / 2$ and $x_{B}=\left(x_{R}-x_{\mathrm{F}}\right) / 2$ in the fragmentation region of the incident particle $\mathrm{A}$ and target $\mathrm{B}$, respectively. As a result, this leads to oscillations of the dependence of $A_{N}$ and $P_{N}$ on the kinematic variables $x_{A}$ and $x_{B}$, and hence on $x_{\mathrm{F}}$ and $p_{T}$. These oscillations are the main feature of the CPQ model and should manifest themselves in the case of strong fields, when the precession angles reach values of the order of $\pi$ or more.

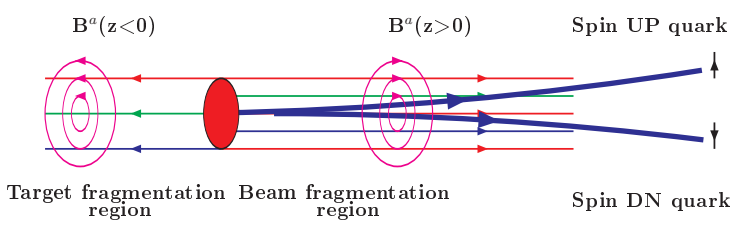

Fig. 9. The mechanism of origin of single-spin polarization phenomena.

The mechanism of origin of single-spin polarization phenomena is shown schematically in fig. 9. The interaction of colliding particles $A$ and $B$ is considered in the c.m. of pair of colliding nucleons.

Observables $A_{N}$ and $P_{N}$ are described by equations (15) and (16):

$$
\begin{gathered}
P_{N}=C(\sqrt{s}) F\left(p_{T}, A\right)\left[G\left(\phi_{A}\right)-\sigma G\left(\phi_{B}\right)\right], \\
G(\phi)=(1-\cos \phi) / \phi+\epsilon \cdot \phi,
\end{gathered}
$$

where function (16) takes into account the action of the Stern-Gerlach forces and the precession of the quark spin, and where $\epsilon=-0.00497 \pm 0.00009$ is the global parameter of the CPQ model, $\sigma$ is the local parameter. The integral angles of precession of the quark spin are

$$
\phi_{A}=\omega_{A}^{0} y_{A}, \quad \phi_{B}=\omega_{B}^{0} y_{B},
$$

in the fragmentation region of colliding particles $A$ and $B$, respectively. The oscillation frequency $\omega_{A(B)}^{0}$ is described by the equation

$$
\omega_{A(B)}^{0}=g_{s} \alpha_{s} \nu_{A(B)} m_{r}\left(g_{Q}^{a}-2\right) / M_{Q},
$$

where $\alpha_{s}=g_{s}^{2} /(4 \pi)$ is the current strong interaction constant, $g_{s}$ is the color charge, $M_{Q}$ is the mass of the constituent quark $Q, g_{Q}^{a}$ is the the Lande gyromagnetic color factor of a quark, $m_{r}=0.2942 \pm 0.0072 \mathrm{GeV}$ is the global parameter that can be considered as the ratio of the maximum longitudinal extension of the chromomagnetic field to the square of its radius.

The total contribution of spectator quarks (with weights $\lambda$ and $-\tau$ ) to $\nu_{A(B)}$ in the fragmentation region of colliding particles $A$ and $B$, respectively, is calculated using quark diagrams and the quark counting rules [90].

Quark diagrams for the reactions $p^{\uparrow}+p \rightarrow \pi^{+}+X$ and $p^{\uparrow}+p(A) \rightarrow p+X$ are shown in fig. 10a and 10b, respectively. When nucleus is the target, as in the case of fig. 10b, the number of target spectator quarks is equal to $3 A_{\text {eff }} \propto A^{1 / 3}$, where $A$ is an atomic weight, since all target quarks hit by the incident proton contribute to the spectator quarks [90]. Below we assume $A_{\text {eff }}=A=1$. 
In the approximation of moderate energies $(\sqrt{s}<70 \mathrm{GeV})$, we obtain $\nu_{A}$ for the reaction $p^{\uparrow}+p \rightarrow \pi^{+}+X$ :

$$
\nu_{A}=\nu_{B}=3 \lambda-3 \tau \lambda A_{\mathrm{eff}}=-0.398
$$

and for the reaction $p^{\uparrow}+p(A) \rightarrow p+X$ :

$$
\nu_{A}=\nu_{B}=2+2 \lambda-3 \tau \lambda A_{\mathrm{eff}}=1.738 \text {. }
$$

To calculate $\nu_{A}$ we have to add up all the contributions $(\nu)$ of the spectator quarks shown to the right of the quark diagram. The $\nu_{A}$ value for the reaction $p^{\uparrow}+p \rightarrow \pi^{+}+X$ is much less than 1 in absolute value. Consequently, the oscillation frequency $\omega_{A(B)}^{0}$ is also low, and the $A_{N}\left(x_{\mathrm{F}}\right)$ dependence is close to linear. For the reaction $p^{\uparrow}+p(A) \rightarrow p+X$, the value of $\nu_{A}$ is significantly greater than unity in absolute value, and for it, as we will see below, a nonmonotonic oscillating dependence $A_{N}\left(x_{\mathrm{F}}\right)$ is indeed observed.

Kinematic variables

$$
\begin{aligned}
& y_{A}=x_{A}-\left(E_{0} / \sqrt{s}+f_{0}\right)\left[1+\cos \theta_{c m}\right]+a_{0}\left[1-\cos \theta_{c m}\right], \\
& y_{B}=x_{B}-\left(E_{0} / \sqrt{s}+f_{0}\right)\left[1-\cos \theta_{c m}\right]+a_{0}\left[1+\cos \theta_{c m}\right],
\end{aligned}
$$

are expressed in terms of the scaling variables $x_{A}$ and $x_{B}$, the reaction energy $\sqrt{s}$, the emission angle $\theta_{c m}$ in c.m. and three local parameters $E_{0}, a_{0}$ and $f_{0}$. Function

$$
C(\sqrt{s})=v_{0} /\left[\left(1-E_{R} / \sqrt{s}\right)^{2}+\delta_{R}^{2}\right]^{1 / 2}
$$

takes into account the dependence of the rate of precession of the spin of a quark on its energy $E_{Q}$ in c.m. and the effect of attraction $\left(E_{R}>0\right)$ or repulsion $\left(E_{R}<0\right)$ between the test quark and spectator quarks. The $E_{R}$ sign is determined by the factor $-g_{S} \nu_{A}$, where $g_{S}$ is the color charge of the test quark (positive for a quark and negative for an antiquark). An example of a reaction with $E_{R}>0$ is $p+p \rightarrow \bar{\Lambda}+X$, and reaction with $E_{R}<0$ is $p+p \rightarrow \Lambda+X$. The global data fit confirms the $E_{R}$ sign rule for most of 85 investigated reactions $(96,5 \%)$.

The coefficient $v_{0}$ determines the value of $A_{N}$ and $P_{N}$ and is calculated as follows:

$$
v_{0}=-D_{r} g_{Q}^{a} P_{Q} / 2\left(g_{Q}^{a}-2\right),
$$

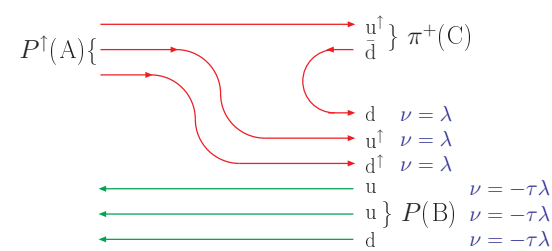

(a)

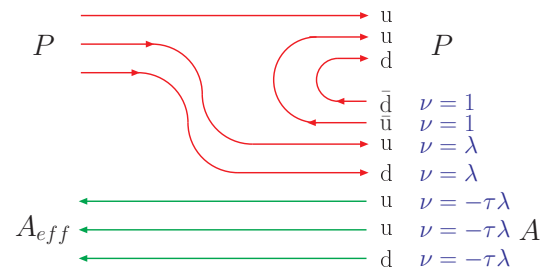

(b)

Fig. 10. Quark flux diagrams for the reaction $p^{\uparrow}+p \rightarrow \pi^{+}+X$ (a) and $p^{\uparrow}+p(A) \rightarrow p+X$ (b). 
where $D_{r}$ is a local dimensionless parameter of order 0.8 , which is the ratio of the spectrum slope in $p_{T}$ to the transverse radius of the effective field, $P_{Q}$ is the polarization of the $Q$ quark in a polarized proton ( +1 for $u$-quark and -1 for $d$-quark), $g_{Q}^{a}$ is the Lande gyromagnetic factor for the $Q$-type quark, which is a global parameter. The $A_{N}$ or $P_{N}$ sign for most reactions at small $\phi_{A}$ is the product of three factors: $-g_{S} \nu_{A} P_{Q}$. When calculating the polarization of hyperons, we set $P_{Q}=1$.

The color form factor $F\left(p_{T}, A\right)$ suppresses $A_{N}$ and $P_{N}$ at low $p_{T}$, when the colored quarks inside the hadron are not visible due to the uncertainty relation:

$$
F\left(p_{T}, A\right)=1-\exp \left[-\left(p_{T} / p_{T}^{0}\right)^{2.5}\right]\left(1-\alpha_{A} \ln A\right),
$$

where $p_{T}^{0}$ is a local parameter, and the other parameter $\alpha_{A}$ is zero for most of reactions.

The dependence of a number of parameters on the atomic masses $A_{1}$ and $A_{2}$ turned out to be universal for most of the reactions in Tables 1 and 3 [90,95]. Further development of the CPQ model is reflected in papers [95-104].

\subsection{Single-spin hadron asymmetry}

The most numerous experiments to measure the single-spin asymmetry were carried out for the reactions of the production of charged and neutral $\pi$-mesons in $p^{\uparrow} p$ and $p^{\uparrow} A$ collisions. These data are included in the general database of polarization phenomena, which contains 3608 experimental points for 85 different inclusive reactions, in which the polarization of one of the particles is known or measured in the initial or final state $[90,102]$. A global fit was performed for the entire dataset using the CPQ model.

Data on $A_{N}$ for the reaction $p^{\uparrow}+p(A) \rightarrow \pi^{+}+X$ at different energies are shown in fig. 11a from [102], where they are compared with the results of calculations using the CPQ model. As seen from fig. 11a, $A_{N}\left(x_{\mathrm{F}}\right)$ dependence for the reactions $p^{\uparrow}+p(A) \rightarrow$ $\pi^{+}+X$ at moderately high energies $\sqrt{s}<70 \mathrm{GeV}$, is almost linear, which agrees with the predictions of the CPQ model. This is due to the insignificant value of the parameter $\nu_{A}=\nu_{B}=3 \lambda-3 \tau \lambda=-0.398$, which follows from the quark diagram shown in fig. 10a.

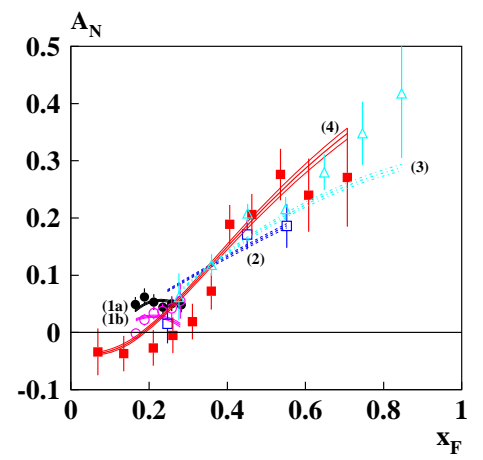

(a)

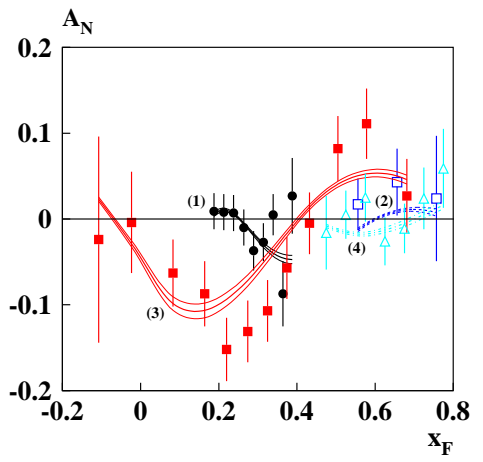

(b)

Fig. 11. $A_{N}\left(x_{\mathrm{F}}\right)$ for the reaction $p^{\uparrow}+p(A) \rightarrow \pi^{+}+X$ (a) and $p^{\uparrow}+p(A) \rightarrow p+X$ [102] (b) [102]. 
The positive sign of $A_{N}\left(x_{\mathrm{F}}\right)$ for the reaction $p^{\uparrow}+p(A) \rightarrow \pi^{+}+X$ is explained by the dominant contribution of the positively polarized test $u$-quark from a polarized proton.

A very unexpected and interesting feature of the reaction $p^{\uparrow}+p(A) \rightarrow \pi^{-}+X$ turned out to be the threshold dependence of $A_{N}\left(y_{A}\right)$ on the angle of production $\theta_{c m}$ in c.m. In fig. 12a from [96] is shown the dependence of the quantity $\left(1-E_{R} / \sqrt{s}\right) A_{N}$ on $y_{A}$, where $E_{R}=4.98 \pm 0.29 \mathrm{GeV}$. It turned out that this quantity is described by the universal function of $y_{A}$ if $\theta_{c m}<74^{\circ}$, and is equal to zero if $\theta_{c m}>74^{\circ}$. In fig. 12a, two clearly distinct branches are visible, into which the experimental points are grouped.

Within the framework of the CPQ model, the threshold effect for $A_{N}\left(y_{A}\right)$ can be qualitatively explained by the greater mass of the constituent $d$-quark as compared to the mass of the $u$-quark.

In fig. $12 \mathrm{~b}$ from [96] is shown the dependence of the quantity $\left(1-E_{R} / \sqrt{s}\right) A_{N}$ on $y_{A}$ for the reaction $p^{\uparrow}+p(A) \rightarrow \pi^{+}+X$, where $E_{R}=1.92 \pm 0.30 \mathrm{GeV}$. Most of the light test $u$-quarks flying into the front hemisphere will be from a polarized proton, which means that the asymmetry $A_{N}>0$ for $\pi^{+}$mesons [96]. All data in fig. 12b are located on the same branch, for a wide range of energies $\sqrt{s}$ and production angles in c.m.

The positive value $E_{R}=4.98 \pm 0.29 \mathrm{GeV}$ for the reaction $p^{\uparrow}+p(A) \rightarrow \pi^{-}+X$, found in the framework of the CPQ model, is a manifestation of the effect of "attraction" of test quarks and spectator quarks. According to formula (23), $A_{N}$ reaches its maximum value at energy $\sqrt{s} \approx E_{R}[90,96]$. Investigation of the effect of "attraction" of test quarks for various reactions is one of the objectives of this proposal and involves scanning in energy $\sqrt{s}$ near $E_{R}$. This phenomenon is observed not only for single-spin asymmetry, but also for the polarization of hyperons, in those reactions for which $E_{R}$ is positive and amounts to several $\mathrm{GeV}[90]$.

Finding of scaling (independence of $\left(1-E_{R} / \sqrt{s}\right) A_{N}$ from energy $\sqrt{s}$ ) in the variable

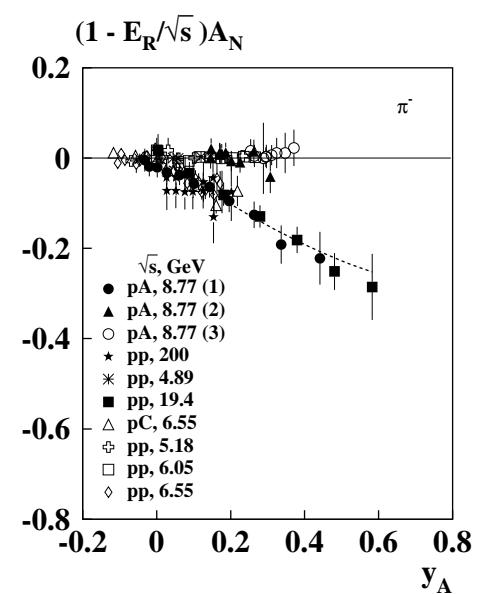

(a)

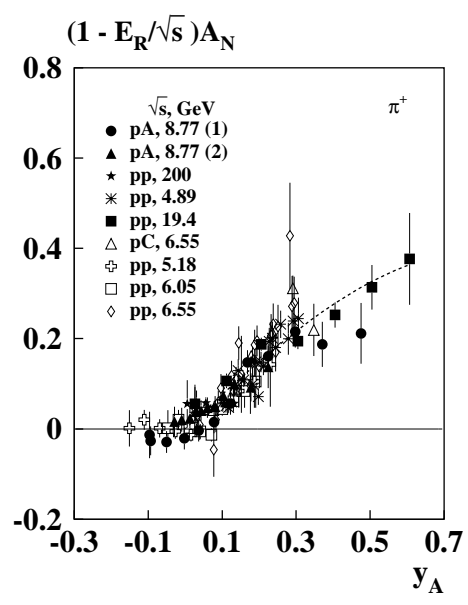

(b)

Fig. 12. Dependence of the value $\left(1-E_{R} / \sqrt{s}\right) A_{N}$ on $y_{A}$, where $E_{R}=4.98 \pm 0.29 \mathrm{GeV}$ for the reaction $p^{\uparrow} p(A) \rightarrow \pi^{-} X$ (a) and $E_{R}=1.92 \pm 0.30 \mathrm{GeV}$, for the reaction $p^{\uparrow} p(A) \rightarrow \pi^{+} X$ (b) [96]. 
$y_{A}$ was one of the stages in the process of creating the CPQ model $[90,92,96]$. Investigation of the scaling for polarization observables $A_{N}$ and $P_{N}$ is of independent interest and can be one of the tasks for the SPD setup. In the framework of the CPQ model, scaling in polarization phenomena is due to the occurrence of processes at the quark level, in the limit of high energies and large transverse momentum [90,92,93,96].

Data and calculations of $A_{N}\left(x_{\mathrm{F}}\right)$ for the reaction $p^{\uparrow}+p(A) \rightarrow p+X$, taken from [102], are shown in fig. 11b. The data of the FODS-2 experiment [105], measured in a wide range in the variable $x_{\mathrm{F}}$, at an energy of $\sqrt{s}=8.77 \mathrm{GeV}$ (solid squares in fig. 11b and curve (3)), demonstrate a nonmonotonic oscillatory dependence of $A_{N}\left(x_{\mathrm{F}}\right)$. This is a consequence of the large value of the parameter $\nu_{A}$ and the significant precession angle of the quark spin in the chromomagnetic field. The quantity $\nu_{A}=\nu_{B}=[2+2 \lambda-3 \tau \lambda]=1.738$ is large enough, which follows from the quark diagram shown in fig. 10b. In the energy region of the NICA collider, a negative asymmetry $A_{N}\left(x_{\mathrm{F}}\right)$ of about $10 \%$ is expected near $x_{\mathrm{F}}=0.2$ (fig. $11 \mathrm{~b}$, curves $(3)$ for $\sqrt{s}=8.77 \mathrm{GeV}$ ).

Another new and interesting direction in the study of polarization phenomena is associated with the dependence of $A_{N}$ and $P_{N}$ on the multiplicity of charged particles $\left(N_{c h}\right)$ in an event. The first results in this region were obtained for reactions $p^{\uparrow}+p \rightarrow$ $\pi^{ \pm}+X$ in the BRAHMS experiment at an energy $\sqrt{s}=200 \mathrm{GeV}$ [106]. The single-spin asymmetry $A_{N}$ increases in absolute value, if we select events with $N_{c h}$ above the average, and decreases, if we select events with $N_{c h}$ below the average. These data, together with the calculations, are disscussed in [100]. In the CPQ model, events with a multiplicity above the mean correspond to quark diagrams with additional quark-antiquark pairs compared to the minimum required number. This effect, which can manifest itself for both $A_{N}$ and $P_{N}$, can be studied at the SPD facility.

\subsection{Transverse polarization of hyperons}

Hyperons have the remarkable property that their decay in the weak interaction makes it possible to determine the transverse polarization to the scattering plane $\left(P_{N}\right)$ - the only one possible in strong interactions, due to the conservation of parity in them. Therefore, the polarization of hyperons can be studied in collisions of practically any particles. In the case of the first phase of the SPD NICA project, we are interested in collisions $p p$, $p d, d d, \mathrm{C}+\mathrm{C}$ and $\mathrm{Ca}+\mathrm{Ca}$. The available data are discussed in detail in [93].

Quark diagrams for the production of $\Xi^{-}$hyperons in pp-collisions can be found in [104]. The effective number of spectator quarks for the reaction $p+p \rightarrow \Xi^{-\uparrow}+X$ is $\nu_{A}=\nu_{B}=2+2 \lambda-3 \tau \lambda \approx 1.7383$. Similar calculations for the reaction $p+p \rightarrow \Lambda^{\uparrow}+X$ give $\nu_{A}=\nu_{B}=1+\lambda-3 \tau \lambda \approx 0.8746$. Therefore, a nonmonotonic dependence $P_{N}\left(x_{\mathrm{F}}\right)$ can be expected in the case of the reaction $p+p \rightarrow \Xi^{-\uparrow}+X$.

The $P_{N}\left(x_{\mathrm{F}}\right)$ data for the reaction $p+p(A) \rightarrow \Lambda^{\uparrow}+X$ are shown in fig. 13a, and the data for the reaction $p+p(A) \rightarrow \Xi^{-\uparrow}+X$ are shown in fig. 13b, together with the CPQ model predictions [104].

As seen from fig. 13b, the $P_{N}\left(x_{\mathrm{F}}\right)$ dependence for cascade hyperons is nonlinear function and $P_{N}\left(x_{\mathrm{F}}\right)$ reaches its maximum absolute value at $x_{\mathrm{F}}$ in the range $0.5-0.6$, in agreement with the calculations by the CPQ model. For the reaction $p+p(A) \rightarrow \Lambda^{\uparrow}+X$, a close to linear dependence is observed, since the parameter $\nu_{A}=\nu_{B} \approx 0.8746$ in this case 
is approximately two times smaller. The maximum of the absolute value of polarization for the reaction $p+p(A) \rightarrow \Lambda^{\uparrow}+X$ is approximately twice that for $p+p(A) \rightarrow \Xi^{-\uparrow}+X$, and continues to increase with increasing $x_{\mathrm{F}}$ up to 0.75 , in agreement with the calculations in the framework of the $\mathrm{CPQ}$ model.

Detailed calculations of $P_{N}\left(x_{\mathrm{F}}\right)$ for reactions $p+A \rightarrow \Xi^{-}+X$ and $p+A \rightarrow \Xi^{0}+X$ can be found in [104], which also covers the energy range, available at the NICA collider.

The highest oscillation frequency $P_{N}\left(x_{\mathrm{F}}\right)$ is expected, according to calculations by the $\mathrm{CPQ}$ model, in the reactions of antibaryon production in baryon collisions. This is due to the large number of spectator quarks from projectile (there are 6 of them, see fig. 14a) accompanying the production of three antiquarks, which make up an antibaryon. There is a very limited set of data on the polarization $P_{N}\left(x_{\mathrm{F}}\right)$ of antihyperons produced in nucleonnucleon collisions. In fig. 14a is shown quark diagram for the reaction $p+A \rightarrow \bar{\Xi}^{+}+X$. The weighted number of spectator quarks for both reactions is $\nu_{A}=\nu_{B}=6-3 \tau A_{\text {eff }} \approx$ 5.92. This leads to a high oscillation frequency $P_{N}\left(x_{\mathrm{F}}\right)$ according to (18), so that in the range $0<x_{\mathrm{F}}<1$, several complete cycles can be observed.

In fig. $14 \mathrm{~b}$ are shown the data for the reaction $p+A \rightarrow \bar{\Xi}^{+}+X$ [107]. There are also shown there the calculations of $P_{N}\left(x_{\mathrm{F}}\right)$ according to the CPQ model [104]. Although the available data agree with the calculations of $P_{N}\left(x_{\mathrm{F}}\right)$ using the CPQ model, the number of experimental points are clearly insufficient to prove the phenomenon of $P_{N}\left(x_{\mathrm{F}}\right)$ oscillations. New additional data are required in the range $0<x_{\mathrm{F}}<1$ to observe several cycles of $P_{N}\left(x_{\mathrm{F}}\right)$ oscillations. Examples of $P_{N}\left(x_{\mathrm{F}}\right)$ calculations for the reactions $p+A \rightarrow \bar{\Xi}^{+}+X$ and $p+A \rightarrow \bar{\Xi}^{0}+X$ can be found in [104].

The effect of "attraction" in the polarization of antihyperons should manifest itself most clearly in the reaction $p+A \rightarrow \bar{\Lambda}+X$ [101]. The dependence of $P_{N}$ on the energy $\sqrt{s}$ of the resonance type is expected, with a maximum at $\sqrt{s}=E_{R}=6.98 \mathrm{GeV}$. This behavior $P_{N}(\sqrt{s})$ is based on a single non-zero $P_{N}$ report for reaction $p+A \rightarrow \bar{\Lambda}+X$, observed in experiment E766 at $\sqrt{s}=7.31 \mathrm{GeV}$ [108]. It is very important to repeat such measurements that are within the energy range available at the NICA collider in $p+p$, $d+d, \mathrm{C}+\mathrm{C}$ and $\mathrm{Ca}+\mathrm{Ca}$ collisions. The width of the "resonant" peak is small, since the

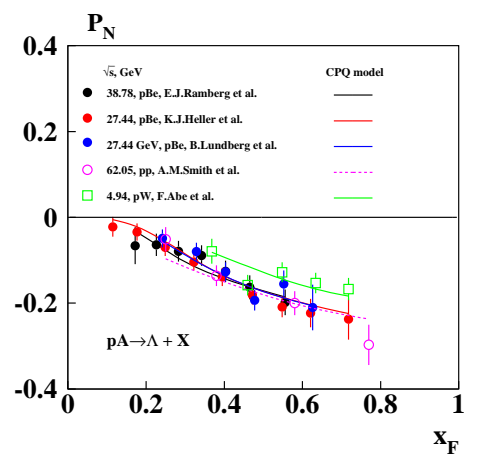

(a)

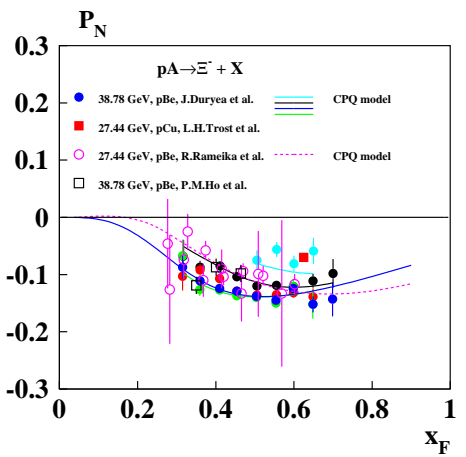

(b)

Fig. 13. $P_{N}\left(x_{\mathrm{F}}\right)$ data and the CPQ model calculations for the reaction $p+p(A) \rightarrow \Lambda^{\uparrow}+X$ (a) and $p+p(A) \rightarrow \Xi^{-\uparrow}+X(\mathbf{b})$, taken from [104]. 
precession of only one test $\bar{s}$-quark is important in this case [101]. In case of the reaction $p+A \rightarrow \bar{\Xi}^{+}+X$, there are two $\bar{s}$-quarks and one $\bar{d}$-quark with different precession frequencies, which broadens the "resonant" peak.

Investigation of the dependences $P_{N}(\sqrt{s})$ of the "resonant" type and $P_{N}\left(x_{\mathrm{F}}\right)$ of the "oscillating" type for the reaction $p+A \rightarrow \bar{\Lambda}+X$ is a very interesting problem affecting many aspects of strong interactions, such as color forces between quarks, precession of quark spin in a chromomagnetic field, quark counting rules for spectator quarks creating the field, anomalous chromomagnetic moment of quarks, the role of constituent (dressed) quarks in hadron interaction and formation and quark confinement phenomenon.

An example of possible studies of $P_{N}$ in collisions of ions can be found in [98]. It is shown that the higher is the atomic weight of the ions, the higher is the frequency of the oscillations, since the effective chromomagnetic field is increased by the quarks, coming from colliding ions.

The only available data for the $A+A \rightarrow \Lambda X$ reaction in heavy ion collisions, where $P_{N}$ was measured, were used as input to the CPQ model. The data were obtained in a fixed-target experiment, where $\Lambda$ was produced in $\mathrm{Au}+\mathrm{Au}$ collisions at c.m. energy $\sqrt{s}=4.86 \mathrm{GeV}[109]$.

Already at the first stage of the SPD NICA project, it is possible to start studing the transverse polarization of hyperons and antihyperons in ion collisions. We also note the possibility of simultaneous measurement of the so-called global polarization with respect to the reaction plane. In this case the rotation of hadron or quark matter after collision of two nuclei leads to the hyperon polarization with respect to the reaction plane, determined by an impact parameter.

To conclusion, the study of single-spin polarization phenomena in the SPD NICA project makes it possible to reveal the regularities in the behavior of the single-spin asymmetry of hadrons and the transverse polarization of hyperons and antihyperons. Such studies are possible due to the $4 \pi$ geometry of the SPD facility, a developed identification system, a fairly wide range of available energies, the presence of beams of polarized protons and deuterons, as well as ion beams. Among the most interesting tasks on this

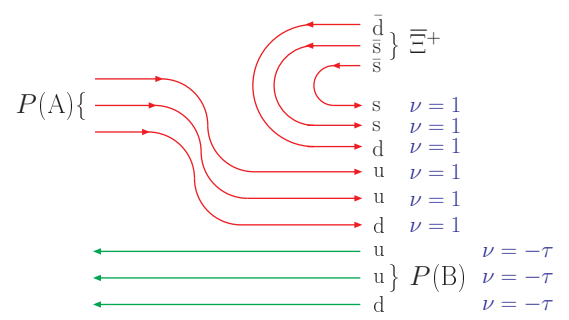

(a)

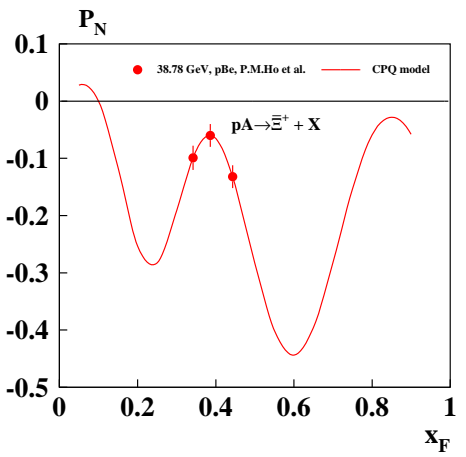

(b)

Fig. 14. Quark flow diagram (a) and $P_{N}\left(x_{\mathrm{F}}\right)$ data [107] (b) for the reaction $p+\mathrm{Be} \rightarrow \bar{\Xi}^{+}+\mathrm{X}$, taken from [104]. 
topic are the following:

1) Measurement of $A_{N}$ and $P_{N}$ at several energies $\sqrt{s}$ in a wide range for $x_{F}$ and $p_{T}$, in order to separate the dependences on these three kinematic variables. The form of these dependences reflects the mechanism of the origin of polarization phenomena. These measurements should be carried out for as many reactions as possible, which is important for studying the dependence of $A_{N}$ and $P_{N}$ on the type of particles participating in the reaction. In general, this study will significantly expand the database available for theoretical analysis and discrimination of theoretical models.

2) Investigation of the scaling phenomenon for $A_{N}$ and $P_{N}$ and corrections to it, reflecting the peculiarities of the mechanism of the origin of polarization phenomena.

3)Investigation of the threshold phenomena for $A_{N}$, including the measurement of the threshold angle of hadron production in the c.m. on which $A_{N}$ becomes null.

4) Investigation of the phenomenon of $A_{N}$ and $P_{N}$ oscillations and the relationship between the oscillation frequency and the number of spectator quarks and the type of hadrons participating in the reaction. Particularly interesting in this respect are antihyperons and cascade hyperons, as well as secondary protons and neutrons, for which the oscillation frequency reaches a significant value, which facilitates its measurement. High oscillation frequency is expected also in heavy ion collisions.

5) Investigation of the phenomenon of "resonance" dependence of $A_{N}$ and $P_{N}$ on energy $\sqrt{s}$. Disclosure of the mechanism of this phenomenon.

6) Study of the dependence of $A_{N}$ and $P_{N}$ on the atomic weights of the particles involved in collisions. This will allow not only to link the data obtained with different nuclei, but also to use the nuclei as tools for investigating the mechanism underlying the polarization phenomena. Research using ion collisions will provide a new insight into the phenomena previously studied in hadron-hadron collisions. Until now there is only one experiment in which the transverse polarization of a hyperon was measured in heavy ion collisions. Global polarization with respect to the reaction plane can be measured in addition to the $P_{N}$, which is measured with respect to the production plane.

7) Additional possibilities for studying the mechanism of polarization phenomena are provided by the use of such variables as the multiplicity of charged particles in an event, as well as the centrality of collisions and the impact parameter in the case of collisions of nuclei.

The data obtained in the proposed studies will significantly expand the general world database on polarization measurements and become the basis for their systematic theoretical analysis, within the framework of a unified approach. One of the models that makes it possible to carry out a systematic global analysis of polarization data is the model of chromomagnetic polarization of quarks, which makes it possible to analyze various reactions in a wide range of kinematic and other variables that determine the experimental conditions. Global analysis of the entire dataset is suggested. 


\section{Vector light and charmed meson production ${ }^{1}$}

In the context of NICA-SPD project, the motivation of the study of vector meson, charm production (hidden) $p+p \rightarrow p+p+V, V=\rho, \phi, J / \Psi$ and open $N+N \rightarrow \Lambda_{C}\left(\Sigma_{C}\right)+\vec{D}+N$ is recalled. Backward vector meson production, that should be background free in a collider, can possibly be measured and be used also as an alternative method of producing neutron beams. Simple estimations of cross sections are presented on the basis of existing literature. When possible, model independent statements on polarization effects are highlighted.

PACS: 13.85.-t; 14.40.-n; 14.40.Lb

Introduction

Among the wide possibilities opened by the future availability of the beams from the NICA collider and the operation of the large acceptance SPD detector, we focus here on two issues: charm production (hidden and open) and backward vector meson production. The study of such channels will take full advantage of the possibility of accelerating polarized $p, d$ beams (as well as heavier ions) in a kinematical region where data are scarce on cross sections and polarization effects are mostly unmeasured. New, precise data will be extremely useful for the understanding of the mechanism of charm creation and of hadronic matter dynamics in the non-perturbative region of QCD. In general, threshold meson production channels in $N N$-collisions, $p+p \rightarrow p+p+\omega(\phi), p+p \rightarrow \Lambda\left(\Sigma^{0}\right)+K^{+}+p$, and $p+p \rightarrow p+p+\eta\left(\eta^{\prime}\right)$, give deeper insight in the reaction mechanisms as it is shown by the experimental programs at different proton accelerators as SATURNE and COSY.

In this respect, $J / \psi$ production has a specific interest: the production and the propagation of charm in ion-ion collisions have been considered as one of the most promising probe of quark-gluon plasma (QGP) [110], but in order to state the evidence of a clear signal, it is necessary to analyze in detail all possible mechanisms for $J / \psi$ production in ion-ion collisions, and also all other processes which are responsible for the dissociation of the produced $J / \psi$ meson. The study of charmonium (hidden strangeness) and $D\left(D^{*}\right)$ mesons (open charm) are equally important.

\subsection{Charm production}

The elementary $p p$ cross section are collected and illustrated in Ref. [111].

In the energy region that can be investigated with the NICA-SPD facility, $3.4 \leq$ $\sqrt{s}[\mathrm{GeV}] \leq 27[112]$ for $p p$ collisions, the total $p p$ cross section is relatively constant around $40 \mathrm{mb}$, whereas the elastic cross section decreases due to the opening of different inelastic channels as the energy increases ${ }^{2}$. The order of magnitude of the inelastic cross section can therefore be sizable, reaching $30 \mathrm{mb}$ at the highest energies. Among these inelastic cross sections, the channels $p+p \rightarrow p+p+J / \Psi$ and $p+p \rightarrow p+\Lambda_{C}\left(\Sigma_{C}\right)+D$

\footnotetext{
${ }^{1}$ This section is written by E. Tomasi-Gustafsson; E-mail: egle.tomasi@cea.fr.

${ }^{2}$ Fundaments of elastic $p p$ scattering up to LHC energies have been recently reviewed in Ref. [113] and references therein.
} 
open around $\sqrt{s_{T h r}} \sim 5 \mathrm{GeV}$ and they are expected to grow up to several $\mu b$ in the considered energy range.

The production mechanisms for charmonium (hidden strangeness) and $D\left(D^{*}\right)$ mesons (open charm) in nucleon-nucleon collision are not yet understood. The question is how charm quarks - that are not preexisting in the nucleon as valence quarks - are formed and how they hadronize. To interpret the production and the propagation of charm in heavy ion collision as a probe of quark-gluon plasma (QGP), it is necessary to have a solid theoretical background based on the understanding of elementary processes.

Experimental data and theoretical studies of $J / \psi$ production in different processes and of its decays exist: for a review, see [114] and for a most recent data collection [115]. As a result of high statistics and high resolution experiments a large information on the properties of the $J / \psi$ meson have been collected, on the production processes and on its numerous decays. From a theoretical point of view, the interpretation of the data, in particular in confinement regime, is very controversial. As an example, the $c$-quark mass is too large, if compared to predictions from chiral symmetry, but for theories based on expansion of heavy quark mass (Heavy Quark Effective Theory), this mass is too small [116].

In the threshold region, the final particles are produced in $S$-state and the spin structure of the matrix element is largely simplified. Simple considerations indicate that this region is quite wide: the effective proton size, which is responsible for charm creation, has to be small, $r_{c} \simeq 1 / m_{c} \simeq 0.13 \mathrm{fm}$, where $m_{c}$ is the $c$-quark mass, selecting small impact parameters [117]. The $S$-wave picture can therefore be applied for $q \leq m_{c}$, where $q$ is the norm of the $J / \psi$ - three-momentum in the reaction center of mass (CMS). The momenta of the produced particles are small, but the mechanisms for the production of charmed quarks must involve large scales. In Ref. [48], the near-threshold $J / \psi-$ production in nucleon-nucleon collisions was analyzed in the framework of a general model independent formalism, which can be applied to any reaction $N+N \rightarrow N+N+V^{0}$, where $V^{0}=\omega, \phi$, or $J / \psi$. Such reactions show large isotopic effects: a large difference for $p p$ - and $p n$-collisions, which is due to the different spin structure of the corresponding matrix elements.

In Ref. [48] an estimation of $J / \Psi$ production was suggested from the comparison of the cross sections for the $\phi$ and $J / \psi$ production in $p p$ collisions. The same approach, namely $\pi$ exchange in $N+N \rightarrow N+N+V^{0}$ and $\rho$ exchange for the sub process $\pi+N \rightarrow N+V^{0}$, with $V^{0}=\phi$ or $J / \psi$ was considered. For the same value of the energy excess, $Q=\sqrt{s}-2 m-m_{V}$, taking into account the different phase space volumes, coupling constants for the decay $V \rightarrow \pi \rho$, monopole-like phenomenological form factor for the vertex $\pi^{*} \rho^{*} V$, with virtual $\pi$ and $\rho$, one finds the following simple parametrization for the cross section, holding in the near threshold region only:

$$
\sigma[n b]=0.097(Q[\mathrm{GeV}])^{2} .
$$

In Ref. [118] a parametrization of exponential form

$$
\sigma[n b]=a e^{-b M_{J / \Psi} / \sqrt{(s)}}
$$

was suggested. The values $a=1000$ [nb], and $b=16.7 \mathrm{GeV}$ reproduce well the experimental data over threshold. 
The threshold for this reaction is $E_{t h}=12.24 \mathrm{GeV}$ which corresponds to $\sqrt{s}=2 m+$ $m_{J / \psi} \simeq 4.97 \mathrm{GeV}$. In Fig. 15 the data for $p+p \rightarrow J / \psi+p+p$ (red circles) and $p+A \rightarrow J / \psi+X$ (blue squares) are plotted from the recollections in Refs. [114] (filled symbols) and [115] (open symbols). Different symbols differentiate $J / \psi$ production in $p p$ or (extrapolated from) $p A$ collisions. The data, mostly collected at CERN, are reconstructed from the measurement using models and/or assumptions, and the compiled total cross section for $J / \Psi$ production may differ up to a factor of two. For example, the original reference for the measurement from Protvino at $\sqrt{s}=11.5 \mathrm{GeV}$ [119] gives $\sigma\left(p p \rightarrow\left(J / P s i \rightarrow \mu+\mu^{-}\right)+X\right)=9.5 \pm 2.5 \mathrm{nb}$, whereas the same experimental point is referenced as $\sigma=11 \pm 3 \mathrm{nb}$, in Ref. [114] and $\sigma=20 \pm 5.2 \mathrm{nb}$, in Ref. [115]. The cross section from Ref. [48] is also plotted in Fig. 15 (solid line).

Taking the value of luminosity $\mathcal{L}=10^{30} \mathrm{~cm}^{-2} \mathrm{~s}^{-1}$, one expects 3 counts/hour for such a process with a cross section of the order of $1 \mathrm{nb}$. This number is not corrected for the detector efficiency and reconstruction with identification, for example, in a missing mass. The reconstruction of $J / \Psi$ through its decay into a lepton pair, that is the preferred mode, requires two additional orders of magnitude as the branching ratio is $(\simeq 5.9 \pm 0.5) 10^{-2}$.

Note also that in the framework of the considered model, one can find a large isotopic effect, due to the different spin structure of the matrix element at threshold:

$$
\frac{\sigma(n p \rightarrow n p J / \psi)}{\sigma(p p \rightarrow p p J / \psi)}=5,
$$

which would require a correction of the experimental data on $p A$ reaction, where equal $n p$ and $p p$ cross sections are usually assumed for the extraction of the elementary cross section in $p p$ collisions. From Ref. [48] one also learns that only one polarization observable, the $J / \psi$-polarization, is identical for $p p$ and $p n$ collisions: the $J / \psi$ meson is transversely polarized - even in collisions of unpolarized nucleons. The experimental determination of the ratio of the total cross sections for $n p$ and $p p$ collisions gives important information for the identification of the reaction mechanism.

The possibility of presence of intrinsic charm as a higher order component of the development of the Fock expansion for the proton state has been discussed in Ref. [120]. Near threshold, all partons must transfer their energy to the charm quarks, within a time $t \sim 1 / m_{c}$, thus selecting short range correlations between the valence quarks. Most interesting is the deuteron case, where all six quarks must be involved coherently, giving access to the hidden color part of the deuteron wave function.

\subsection{Open charm production}

Open charm production, $N+N \rightarrow N+\bar{D}+\Lambda_{C}\left(\Sigma_{C}\right)$ gives information on scattering lengths, effective radius, hadronic form factors, and coupling constants and is also related to the dynamics of charm creation in $N N, N A, A A *$ collisions. Some predictions can be done from an analogy with strangeness production, relying on the equivalence of $\mathrm{SU}(3)$ and SU(4) symmetries, that is however, not totally reliable. Existing information and estimation indicate that near threshold cross section can be of the order of microbarns. The threshold cross section, normalized at the lowest existing value is plotted in Fig. 16, where the insert highlights the threshold region. A dedicated simulation should be done 


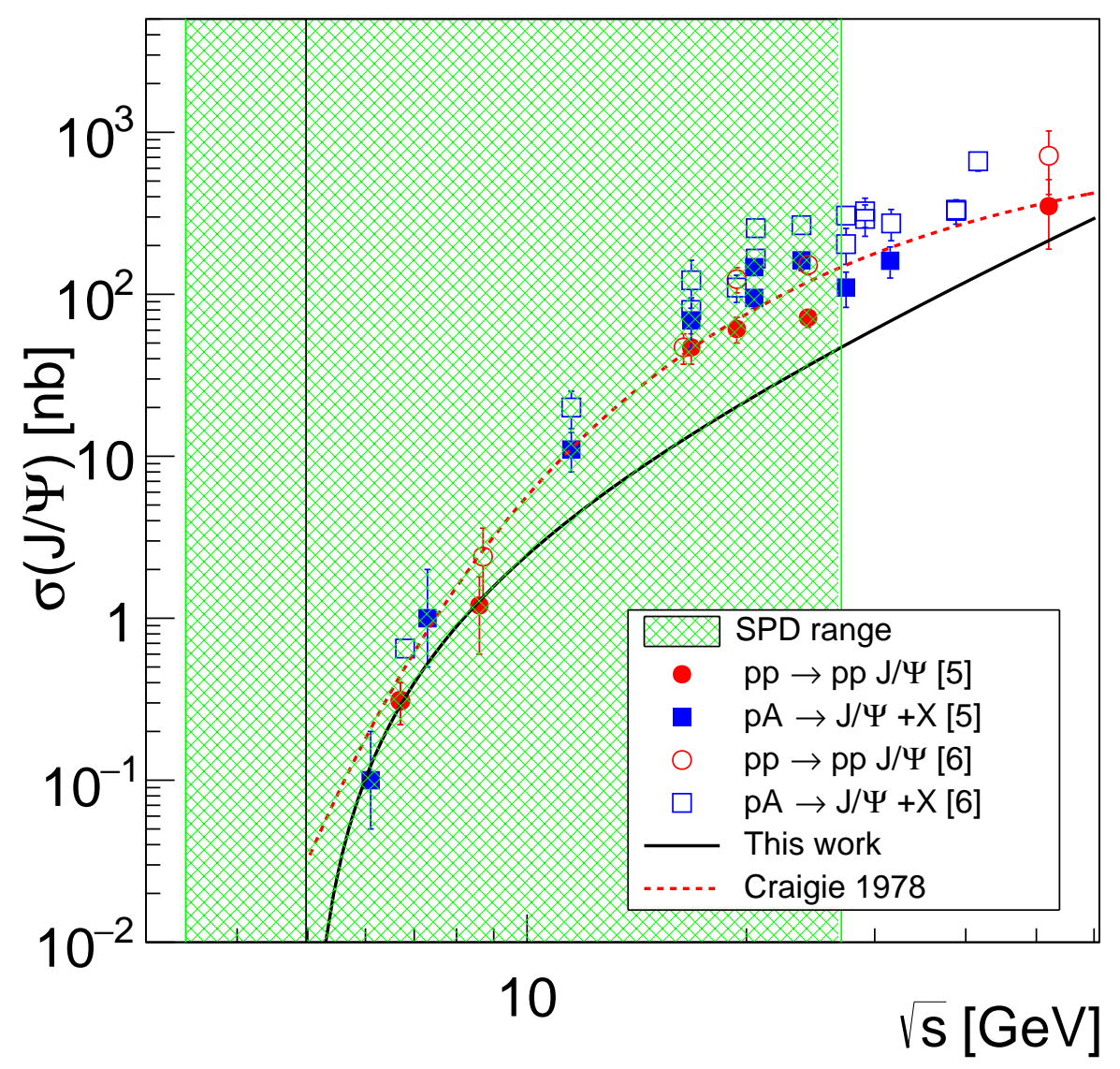

Fig. 15. Experimental data on $J \psi$ production in $p p$ (red circles) and $p A$ (blue squares) reactions, from the recollections in Refs. [114] (filled symbols) and [115] (open symbols). The solid line is the calculation from Ref. [48]. The plot is drawn from the $J / \psi$ production threshold (black line). The green filled region represents the range that can be investigated with NICA-SPD. 
to evaluate the counting rates, as the charmed particles should be reconstructed from the most suitable decay channels. The spin and isospin structure of the matrix element for the reactions $N+N \rightarrow \Lambda_{C}\left(\Sigma_{C}\right)+\bar{D}+N$ was derived for open charm in Ref. [122]. Detailed estimation of cross sections and the expressions of the polarization observables can be found there.

The charm production near threshold cross section follows the behaviour:

$$
\sigma[\mu b]=0.03(Q[\mathrm{GeV}])^{2}
$$

that can be useful for simulation purposes. It is plotted in Fig. 16 over a collection of data from Ref. [121] reanalyzed from several experiments on charm production in $p p$ and $p A$ collisions at different facilities.

We stress that these are difficult measurements, with low counting rates, but that even setting upper limits will be important, as no data at all are present in the threshold region.

\subsection{Backward meson production}

Larger counting rates are expected for light meson productions, since cross sections are of the order of mb. The $\rho^{0}$ meson production in elementary collisions and on nuclei has been discussed for example in Ref. [123] and references therein. The $\rho^{0}$ inclusive cross section has been measured at different accelerators since the 70s, mostly at CERN [124], and more recently by the HADES collaboration [125]. In Ref. [126], the inclusive cross section for $\rho$ production in $p p$ collision is calculated in frame of a generalized vector meson dominance model, and the existing data up to $\sqrt{s}=65 \mathrm{GeV}$ are fairly reproduced and compared to other models. In Ref. [127] the following parametrization was suggested

$$
\sigma\left(p p \rightarrow \rho^{0} X\right)=(0.38 \pm 0.02) \ln ^{2} s-(2.1 \pm 0.4) \text {. }
$$

This parametrization is shown together with the data for the inclusive cross section of $p+p \rightarrow \rho+X$ are in Fig. 17. One can see that it is of the order of the mb from the near threshold region, and therefore measurable at SPD already in the first phase of the experiment.

In Ref. [128] a specific kinematics, the backward light meson production in $p p$ or $p A$ collisions, was discussed in similarity with the 'quasi real electron method', where a hard photon is produced on the collision of electrons on any target [129]. Two important characteristics have been proved for the electron case: -the collinear emission probability has a logarithmic enhancement - the cross section can be factorized in a term related to the probability of the meson emission with a given energy at a given angle from the beam particle, and a term related to the interaction of the beam remnant after emission on the target.

The cross sections for the reactions of interest are:

$$
\begin{aligned}
d \sigma^{p T \rightarrow h_{+} X}(s, x) & =\sigma^{n T \rightarrow X}(\bar{x} s) d W_{h_{+}}(x), \\
d \sigma^{p T \rightarrow h_{0} X}(s, x) & =\sigma^{p T \rightarrow X}(\bar{x} s) d W_{h_{0}}(x),
\end{aligned}
$$




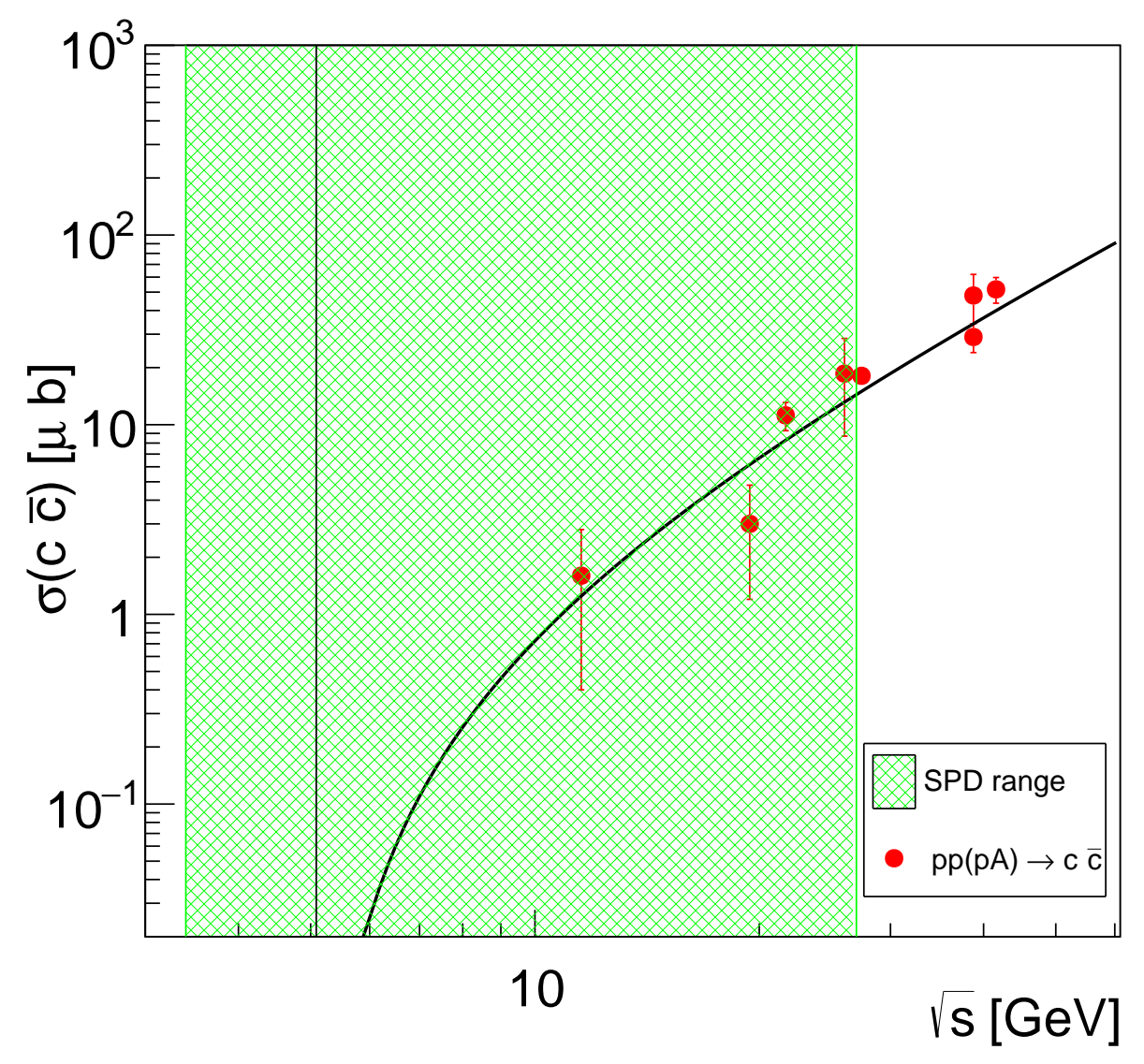

Fig. 16. Total charm production in $p p$ and $p A$ collisions. Data are from Ref. [121]. The line is a threshold parametrization (see text). 


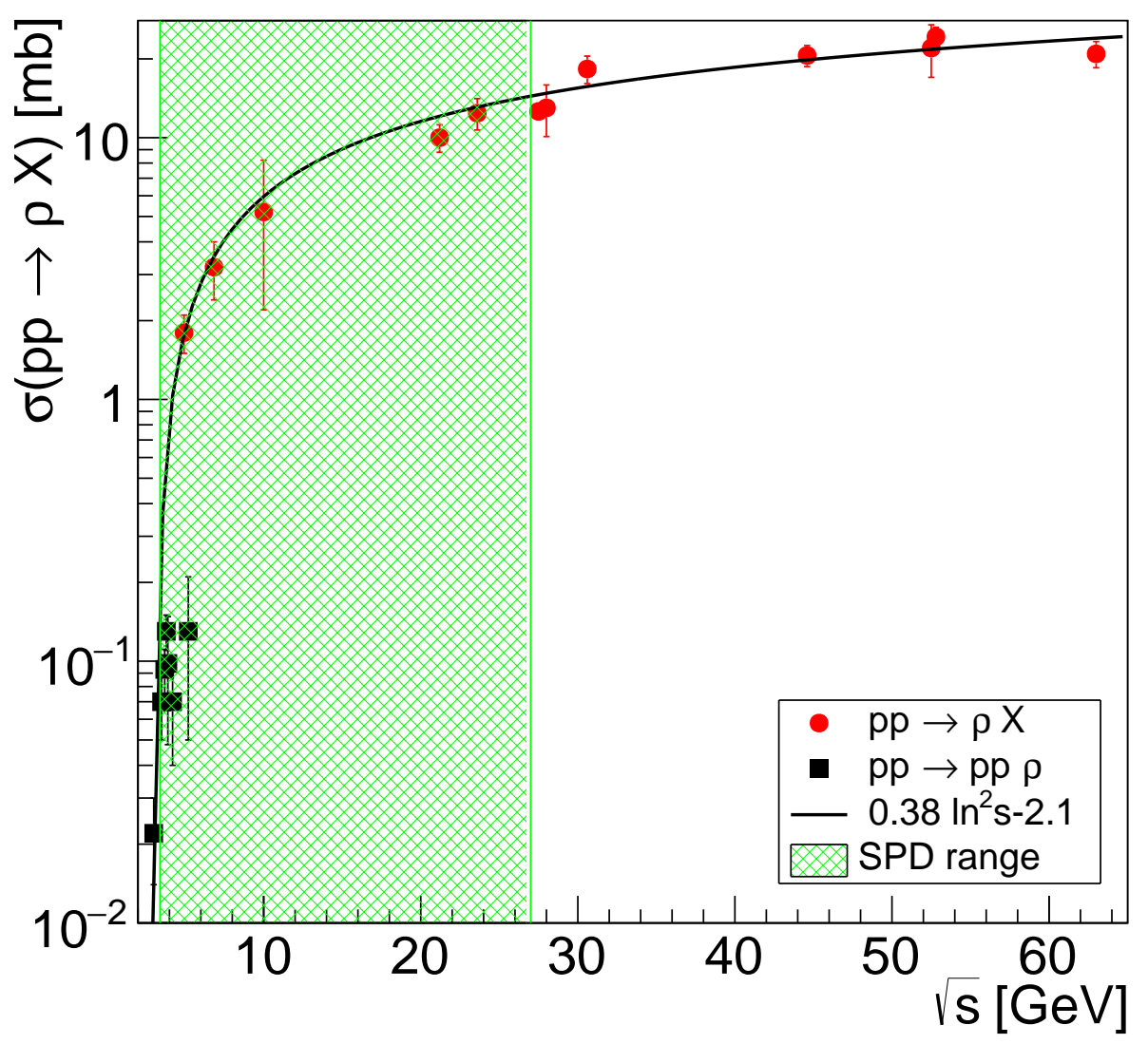

Fig. 17. $\rho$ production in $p p$ and $p A$ collisions. The red circles (black squares) are for inclusive (exclusive) $\rho$ production in different experiments. The line is the parametrization from Ref. [127]). The shaded region represents the SPD energy range. 


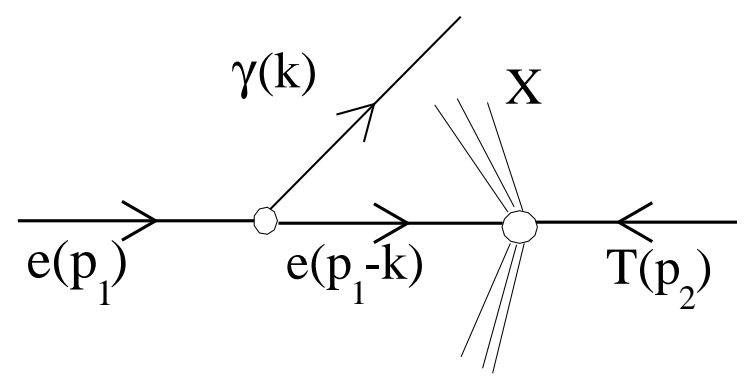

Fig. 18. Feynman diagram for collinear hard photon emission in $e T$ reactions ( $\mathrm{T}$ stands for any target). The hadron equivalent is obtained by replacing the photon by a $\rho$-meson and the electron by a proton.

where $h$ is a hadron. The quantity $d W_{\rho}(x)$ can be inferred using the QED result:

$$
\begin{aligned}
& \frac{d W_{\rho^{i}}(x)}{d x}= \frac{g^{2}}{4 \pi^{2}} \frac{1}{x} \sqrt{1-\frac{m_{\rho}^{2}}{x^{2} E^{2}}} \\
& {\left[\left(1-x+\frac{1}{2} x^{2}\right) L-(1-x)\right], } \\
& 1>x=\frac{E_{\rho}}{E}>\frac{m_{\rho}}{E}, L=\ln \left(1+\frac{E^{2} \theta_{0}^{2}}{M^{2}}\right), \rho^{i}=\rho^{+}, \rho^{-}, \rho^{0},
\end{aligned}
$$

where $M, m_{\rho}, E$, and $E_{\rho}$ are the masses and the energies of the initial proton and the emitted $\rho$-meson in the Laboratory system.

The integrated quantities $W_{h}, h=\rho, \pi$ can, in general, exceed unity, violating unitarity. To restore unitarity, we have to take into account virtual corrections: the vertex for the emission of a single pion (charged or neutral) from the proton has to include 'radiative corrections', which account for emission and absorption of any number of virtual pions. For this aim we use the known expression for the probability of emission of $n$ "soft" photons in processes of charged particles hard interaction, i.e., the Poisson formula for emission of $n$ soft photons $W^{n}=\left(a^{n} / n !\right) e^{-a}$ (where $a$ is the probability of emission of a single soft photon) [130].

The probability of emission of 'soft' neutral pions follows a Poisson distribution, which is not the case for the emission of charged pions. Fortunately, in our case, it is sufficient to consider the emission of one charged pion at lowest order (the process of one charged pion emission) plus any number of real and virtual pions with total charge zero. In such a configuration, this vertex has the form of the product of the Born probability of emission of a single pion multiplied by the Poisson-like factor:

$$
P_{\pi, \rho}=e^{-W_{\pi, \rho}}
$$

which takes into account virtual corrections. 


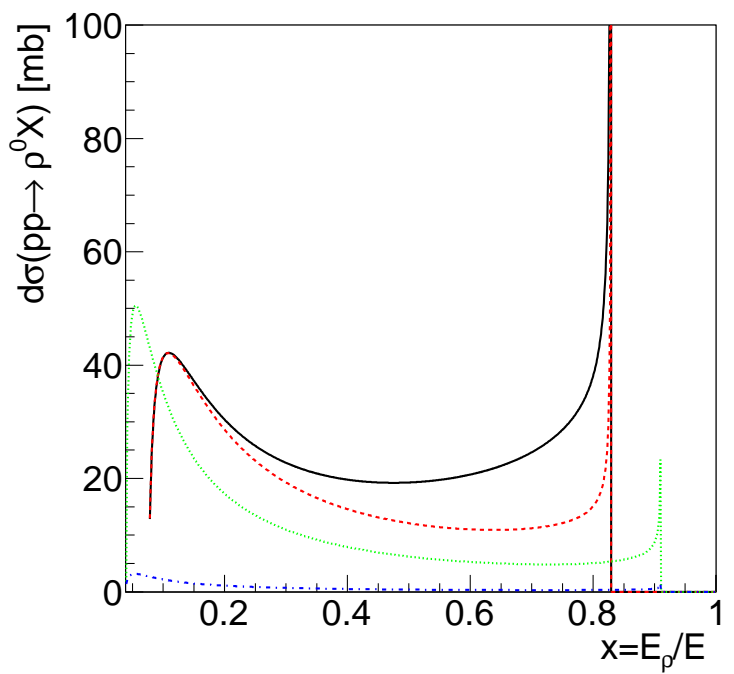

Fig. 19. Cross section $d \sigma\left(p, \bar{p} \rightarrow \rho^{0} X\right)$ as function of the $\rho$ energy fraction for two values of the incident energy and of the $\rho$ emission angle: $E=10 \mathrm{GeV}$ and $\theta_{0}=10^{\circ}$ (black, solid line), $E=10 \mathrm{GeV}$ and $\theta_{0}=20^{\circ}$ (red, dashed line), $E=20 \mathrm{GeV}$ and $\theta_{0}=10^{\circ}$ (green, dotted line), $E=20 \mathrm{GeV}$ and $\theta_{0}=20^{\circ}$ (blue, dash-dotted line).

The final result is obtained using the replacement:

$$
\sigma(s) \rightarrow \sigma(s) \times \mathcal{R}_{\pi}, \mathcal{R}_{\pi}=P_{\pi} \sum_{k=0}^{k=n} \frac{W_{\pi}^{k}}{k !}
$$

where $\mathcal{R}_{\pi}$ is the renormalization factor in order to account for the emission of $n$ real soft neutral pions escaping the detection.

Concerning the production of two charged pions, accompanied by a final state $X$, we can write:

$$
d \sigma^{p \bar{p} \rightarrow \rho^{0} X}=2 \frac{d W_{\rho}(x)}{d x} \sigma^{p \bar{p} \rightarrow X}(\bar{x} s) \times P_{\rho},
$$

where the factor of two takes into account two kinematical situations, corresponding to the emission along each of the initial particles and $P_{\rho}$ is the survival factor (32) which takes into account virtual radiative corrections. The cross section is shown in Fig. 19 as a function of the $\rho$ energy fraction, for two values of the incident energy and of the emission angle. The $x$ dependence shows a characteristic peak at $x=x_{\max }$ that has the same nature as for the QED process $e^{+}+e^{-} \rightarrow \mu^{+}+\mu^{-}+\gamma$. As explained in Ref. [131], it is a threshold effect, corresponding to the creation of a muon pair, where $x_{\max }=1-4 M_{\mu}^{2} / s, M_{\mu}$ is the muon mass.

The prediction of the model for backward $\rho$-meson production in $p p$ collisions is shown in Fig. 20, as a black solid thick line. The red dashed line is the renormalization factor, 


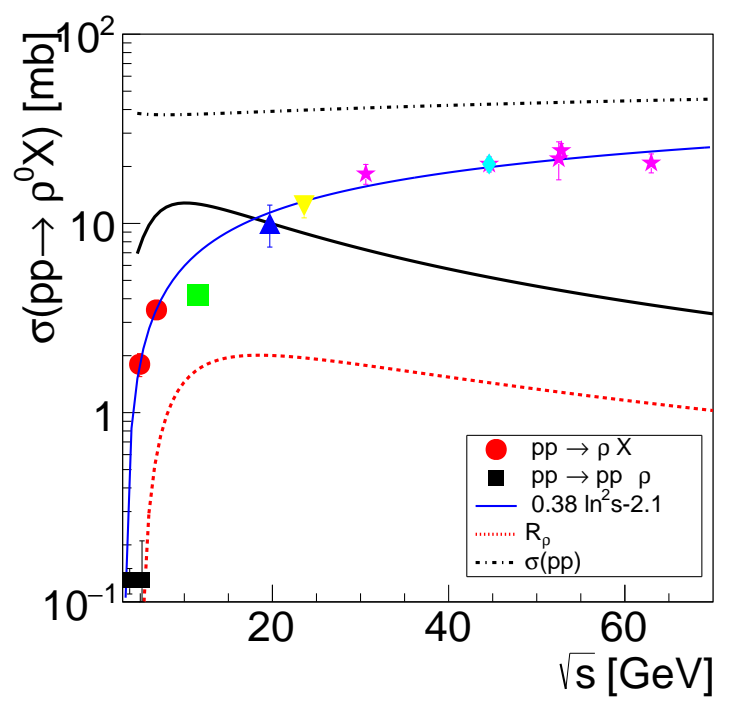

Fig. 20. Cross section for $\rho$-meson production in $p p$ collisions: inclusive (different symbols and colors from different experiments) and exclusive data from $p p \rightarrow p p \rho$ (black squares). The present calculation is shown as a black line. The red dashed line is the renormalization factor from Eq. (33). The black dash-dotted line is the total $p p$ cross section. The first red point is the inclusive measurement from Ref. [125]. The blue line is the parametrization from Ref. [127].

from Eq. (33), integrated over $x$. The total $p p$ cross section is the black dash-dotted line, of the order of $40 \mathrm{mb}$, and it is quite flat in all the considered energy region. The blue line is the parametrization from Ref. [127]) of the inclusive $\rho$ cross section. The available data are also shown, as different symbols and colors for inclusive measurements and as black squares for exclusive $\rho$ production. Backward production can be of the order of several mb, therefore accessible at NICA-SPD also with the initial lower luminosity.

An original application is the possibility of creating neutron beams by tagging the incident proton beam with a negative meson emitted backwards. Charge exchange reaction takes place, and the beam remnant is a neutron impinging on the target beam.

\subsection{Conclusions}

The understanding of charm production (open or hidden) should unify the different steps: parton-level hard process with production of $c \bar{c}$ pairs, after hadronization of $c \bar{c}$ into $J / \psi$ or into charmed hadrons (mesons and baryons) including the final state interaction of the produced charmed hadrons with other particles. The relatively large transferred momenta involved in most processes of $J / \psi$ production in hadron-hadron collisions allow to treat the first step in framework of perturbative QCD. But the applicability of QCD is not so straightforward for the description of the $c$-quark hadronization. In this respect, 
precise data collected in the NICA-SPD energy range will bring important information, especially if covering a wide range above threshold. Light meson as $\rho$ meson production is definitely easier to be measured. Collecting precise, systematic data should help to refine the models and of great interest also for the collision on heavy targets. Backward kinematics could constitute an original contribution to the field, offering an alternative possibility to produce neutron beams. 


\section{Exclusive hard processes with deuteron at NICA $^{1}$}

We argue that reaction $p^{2} H \rightarrow p p n$ at large momentum transfer to one of the nucleons of the deuteron provides a sensitive probe of space time evolution of hard $p N$ scattering. The same process in a different kinematics allow to study short range correlations in the deuteron. Use of the polarized deuteron beams would provide a unique opportunity to separate S- and D-wave contributions to the high momentum component of the deuteron wave function. A possibility to look for nonnucleonic components of the short range correlations is also outlined.

PACS number(s) 13.75.Cs, 25.10.+s, 25.40Ep

Our understanding of the dynamics of NN interactions at the energy range of $\sqrt{s} \sim 5 \div$ $20 \mathrm{GeV}$ is still rather limited. In particular, it is not clear yet where transition occurs from nonperturbative to perturbative dynamics in few body processes with a large momentum transfer $(-t)$. This includes even the most basic process of the large $-t$ elastic nucleon - nucleon scattering at large $-t$. Among the puzzles are large spin effects in large angle scattering of polarized protons [38] and a complicated energy dependence of the nuclear transparency in large angle scattering of incident protons off the protons embedded in nuclei [44]. Also the recent observations of two nucleon short range / high momentum correlations in nuclei mostly in electron - nucleus scattering (see review in $[132,133]$ ) require confirmation and testing universality of the SRCs using other projectiles - protons, photons, etc.

Questions involved in studies of the short-range / high momentum nuclear structure and understanding microscopic nucleon structure and dynamics of large momentum transfer processes are delicately intertwined: understanding of hard dynamics of two body processes is also necessary for precision studies of the short range nuclear structure.

Several strategies are possible to address these questions. Here we will concentrate on reactions with the deuteron since the nonrelativistic deuteron wave function is well known and hence the measurements could be matched to detailed theoretical calculations. Also, the use of the deuteron allows to choose special kinematic domains where $p^{2} H$ scattering is sensitive to the short-range nuclear correlations. The collider kinematics presents a number of advantages as all particles in the reactions in question have large momenta and hence can be easily detected.

7.1. Probing dynamics of nucleon - nucleon interaction in proton - deuteron quasielastic scattering

The simplest reaction which would be interesting to study is the process $p^{2} H \rightarrow p p n$ where one of the nucleons has small transverse momentum and two others have approximately back to back large transverse momenta $[134,135]$.

In the impulse approximation this process corresponds to elastic scattering of the projectile proton off a quasifree nucleon of the target. There exist however kinematical conditions where the dominant contributions are due to soft rescatterings of the initial

\footnotetext{
${ }^{1}$ This section is written by M. Strikman; E-mail: mxs43@psu.edu
} 
and final nucleons, which accompany the hard $p p(p n)$ reaction. The eikonal approximation, which accounts for relativistic kinematics as dictated by the Feynman diagrams, reveals the important role played by the initial and final state interactions in the angular and momentum dependences of the differential cross section in well defined kinematics. The condition for the applicability of the generalized eikonal approximation [136] is that the c.m. scattering angle and invariant mass of the two nucleon system are large enough so that $-t,-u \geq 2 \mathrm{GeV}^{2}$.

It was suggested in $[5,6]$ that nucleons in the elementary reaction interact in small size configurations with a small cross section - so called color transparency phenomenon. This effect is suppressed by the space - time evolution of nucleon wave packets $[137,138]$. However effect of evolution is very small for the deuteron where typical distances between nucleons in the rescattering amplitude are is $\leq 1.5 \mathrm{fm}$. Hence the discussed process allows to measure the wave packet size of a nucleon practically right in the interaction point.

It was pointed out that the hard dynamics in $p p$ and $p n$ elastic scattering may be rather different [139]. Hence it would be instructive to compare the channels where $p p$ and $p n$ are produced with large $p_{t}$.

Experiments with polarized beams would greatly add to this program: a) study of the dependence of the cross section on the deuteron polarization allows to improve separation of kinematic domains where impulse approximation, double and triple scattering dominate, while the studies $\vec{p} \vec{d} \rightarrow p N N$ processes will allow both to study spin structure of $p p$ and $p n$ elastic scattering at large $t$ (the later is practically not known). Also, it would be possible to find out whether the A. D. Krisch effect [38] (a strong difference between the cross sections of elastic scattering of protons with parallel and antiparallel spins) involves collisions of protons in configurations with sizes depending on the spin orientation.

It would be possible also to study effects of coherence in the channels where exchange by gluons in t-channel is not possible, for example $p d \rightarrow \Delta N N$. In particular, it would be possible to test the effect of chiral transparency suggested in [140] - suppression of the pion field in the nucleons experiencing large $-t$ scattering.

\subsection{Probing microscopic deuteron structure}

As emphasized in the introduction, the dominant source of the SRC in nuclei are proton - neutron correlations with the same quantum numbers as the deuteron and with high momentum tail similar to that in the deuteron. Hence the deuteron serves as a kind of the hydrogen atom of the SRC physics. Only after it would be tested experimentally that approximations currently used for the description of the $p^{2} H$ reaction work well, it would be possible to perform high precision studies of SRC in heavier nuclei.

It was demonstrated in Ref. [134,135] that under specific kinematical conditions (in particular low transverse momenta of a slow nucleons in the deuteron rest frame)) the effect of initial and final state interactions can be accounted for by rescaling the cross section calculated within the plane wave impulse approximation. In this kinematics it would be possible to check universality of the wave function - in particular its independence on the momentum transfer in the elementary reaction. Such factorization is expected to break down at sufficiently large $-t$ and $-u$ where scattering involves interaction of 
nucleons in the small size configurations (the color transparency regime) since the small size configurations are suppressed in bound nucleons with suppression growing with the nucleon off shellness [138].

Studies of the nonnucleonic configurations in the deuteron as well as relativistic effects. are of separate interest. One option is a search for non-nucleonic degrees of freedom like 6 quark, two $\Delta$ isobars via production reaction $p^{2} H \rightarrow \Delta^{++}+p+\Delta^{-}$with $\Delta^{++}$and proton back to back and $\Delta^{-}$in the deuteron fragmentation region for the light cone fraction $\alpha_{\Delta}=2 p_{\Delta}^{-} / p_{d}^{-} \geq 1$ (slow in the deuteron rest frame; proton momentum is along $z$ direction).

In a long run, when polarized deuteron beams would become available, it would be possible to to separate contribution of the S- and D- wave to the SRCs and compare different relativistic models of the deuteron - light -cone vs virtual nucleon approximations. The difference between two predictions is large already for the nucleon momenta $\sim 300 \mathrm{MeV} / \mathrm{c}$ in the deuteron rest frame. 


\section{Scaling behaviour of exclusive reactions with lightest nuclei and spin observables ${ }^{1}$}

Differential cross sections of various binary reactions with the lightest nuclei at large fixed scattering angles are in qualitative agreement with the $s$ - power-law dependence dictated by the constituent counting rules. We propose to measure differential cross section and deuteron analyzing powers of the $d p$ - elastic scattering at the SPD NICA to search for transition region from meson-baryon to quark-gluon degrees of freedom in the deuteron structure.

PACS: 21.45.+v; 24.70. $+\mathrm{s} ; 25.10 .+\mathrm{s}$

The structure of the lightest nuclei at short distances $r_{N N}<0.5 \mathrm{fm}$ or high relative momenta $\left(q>\hbar / r_{N N} \sim 0.4 \mathrm{GeV} / c\right.$ constitues a fundamental problem in nuclear physics. One of the most important questions is related to search for onset of transiton region from meson-baryon to quark-gluon picture on nuclei. A definite signature for transition to the valence quark region is given the constituent counting rules (CCR) [141,142]. According the dimensional scaling the differential cross section of a binary reaction at enough high incident energy can be parametized as $d \sigma / d t \sim s^{-(n-2)} f(t / s)$, where $n$ is the sum of costituent quarks in all participants, $s$ and $t$ are Mandelstam variables. Many hard processes with free hadrons are consistent with CCR at energies of several GeV. The CCR propetrties of the reactions with the lightest nuclei were observed in photodisintegration of the deuteron $\gamma d \rightarrow p n$ at $E_{\gamma}=1-4 \mathrm{GeV}$ and ${ }^{3} \mathrm{He}$ nucleus ${ }^{3} \mathrm{He}(\gamma, p p) n, \gamma^{3} \mathrm{He} \rightarrow d p$. Earlier data on the reaction $d d \rightarrow^{3} H p, \quad d d \rightarrow^{3} H e n[143]$ and $p d \rightarrow p d$, as was show in Ref. [144] also follow CCR behavior $s^{-22}$ and $s^{-16}$, respectively, at surprising low energies, $0.5 \mathrm{GeV}$. Recently the CCR behaviour of the reaction $p d \rightarrow p d$ was observed in $[145,146]$ at higher energies. On the other hand, the reaction with pion production $p p \rightarrow d \pi^{+}$ does not follow CCR rule demostrating the differential cross section $\sim s^{-9}$ instead of $s^{-12}$. One possible way to explain this is a partial restortaion of chiral symmetry at enough high excitaion energy [147]. However, systematic study of these properties of the reactions with lightest nuclei are absent. So, important to know whether reaction $p n \rightarrow d \rho^{0}$ follows the CCR behavior and at what minimal energy there is the CCR onset. Assuming the model of the vector meson dominance and taking into account the observed CCR behavior of the $\gamma d \rightarrow p n$ reaction, one may expect the $\sim s^{-12}$ dependence of the cross section of the reaction $p n \rightarrow d \rho^{0}$. Furthermore, possible relation between CCR behavior of the upolarized cross section and spin observables of the same reaction are practically not known. The SPD NICA facility provides a good opportunity for this study using polarized beams in $p p, d d$ and $p d$ collisions.

The results on the polarization observables for deuteron induced reactions at large transverse momenta are controversial. Results on the tensor polarization $t_{20}$ for the ed- elastic scattering [148] obtained at JLab can be reproduced quite well at $Q^{2} \leq 1.7$ $(\mathrm{GeV} / \mathrm{c})^{2}$ by the covariant relativistic model [149] without contribution of non-nucleonic degrees of freedom. The perturbative QCD (pQCD) predictions [150] are not reliable for these momentum transfers. On the other hand, while the electromagnetic form factors for the deuteron in the soft-wall AdS/QCD model [151] are well in agreement with the

\footnotetext{
${ }^{1}$ This section is written by V.P. Ladygin (E-mail: vladygin@jinr.ru) and Yu.N. Uzikov
} 
experimental data and the form factors display correct $1 / Q^{10}$ power scaling for large $Q^{2}$ which is consistent with the CCR, the tensor analyzing power $t_{20}[148]$ demonstrates the discrepancy with the soft-wall AdS/QCD predictions [152].

The cross section of high energy two-body photodisintegration of the deuteron, $\gamma d \rightarrow$ $p n$, at large angles in the cms $[153,154]$ has shown the scaling behavior up to $5.5 \mathrm{GeV}$ predicted by CCR $[141,142]$. Recent measurements of the proton polarization [155] at energies up to $2.4 \mathrm{GeV}$ are also consistent with the pQCD hadron helicity conservation prediction [156], but not the polarization transfers obtained in the same experiment.
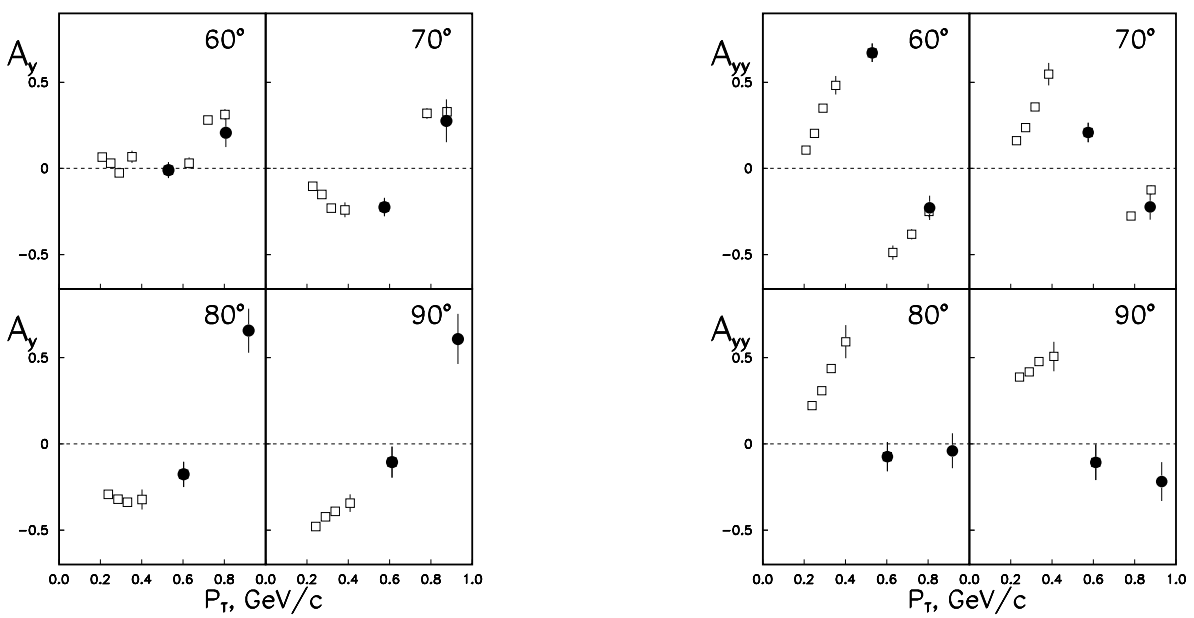

Fig. 21. Vector $A_{y}$ (left panel) and tensor $A_{y y}$ (right panel) analyzing powers in $d p$ - elastic scattering obtained at the fixed angles in the cms: $60^{\circ}, 70^{\circ}, 80^{\circ}$ and $90^{\circ}$.

The dependencies of the vector $A_{y}$ and tensor $A_{y y}$ analyzing powers in $d p$ - elastic scattering obtained at $60^{\circ}, 70^{\circ}, 80^{\circ}$ and $90^{\circ}$ in cms versus transverse momentum $p_{T}$ are shown in Fig.21 in the left and right panels, respectively. The open points are the world data obtained at RIKEN, Saclay and ANL, while the black ones represent the results obtained at Nuclotron $[157,158]$ The analyzing powers demonstrate the sign changes at $p_{T} \sim 650 \mathrm{MeV} / c$ and the asymptotic behavior at large transverse momenta. Note, that negative sign of $A_{y y}$ is observed also in deuteron inclusive breakup at large $p_{T}$ [159], [160].

It would be interesting to extend the range of the measurements to larger $p_{T}$, where the manifestation of non-nucleonic degrees of freedom is expected. New precise measurements with small statistical and systematic uncertainties at the energies higher than $\sqrt{s} \geq 3.3 \mathrm{GeV}$ and at different scattering angles are required to make a conclusion about the validity of CCR $[141,142]$ in $d p$ - elastic scattering. We propose to measure also different vector and tensor analyzing powers in $d p$ - elastic scattering at SPD energies.

The tensor $A_{y y}$ and vector $A_{y}$ analyzing power in $d p$ - elastic scattering obtained at $60^{\circ}, 70^{\circ}, 80^{\circ}$ and $90^{\circ}$ in cms versus transverse momentum $p_{T}[157,158]$ demonstrates the negative and positive asymptotics, respectively. Note, that negative sign of $A_{y y}$ 
is observed also in deuteron inclusive breakup at large $p_{T}$ [159], [160]. It would be interesting to extend the range of the measurements to larger $p_{T}$, where the manifestation of non-nucleonic degrees of freedom is expected. New precise measurements with small statistical and systematic uncertainties at the energies higher than $\sqrt{s} \geq 3.3 \mathrm{GeV}$ and at different scattering angles are required to make a conclusion about the validity of CCR $[141,142]$ in $d p$ - elastic scattering. We propose to measure also different vector and tensor analyzing powers in $d p$ - elastic scattering at SPD energies.

The measurements of $d p$ - elastic scattering can be performed either with polarized deuterons and unpolarized protons, or with unpolarized deuterons and polarized deuterons. The $d p$ - elastic scattering events can be selected using cuts on the azimuthal and polar scattering angles correlations. The vector $A_{y}$ and tensor $A_{y y}$ and $A_{x x}$ analyzing powers will be measured simultaneously in the case of the vertically polarized deuteron beam. The precision on the tensor $\Delta A_{y y} \sim 0.09$ and $\Delta A_{x x} \sim 0.09$ and on the vector $\Delta A_{y} \sim 0.03$ analyzing powers can be achieved for the scattering angle $\sim 90^{\circ} \pm 5^{\circ}$ at $\sqrt{s} \sim 4.5 \mathrm{GeV}\left(p_{T} \sim 1.7 \mathrm{GeV} / c\right)$ for 30 days of the beam time at the luminosity $\mathcal{L} \approx 10^{29} \mathrm{~cm}^{-2} \cdot \mathrm{s}^{-1}$. We assume $\sim 75 \%$ of the beam polarization from the ideal values of polarization for different spin modes. The counting rate has been estimated using $d p$ elastic scattering cross section parameterization from Ref. [146]. The spin correlations can be obtained in quasi-free $d p$ - elastic scattering using $d d$ - collisions. 


\section{Multiquark correlations and exotic hadron state production ${ }^{1}$}

Multiquark correlations in the collisions of particles and nuclei at NICA energies play an important role in understanding of QCD. Processes involving multiquark degrees of freedom can shed light on various aspects from multiquark fluctons, diquarks, multiparton scattering to exotic resonance production and fulfil a broad and rich physics program at SPD experiment.

PACS: $44.25 .+\mathrm{f} ; 44.90 .+\mathrm{c}$

\subsection{Multiquark correlations and exotic state production at SPD NICA}

Multiquark correlations in the collisions of particles and nuclei play an important role in understanding of QCD. Multiquark correlation phenomena may be divided into four classes.

The first one can be related with parton distribution functions (PDFs) of the colliding nuclei. In the leading twist approximation, in the nuclear PDFs there is a contribution at large $x>1$, which is related with objects fluctuations of nuclear matter [161] known as multiquark fluctons [162-164] (see for a review, e. g., Refs. [165, 166] and references therein) or few-nucleon short-range correlations (see, e. g., Ref. $[138,167,168]$ and references therein). Fluctons are compact multinucleon states with defrosen color quark-gluon degrees of freedom, see, e. g., [162-164, 169-180].

The second class is connected with higher twist contributions of two- and/or threequark correlations in PDFs of hadrons and nuclei.

The third class is dealing with multiparton scattering subprocesses in hadronic and nuclear collisions. Multiparton scattering occurs when two or more partons from each colliding objects simultaneously scatter off each other.

The forth class phenomena can be related with production of exotic multiquark resonance states, e. g., pentaquark and tetraquark states.

Below one can briefly outline possible studies, which can shed light on the all mentioned above classes of multiquark phenomena and fulfil a broad and rich physics program at SPD experiment.

9.2. Multiquark correlations: fluctons in nuclei

Fluctuations of nuclear matter were considered in Ref. [161], which were discussed after discovering backward scattering off nuclei [181,182], would form fluctons, compact multinucleon states or in other language, short-range few nucleon correlations.

Fluctons are directly connected with cumulative hadron production in the nuclear fragmentation region $[183,184]$. The flucton approach [179], which is based on hard QCD-factorisation and EMC-ratio constraints, predicts an extra nuclear quark sea, which has rather hard momentum distribution: the extra nuclear sea $x$-slope is equal to the

\footnotetext{
${ }^{1}$ This section is written by V.T. Kim (kim_vt@pnpi.nrcki.ru), A.A. Shavrin (shavrin.andrey.cp@gmail.com) and A.V. Zelenov (zelenov_av@-pnpi.nrcki.ru)
} 
$x$-slope of the valence quarks at $x>1$. It leads to "superscaling" for cumulative hadron production at $x>1$ in the nuclear fragmentation region: the $x$-slope of all cumulative hadron distributions including "sea" ones [179,185] are the same. The superscaling phenomenon was experimentally confirmed by ITEP group [186, 187].

Nuclear fluctons consist of the nucleons compressed in distances comparable with nucleon size, so the flucton with five or six nucleons could be considered as a cold dense baryon matter since the effective nuclear density would be high as that in the core of neutron stars [132]. In such a dense nuclear medium there would be $C P$-violating effects [188].

In high- $p_{T}$ cumulative processes at the central region, other contributions should be added to the contribution of the nuclear PDFs at $x>1$, such as the contributions from the PDFs of the other colliding object and possible intranuclear rescattering effects [189, 190]. So, beyond the nuclear fragmentation region one should observe deviations from superscaling for cumulative production.

For many observables there are two popular approaches: fluctons and short-range nucleon correlations yield similar predictions, e. g., cumulative particle production, nuclear structure functions in deep inelastic processes with leptons, etc. However, there is the main difference with flucton and short-range nucleon correlation approaches: extra sea quark degrees of freedom atributted to flucton sea in nuclear PDF at $x>1$.

The hard flucton antiquark sea can manifest in massive lepton pair [191-193] and $J / \psi$ production in cumulative region $[194,195]$. SPD can study $J / \psi$ production process in $p d$ and $d d$ collisions in $x>1$, which should be highly sensitive for hard flucton antiquark sea of deutrons.

\subsection{Few-quark correlations: Diquarks}

Another type of multiquark correlations is few-quark short-range correlations: diquark and triquark states in baryons. Diquark states were discussed soon after suggesting quark model for hadrons, see for a review Refs. [196,197]. This is an important source [198-202] of large- $p_{T}$ baryon production $[200,203,204]$. Being a higher-twist the diquark contribution can describe the strong scaling violation in deep inelastic scattering off nucleons and in large- $p_{T}$ baryon production in hard nucleon collisions at SPD energies $[201,202,205]$ (Fig. 22)

Most of diquark studies were done within inclusive approach. ABCDHW Collaboration at CERN ISR have found more convincing indications [207,208] on diquark manifestations in two-particle correlations. SPD allows to study two-particle correlations between large- $p_{T}$ baryons (proton, $\Delta^{++}, \Lambda^{0}$, etc. ) and back-to-back hadrons to reveal of quark structure of proton in more detail.

Therefore, measuring by SPD experiment various two-particle correlations should reveal more detail diquark structure of proton. 


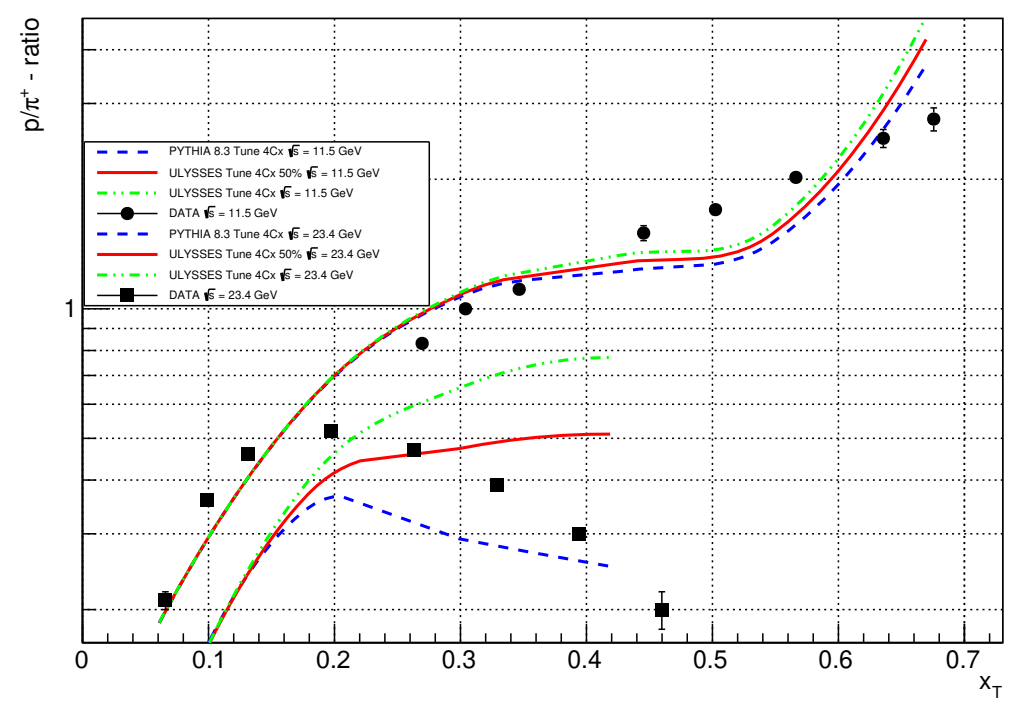

Fig. 22. $p / \pi^{+}$-ratio of inclusive invariant cross sections at $\theta_{c m s}=90^{\circ}$ in $p p$-collisions confronted with the data from IHEP, Protvino [204] $(\bullet)$ at $\sqrt{s}=11.5 \mathrm{GeV}$ and from FNAL, Batavia [203] (ם) at $\sqrt{s}=23.4 \mathrm{GeV}$. The preliminary predictions from MC event generator ULYSSES [205] with incorporated diquark subprocesses: the solid red line corresponds diquark state probablity in proton equals 0.5 , the dash-dotted green line - 1.0. Prediction by the standard PYTHIA8.3 $4 \mathrm{Cx}$ [206] without diquark subprocesses is shown by the blue dashed line.

\subsection{Multiparton scattering}

There is an important aspect of hadronic collsions, which was studied since 70s [209-216]. There are two basic approaches for description of multiparton scattering, depending on view whether it is considered as a higher twist effect or not [217-221]. In the all approaches the main object is momentum multiparton momentum-dependent distribution function, which is complicated in addition by the multiparton distribution in space (impact plane). Therefore, measuring few-particle correlation at SPD one can study multiparton scattering processes [205], which are related with 2D- and 3D- PDFs in momentum and coordinate spaces.

SPD experiment can study multiparton distribuion in proton via multiparton scattering, which yield, in particular, certain azimuthal two-particle correlations. For instance, double-parton scattering should increase two-particle correlations out of back-to-back scattering region, where single parton-parton scattering is dominant [179] yielding a ridge structure [223]. Predictions from MC event generators ULYSSES [205] with incorporated diquark subprocesses and PYTHIA8.3 for two-particle correlation function of two protons

$$
R_{12}=\sigma^{i n} \frac{\frac{E_{1} E_{2} d^{6} \sigma}{d^{3} p_{1} d^{3} p_{2}}}{\frac{E_{1} d^{3} \sigma}{d^{3} p_{1}} \frac{E_{2} d^{3} \sigma}{d^{3} p_{2}}}
$$

is shown in Fig. 23 for back-to-back region and outside it at $\sqrt{s}=23.4 \mathrm{GeV}$. 


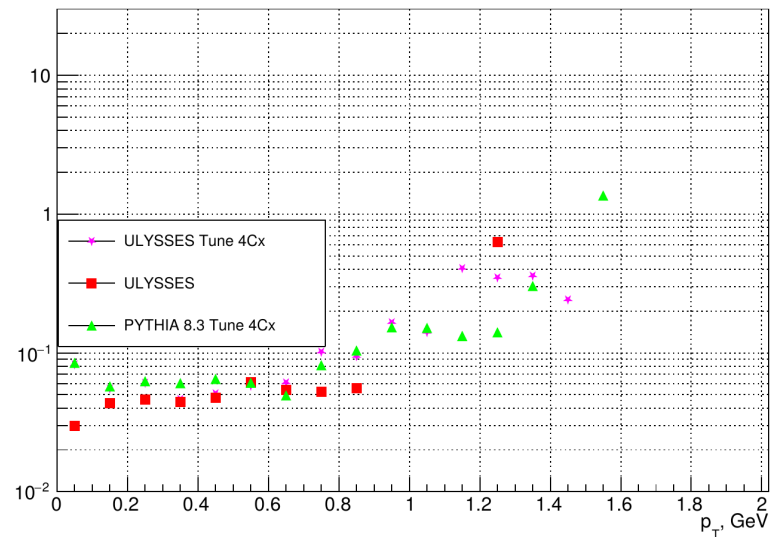

(a)

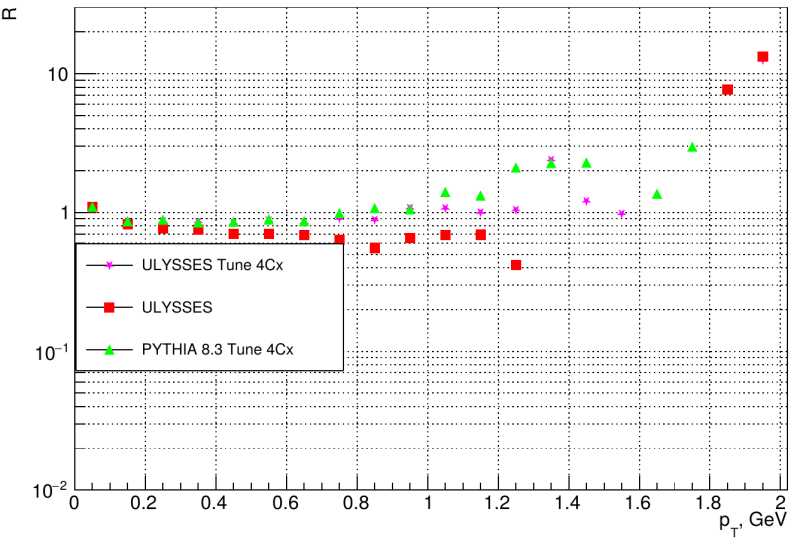

(b)

Fig. 23. Two-proton correlation function at $\theta_{c m s}=90^{\circ}$ in $p p$-collisions at $\sqrt{s}=23.4 \mathrm{GeV}$ as a function of $p_{T}\left(\approx p_{T 1} \approx p_{T 2}\right)$ for two regions of azimuthal angle difference: (a) for back-to-back region $\left(\Delta \varphi=\left|\varphi_{1}-\varphi_{2}\right| \approx \pi\right)$ and (b) out of it $(\Delta \varphi \not \approx \pi)$. The preliminary predictions from MC event generator ULYSSES [222] with incorporated diquark subprocesses in different tunes (red squares and magenta stars) and PYTHIA8.3 4Cx [206] (green triangles) without diquark subprocesses.

Two-particle correlation function for out of back-to-back azimuthal region is directly connected with double-parton scattering cross section.

Multiparton scattering can be significant for production of multiquark systems and and nuclei $[205,224]$.

\subsection{Multiquark exotic state production}

Multiparton scattering provide a unique opportunity to study production of various multiquark states and light nuclei $[205,224]$ at SPD energies. 
An excitement has been flourished when LEPS [225], DIANA [226] and CLAS [227] Collaborations observed a resonance state, which was correspondent to quantum flavour numbers of $\Theta^{+}$-pentaquark, with the narrow width as predicted in Ref. [228].

However, in a meantime there was a somewhat contradictory situation. Most of $\Theta^{+}$pentaquark observation claims were ruled out while the others [226,229-231] were still not, see for a review of the experimental situation, e. g., Ref. [232, 233]. There were also papers tried to explain why one experiments could observe the $\Theta^{+}$-pentaquark state while the others would not, see, e. g., Ref. [234]. A similar situation with the other multiquark states: H-dihyperon $[235,236]$, etc. $[237,238]$.

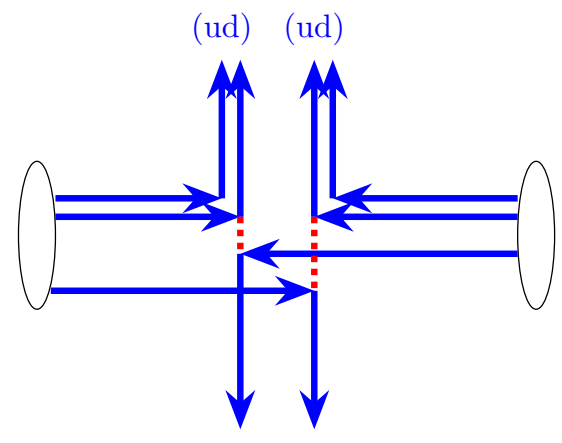

Fig. 24. Production of multiquark systems (pentaquarks,H-dihyperons, etc.) and deutrons on large angles via double diquark-quark scattering $[205,224]$ in nucleon collisions.

Multiquark state production cross section depends from its structure and production mechanism. For instance, the atomic or molecular structures lead to different mechanisms of production and to different branching ratios of the final states.

Production of multiquark systems with possible diquark structure $[222,239-242]$ on large angles can be enhanced due to double diquark-quark scattering [205, 224]. Inclusive and two-particle correlation studies with production of light nuclei and antinuclei should help establishing two production mechanism stages: multiparton scattering and fragmentation/fusion process [205,224] (Fig. 24).

So far, most of the negative pentaquark and H-dihyperon searches were performed at small production angles. Therefore, SPD experiment provides an uniqe opportunity to search such multiquark states at unexplored kinematic domain.

\subsection{Summary}

To summarise this section:

- SPD with study of the inclusive particle production and few-particle correlations at different kinematic regions in $p p$-collisions at high luminosity should reveal such quark correlations as diquark states in proton; 
- Multiparton distribution in proton can be studied via multiparton scattering, which yield certain azimuthal two-particle correlations; In particular, double-parton scattering should increase two-particle correaltions out of back-to-back scattering region;

- SPD can carry out investigations of the novel production mechanisms as double quark-diquark scattering and the other multiparton scattering subprocesses, which would lead to enhanced production of exotic multiquark resonance states and light nuclei at large angles;

- $p d$ - and $d d$-collisions at high luminosity can shed light on the difference between multiquark fluctons and short-range nucleon correlations by measuring cumulative production of antiprotons and $K^{-}$mesons. Collision processes with light nuclei, if available, should enhance sensitivity to the hard flucton antiquark sea.

Therefore, SPD experiment at NICA has a unique opportunity, from one side, to test and extend our knowledge of various aspects of multiquark correlations: from diquark substructure of proton, double-parton scattering, the cold dense baryon matter, to the exotic multiquark resonance production, and, from the other side, it has a rather high potential for discovering new exotic multiquark states and novel mechanisms of production at high energy hadronic collisions. 


\section{Study of inelastic d-d and p-d interactions for observation of neutron-proton system under strong compression ${ }^{1}$}

In this experimental proposal, we consider a possibility of registering light dibaryons at the NICA SPD facility, experimental and theoretical indications of the existence of which were previously obtained at JINR. The main attention is paid to the description of the observable effects, as well as the requirements for measuring instruments necessary for their successful detection.

PACS: 24.85.+p, 13.88. $+\mathrm{e}, 13.85 . \mathrm{Fb}$

\subsection{Introduction}

At present, there are different theoretical indications that cold nucleonic matter under strong compression should undergo phase transitions, see, e.g., [243]. Experiments at NICA MPD will be devoted to the search for signs of these transitions, in which the manifestations of the transformations of the nucleon phase into quark-gluon one, as well as other transitions, is expected to be seen in collisions of heavy nuclei. Unfortunately, traditional nuclear physics, as well as astrophysical restrictions on the equation of state for cold dense matter, do not yet give direct indications of the need to take into account such processes [244]. A similar situation has arisen in the description of small nucleon systems, which can be used as the basis for the microscopic theory of phase transitions in nuclear matter. Thus, it has long been suggested that various multibaryon states can exist in nature in the form of a configurational admixture together with ordinary nuclei [245]. At the same time, models continued to develop, suggesting that nucleons in nuclei can also retain their individuality even in very exotic high-momentum states [246]. An attempt to find experimental manifestations of the multiquark component in the wave function of atomic nuclei was undertaken in the frame of the flucton model [247,248]. In parallel, the model of short-range correlations according to which, to explain the same processes, the nucleon models, taking into account the high-momentum component of the wave function of nuclei, were also quite suitable [249].

Experiments on the NICA MPD facility should in the future answer some of the questions associated with the problem of phase transitions of nucleonic matter into other forms of nuclear substance at high densities, which can be achieved in collisions of heavy nuclei accelerated to relativistic velocities. The theory of these phenomena is extremely difficult, so that the interpretation of the obtained experimental data may turn out to be highly uncertain. In this regard, it is of particular interest to study the transitions of nucleonic matter to other states, which can occur in few-nucleon systems. Drawing an analogy with the theory of the formation of an electron-ion plasma, it can be argued that, in this respect, knowledge of the characteristics of few-nucleon systems under conditions of strong compression can contain information as indispensable as knowledge of the ionization characteristics of atoms for building a theory of ordinary plasma.

\footnotetext{
${ }^{1}$ This section is written by B.F. Kostenko, E-mail: bkostenko@jinr.ru
} 
10.2. Search for new dibaryons at the NICA SPD facility

There are a number of theoretical and experimental indications of the existence of light dibaryon excitations in 2-nucleon systems [250-252]. An analysis of the experimental data suggests the possible observation of events, the cross sections of which in a certain kinematic region are comparable to the cross sections of hard elastic nucleon-deuteron collisions. For example, in [252] a peak was observed, which is interpreted to date as a quasi-free knockout by the target deuteron of a nucleon from the incident deuteron in the reaction $\mathrm{d}+\mathrm{d} \rightarrow \mathrm{d}+\mathrm{X}$. However, its large width can hardly be explained only by the internal motion of nucleons in the deuteron being destroyed. At the same time, kinematic analysis shows [250] that scattering processes with the participation of virtual dibaryons preexisting in the incident deuteron in a virtual form before the collision can also contribute to the observed peak. In experiments [251], where the effective masses of two-nucleon systems formed after the emission of several (up to 5) pions from highly excited neutron-proton systems were measured, there are indications of the observation of narrow dibaryons, the masses of which can be described by a simple formula [250] $M_{n}=M_{d}+10.08 n \mathrm{MeV}$, where $M_{d}=1875.6 \mathrm{MeV}$ is the value of deuteron mass ${ }^{1}$. It can hardly be interpreted as a mere coincidence that the broadening of the peak caused by the "quasi-free" nucleon knockout in the experiment [252] can also be explained [250] by scattering $\mathrm{d}+\mathrm{d} \rightarrow \mathrm{d}+\mathrm{d} *$ with the participation of the same dibaryons as in the experiment [251]. Of course, these arguments can only be considered as indirect evidence in favor of the existence of light dibaryons. On the other hand, it must be emphasized that the criticism of Troyan's experiment presented in [253] also does not look convincing enough. Firstly, in [251] a larger number of events were studied than in [253]; secondly, in [253] , events with a poorer "cooling" of a highly excited dibaryon were used, i.e. the work is based on events with fewer secondary pions emitted by a highly excited dibaryonic system. And finally, even in spite of all that, significant variations in the measured cross sections were observed in [253], which can hardly be explained by simple statistical fluctuations. The above facts suggest that light dibaryons could have been present in a sufficiently large number already in some of the earlier experiments, but were not recognized due to insufficient spectral resolution of the corresponding experimental facilities. Drawing an analogy with the history of discoveries associated with the invention of the microscope, we can say that a medium that can contain a large number of interesting microscopic objects is presumably known, but a device that allows them to be clearly seen has not yet been created. Below we shall consider the demands that would have to be advanced to an experimental setup so that it could be used to obtain unambiguous answers to all the above questions.

In experiments on colliding beams of deuterons with momenta of the same modulus, the energy $q_{0}$ transferred in the reaction $\mathrm{d}+\mathrm{d} \rightarrow \mathrm{d}+\mathrm{d} *$ from one of the deuterons to the dibaryon being produced is

$$
q_{0}=\frac{M_{*}^{2}-M_{d}^{2}}{4 E}
$$

\footnotetext{
${ }^{1}$ Hereinafter, the system of units is used, in which $c=1$.
} 
where $E=\sqrt{P^{2}+M_{d}^{2}}$ is the deuteron energy in the laboratory coordinate system (l.c.s.). For the $n$-th excited level of the dibaryon $M_{*}=M_{d}+n \varepsilon$. Here $\varepsilon$ is the distance between the levels, which we will take equal to $10 \mathrm{MeV}$ [250]. The relation (35) allows one to estimate the influence of the instability of the beam energy $E$ on the accuracy of determining the value of $\varepsilon$. For this purpose, let us bring into correspondence the spread in energy $\Delta E$ of deuterons in beams and the inaccuracy of setting the level number $0<\delta<1$, which will describe the deviation of the measured level energy from its exact value, referred to $\varepsilon$. Substitution of $E \rightarrow E+\Delta E$ and $n \rightarrow n+\delta n$ into the relation (35) gives a quadratic equation for $n$, which determines the highest level for which the uncertainty in the distance between adjacent levels is does not yet exceed the value of $\delta$ :

$$
\frac{\Delta E}{E}=\frac{2 M_{d}+(2 n+\delta) \varepsilon}{2 M_{d} n+n^{2} \varepsilon} \delta .
$$

For $n \varepsilon \ll M_{d}$, this relation is reduced to a directly proportional relationship between the relative fluctuation of the beam energy and the relative error in measuring the distance between adjacent levels, caused by these fluctuations:

$$
\delta \approx \frac{\Delta E}{E} n .
$$

It turns out to be rather weak (so far, the influence of the beam momentum spread on the accuracy of measuring the $M_{*}$ dibaryon mass is not taken into account). For example, for $\Delta E=100 \mathrm{MeV}$, the error in determining the energy of the tenth level of a dibaryon is only $1 \mathrm{MeV}$ for the momentum of colliding beams at the level of $10 \mathrm{GeV}$ in l.c.s.

It is also useful to have the correspondence between the 4-momentum $q$ transferred to the nascent dibaryon, which can be measured experimentally, and the relativistic Mandelstam variable $t \equiv q_{0}^{2}-\mathbf{q}^{2}$. One can make sure after elementary but cumbersome calculations that for the experiments with colliding beams the following simple formula holds:

$$
t=2\left(E q_{0}-P q_{\|}\right) .
$$

Here $q_{\|}$is longitudinal, i.e. directed along the beams' axis, the component of the momentum transfer, which is directly responsible for the excitations. It can be seen from (37) that the processes of dibarion production from a kinematic point of view are possible at both negative and positive, as well as zero values of the varibale $t$, which seems to be extremely unlikely. It is clear that the dynamic theory of the processe should be based on the relativistic-invariant amplitude using the variable $t$, since the fact of dibarion production does not depend on the frame in which it is registered. In the absence of such a theory, the answer to the question of whether the dibaryons under consideration are produced, for example, ar zero values of the variable $t$, can only be given by experiment. Since the relativistic-invariant amplitude also depends on the Mandelstm variable $s$, the search for the region of localization of dibaryons should also be accompanied by a variation in the momentum of deuterons in colliding beams.

Traditionally, formula (35) is used to identify resonances, in which $M_{*}$ is the effective mass of decay products of a resonance. An individual resonance can be seen if an error of its mass determining, which includes its own width, and also takes into account the 


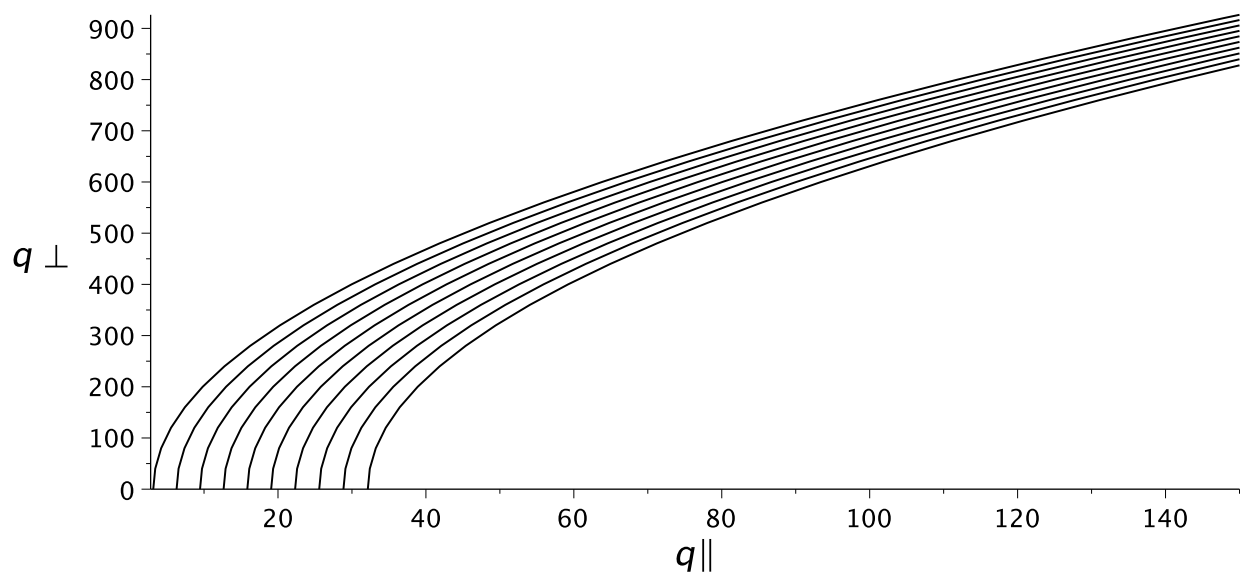

Fig. 25. Dependence of the transverse momenta of light dibaryons on their longitudinal momenta measured in $\mathrm{MeV}$ for an experiment with colliding beams with $P=3 \mathrm{GeV}$. The uppermost curve corresponds to the first excited level, and the lowest one corresponds to the tenth level.

measurement errors of the momenta of its decay products, is significantly less than distances to adjacent levels. It is clear that, to recognize resonances, one can also use the relationship (37), in which resonances are seen as separate lines. However, to formulate the requirements for the experimental setup, the most appropriate is a method of resonance recognition directly based on the quantities to be measured. Indeed, resonances, as well as the degree of reliability of their recognition, can also be seen on graphs of the dependence $q_{\perp}\left(q_{\|}\right)$with a direct indication of the measurement errors of longitudinal $q_{\|}$and perpendicular $q_{\perp}$ components of the momentum transfer. The exact analytical dependence $q_{\perp}\left(q_{\|}\right)$for colliding beams with equal momenta has the form

$$
q_{\perp}\left(q_{\|}\right)=\sqrt{\left(\frac{4 E^{2}+M_{*}^{2}-M_{d}^{2}}{4 E}\right)^{2}-M_{*}^{2}-\left(P-q_{\|}\right)^{2}} .
$$

This dependence is shown in Fig. 25 for the supposed theoretical values of the masses of dibaryons and $P=3 \mathrm{GeV}$. In a real experiment, values of the momenta of decay products of a dibaryon should be measured. For light dibaryons, these are the momenta $\mathbf{p}_{n}$ and $\mathbf{p}_{p}$ of the secondary neutron and proton. The transverse component of the momentum transferred to the dibaryon is equal to the transverse component of the total momentum of the secondary nucleons $q_{\perp}=p_{n \perp}+p_{p \perp}$, which is known with the measurement accuracy $\Delta p_{n, p \perp}=\Delta p_{n \perp}+\Delta p_{p \perp}$. In addition, the nonzero fluctuation of the transverse momentum of the beam $\Delta P_{\perp}$ makes a contribution to the measurement error of the transverse momentum transfer. Thus, the total measurement error of the transverse component of the momentum transfer is calculated by the formula

$$
\Delta q_{\perp}=\Delta p_{n \perp}+\Delta p_{p \perp}+\Delta P_{\perp}
$$


Similarly, taking into account that $q_{\|}=P-\left(p_{n \|}+p_{p \|}\right)$, we have

$$
\Delta q_{\|}=\Delta p_{n \|}+\Delta p_{p \|}+\Delta P_{\|}
$$

where $\Delta P_{\|}$is a value of the beam momentum fluctuations.

In paper [252], in which the light dibaryons could be observed at $t=-0.5 \mathrm{GeV}^{2}$, interactions of deuterons with momentum $P=8.9 \mathrm{GeV}$ with a stationary deuteron target were used. The colliding beam experiments at $|P|=3 \mathrm{GeV}$ correspond to the deuteron momentum $P=11.3 \mathrm{GeV}$ in experiments with the stationary target, which is close to the value in [252].

Using the formulas (35) and (37), it can be verified that the kinematics of colliding beams corresponds to similar events at $q_{\|}=100 \pm 20 \mathrm{MeV}$. In its turn, the formula (38) tells us that at $q_{\|}=100 \mathrm{MeV}$ the value of $q_{\perp}$ will be $755.8 \mathrm{MeV}$ for elastic d-d scattering, $743.2 \mathrm{MeV}$ for scattering with excitation of the first level, $730.3 \mathrm{MeV}$ for scattering with excitation of the second level, ... $631.0 \mathrm{MeV}$ - for the tenth level. In this range, the distances between the levels grow as follows: $12.3,12.6,12.9, \ldots 14.9,15.3 \mathrm{MeV}$. Thus, it can be seen that the measurement accuracy of the transverse momentum at a level of $3 \mathrm{MeV}$ is quite enough to register all the dibaryons described above.

To estimate the required accuracy of measuring the longitudinal component of the momenta of the secondary particles, the dependence (38) should be reversed, expressing $P-q_{\|}$in terms of $q_{\perp}$. One can check that the distances in the horizontal direction between the levels of dibaryons shown in Fig. 25, in this case only slightly more than $3 \mathrm{MeV}$ at full longitudinal momentum $P-q_{\|}$of the decay products at a level of $3000 \mathrm{MeV}$. This dictates very high requirements both for the measurement accuracy of the longitudinal components of momentum of the secondary particles and the degree of monochromaticity of the beam - at the level of $10^{-4}$. Nevertheless, even in this respect, the experiments with colliding beams have significant advantages in relation to experiments with the stationary target. In the latter case, a similar experiment would require increasing relative accuracy by a factor of 7 , because of the larger momentum $P$ of the accelerated beam and also because of a different analytical dependence $q_{\perp}\left(q_{\|}\right)$.

The requirements for the accuracy of measuring the longitudinal component of the momentum transfer very fast decrease with decreasing momentum of the colliding beams. So, if it is equal to $1 \mathrm{GeV}$, then the required relative accuracy becomes equal to $10^{-2}$. Since the exact range of values of the Mandelstam variable $s$, within which the production of light dibaryons is possible, we do not know, then it makes sense to set up an experiment even at lower than in the experiment [252] values of $s$.

Fig.25 suggests the possibility of searching for any dibaryons, including heavier ones, the existence of which quark models predict. For this, it is enough to use the fact of a distinct concentration events near some line $q_{\perp}\left(q_{\|}\right)$, described by formula (38), which should be very similar to those shown in Fig. 25 . 


\section{Proposal for the study of lightest neutral hypernuclei with strangeness -1 and $-2^{1}$}

Based on our recent study of the lightest neutral hypernuclei with strangeness -1 and -2 , we propose to look for the neutral hypernucleus ${ }_{\Lambda \Lambda}^{4} \mathrm{n}$ in deuteron-deuteron collisions which can be accessed by SPD NICA in the future. Some advantages and opportunities for hypernuclei and exotic hadrons in the double $K^{+}$production channels at NICA are addressed.

PACS: 21.80.+a, 21.30.Fe, 21.30.-x, 21.45.-v, 25.45.De

There have been significant progresses in the study of hypernuclei in both experiment and theory during the past decades. The discovery of a number of hypernuclei with $S=-1$ and $S=-2$ has greatly enriched our knowledge about the hyperon-nucleon and hyperon-hyperon interactions [254,255], and the search for stable hypernuclei still serves as a direct probe for many interesting physics with the presence of hyperons in nuclear matter.

So far, there is no evidence for a stable $(n, \Lambda)$ nor $(p, \Lambda)$ bound state for baryon number $A=2$, except for a resonance peak of about $2.06 \mathrm{GeV}$ seen in $d+\pi^{-}[256]$. The situation becomes more complicated in the 3-body or 4-body systems made of nucleons and hyperons. For $A=3$ in the sector of isospin $I=1,{ }_{\Lambda}^{3} \mathrm{He}=(p, p, \Lambda)$ and ${ }_{\Lambda}^{3} \mathrm{H}=(n, p, \Lambda)$ are found bound. The latter has a 3-body binding energy of $E_{3}=-2.45 \mathrm{MeV}$, which is just below the threshold of a deuteron plus an isolated $\Lambda$, i.e. $E_{2}=-2.20 \mathrm{MeV}$. In contrast, the existence of ${ }_{\Lambda}^{3} \mathrm{n}=(n, n, \Lambda)$ still needs confirmation [256].

For $A=4$ with $I=0$, the nonstrange tetraneutron, ${ }^{4} \mathrm{n}=(n, n, n, n)$, is naively suggested to exist by the stability of ${ }^{8} \mathrm{He}$ isotope, but so far has received only controversial experimental indications [257]. Calculations based on realistic neutron-neutron potentials [258-262] or potentials made artificially deeper to produce a dineutron ${ }^{2} \mathrm{n}$ do not support its stability due to the Pauli principle. To avoid the Pauli blocking effects and benefit from the convening role played by the hyperon, it is thus interesting to consider the stability of the doubly-strange tetra-baryon system, $T={ }_{\Lambda}{ }_{\Lambda}^{4} \mathrm{n}=(n, n, \Lambda, \Lambda)$ with $S=-2$ and $I=1$. In particular, the ground state should favor the spin-singlet assignments for both the $n n$ and the $\Lambda \Lambda$ pairs in order to satisfy the antisymmetrisation.

In the sector of $A=4$ and $S=-2$, namely the "double $\Lambda$ " hypernuclei, it includes the states, ${ }_{\Lambda \Lambda}^{4} \mathrm{He},{ }_{\Lambda \Lambda}^{4} \mathrm{H}$, and ${ }_{\Lambda \Lambda}^{4} \mathrm{n}$. Note that the "Nagara" event [263], has set a limit on the $\Lambda \Lambda$ effective attraction in ${ }_{\Lambda}{ }^{6} \mathrm{He}$ with a precise measurement of its binding energy. This may provide some constraints on lighter $S=-2$ systems [264-266], and in particular hypernuclei with $A=4$.

The ${ }_{\Lambda \Lambda}^{4} \mathrm{H}$ was studied in Ref. [267] by Filikhin and Gal, and it was found to be unbound within the models adopted. However, with a more sophisticated method for solving the four-body problem, the calculation by Nemura, Akaishi and Myint [268] found a small amount of binding below the threshold of dissociation into ${ }_{\Lambda}^{3} \mathrm{H}+\Lambda$. As stressed in Refs. [264,267,268], the interaction of $\Lambda \Lambda \leftrightarrow N \Xi \leftrightarrow \Lambda \Lambda$, which is sizeable in free $\Lambda \Lambda$ scatterings, will be suppressed in a dense nucleus due to the antisymmetrisation between the nucleons in the core and the nucleon in $N \Xi$. Such a Pauli suppression effect was

\footnotetext{
${ }^{1}$ This section is written by J.-M. Richard, Q. Wang and Q. Zhao; E-mail:zhaoq@ihep.ac.cn.
} 
invoked to explain the relatively weak binding of ${ }_{\Lambda \Lambda}^{6} \mathrm{He}$. However, since it requires a $(n \Lambda \Lambda)$ correlation it should not play a significant role in the limit of weak binding.

It is no doubt that the question of stability of the $A=4$ double- $\Lambda$ hypernuclei would be crucial for our understanding of the role played by hyperons in nuclear matter. While it is still controversial for model calculations of such a four-body problem in the regime of weak binding, we would propose that some general properties arising from the weakly binding systems involving the 2-body and 3-body bound-state energies may provide a guidance for a possible stability of ${ }_{\Lambda}^{4} \mathrm{n}$. Meanwhile, we propose a sensitive reaction process for the search for ${ }_{\Lambda \Lambda}^{4} \mathrm{n}$ in deuteron-deuteron scatterings, i.e. $d+d \rightarrow$ $K^{+}+K^{+}+{ }_{\Lambda \Lambda}^{4} \mathrm{n}$, which is accessible at NICA. After all, it would rely on the experimental study to decide the dedicate dynamics for such an exotic system.

As follows, we will first demonstrate that ${ }_{\Lambda \Lambda}^{4} \mathrm{n}$ may exist given the satisfaction of Thomas condition, where ${ }_{\Lambda \Lambda}^{4} \mathrm{n}$ is likely to be a "Borromean" system. We then propose and discuss its production mechanism in $d+d \rightarrow K^{+}+K^{+}+{ }_{\Lambda \Lambda}^{4} \mathrm{n}$. Advantages of using the double $K^{+}$production channels to probe exotic hypernuclei and exotic hadrons are also addressed. A brief summary is given in the end.

\subsection{Binding conditions for 3 and 4-body systems with strangeness -1 and -2}

The Thomas condition is referred to the relation between the range of nuclear forces and the ratio of 3-body to 2-body bound-state energies which was discovered by Thomas in 1935 [269]. Namely, it shows that the ratio of 3-body to 2-body bound-state energies, $E_{3} / E_{2}$, becomes very large if the range of the interaction decreases. In such a case one may have a deep 3-body binding of $E_{3} / E_{2} \rightarrow \infty$ for a given (short) range if the coupling $g$ approaches (from above) the minimal value $g_{2}$ required by the 2-body binding. Here, the coupling $g$ is defined by the potential energy $g \sum v\left(r_{i j}\right)$, where $v$ accounts for the attractive parts of the potential, and $r_{i j}$ denotes the inter-particle separation. Implication of the Thomas condition is that the minimal coupling $g_{3}$ to bind three particles is smaller than $g_{2}$. Therefore, it allows a coupling value of $g_{3}<g<g_{2}$, which will lead to a "Borromean" 3-body binding system. Namely, the 3-body system become bound but its two-body subsystems are unbound.

The rigorous boundaries on the allowed domain of coupling constants for the Borromean system have been studied in the literature [270], and the Borromean window as a function of the potential shape [271] or of the low-energy parameters of the pair interactions [272] have been investigated. In Ref. [273] it was shown that such a Borromean binding for 3-body (such as $n n \Lambda$ ) and 4-body (such as $n n \Lambda \Lambda$ systems may exist based on some general features implemented for the 2-body interactions.

While the detailed discussions on the Thomas conditions can be found in Refs. [273, $274]$, we only outline the key points which are relevant to the study of the $n n \Lambda \Lambda$ system. First, we adopt some simple potentials for the 2-body interaction:

$$
\begin{aligned}
& -g \exp (-\mu r) \quad \text { (exponential), } \\
& -g \exp (-\mu r) / r \quad \text { (Yukawa), } \\
& g \exp [-2 \mu(r-R)]-2 g \exp [-\mu(r-R)] \text { (Morse), }
\end{aligned}
$$

where for the Morse potential, we use $R=0.6$ for the illustration purpose. 
Table 4. Values (in $\mathrm{fm}$ ) adopted for the scattering length and effective range parameter in the two models.

\begin{tabular}{cccccc}
\hline \hline \multirow{2}{*}{ Pair } & \multicolumn{2}{c}{ ESC08 } & & \multicolumn{2}{c}{ CEFT } \\
\cline { 2 - 3 } \cline { 5 - 6 } & $\mathrm{a}$ & $r_{\text {eff }}$ & & $\mathrm{a}$ & $r_{\text {eff }}$ \\
\hline$n n$ & -16.51 & 2.85 & & -18.9 & 2.75 \\
$(n \Lambda)_{s=0}$ & -2.7 & 2.97 & & -2.9 & 2.65 \\
$(n \Lambda)_{s=1}$ & -1.65 & 3.63 & & -1.51 & 2.64 \\
$\Lambda \Lambda$ & -0.88 & 4.34 & & -1.54 & 0.31 \\
\hline \hline
\end{tabular}

We then solve the 2-body problem to reproduce the deuteron binding energy, and $n n$, $n \Lambda$ and $\Lambda \Lambda$ scattering lengths and effective ranges extracted by the Nijmegen-RIKEN model $[254,254,275]$ and the chiral effective field theory (CEFT) [276,277]. The values for the scattering lengths and effective ranges from these two models are listed in Table 11.1. It shows that these two models have similar results for the $n n$ and $n \Lambda$ interactions, but they produce quite different values for the $\Lambda \Lambda$ interaction. In particular, the effective range of $\Lambda \Lambda$ is remarkably small, and for a given scattering length, this eases the occurrence of Borromean binding involving a pair of $\Lambda$. However, in a more advanced CEFT study [278], the same group found a larger value for $r_{\text {eff }}$, and this modifies the conclusions for the 3-body and 4-body systems at the edge of binding. Hopefully, some new experimental results, e.g., from final-state correlations in heavy-ion collisions [279] would allow for a better tuning of the models. The main results can be outlined as follows:

- The model reproduces the observed binding of the ${ }^{2} \mathrm{H}$ and ${ }_{\Lambda}^{3} \mathrm{H}$ systems. For ${ }_{\Lambda}^{3} \mathrm{H}$ both spin $s=1 / 2$ and $s=3 / 2$ are found bound since there is no much difference between the spin-triplet and the spin-averaged nucleon-hyperon interactions.

- We find that ${ }_{\Lambda}^{3} \mathrm{H}$ with isospin $I=1$ and spin $s=1 / 2$ is marginally unbound. However, it may become bound if some masses increase by about $10 \%$. Namely, the unequal masses between nucleon and hyperon will bring more binding.

- We also find that ${ }_{\Lambda}^{3} \mathrm{n}$ is marginally unbound. Our results for ${ }_{\Lambda}^{3} \mathrm{n}$ agree with the conclusions of the recent studies [280-282].

- The state ${ }_{\Lambda \Lambda}^{4} \mathrm{H}$ with isospin $I=0$ is found weakly bound (about $3 \mathrm{MeV}$ ) in the Nijmegen-RIKEN model, and slightly more (about $9 \mathrm{MeV}$ ) in the CEFT one. The state ${ }_{\Lambda \Lambda}^{4} \mathrm{H}$ with isospin $I=1$ and ${ }_{\Lambda \Lambda}^{4} \mathrm{n}$ deviate from binding by a very small amount with the Nijmegen-RIKEN parameters, but become bound by about $1 \mathrm{MeV}$ with the CEFT parameters.

The above analysis is based on some general properties arising from few-body systems. In particular, the satisfaction of the Thomas condition is crucial for the stability of ${ }_{\Lambda \Lambda}^{4} \mathrm{n}$ as a Borromean system. However, it should be noted that the detailed dynamics for the nucleon-hyperon and hyperon-hyperon interactions will decide whether ${ }_{\Lambda \Lambda}^{4} \mathrm{n}$ could exist at all. At this moment, there are still significant discrepancies between some of the most popular models. For instance, as shown in Table 11.1, the $\Lambda \Lambda$ scattering length and 
effective range determined by the Nijmegen-RIKEN model and CEFT model turn out to be signficantly different. It indicates that experimental constraints on the $\Lambda \Lambda$ interaction is desired.

Another point needs to be addressed is that so far the 3-body forces have not been considered. Whether or not they contain an attractive component would be crucial for the stability of close-to-binding systems. In case that some spin-dependence of the 3-body forces can play a significant role, it would keep the spin $s=1 / 2$ state of ${ }_{\Lambda}^{3} \mathrm{H}$ bound and move the $s=3 / 2$ in the continuum. It is also possible that the 3 -body and $n$-body forces with $n>3$ contain a short-range repulsive component. This is due to the Pauli exclusion of the constituent quarks when several hyperons (or several hyperons and nucleons) overlap within a small distance. The repulsive component seems to be necessary in large systems containing strangeness [283]. While the calculations of hyperon-nucleon and hyperon-hyperon forces should be pushed to higher order within theoretical models, experimental search for these strangeness -2 hypernuclei would provide crucial constraints on the model parameters.

11.2. Production mechanism for ${ }_{\Lambda \Lambda}^{4} \mathrm{n}$ and advantages of double $K^{+}$productions

We turn to the possible experimental search for ${ }_{\Lambda \Lambda}^{4} \mathrm{n}$ and propose a production mechanism which can be accessed at SPD NICA. In Ref. [273] we have shown that the deuterondeuteron collisions around the energy region above $E_{c m} \simeq 5.2 \mathrm{GeV}$ is favored to produce ${ }_{\Lambda}^{4} \mathrm{n}$ with the total cross section of about $2.5 \mathrm{nb}$. Here, based on the same analysis we try to clarify some key points and make a rough estimate of its production rate at the kinematics of SPD NICA.

As mentioned earlier, the quantum numbers of the ground state ${ }_{\Lambda \Lambda}^{4} \mathrm{n}$ will favor $J^{P}=$ $0^{+}$, where the neutron pair and $\Lambda$ pair have spin 0 , namely, their spins are anti-parallel, respectively. Meanwhile, the total isospin is $I=1$. Thus, the total wavefunction of the ground state is anti-symmetric under the interchange of the two nucleons or the two $\Lambda$. In principle, one has to construct a dynamic wavefunction for the $(n, n, \Lambda, \Lambda)$ system, which is a nontrivial work and strongly model-dependent due to the unknown $\Lambda \Lambda$ interactions. But for the purpose of making an estimate of the production rate, we can simply introduce a momentum distribution for the $n \Lambda$ clusters for the $(n, n, \Lambda, \Lambda)$ system [273].

The most ideal reaction for producing ${ }_{\Lambda}{ }^{4} \mathrm{n}$ should be $d+d \rightarrow K^{+}+K^{+}+T$ which is an extremely clean process since the background processes involving the $K^{+} K^{-}$productions become irrelevant. It makes the measurement of the missing mass spectrum recoiling against the $K^{+} K^{+}$pairs sensitive to the existence of any pole structure in the $n n \Lambda \Lambda$ system. The transition matrix element can be expressed as

$$
\begin{aligned}
\mathcal{M} & =\int \psi_{T}^{*}\left(\boldsymbol{p}_{1}^{\prime}, \boldsymbol{p}_{2}^{\prime}, \boldsymbol{p}_{3}^{\prime}, \boldsymbol{p}_{4}^{\prime} ; \boldsymbol{P}_{T}^{\prime}\right) \psi_{K_{1}}^{*}\left(\boldsymbol{P}_{K_{1}}\right) \psi_{K_{2}}^{*}\left(\boldsymbol{P}_{K_{2}}\right) \hat{\mathcal{O}}\left(\boldsymbol{p}_{1}^{\prime}, \boldsymbol{p}_{3}^{\prime}, \boldsymbol{p}_{1}, \boldsymbol{p}_{3}, \boldsymbol{P}_{K_{1}}, \boldsymbol{P}_{K_{2}}\right) \\
& \times \psi_{d_{1}}\left(\boldsymbol{p}_{1}, \boldsymbol{p}_{2} ; \boldsymbol{P}_{d_{1}}\right) \psi_{d_{2}}\left(\boldsymbol{p}_{3}, \boldsymbol{p}_{4} ; \boldsymbol{P}_{d_{2}}\right) \delta\left(\boldsymbol{P}_{T}^{\prime}+\boldsymbol{P}_{K_{1}}+\boldsymbol{P}_{K_{2}}-\boldsymbol{P}_{d_{1}}-\boldsymbol{P}_{d_{2}}\right) \\
& \times \delta\left(\boldsymbol{p}_{1}+\boldsymbol{p}_{2}-\boldsymbol{P}_{d_{1}}\right) \delta\left(\boldsymbol{p}_{3}+\boldsymbol{p}_{4}-\boldsymbol{P}_{d_{2}}\right) \delta\left(\boldsymbol{p}_{1}^{\prime}+\boldsymbol{p}_{2}^{\prime}+\boldsymbol{p}_{3}^{\prime}+\boldsymbol{p}_{4}^{\prime}-\boldsymbol{P}_{T}^{\prime}\right) \\
& \times \delta\left(\boldsymbol{p}_{2}-\boldsymbol{p}_{2}^{\prime}\right) \delta\left(\boldsymbol{p}_{4}-\boldsymbol{p}_{4}^{\prime}\right) d \boldsymbol{p}_{1} d \boldsymbol{p}_{2} d \boldsymbol{p}_{3} d \boldsymbol{p}_{4} d \boldsymbol{p}_{1}^{\prime} d \boldsymbol{p}_{2}^{\prime} d \boldsymbol{p}_{3}^{\prime} d \boldsymbol{p}_{4}^{\prime}
\end{aligned}
$$


where the kinematic variables are defined in Fig. 26. Note that Fig. 26 illustrates one of the leading transitions favored by the ${ }_{\Lambda}^{4} \mathrm{n}$ production in the central deuteron-deuteron collisions.

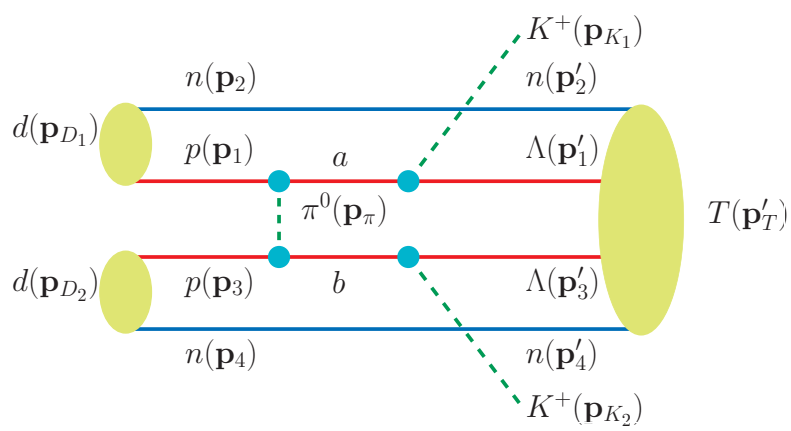

Fig. 26. (Color online) Mechanism for $T$ production in $d d$ collisions.

In Fig. 27 the total cross section is estimated by the leading transition process of Fig. 26. As the leading-order test the relative internal momenta between the proton and neutron inside the incident deuterons has been neglected. Namely, the Fermi motion inside the initial deuterons is neglected. Also, the final-state interactions among the finalstate baryons are overlooked, which means that the neutrons are treated as spectators and their contributions to the amplitude will be via the convolution of the final-baryon momentum distributions. The $S_{11}(1535)$ resonance is included in the transition amplitude which is found to be relatively small, mainly due to the smaller couplings to $\pi N$ and $K \Lambda$. Since other processes with intermediate $N^{*}$ excitations may contribute, our estimate including only the Born term and $S_{11}(1535)$ excitations can be regarded as a conservative estimate of the production cross section for ${ }_{\Lambda}^{4} \mathrm{n}$.

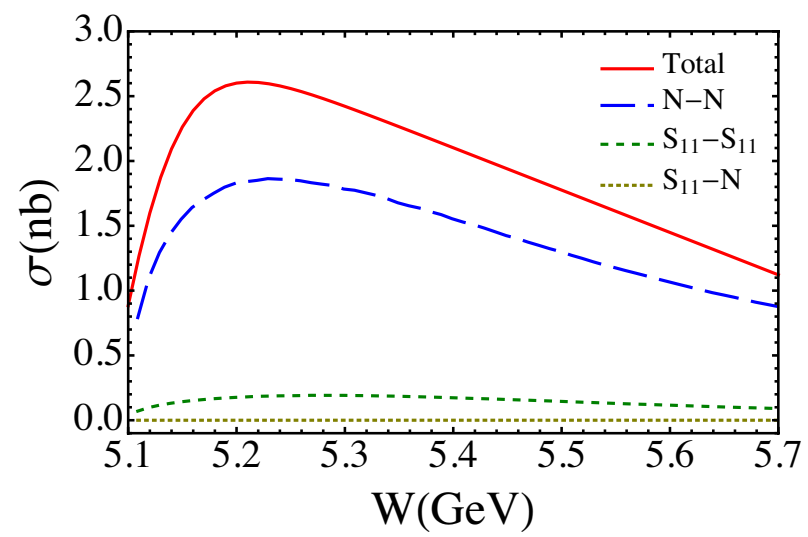

Fig. 27. (Color online) Total cross section for $\left.d+d \rightarrow K^{+}+K^{+}+(n, n, \Lambda, \Lambda)\right)$. From upper to lower the curves stand for the total cross sections of the full calculations, exclusive process from the nucleon Born terms, exclusive process from the double $S_{11}$ (1535) excitations, and exclusive process from the one Born transition and one $S_{11}(1535)$ excitation. 
In Fig. 28 the $K^{+} K^{+}$missing mass spectra for the recoiled $(n, n, \Lambda, \Lambda)$ at different energies are shown above the production threshold. The peak position is located at the four baryon $n n \Lambda \Lambda$ threshold since only a momentum distribution for $\Lambda \Lambda^{4} \mathrm{n}$ is considered. However, our estimate is sufficient to demonstrate the behavior of the correlated system recoiled by the $K^{+}$pair. For uncorrelated $K^{+} K^{+}$events, i.e. the final-state $(n, n, \Lambda, \Lambda)$ are not bound, there would no peak in the missing mass spectrum.

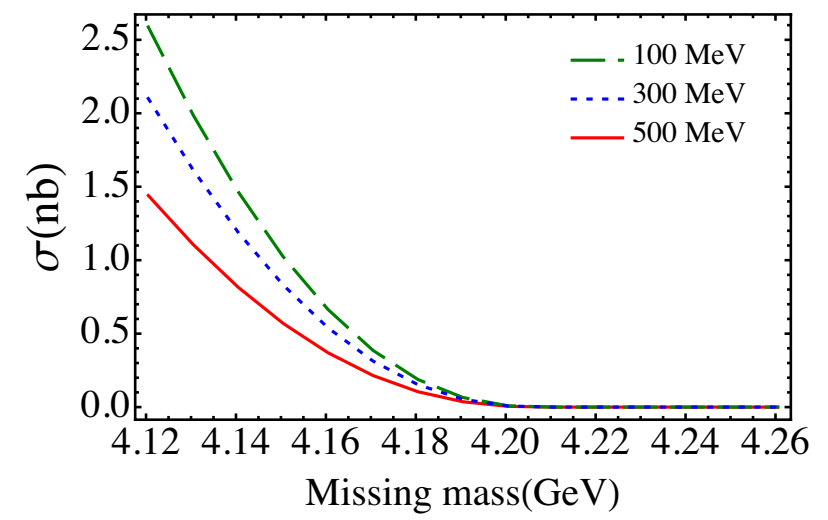

Fig. 28. (Color online) The missing mass spectra for the recoiled $(n, n, \Lambda, \Lambda)$ at different energies above the production threshold in $d+d \rightarrow K^{+}+K^{+}+(n, n, \Lambda, \Lambda)$

The c.m. energy at SPD NICA starts at $E_{c m}=6.7 \mathrm{GeV}$ with a luminosity of $L=10^{27} \mathrm{~cm}^{-2} \mathrm{~s}^{-1}$. We estimate that the total cross section at $E_{c m}=6.7 \mathrm{GeV}$ will drop about one order of magnitude compared with the peak value of about $2 \mathrm{nb}$. Thus, the events expected in one-year runtime are

$$
N=\sigma_{\text {total }} \times L \times t=0.2 \mathrm{nb} \times 10^{27} \mathrm{~cm}^{-2} \mathrm{~s}^{-1} \times 1 \text { year } \simeq 6.3,
$$

which is a small event counting. It could be even smaller taking into account the detection efficiency which generally will reduce the event counting by one order of magnitude. However, if the luminosity can reach $10^{29}$, which is an approximate average between the lower limit of $10^{27} \mathrm{~cm}^{-2} \mathrm{~s}^{-1}$ and the upper limit of $2 \times 10^{30} \mathrm{~cm}^{-2} \mathrm{~s}^{-1}$, the event counting can be significantly increased:

$$
N_{m}=\sigma_{\text {total }} \times L \times t=0.2 \mathrm{nb} \times 10^{29} \mathrm{~cm}^{-2} \mathrm{~s}^{-1} \times 1 \text { year } \simeq 630 ，
$$

which is sufficient for establishing the state. For the highest luminosity, one would expect about 12000 events in one-year runtime. Even though the detection efficiency will reduce the events, there will be tens to hundreds of events to count.

It shows that the double $K^{+}$production channel has great advantages for the study of hypernuclei and exotic hadrons. Apart from the proposed process, $d+d \rightarrow K^{+}+$ $K^{+}+(n, n, \Lambda, \Lambda)$, it is also interesting to look at the proton-proton collisions, $p+p \rightarrow$ $K^{+}+K^{+}+\Lambda+\Lambda$, where the missing mass spectrum of $K^{+} K^{+}$also provides a clean and direct way to search for the di-baryon $\Lambda \Lambda$, or to study the $\Lambda \Lambda$ interactions.

For the proton-deuteron collisions, the double $K^{+}$channel is $p+d \rightarrow K^{+}+K^{+}+n+$ $\Lambda+\Lambda$. The recoiled part of the double $K^{+}$is $n \Lambda \Lambda$. It is also unknown whether such an 
exotic system, i.e. $H$ di-baryon, can exist or not. A direct measurement of such a system would provide rich information about both $\Lambda \Lambda$ and $n \Lambda$ interactions. Nevertheless, notice that the final states have access to the $n K^{+}$invariant mass spectrum. The exclusive measurement of this process can also tell whether the light pentaquark state $\Theta^{+}(1540)$ exists or not.

\subsection{Summary}

The stability of ${ }_{\Lambda}^{3} \mathrm{n}$ and, more likely, of $T={ }_{\Lambda}^{4} \mathrm{n}$ is within the uncertainties of our knowledge of the baryon-baryon interaction. Although many effects need to be considered in order to refine the predictions, we propose that direct experimental evidence would be extremely useful for model constraints and a better understanding of the hyperon dynamics within nuclear matter. We also emphasize the advantages of the double $K^{+}$ production channels as a probe for topical exotic hypernuclei and exotic hadron studies. The SPD NICA facility would be the ideal place for such an experimental effort in the future. 


\section{Problems of soft $p p$ interactions ${ }^{1}$}

Experiments are proposed directed on solution of three main problems of physics of soft $p p$ interactions: understanding/description of baryon spectra in $p p$ collisions, evolution of $\left\langle P_{T}^{2}>\right.$ $-x_{F}$ correlations with energy growth and two-particle $P_{T}$ correlations.

PACS: 24.10.Lx, 13.85.Ni, 14.20.-c

Description of proton spectra in hadron-nucleon interactions is a long standing problem of high energy physics. By tradition, three reggeon phenomenology is used for its solution at $x_{F} \rightarrow 1$ (see Ref. [284]). Especially, it is assumed that the three pomeron graph $(\mathrm{PPP})$ is responsible for the high mass diffraction dissociation. The non-vacuum reggeon - two pomeron's graph (RPP) is connected with so-called low mass diffraction dissociation. The three non-vacuum reggeon's graph (RRR) gives a contribution at $x_{F} \geq$ 0.8. A.B. Kaidalov and O.I. Piskunova [285] proposed a method of the spectra description in the central region in the framework of the Quark-Gluon-String model. Up to now various Monte Carlo models cannot describe the spectra sufficiently well [286]. All of them are used the LUND fragmentation scheme [287] for a treatment of decays of quark-diquark strings. An example of the description is presented in Fig. 1 where Pythia 6.4 [288] and Geant4 FTF model's predictions are shown.

The Geant4 FTF model [289] considers RRR graphs. As seen, the Pythia model gives abnormal humps at $y \sim 1.7$ and 2.3 at $P_{l a b}=158$ and $400 \mathrm{GeV} / \mathrm{c}$, correspondently. Thus, detailed experimental data at low energies, where the diffractions are dominant processes, are highly desired for a development of the theoretical models.

The most impressive experimental data on $p p$ interactions were presented in the last decade by the NA61/SHINE collaboration for $\sqrt{s_{N N}}=6.2,7.6,8.8,12.3$ and 17.3 $\mathrm{GeV}$ [290]. It is pitty, but the data are not sufficiently detailed. Before there were data at $P_{l a b}=12$ and $24 \mathrm{GeV} / \mathrm{c}$ [291]. They were based on low statistics according to a modern point of view.

On the whole, we can say that the FTF model of Geant4 toolkit describes quite well the multiplicities and kinematical spectra of produced particles in proton-proton interactions in a wide laboratory energy range from $1 \mathrm{GeV}$ up to $1000 \mathrm{GeV}$.

Another problem, recognized by us, was observed in $\left\langle P_{T}^{2}\right\rangle-x_{F}$ correlations in $p p$ interactions. The NA61/SHINE collaboration did not provide corresponding experimental data for the correlations, though, they can be extracted in principle. The NA49 collaboration presented the needed data [293-295] only at the page -

http://spshadrons.web.cern.ch/ppdata.html. The data are very different from analogous data by the LEBC-EHS collaboration [292] at $\sqrt{s_{N N}}=27.5 \mathrm{GeV}$. The models cannot describe the last data quite well.

As seen in Fig. 2, the models describe the general behaviour of the data except the correlations for protons. Predictions of the models are close to each other and to the data for $\pi^{+}, \pi^{-}, K^{+}$and $K^{-}$mesons at $\sqrt{s_{N N}}=17.3 \mathrm{GeV}$. The FTF model overestimates the correlations for protons at $x_{F} \geq 0.2$, and does not reproduce the hump at $x_{F} \sim 0$. The Pythia model gives the hump, but it is essential lower than the experimental data. In other regions of $x_{F}$, the Pythia model does not describe the shape of the data. At

\footnotetext{
${ }^{1}$ This section is written by A. Galoyan and V. Uzhinsky.
} 

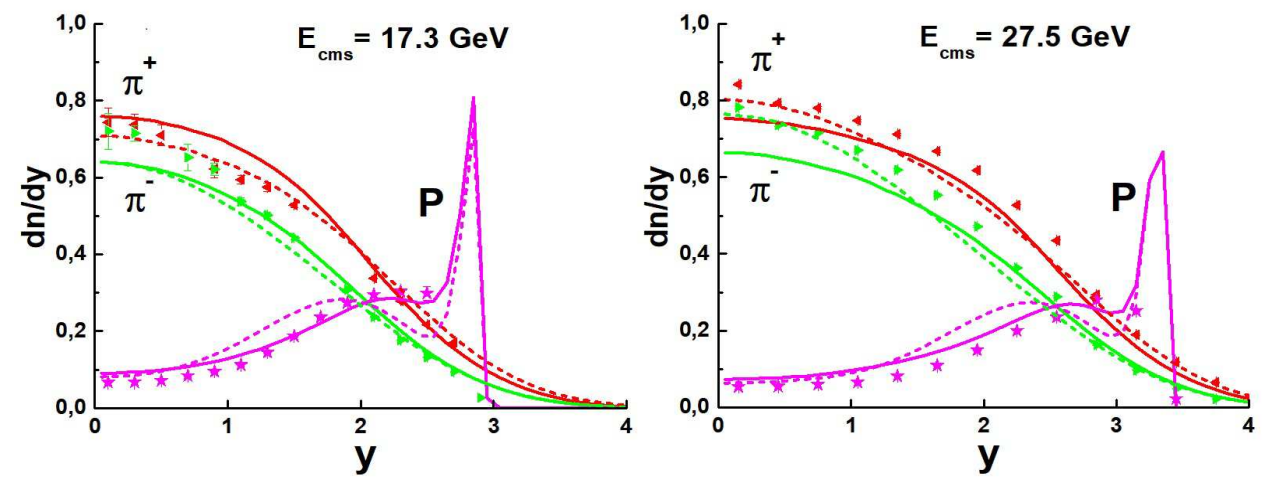

Fig. 29. Distributions of $\pi^{ \pm}$and protons on rapidity in the center-of-mass system of $p p$ interactions at $P_{l a b}=158$ and $400 \mathrm{GeV} / \mathrm{c}$. Points are experimental data [290,292] without systematical errors. Solid and dashed curves are FTF and Pythia 6.4 model calculations.
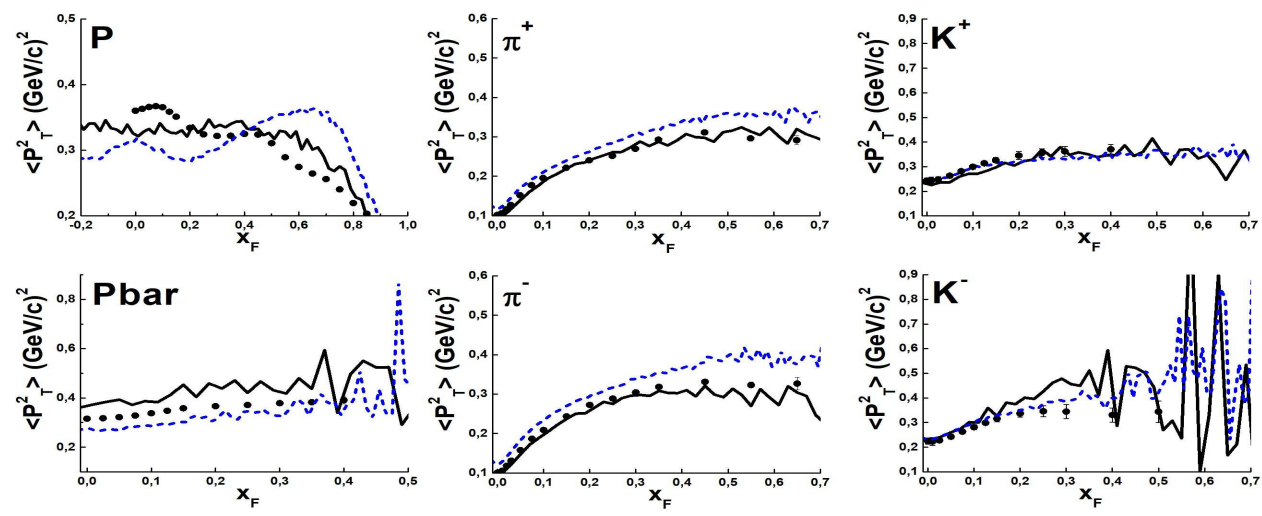

Fig. 30. $\left\langle P_{T}^{2}\right\rangle-x_{F}$ correlations in $p p$ interactions at $\sqrt{s_{N N}}=17.3 \mathrm{GeV}$. Points are the NA49 data [293-295]. Solid and dashed lines are FTF and Pythia 6.4 model calculations.

higher energy, the situation becomes worse for the proton correlation. This shows that both models have a problem with baryon production in $p p$ interactions.

It is interesting to look at evolution of the correlations with energy. In Fig. 3 we present $\left\langle P_{T}^{2}>-x_{F}\right.$ correlations at various energies. As seen, there is a smooth evolution, though there is a change of process contributions with energy in the FTF model. Quark exchange processes and one-vertex diffractions dominate at low energies. At higher energies, the diffraction is stay on the same lavel, but an yield of non-diffractive processes is increased.

In order to clarify a nature of the correlations, we propose to study two-particle $P_{T}$ correlations in soft interactions. The correlation can be also studied by current experiments at RHIC and the LHC.

As known, two-particle correlations were intensively used in the past at study of particles and jets in high energy physics (see, for example, [296-300]). At the present time, there are well developed methods of jet recognition $[301,302]$ and analysis of thier properties. More complicated situation takes place at sufficiently lower energies, especially for 

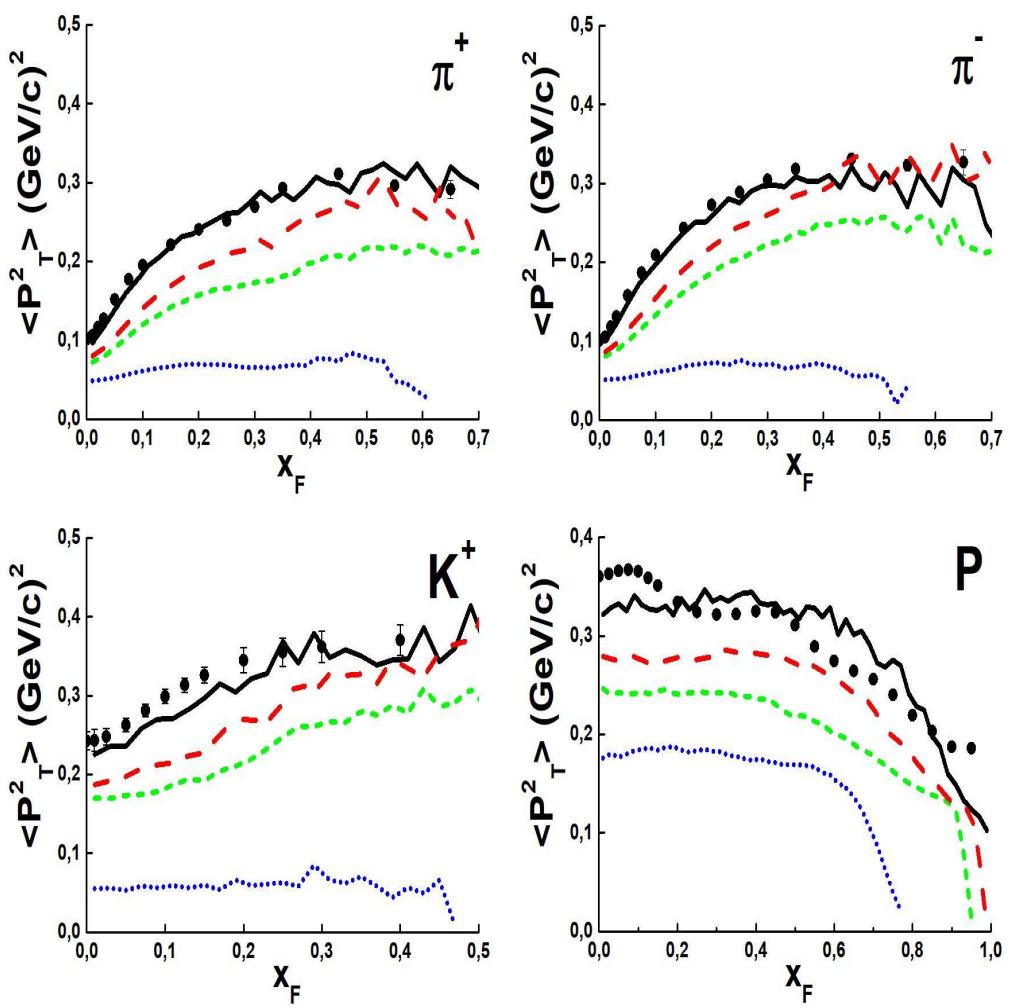

Fig. 31. $\left\langle P_{T}^{2}\right\rangle-x_{F}$ correlations in $p p$ interactions at $\sqrt{s_{N N}}=3.2,6.3,8.8$ and $17.3 \mathrm{GeV}$ (lines from bottom to top). Points are the NA49 data [293-295]. Lines are FTF model calculations.

nucleus-nucleus interactions, when hard jets cannot be produced. Though, some methods of high energies can be adjusted for lower energies. Instead of jets with high $P_{T}$, one can choose as a trigger a particle of any type in an event. An associated particle can be choosen in the same manner in the event, where the trigger paticle is produced.

In general, two-particle $P_{T}$ correlation function can be determined as:

$$
C\left(\vec{P}_{T}^{t r}, \vec{P}_{T}^{a s}\right)=\frac{1}{N_{t r}} \frac{d N(t r, a s)}{d^{2} P_{T}^{t r} d^{2} P_{T}^{a s}},
$$

where $\vec{P}_{T}^{t r}$ is a transverse momentum of a trigger particle. $\vec{P}_{T}^{a s}$ is a transverse momentum of an associated particle. $N_{t r}$ is a number of the trigger particles. $N(t r, a s)$ is a number of pairs - trigger particles and associated particle having pre-determined values of $\vec{P}_{T}^{t r}$ and $\vec{P}_{T}^{a s}$.

The function $C$ is a function of 4 independent variables. Though, accounting azimuthal symmetry of interactions of unpolarized particles there must be only 3 independent variables. We propose to use as the variables the module of transverse momentum of the trigger particle $\left(\left|\vec{P}_{T}^{t r}\right|\right)$, and 2 projections of the vector $\vec{P}_{T}^{a s}$ on the direction of the vector $\vec{P}_{T}^{t r}$, and on the direction perpendicular to $\vec{P}_{T}^{t r}$ (see Fig. 4 ). In Fig. 4 we choose $\Lambda$ as a trigger particle, and $K$-meson or $\pi$-meson as associated particles. 


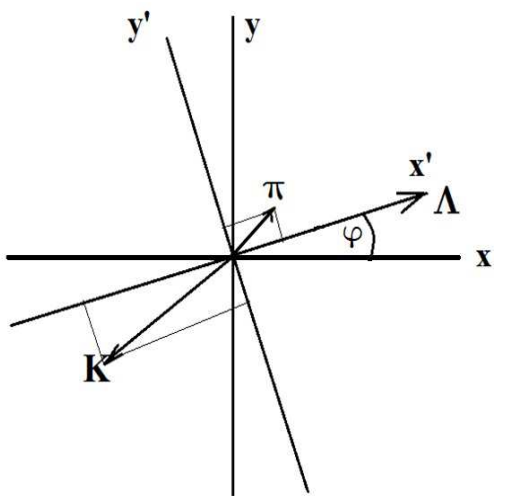

Fig. 4

$$
\begin{aligned}
P_{T, L}^{a s} & =\vec{P}_{T}^{a s} \cdot \vec{P}_{T}^{t r} /\left|\vec{P}_{T}^{t r}\right|, \\
P_{T, T}^{a s} & =\left|\vec{P}_{T}^{a s} \otimes \vec{P}_{T}^{t r}\right| /\left|\vec{P}_{T}^{t r}\right|
\end{aligned}
$$

Instead of calculations of scalar and vector products, one can use the following method: Determine the azimuthal angle of flying of the trigger particle $-\phi=\arctan P_{T, y}^{t r} / P_{T, x}^{t r}$. Make the Euler transform of the coordinate system, and find new components of $\vec{P}_{T}^{a s}$.

$$
\begin{gathered}
P_{T, x^{\prime}}^{a s}=P_{T, x}^{a s} \cdot \cos \phi+P_{T, y}^{a s} \cdot \sin \phi, \\
P_{T, y^{\prime}}^{a s}=-P_{T, x}^{a s} \cdot \sin \phi+P_{T, y}^{a s} \cdot \cos \phi .
\end{gathered}
$$

It is obvious, that the new components of $\vec{P}_{T}^{t r}$ will be $P_{T, x^{\prime}}^{t r}=\left|\vec{P}_{T}^{t r}\right|$ and $P_{T, y^{\prime}}^{t r}=0$. In the following, we will omit subscripts " $T$ ", and apostrophes of $x$ and $y$ for the new components of vectors.

Trigger and associated particles can be chosen in various rapidity/pseudo-rapidity windows. Types of the particles can or cannot coincide. It is useful to consider also the correlation functions integrated on one or two variables.

Let us consider a connection of the functions with transverese momentum generation mechanism (see Fig. 5). Let us take a baryon $(\Lambda)$ as a trigger particle. The transverse momentum of the baryon $\left(\vec{P}^{\Lambda}\right)$ is a sum of the transverese momentum of diquark $\left(\vec{P}_{q q}\right)$ after previouse fragmentation steps and the transverse momentum of $s$-quark $\left(P_{s}\right)$ produced from the vacuum: $\vec{P}^{\Lambda}=\vec{P}_{q q}+\vec{P}_{s}$. The transverse momentum of an associated hadron $\vec{P}^{a s}=-\vec{P}_{s}+\vec{P}_{q} . \vec{P}_{q}$ is a transverse momentum of a quark produced at the next fragmentation step from the vacuum.

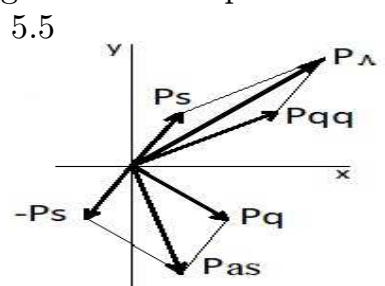

Fig. 5

According to the Schwinger's model [303] implemented in the LUND fragmentation model [287] distributions of $\vec{P}_{s}$ and $\vec{P}_{q}$ are Gaussian ones. Assume for simplicity, that distribution of $\vec{P}_{q q}$ has also Gaussian shape.

In the case, a probability density of the process is given by the expression:

$$
\left.\left.\propto \exp \left(-\overrightarrow{\left(P^{t r}\right.}\right)^{2} / \sigma_{1}-\overrightarrow{\left(P^{a s}\right.}\right)^{2} / \sigma_{2}+\frac{\sigma_{1} \sigma_{2}}{2 \sigma_{1}+\sigma_{2}}\left(\vec{P}^{t r} / \sigma_{1}-\vec{P}^{a s} / \sigma_{2}\right)^{2}\right) .
$$

Choosing $x$-axis direction along the vector $\vec{P}^{t r}$, we have:

$$
W\left(\vec{P}^{t r}, \vec{P}^{a s}\right) \propto \exp \left(-\frac{\sigma_{1}+\sigma_{2}}{\sigma_{2}\left(2 \sigma_{1}+\sigma_{2}\right)}\left[P_{x}^{a s}+\frac{\sigma_{2}}{\sigma_{1}+\sigma_{2}} P^{t r}\right]^{2}-\frac{\sigma_{1}+\sigma_{2}}{\sigma_{2}\left(2 \sigma_{1}+\sigma_{2}\right)}\left(P_{y}^{a s}\right)^{2}\right)
$$

Using Exp. 50, one can obtain for average values of projections of vector $\vec{P}^{a s}$ on $x$ 
and $y$ axes the following expressions:

$$
\begin{gathered}
<P_{x}^{a s}>=-\frac{\sigma_{2}}{\sigma_{1}+\sigma_{2}} P^{t r}, \\
<\left(P_{y}^{a s}\right)^{2}>=\frac{1}{2} \frac{2 \sigma_{1}+\sigma_{2}}{\sigma_{1}+\sigma_{2}} \cdot \sigma_{2} .
\end{gathered}
$$

It is useful to consider the following cases:

1) $\sigma_{1}=0, \quad<\left(P_{y}^{a s}\right)^{2}>=\sigma_{2} / 2, \quad\left\langle P_{a s, x}>=-P_{t r}\right.$

2) $\sigma_{1}=\sigma_{2},\left\langle\left(P_{y}^{a s}\right)^{2}>=\frac{3}{4} \sigma_{2}, \quad\left\langle P_{x}^{a s}>=-\frac{1}{2} P^{t r}\right.\right.$

3) $\sigma_{2} \simeq 0, \quad<\left(P_{y}^{a s}\right)^{2}>\simeq 0, \quad<P_{a s, x}>\simeq 0$

In the first case, we assume that diquarks have no transverse momentum. The second case corresponds to the assumptions that average momenta of quarks and diquarks are equal. The last one shows the obvious results, when transverse momenta of sea quarks are equal zeros.

Let us test the method on events generated by Pythia 6.4 and Geant4 FTF models at $\sqrt{s_{N N}}=10 \mathrm{GeV}$. We chose for the testing $\Lambda$-hyperons as trigger particles, and considered anti- $\Lambda$ hyperons, $K$-mesons and $\pi$-mesons as associated particles. We expected that there will be an essential difference between the model preductions due to the difference in the baryon production mechanisms. As seen in Fig. 6, it is so. We present in Fig. $6<P_{x}^{a s}>$ as functions of triggered $\Lambda$ momentum for various associated particles.

As seen, first of all, the correlations are approximately linear functions of $P^{t r}$, as it was expected. A large deviation between the model results takes place for $\Lambda$ correlations with $\bar{\Lambda}, K^{0}, K^{+}$and $\pi^{-}$mesons. The models give close predictions for $\Lambda$ correlations with $K^{-}$and $\pi^{+}$mesons. In general, the FTF model predicts strongest correlations between baryons and anti-baryons. Other strong correlations are between triggered $\pi$ mesons and baryons. The correlations are weaker in the Pythia model. There is practically no correlations between $\Lambda$ and $\bar{\Lambda}$ hyperons in the Pythia model.

According to the FTF model, there is an evolution of the correlations from strong to less strong ones in the energy range $\sqrt{s_{N N}}=3-15 \mathrm{GeV}$. It is connected with dying out of the quark exchange processes in the FTF model. At higher energies the correlations become "frozen". A next step in the function's evolution can be at copious gluon production.

Correlations of $<\left(P_{y}^{a s}\right)^{2}$ with $P^{t r}$ give a possibility of a direct check of the Schwinger's mechanism. The correlations are presented in Fig. 7.

As seen, the correlations are practically constant. The model predictions are close to each other for $\pi^{ \pm}$and $K^{ \pm}$mesons. There is an essential difference between the predictions for $\bar{\Lambda}$ hyperons and $K^{0}$ mesons.

The correlations evolve in the energy range $\sqrt{s_{N N}}=3-15 \mathrm{GeV}$ in the FTF model. We believe all of the considered correlations can be studied at the SPD experiment. 

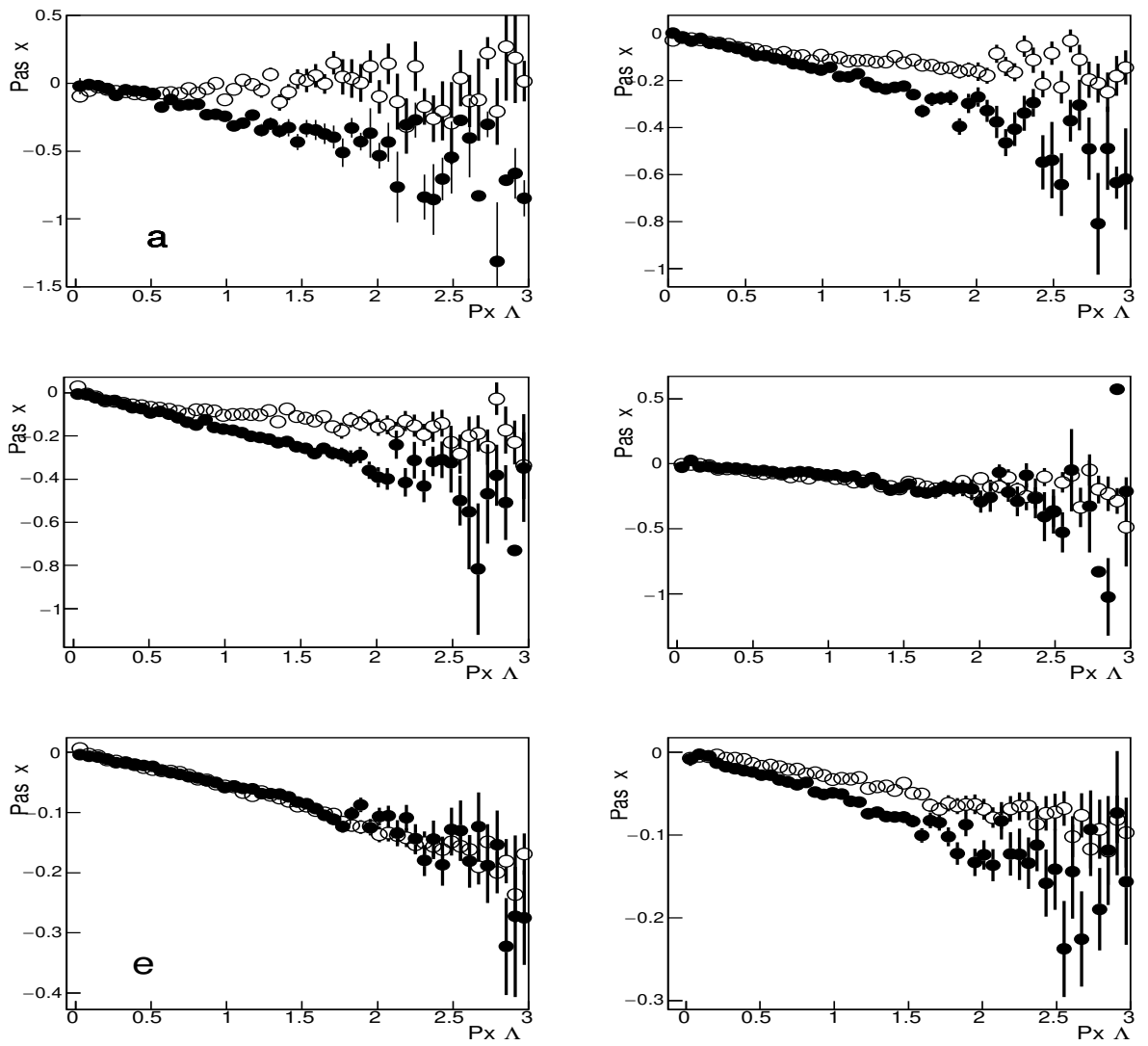

Fig. 34. $\left\langle P_{x}^{a s}>\right.$ as functions of triggered $\Lambda$ momentum for associated particles $-\bar{\Lambda}, K^{0}, K^{+}$, $K^{-}, \pi^{+}$and $\pi^{-}$, figures $a, b, c, d, e$ and $f$, respectively, in $p p$ interactions at $\sqrt{s_{N N}}=25 \mathrm{GeV}$. Open and filled symbols are Pythia and FTF calculation results, respectively. 

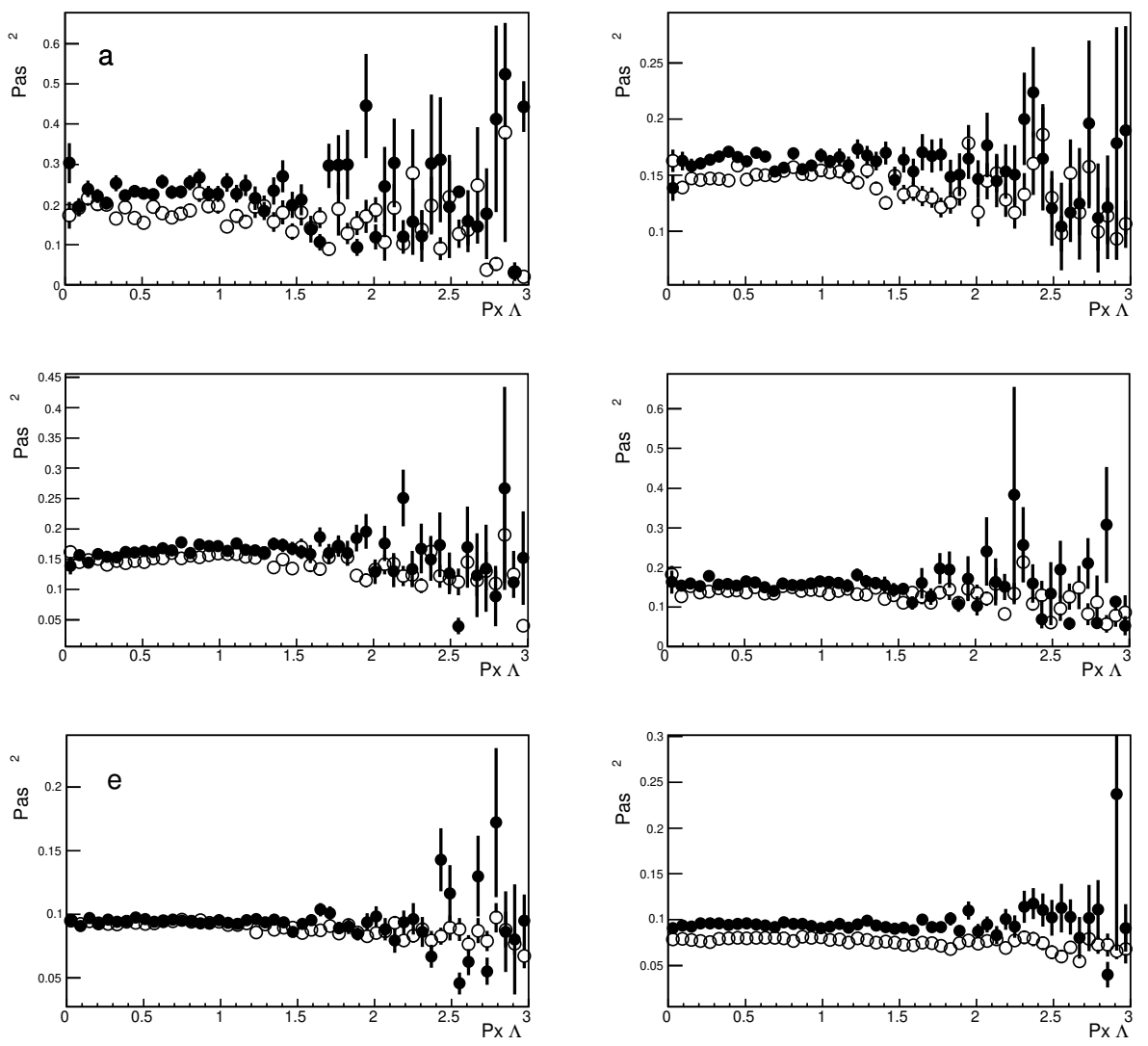

Fig. 35. $<\left(P_{y}^{a s}\right)^{2}>$ as functions of triggered $\Lambda$ momentum for associated particles $-\bar{\Lambda}, K^{0}$, $K^{+}, K^{-}, \pi^{+}$and $\pi^{-}$, figures $a, b, c, d, e$ and $f$, respectively, in $p p$ interactions at $\sqrt{s_{N N}}=25$ $\mathrm{GeV}$. Open and filled symbols are Pythia and FTF calculation results, respectively. 


\section{Puzzles of soft photons pp, pA and AA interactions ${ }^{1}$}

Over three decades there has been no comprehensive understanding of the mechanism of soft photons (energy smaller $50 \mathrm{MeV}$ ) formation. Experimental data indicate an excess of their yield in hadron and nuclear interactions in comparison with calculations performed theoretically. In JINR, in connection with the building of a new accelerator complex NICA, it has become possible to carry out such studies in pp, pA and AA interactions at energies up to $25 \mathrm{AGeV}$. We prepared the extensive physical program for soft photons that covers the wide region of investigations in high energy physics. To carry out this program our group develops the conception of an electromagnetic calorimeter on type "shashlik" based on gadolinium-gallium garnet (GaGG) crystals, which have significantly lower the threshold for registration of photons. The first tests of electromagnetic calorimeters manufactured at JINR on the basis of the GaGG and a composite of tungsten and copper confirm that choice.

PACS: 12.39.-x, 12.40.-y

Over more than three decades, a question about the mysterious origin and properties of soft photons (SP), has been lacking a comprehensive understanding. A few experimental groups found out an increased yield of photons in the region of low transverse momentum, $p_{T}$, smaller than $50 \mathrm{MeV} / c$ [304-312]. These photons are not the decay products of short-living particles (including resonances).

According to QCD, at high energies, $q \bar{q}, q g$ and $g g$ interactions lead to the emission of photons called direct photons. In spite of the fact that the majority of photons in high energy interactions comes from decays of secondary hadrons (neutral mesons and others), the direct photons present a unique opportunity to study the soft gluon component of a nucleon and a stage of hadronisation. Direct photons interact with the surrounding medium only electromagnetically as opposed to the strong interaction of hadrons. So SP keep more information about the medium at all stages of the interaction. This is especially valuable, along with the information about the secondary hadrons.

The first convincing experiment on the detection SP has been carried out at the Big European Bubble Chamber (BEBC) [304]. The study of SP production in $K^{+} p$ interactions at $70 \mathrm{GeV} / c$ beams of $K^{+}$-mesons after the subtraction of all known hadron decays indicated their excess compared to what was expected from QED inner bremsstrahlung.

The next series of experiments have been conducted at higher energies [305-309]. The excess of the SP yield was fourfold or even higher, eightfold. The last European experiment has been realised at the SPS accelerator, CERN by the DELPHI Collaboration [310]. The yield of SP as a function of the neutral pion number turned out to be completely unexpected [311]. The excess of the inner bremsstrahlung rates over the predicted ones as a function of the neutral multiplicity in the quark jet turned out to be seventeen-fold for the largest recorded number of pions $N_{\text {neu }}=6$. The muon bremsstrahlung photons in the reaction $e^{+} e^{-} \rightarrow \mathrm{Z}^{0} \rightarrow \mu^{+} \mu^{-}$has demonstrated a good agreement of the observed photon rate with predictions from QED for the muon inner bremsstrahlung [312].

Our BGO calorimeter has been installed at the NIS-GIBS setup at an angle of $16^{\circ}$ relative to the beam direction, registering the energy release of gamma quanta. The

\footnotetext{
${ }^{1}$ This section is written by E.Kokoulina (E-mail:kokoulina@jinr.ru) and V.A. Nikitin (E-mail: nikitin@jinr.ru).
} 
Monte Carlo simulation has also been carried out in the conditions of the last assembly and with the beam energy 3.5 A GeV. Spectra of photon energy release in deuteriumcarbon and lithium-carbon (see [313]) interactions have been obtained. In the region of energy release below $50 \mathrm{MeV}$, a noticeable excess over the Monte-Carlo simulation is observed.

There are a few phenomenological models which have been worked out to describe the SP spectra [314-317]. The most attractive among them is the model of the cold quark-gluon plasma of L. Van Hove [314]. But until now, a complete comprehensive understanding of the nature of the anomalous yield of SP has not been achieved.

\subsection{The scientific program of SP study}

In accordance of the gluon dominance model (GDM) design [317,318] multiparticle production is described as the convolution of two stages. The first stage is described in QCD as a quark-gluon cascade. For the second one, the phenomenological scheme of hadronization is applied. GDM evidences that the sources of secondary particles are gluons that we call active, and an abundance of soft gluons can be the sources of SP. They are picked up by newly born quarks with a subsequent dropping of energy by emission of SP: $g+q \rightarrow \gamma+q$ or $q+\bar{q} \rightarrow \gamma$. At that valence quarks are staying in the leading particles. Our estimations of the emission region of SP (its linear size) for $p p \rightarrow$ hadrons + photons at U-70 in case of a near-equilibrium state at using the black body radiation exceeds the typical hadronisation region $(1 \mathrm{fm})$ and reaches a value of about $4-6 \mathrm{fm}[318]$. We consider the soft gluon component of protons is relevant for the understanding of the spin structure of nucleons.

The formation of the pionic (Bose-Einstein) condensate in the region of high total multiplicity $(\mathrm{N}\rangle\rangle\langle N\rangle, N=N_{c h}+N_{0},\langle N\rangle$ - the average multiplicity of charged and neutral pions) can be related with the exceeding yield of SP [315]. The growth of the scaled variance $\omega^{0}=\left(\left\langle N_{0}^{2}>-\left\langle N_{0}\right\rangle^{2}\right) /<N_{0}>\right.$ will be the signal of pionic condensate formation [319].

The increasing yield of photons at low energy gives us the opportunity to calculate two particle correlations of direct photons. Similar results were obtained by the WA98 Collaboration [320]. The deviation from theoretical predictions is at the level of a fraction of a percent.

The next item of our scientific program appeared thanks to the RHIC experiment, namely with using the variable flow or $v_{2}$. There is an interesting prediction in [321] about the growth of $v_{2}$ in the region of small $p_{T}$ of photons. This dependence can be the evidence of a coherent SP emission. Our program proposes to test this behaviour.

The scientist from the US Cheuk-Yin Wong [316] develops an intriguing model of open string QED mesons to explain and describe the $p_{T}$-spectrum of SP. Wong believes that since $q$ and $\bar{q}$ cannot be isolated, the intrinsic motion of the $q \bar{q}$ system in its lowestenergy state lies predominantly in $1+1$ dimensions as in the open string with $q$ and $\bar{q}$ at its two ends. Extrapolating into the $q \bar{q}$ QED sector in which $q$ and $\bar{q}$ interact with the QED interaction, he finds an open string QED meson state at 17.9 $\pm 1.5 \mathrm{MeV}$ and QED meson state at $36.4 \pm 3.8 \mathrm{MeV}$. These predicted masses of the isoscalar and isovector QED mesons are close to the masses of the hypothetical X17 [322] and E38 [323] particles 
observed recently, making them good candidates for these particles. This hypothesis has generated a great deal interest [324].

The decay of these particles can show up as an excess of $e^{+} e^{-}$and $\gamma \gamma$ pairs in the SP phenomenon [316]. Wang shows that an astrophysical object consisting of a large assembly of QED mesons such as the X17 particle with a mass $m_{X}=17 \mathrm{MeV}$ will be an electron-positron and gamma-ray emitters. If the temperature of such an assembly is low, it can form a Bose-Einstein condensate. Such assemblies of QED mesons present themselves as good candidates as $e^{+} e^{-}$emitters, gamma-ray emitters, or a part of the primordial cold dark matter.

\subsection{The preparation to experimental SP study}

To carry out our scientific program we have to manufacture a space saver electromagnetic calorimeter (ECal) with the capacity of low energy gamma quanta detection . As it is known the production of homogeneous crystalline ECal's is expensive. Usually physicists prefer making heterogeneous assemblies [325]. They are cheaper and at that possess satisfactory properties. Our activity was aimed at testing of two types of ECal's. The "spaghetti" type was the first prototype that we produced and irradiated with photon beams in Germany [313].

We substituted light scintillator material for a very dense (heavy) crystal having a high specific light yield [325]. It allowed us to build a device that is capable of maintaining considerable compactness (space-saving of about 30\%). To produce the "spaghetti" type we have chosen a mono-crystal of gadolinium-gallium garnet, $\mathrm{Gd}_{3} \mathrm{Al}_{2} \mathrm{Ga}_{3} \mathrm{O}_{12}$ : $\mathrm{Ce}(\mathrm{GaGG}$ ), as a scintillator and tungsten+copper composite by way of the absorber. We expect the decay time to be $\sim 90 \mathrm{~ns}$; the light yield to be $\sim 45,000-55,000 \mathrm{ph} / \mathrm{MeV}$; an estimated price of $25-35 \$ / \mathrm{cm}^{3}$ of volume, and a good radiation resistance.

GaGG crystal is a fast-acting scintillator, its light yield is 4 times greater than that of BGO crystals. And we can acquire these crystals from the well-known domestic firm "Fomos-Materials". The Monte-Carlo simulation has shown that the necessary energy resolution for SP in the case of the ECal "spaghetti" type turns out lower than when at using the "shashlik" type. See figures in [313]) for an example of a typical view of the ECal "shashlik" type.

We plan to assemble the ECal "shashlik". It is assumed that it will consist or 16 or more of GaGG plates $(100 \times 100 \times 3) \mathrm{mm}^{3}, 15$ plates of 2 -mm-absorber $(\mathrm{W}: \mathrm{Cu}$ composite, 1:19), and its total length will be about $138 \mathrm{~mm}$. GEANT4 simulation shows that the energy resolution of the "shashlik" is considerably better than "spaghetti" type. The dimensions of both assemblies were optimised by the Monte-Carlo simulation for obtaining the required characteristics. The design of the "shashlik" allows expanding it up to any length. We also can exclude the W/Cu absorber from the "shashlik" and then to assemble the homogeneous device for the detection of $\mathrm{MeV}$ photons if the energy resolution of the ECal will not be of a good enough quality.

We would like to thank all participants of SP study activity for a long years.and also the leadership of our laboratory for significant support. 


\section{Hadron formation effects in heavy ion collisions ${ }^{1}$}

The space-time picture of hadron formation in high energy processes with nuclear targets is still poorly known. It is suggested to test different models of hadron formation by using collisions of heavy ions. Results of microscopic transport calculations of proton and charged pion rapidity and transverse momentum distributions in $\mathrm{C}+\mathrm{C}$ and $\mathrm{Ca}+\mathrm{Ca}$ collisions at $\sqrt{s}_{N N}=11 \mathrm{GeV}$ are presented

PACS: 25.75.-q; 25.75.Bh; 25.75.Dw

Hadrons produced in a hard exclusive particle-nucleon collisions emerge first in a form of prehadrons having a reduced transverse size $\sim 1 / \sqrt{Q^{2}}$ where $Q^{2}$ is a hard scale. For exclusive meson electroproduction $e N \rightarrow e^{\prime} N^{\prime} M(M=\pi, \rho)$, the hard scale is the photon virtuality (cf. Ref. [326]). In the case of large-angle hadronic scattering $h N \rightarrow h^{\prime} N^{\prime}$ with $-t \simeq-u \simeq s / 2$, the hard scale is given by the momentum transfer, i.e. $Q^{2}=-t$, (cf. Ref. [327] and refs. therein). The prehadrons, called also the point-like configurations (PLCs), interact with nucleons with reduced strength which is known as the color transparency (CT) phenomenon, see Ref. [328] for the most recent review of CT.

In inclusive production channels of the $N N$ collisions, there is no fixed hard scale, even at large $\sqrt{s}$. Thus, formation of PLCs is questionable here. However, the hard scale will fluctuate event-by-event and one can expect that in average the transverse size of the outgoing hadrons will be still reduced. This motivates inclusion of the hadron formation effects in microscopic transport models for high-energy heavy ion collisions, such as UrQMD [329], HSD [330], GiBUU [331]. ${ }^{2}$

Hadron formation studies in the current fragmentation region [332] based on the Deep Inelastic Scattering (DIS) data from HERMES and EMC concluded the linear increase of the prehadron-nucleon cross section with time (or length traveled by a prehadron), in agreement with the quantum diffusion model (QDM) [137]. In the QDM, the prehadron is converted to the 'normal' hadron after passing the distance called formation (or coherence) length

$$
l_{h} \simeq \frac{2 p_{h}}{\left|M_{h}^{2}-M_{h^{\prime}}^{2}\right|} \sim 0.4-0.6(\mathrm{fm} / \mathrm{GeV}) \cdot p_{h}(\mathrm{GeV})
$$

where $p_{h}$ is the momentum of hadron $h, M_{h}$ is the mass of the hadron $h$, and $M_{h^{\prime}}$ is roughly the mass of the closest radially excited state $h^{\prime}$. Thus, based on the QDM one can estimate that particles with momenta above $\sim 10 \mathrm{GeV}$ leave the nucleus almost without interactions. On the other hand, the analyses of E665 data on the low-energy $(E<10 \mathrm{MeV})$ neutron production in the $470 \mathrm{GeV}$ muon DIS on $\mathrm{Pb}$ target $[333,334]$ favor a surprisingly low cutoff value of the momentum $\sim 1 \mathrm{GeV}$ above which the prehadrons do not interact in the nucleus. This can not be explained by the QDM or any other existing model of hadron formation and calls for the new experiments on hadron formation in the target fragmentation region, e.g. in virtual photon - nucleus collisions

\footnotetext{
${ }^{1}$ This section is written by A.B. Larionov; E-mail: larionov@theor.jinr.ru

${ }^{2}$ Based on the CT concept, also the incoming nucleons are transversely 'squeezed' if they are in the initial state of a hard collision. This effect is totally neglected in transport models.
} 
at the future Electron-Ion Collider (EIC) or in ultraperipheral heavy ion collisions at the LHC and RHIC [334]. The physics of target fragmentation is one of perspectives for the experimental program of the EIC [335].

Another opportunity to study hadron formation effects is offered by heavy ion collisions at NICA-SPD regime $\left(\sqrt{s}_{N N} \sim 10 \mathrm{GeV}\right)$. Here, the stopped nucleon in the $N N$ center-of-mass system (c.m.s.) has $x_{F}=m_{N} / \sqrt{s}_{N N} \sim 0.1$. Therefore, slow nucleons in the $N N$ c.m.s. represent a mixture of the current $\left(x_{F} \ll 1\right)$ and target $\left(x_{F} \lesssim 1\right)$ fragmentation products. This paper addresses the sensitivity of heavy ion collision observables to the different treatments of hadron formation. The calculations are performed within the Giessen Boltzmann-Uehling-Uhlenbeck (GiBUU) model.

In sec. 14.1, the GiBUU model is briefly described with a focus on hadron formation. Sec. 14.2 contains the results of numerical calculations of the the rapidity- and $p_{t}$ spectra of protons and pions in $\mathrm{C}+\mathrm{C}$ and $\mathrm{Ca}+\mathrm{Ca}$ collisions at $\sqrt{s}_{N N}=11 \mathrm{GeV}$. The summary and estimation of rates at NICA-SPD are given in sec. 14.3.

\subsection{The model}

The GiBUU model (see detailed description in ref. [331]) solves the system of kinetic equations for the baryons $\left(N, N^{*}, \Delta, \Lambda, \Sigma, \ldots\right)$, corresponding antibaryons $\left(\bar{N}, \bar{N}^{*}, \bar{\Delta}\right.$, $\bar{\Lambda}, \bar{\Sigma}, \ldots)$, and mesons $(\pi, K, \ldots)$. The kinetic equations are coupled via collision integrals and mean field potentials. The latter are determined self-consistently, i.e. they depend on the actual particle distributions in the six-dimensional phase space of position and momentum. The distribution function is projected onto the set of point-like test particles. Kinetic equation is then solved if the test particle positions and momenta propagate in time according to the Hamiltonian equations of motion between collisions. ${ }^{1}$ The two test particles experience a two-body collision during the time interval $[t-\Delta t / 2 ; t+\Delta t / 2]$ if they approach their minimal distance $d$ in position space during this time interval and if $d<\sqrt{\sigma_{\text {tot }} / \pi}$. Here, $\Delta t$ is the time step in the computational frame that is chosen to be the nucleus-nucleus c.m.s. and $\sigma_{\text {tot }}$ is the total interaction cross section of colliding particles.

The model also includes three-body collision processes $\pi N N \rightarrow N N$ and $\Delta N N \rightarrow$ $N N N$ which are simulated by the direct calculation of the three-body absorption rate. Unstable resonances experience decays with a probability $P=\Gamma \Delta t$ where $\Gamma$ is the total decay width of a resonance. The final states of the two- and three-body collisions and resonance decays are sampled by the Monte-Carlo method according to the partial channel cross sections, widths and angular distributions. Pauli blocking is taken into account for the final state nucleons.

For low-energetic elementary binary collisions, the resonance model complemented with empirical background cross sections (e.g. $s$-wave direct pion production $N N \rightarrow$ $N N \pi$ ) is implemented. For such collisions, it is assumed that all produced particles are formed immediately and interact with usual hadronic cross sections.

\footnotetext{
${ }^{1}$ In the present calculations we disregard mean field potentials. Thus, the test particles propagate along straight-line trajectories.
} 
High-energy elementary binary collisions are simulated by the PYTHIA and FRITIOF models. (The latter is applied for antibaryon-baryon collisions only.) The corresponding transition values of $\sqrt{s}$ for meson-baryon, baryon-baryon, and antibaryon-baryon collisions are $2.2,4.0$ and $2.38 \mathrm{GeV}$. Note that in the narrow $\sqrt{s}$ region $\pm(0.1-0.2) \mathrm{GeV}$ centered at the transition value, the low- and high-energy events are mixed to ensure a smooth behaviour of the cross sections between the two energy regimes.

The particles produced in a high-energy binary collision are supposed to be in a prehadronic state. Their effective interaction cross section $\sigma_{\text {eff }}$ with nucleon becomes time-dependent and deviates from the ordinary interaction cross section $\sigma_{0}$. We applied the three different models for the ratio of the effective and ordinary interaction cross sections:

(i) Based on JETSET-production-formation points (see ref. [336], used as default in GiBUU) favored by analysis of hadron attenuation at HERMES and EMC [332]:

$$
\sigma_{\text {eff }}(t) / \sigma_{0}=X_{0}+\left(1-X_{0}\right) \frac{t-t_{\text {prod }}}{t_{\text {form }}-t_{\text {prod }}}
$$

where $X_{0}=r_{\text {lead }} a / Q^{2}, a=1 \mathrm{GeV}^{2}, r_{\text {lead }}$ is the ratio of the number of leading quarks to the total number of quarks in the prehadron. $Q^{2}$ is defined for the hard $2 \rightarrow 2$ subprocess by the PYTHIA variable VINT(52). By default, it gives $Q^{2} \sim p_{\perp}^{2}$ where $p_{\perp}$ is the transverse momentum of the hard scattering (see PYTHIA 6.4 manual [337] for detail).

(ii) QDM [137]:

$$
\sigma_{\text {eff }}(t) / \sigma_{0}=X_{0}+\left(1-X_{0}\right) \frac{c\left(t-t_{\text {hard }}\right)}{l_{h}} .
$$

Here we set $X_{0}=0$ for simplicity. $t_{\text {hard }}$ is the time moment of the collision in GiBUU calculation.

(iii) Momentum cutoff:

$$
\sigma_{\text {eff }} / \sigma_{0}=\Theta\left(p_{\text {cut }}-p_{h}\right), \quad p_{\text {cut }} \sim 1-2 \mathrm{GeV} / \mathrm{c}
$$

Some comments are in order with regard to the Lorentz covariance. In principle, all three above models are explicitly non-covariant. Thus, one needs to specify the frame in which they are applied. In the case of models (i) and (ii) the non-covariance, however, should largely cancel-out in the ratios of time- or length differences. The model (iii) is most problematic: it is clear that the result will certainly depend on the frame in which the particle momentum is calculated. Note that originally Eq.(56) has been suggested for the target nucleus rest frame [334] where DIS is calculated in GiBUU. In contrast, in the present work, the nucleus-nucleus c.m.s. is used as the calculational frame. The problem related to the choice of the calculational frame is beyond the scope of this work and should be addressed in future studies. 


\subsection{Numerical results}

The calculations were performed for the systems $\mathrm{C}+\mathrm{C}$ and $\mathrm{Ca}+\mathrm{Ca}$ at $\sqrt{s}_{N N}=11 \mathrm{GeV}$. The maximum impact parameter was set to $8 \mathrm{fm}$ for $\mathrm{C}+\mathrm{C}$ and to $11 \mathrm{fm}$ for $\mathrm{Ca}+\mathrm{Ca}$ that corresponds to the minimum bias trigger. The time evolution of the colliding system was calculated until the maximum time $30 \mathrm{fm} / \mathrm{c}$ with the time step $\Delta t=0.2 \mathrm{fm} / \mathrm{c}$. The total accumulated statistics is $2 \cdot 10^{6}\left(1 \cdot 10^{6}\right)$ nucleus-nucleus collision events for $\mathrm{C}+\mathrm{C}$ $(\mathrm{Ca}+\mathrm{Ca})$.

Fig. 36 shows the rapidity spectra of protons and pions. Note that we do not distinguish free protons from those bound in nuclear clusters. This explains the maxima in the proton rapidity spectra at the target and projectile rapidities. Calculation without hadron formation gives the largest production at midrapidity $(y=0)$. Hadron formation leads to the reduced FSI of the outgoing hadrons. Hence, the produced protons lose less longitudinal momentum which results in the depletion of the proton spectrum at midrapidity. Since less FSI implies less inelastic production by secondary protons, also the pion spectrum gets depleted at midrapidity in calculations with hadron formation. As expected, the depletion is stronger for heavier system and for calculations with more restrictions on FSI $\left(p_{\text {cut }}=1 \mathrm{GeV} / \mathrm{c}\right)$.

Fig. 37 shows the proton and charged pion transverse momentum spectra at midrapidity. The proton $p_{t}$ spectrum becomes softer due to hadron formation. Indeed, protons effectively gain transverse momentum in the rescattering processes while hadron formation suppresses rescattering. Pion $p_{t}$ spectrum is influenced by hadron formation only very weakly. In the collisions of heavier nuclei [338] the hadron formation effect becomes more pronounced, especially for central events.

\subsection{Summary and conclusions}

The purpose of this work was to clarify whether heavy ion collisions can be used to test various models of hadron formation. To this end, the GiBUU transport model was used that includes the three optional models of hadron formation. The first two models, (i) and (ii) [Eqs. (54),(55)] are based on the CT mechanism and differ only in the details of the hadron formation length. Both these models were successful in the description of hadron attenuation in DIS reactions. The third model (iii) [Eq.(56] assumes that the prehadrons with momenta above $1-2 \mathrm{GeV} / \mathrm{c}$ do not interact with nucleons which is in stark contrast with widely accepted momentum dependence of the hadron formation length, Eq.(53). This model was originally adopted to describe the low-energy neutron production induced by the muon DIS on nuclear targets [334].

The GiBUU model calculations were performed for inclusive proton and charged pion production in $\mathrm{C}+\mathrm{C}$ and $\mathrm{Ca}+\mathrm{Ca}$ at $\sqrt{s}_{N N}=11 \mathrm{GeV}$. In the $\mathrm{C}+\mathrm{C}$ system, the secondary rescattering effects are minimal and the reaction process is governed by first-chance $N N$ collisions. The $\mathrm{Ca}+\mathrm{Ca}$ system is more suitable for the study of FSI effects. Proton and charged pion rapidity distributions and proton $p_{t}$ distributions are strongly sensitive to the hadron formation effects. More restrictions on FSI of prehadronic states leads to less production at midrapidity and to softer proton $p_{t}$ spectra. 
In the $\mathrm{C}+\mathrm{C}$ system the proton and $\pi^{ \pm}$production cross sections in the c.m.s. rapidity window $|y|<0.5$ are about $0.4 \mathrm{~b}$ and $3 \mathrm{~b}$, respectively. In the $\mathrm{Ca}+\mathrm{Ca}$ system the corresponding cross sections are about one order of magnitude higher. Thus, the measurements of the proton and charged pion spectra seems easily possible at the first stage of NICA-SPD operation $\left(L=10^{25} \mathrm{~cm}^{-2} \mathrm{~s}^{-1}\right)$ with the production rate of about 1-10 events per second (without efficiency and acceptance corrections). The accuracy needed to separate different models of hadron formation should be better than $\sim 10 \%$ for the production cross sections at midrapidity which is reachable within few hours of beam time. 

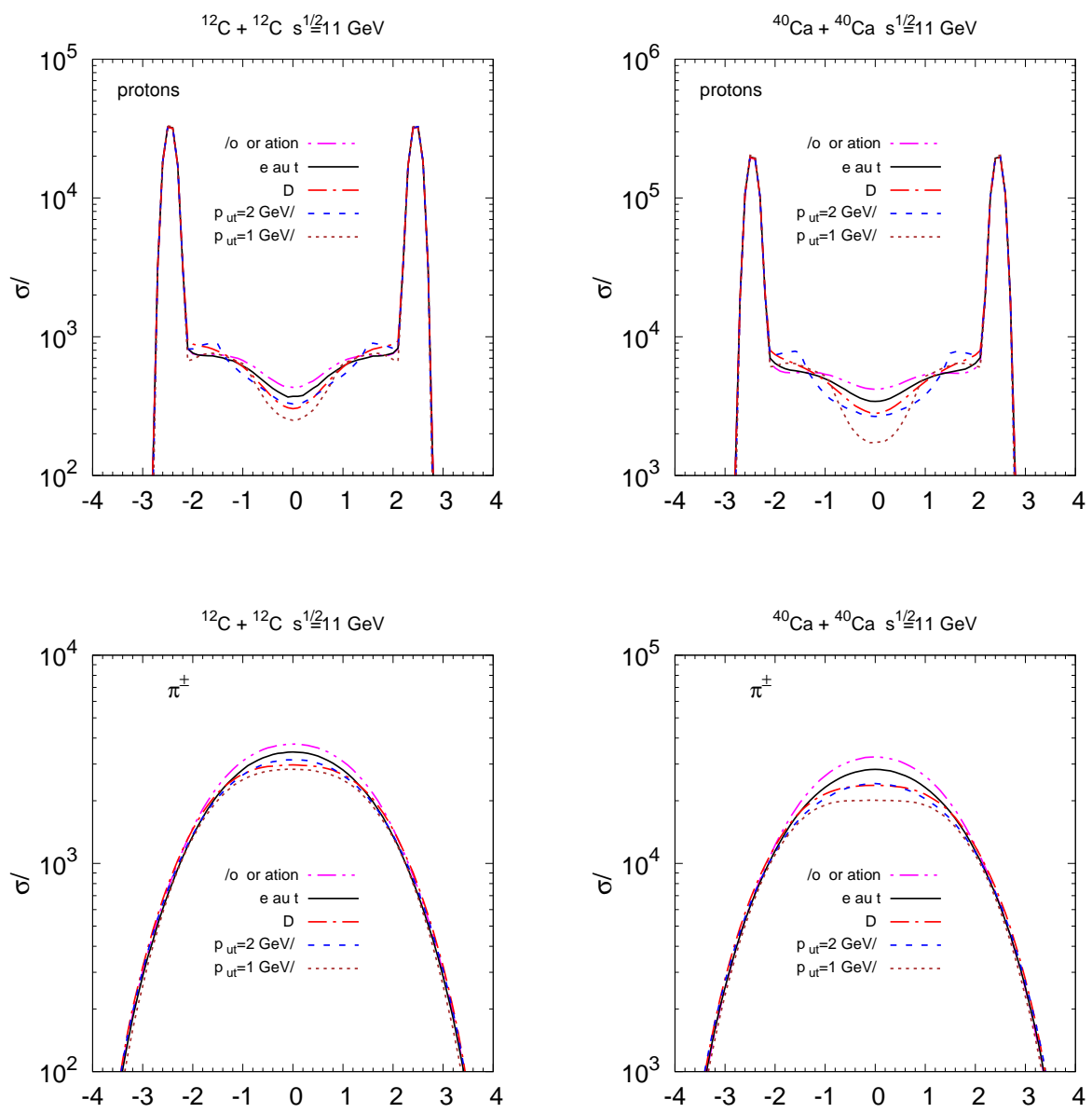

Fig. 36. Proton and charged pion rapidity distributions in $\mathrm{C}+\mathrm{C}$ and $\mathrm{Ca}+\mathrm{Ca}$ at $\sqrt{s}_{N N}=11$ $\mathrm{GeV}$ as indicated. Dot-dot-dashed (magenta) line - calculation without formation (immediately formed hadrons), Solid (black) line - default formation (i). Dot-dashed (red) line - QDM model (ii). Dashed (blue) line $-p_{\text {cut }}=2 \mathrm{GeV} / \mathrm{c}$ (iii). Dotted (brown) line $-p_{\text {cut }}=1 \mathrm{GeV} / \mathrm{c}$ (iii). Here (i), (ii) and (iii) refer to the models defined in the text, see Eqs.(54),(55),(56), respectively. 

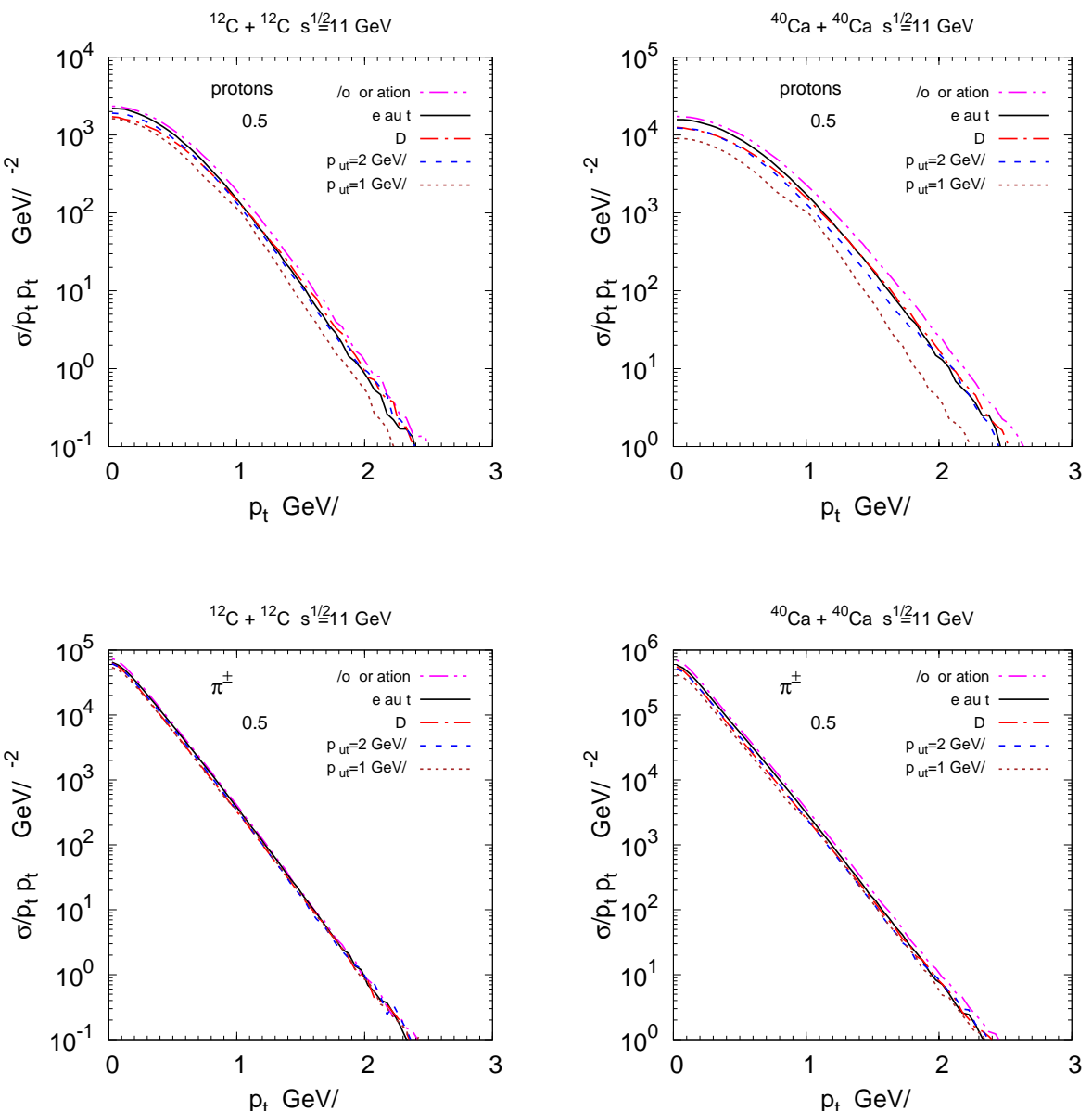

Fig. 37. Proton and charged pion transverse momentum distributions in $\mathrm{C}+\mathrm{C}$ and $\mathrm{Ca}+\mathrm{Ca}$ at $\sqrt{s}_{N N}=11 \mathrm{GeV}$ as indicated. C.m.s. rapidity cut $|y|<0.5$ is applied. Line notation is the same as in Fig. 36 . 


\section{Measurement of characteristics of the processes of pair production of polarized tau leptons in the SPD experiment. ${ }^{1}$}

It is proposed to use the Drell-Yan process with pair production of $\tau$ leptons to measure the parameters of polarized parton distribution functions of a proton at the NICA collider in the SPD experiment. To determine the polarization of tau leptons, we propose to use decays of $\tau$ leptons into a single charged pi-meson and neutrino. To parameterize the polarization state of $\tau$ leptons, it is proposed to use the energy of single $\pi$-mesons.

PACS: 24.10.Lx, 13.85.Ni, 14.20.-c

The probability of the process with a particular parton is described by the function, which is dependent on the fraction of the total momentum of the proton carried by that parton and the momentum transfer scale. These functions are known as parton distribution functions (PDF) and contain information about the internal structure of corresponding nucleons $[339,340]$. Hence, for a consistent description of processes with polarized nucleons in a wide energy range and the most complete description of the proton structure a generalized parton model was developed [341-343], which consist of 8 specialized parton distribution functions. These functions are: the distribution of the parton density in unpolarized nucleon (Density); distribution of the longitudinal polarization of quarks in the longitudinally polarized nucleon (Helicity); distribution of transverse polarization of quarks in transversely polarized nucleon (Transversity); the correlation between the transverse polarization of a nucleon and the transverse momentum of unpolarized quarks (Sivers); the correlation between the transverse polarization of a nucleon and the longitudinal polarization of quarks (Worm-gear-T); the distribution of the transverse momenta of quarks in unpolarized nucleon (Boer-Mulders); the correlation between longitudinal polarization of nucleon and the transverse momenta of quarks (Worm-gear-L); the distribution of the transverse momenta of quarks in transversely polarized nucleon (Pretzelosity).

At the current level of our knowledge of the nucleon structure, parton distribution functions cannot be obtained analytically and have to be measured experimentally. The deep inelastic scattering (DIS,SIDIS) and the Drell-Yan (DY) processes are typically used for the measurements of PDFs [344-349,349-356].

In the Drell-Yan processes, the annihilation of a quark and antiquark, from colliding nucleons, through an intermediate photon or Z-boson leads to the production of a leptonantilepton pair $[349,357]$. The matrix element of the Drell - Yang process consists of a convolution of two tensors $[349,358,359]$. The first one is responsible for the hadron part with initial partons and includes a dependence on the polarized parton distribution functions. The second tensor corresponds to the lepton part of the process. Such a simple structure and the fact that Drell-Yan process is one of the cleanest hard hadron-hadron scattering processes makes it a very convenient tool for studying PDFs.

The conventional way for studying parton distribution functions via Drell-Yan process is to use processes with the production of electron-positron (and muon anti-muon) pairs $[349,354,356]$, because in this case the mass of leptons can be neglected and one can

\footnotetext{
${ }^{1}$ This section is written by A. Aleshko, E. Boos (E-mail: boos@theory.sinp.msu.ru), V. Bunichev (Email: bunichev@theory.sinp.msu.ru)
} 
consider them as having some definite helicity. Within this approach angular parameters, which define the spatial orientation of the momenta of leptons relative to the momenta of colliding nucleons, are used as parameters describing the polarization state of the lepton pair. Then one can measure azimuthal asymmetries and extract particular structure functions from them.

In the case of $\tau$ production, this approach will work only at very high energies. At lower energies, however, the mass of tau cannot be neglected. Massive particles do not have definite helicity. Therefore, for $\tau$ leptons, the angular parameters as spin-sensitive variables can be used only at collision energy much greater than the mass of the $\tau$ lepton and are not suitable for energies comparable to its mass. Consequently, we need a more suitable parameterization of the polarization state of $\tau$ lepton.

Our idea to tackle this problem is to utilize the unique decays properties of $\tau$-lepton. Consider the hadronic decay of $\tau$ to a single charged $\pi$-meson and neutrino. Due to the weak nature of this decay and the fact that neutrinos are always left-handed, the energy spectra of $\pi$-meson is strictly correlated with the polarization state of decaying $\tau$ lepton [360-363]. For instance, in the case of mostly right-handed $\tau$ decay, due to the angular momentum conservation law, most of the tau's momentum is transferred to the $\pi$-meson. On the contrary, in the case of mostly left-handed $\tau$, the significant part of its momentum is transferred to the neutrino. Thereby, the energy of single $\pi$-meson is a convenient characteristic of the polarization state of its parent $\tau$. So, to determine the polarization of $\tau$ leptons, we suggest to use hadron decays of $\tau$ leptons to a single charged $\pi$-meson and neutrino. The diagram of the corresponding process is shown in the Figure 38. We propose to use the energy of single $\pi$ meson as variable that parametrize the polarization state of $\tau$ leptons. A key feature of our method is that we do not summarize the $\tau$ lepton polarizations, but keep information about the polarizations of both $\tau$ leptons through the energies of single $\pi$ mesons from $\tau$ decays. Due to the fact that a pair of $\tau$ leptons is produced at the NICA collider near the reaction threshold, depending on the parton momenta, the $\tau$ leptons can be produced almost at rest or have a significant momentum, which is reflected in the decay products of the $\tau$ leptons and makes the process with $\tau$ leptons are very sensitive to the state of the spins and momenta of the initial partons and nucleons.

One of the closest opportunities for testing this approach is the future NICA SPD experiment [364]. Since at the NICA collider a pair of $\tau$ is produced near the reaction threshold, the leptons can be at rest or possess a significant momentum, which affects the $\tau$ decay products and makes this process very sensitive to the polarization states and momenta of the initial hadrons.

To confirm the efficiency of our method, we carried out a detailed numerical simulation. First, using the CompHEP [365] package, we computed the tree level cross-section of the Drell-Yan $\tau$ pair production process. Also, we used the MadGraph5_aMC@NLO package [366] to estimate the cross section of the same process at the next to the leading order in perturbation theory. In both cases we have used the NNPDF3.1 PDF set [367]. As it has been mentioned, the important advantage of the NICA facility is its energy range $\sqrt{s}=10-26 \mathrm{GeV}$, which lies close to the $\tau$ pair production threshold. However, one of the problems arising in calculating the cross-sections at such low energies is the correct choice of the QCD factorization scale. At present, certain ambiguity exists as to how to choose this scale properly. When dealing with Drell-Yan process it is often 


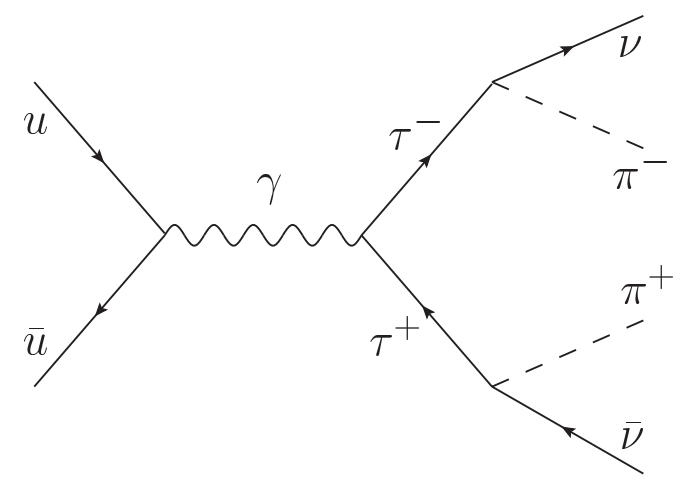

Fig. 38. Drell-Yan process with the pair-production of tau leptons and their subsequent decay into single charged $\pi$-meson and neutrino.

\begin{tabular}{|c|c|c|}
\hline Order & \multicolumn{2}{|c|}{ LO } \\
\hline Scale & cross-section, $\sigma \mathrm{pb}$ & MC error, $\Delta \sigma \mathrm{pb}$ \\
\cline { 2 - 3 }$Q$ & 70.0 & 0.3 \\
$Q / 2$ & 84.8 & 0.4 \\
$E_{T}$ & 75.7 & 0.4 \\
$m_{T}$ & 70.7 & 0.3 \\
\hline \hline Order & \multicolumn{2}{|c|}{ QCD NLO } \\
\hline Scale & cross-section, $\sigma \mathrm{pb}$ & MC error, $\Delta \sigma \mathrm{pb}$ \\
\cline { 2 - 3 }$Q$ & 83.5 & 0.7 \\
$Q / 2$ & 83.8 & 0.7 \\
$E_{T}$ & 108.5 & 0.6 \\
$m_{T}$ & 105.7 & 0.7 \\
\hline
\end{tabular}

Table 5. Cross-sections of the Drell-Yan tau pair production at energy $\sqrt{s}=24 \mathrm{GeV}$ calculated with different choices of factorization scale $\mu_{F}$.

suggested to choose the scale to be of the order of the invariant mass of the lepton pair $Q$, since it is the most obvious characteristic scale of the process. However, the important point given in the aforementioned work is that the choice of the factorization scale $\mu_{F}=Q$ is not always lead to satisfactory results. The problem especially evident at lower energies, which is the case for NICA. In [368] authors suggest using scale $\mu_{F} \sim 0.5 Q$. Other popular choices of the factorization scale include the transverse energy $E_{T}$ and transverse mass $m_{T}$ of the event. The results obtained using different types of scales at energy $\sqrt{s}=24 \mathrm{GeV}$ shown in Table 5 .

To assess the approximate birthrate of corresponding events in NICA conditions, we utilize the known branching ratio of corresponding $\tau$ decay channel: $\operatorname{Br}\left(\tau^{-} \rightarrow \pi^{-} \nu_{\tau}\right)=$ $(10.83 \pm 0.06) \%$ according to [369]. Table 6 contains the estimation of the number of events at two energies, which correspond to the highest proposed luminosities.

The next important step is to demonstate the manifestation of the effect discussed. In order to do so, we have carried out numerical simulation of the full process $p p \rightarrow$ 


$\sqrt{s}=24 \mathrm{GeV}\left(L=1.0 \cdot 10^{32} \mathrm{~cm}^{-2} \mathrm{~s}^{-1}\right)$
\begin{tabular}{|c|c|c|}
\hline Lower cut on $M_{l^{+} l^{-}}, \mathrm{GeV}$ & 3.56 & 4 \\
\hline$\sigma_{p p \rightarrow \tau^{+} \tau^{-}} \cdot B r_{\tau^{-} \rightarrow \pi^{-} \nu_{\tau}} \cdot B r_{\tau^{+} \rightarrow \pi^{+} \overline{\nu_{\tau}}}, \mathrm{pb}$ & 1.00 & 0.71 \\
\hline Approximate number of events per $7000 \mathrm{~h}$ & 2500 & 1800 \\
\hline \hline \multicolumn{3}{|c|}{$\sqrt{s}=26 \mathrm{GeV}\left(L=1.2 \cdot 10^{32} \mathrm{~cm}^{-2} \mathrm{~s}^{-1}\right)$} \\
\hline Lower cut on $M_{l^{+} l^{-}}, \mathrm{GeV}$ & 3.56 & 4 \\
\hline$\sigma_{p p \rightarrow \tau^{+} \tau^{-}} \cdot B r_{\tau^{-} \rightarrow \pi^{-} \nu_{\tau}} \cdot B r_{\tau^{+} \rightarrow \pi^{+}}, \mathrm{pb}$ & 1.22 & 0.88 \\
\hline Approximate number of events per $7000 \mathrm{~h}$ & 3100 & 2200 \\
\hline
\end{tabular}

Table 6. Estimation of the number of events $p p \rightarrow \pi^{-} \nu_{\tau} \pi^{+} \overline{\nu_{\tau}}$ per year $(\sim 7000 \mathrm{~h})$ of data taking, assuming $100 \%$ efficiency of the detector.

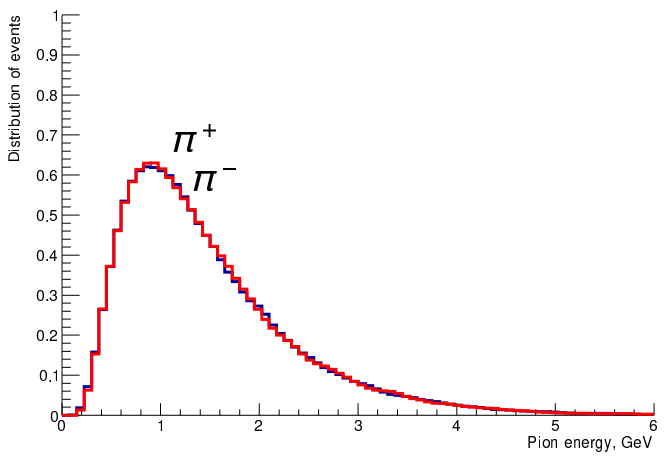

Fig. 39. Energy spectra of $\pi$-mesons in unpolarized case.

$\pi^{-} \nu_{\tau} \pi^{+} \overline{\nu_{\tau}}$ with polarized protons and different polarization of initial partons. Events were generated in CompHEP package. For the simulation of the polarized processes, we have used polarized PDF set NNPDFpol provided by NNPDF collaboration [370]. Currently, only the longitudinally polarized proton version of the PDF is available. Generated events were transferred to Pythia 8 package [371] for parton showering and hadronization. Finally, events were treated by Delphes package [372] for detector simulation and reconstruction.

The energy spectra of pions produced in the unpolarized case can be seen in Figure 39. Both $\pi^{+}$and $\pi^{-}$mesons manifest a similar behaviour. The picture becomes interesting in the case of polarized initial states. The Figure 40 shows the energy spectra of pions produced in the processes with different polarization of initial partons. The first figure corresponds to the case of left-handed quark interacting with right-handed anti-quark while the second one corresponds to the case of right-handed quark interacting with lefthanded anti-quark. As we can see, the energy spectra of the pions are correlating with the polarization states of quarks in the way described in the previous section. Moreover, the clear asymmetry is seen between the energy spectra of $\pi^{+}$and $\pi^{-}$mesons, which can also be utilised as a characteristic of the polarization state of the initial quarks.

The first numerical evaluations carried out show the potential of the new method. In the next step, we will carry out simulations with different initial combinations of 

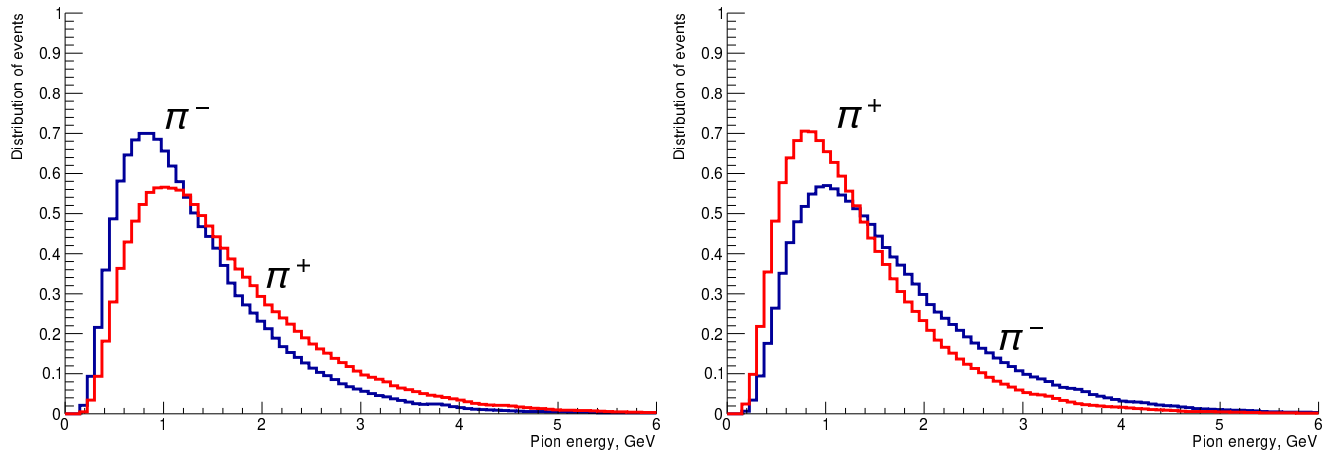

Fig. 40. Energy spectra of $\pi$-mesons produced in processes with different polarization of the initial partons: left picture corresponds to the case of left-handed quark and right-handed antiquark, while right one corresponds to the case of right-handed quark and left-handed anti-quark

longitudinally and transversely polarized protons. Next, we will compile a detailed table of correspondence between the pair distributions of $\pi$ mesons and various combinations of the initial polarizations of protons and their constituent quarks.

Following this approach one can determine the polarization of interacting partons by measuring energies of $\pi$-mesons produced in corresponding decays of $\tau$-leptons. Considering the fact that pions are rather easy detectable [373], the proposed approach is potentially very convenient and powerful tool for studying nuclons PDF via tau production in polarised Drell-Yan process. 


\section{On Measuring Antiproton-Production Cross Sections for Dark Matter Search $^{1}$}

Firm interpretation of the recent results from the AMS-02 and PAMELA spectrometers, regarding the antiproton yield in $p$ - $p$ and $p-d$ collisions, has been hindered by uncertainties in production cross section, angular, and momentum spectra of the produced antiprotons. The proposed measurements of antiproton yield at the planned SPD experiment at the NICA collider could significantly contribute to enhancing the situation in favor of the search for dark matter WIMPs.

PACS: 95.35.+d, 95.55.Vj, 96.50.sb

The dark matter (DM) problem is a long-standing puzzle in modern cosmology. Even though DM is known to make more than $26 \%$ of the total energy-matter content of the Universe [374], we still have no confirmed conclusions about its identity. Evidence of $\mathrm{DM}$ is abundant and diverse, but mainly gravitational. The effects of DM have been observed in the rotation curves of galaxies, the mass discrepancy of galaxy clusters, and the lens-less gravitational lensing, among others [375]. Perhaps most of our knowledge about DM properties was a product of the Bullet Cluster event, where two galaxy clusters collided with one another. Astronomical observations and lensing analyses of the event assured astronomers that DM particles are only weakly interacting [376]. Once merely a tentative hypothesis, this has become widely accepted. Consequently, among many DM candidates, Weakly Interacting Massive Particles (WIMPs) became the most favored [377].

There are several search approaches applied in the case of DM. Each of them has an underlying paradigm. The main three approaches are collider searches, direct detection, and indirect detection. Collider searches are based on the hypothesis that DM particles can be pair-produced in collisions of Standard-Model (SM) particles, either directly or via a mediator. Direct detection experiments try to measure the recoil energy of DM particles colliding with SM nuclei. Indirect detection seeks to prove the hypothesis that DM particles decay and pair-annihilate producing SM particles as final products [377]. Thus, astrophysical searches try to accurately measure rare fluxes in Cosmic Rays (CRs), in order to detect a secondary anomalous signature of DM. To identify an anomalous signal, it is crucial to first subtract other ordinary astrophysical sources. Secondary CR fluxes are produced in collisions of primary CRs on Inter-Stellar Medium (ISM). In particular, secondary antiprotons are produced in proton-nucleus, nucleus-proton, and nucleus-nucleus collisions.

During the last two decades, two satellite-borne experiments, namely, PAMELA [378] and AMS-02 [379], have measured the secondary antiproton flux and antiproton-to-proton ratio with unprecedented accuracy, covering a wide energy range. The AMS-02 experiment has measured antiproton-to-proton ratio with an accuracy higher than $95 \%$ for kinetic energies from 1 to $450 \mathrm{GeV}$ [380]. However, we still cannot come to firm conclusions about any exotic signal because the AMS-02 measurements are still surrounded with several sources of uncertainties as shown in Fig.41. These sources stem from uncertainties on: (i) the primary spectra slopes at high energies, (ii) the solar modulation

\footnotetext{
${ }^{1}$ This section is written by R. El-Kholy; E-mail: relkholy@sci.cu.edu.eg.
} 
at lower energies, (iii) the propagation parameters in the galactic environment, and (iv) the antiproton-production cross sections. The first two can be relatively reduced through new AMS-02 measurements. The third can be minimized via parameter variation. However, the last of these sources is the most significant and ranges between $20 \%$ and $50 \%$, depending on the energy [381].

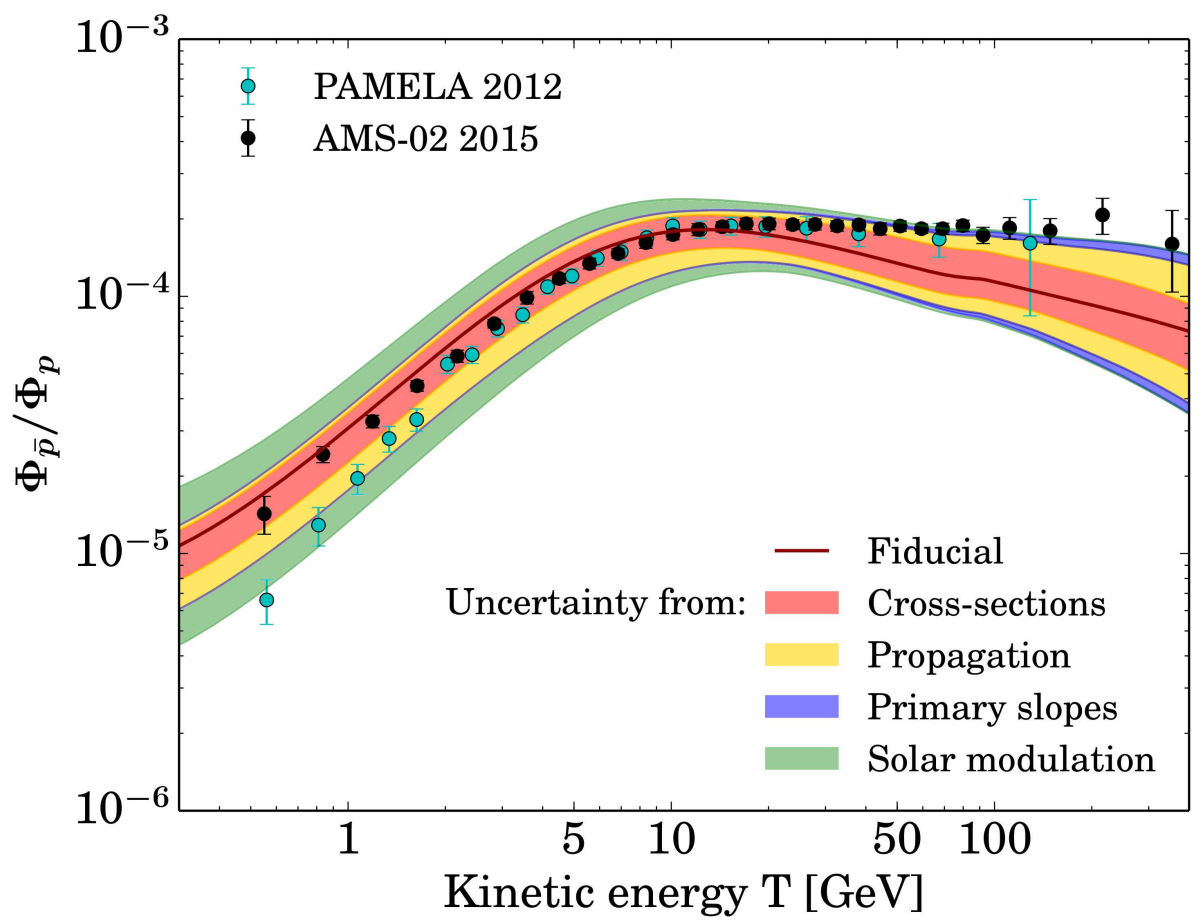

Fig. 41. The combined total uncertainty on the predicted secondary $\bar{p} / p$ ratio superimposed to the PAMELA and the AMS-02 data. Each envelope accounts for uncertainties enclosed in it; such that the green band is the total band of all four sources of uncertainty [381].

In addition to the scarcity of cross-section measurements of antiproton-production, most available datasets date back to before 1980, and did not account for hyperon decay to antiprotons [382]. Thus raising the need for new measurements of antiproton-production cross sections. The kinematic phase-space coverage required to catch up to the AMS-02 measurements has been outlined [383]. Here, we affirm the capability of the Spin Physics Detector (SPD), planned at the NICA collider currently under construction at JINR, to make a sizable contribution to the desired measurements [384,385].

16.1. Antiproton Production Cross Sections 
The contribution of each interaction channel of primary CRs with ISM to the production of secondary antiprotons depends on the abundances of different nuclei in both CRs and ISM. Based on the abundances outlined in [384], the most dominant channel of antiproton production would be proton-proton collisions, followed by proton-helium, and proton-deuteron collisions, respectively. Other nucleus-nucleus collisions have a negligible contribution. In addition to different production channels, there are also different production mechanisms. The dominant mechanism is the direct production. However, antiprotons are also produced via decay of anti-baryons; namely, antineutrons, and the $\bar{\Lambda}$ and $\bar{\Sigma}^{-}$hyperons. The overall antiproton-production cross section can be expressed as $[386]$

$$
\sigma_{\bar{p}}=\sigma_{\bar{p}}^{0}\left(2+\Delta_{\mathrm{IS}}+2 \Delta_{\Lambda}\right)
$$

where $\Delta_{\text {IS }}=\sigma_{\bar{n}}^{0} / \sigma_{\bar{p}}^{0}-1$ is the isospin enhancement factor of antineutron direct production, and $\Delta_{\Lambda}=\sigma_{\bar{p}}^{\Lambda} / \sigma_{\bar{p}}^{0}$ is the hyperon factor, assuming $\sigma_{\bar{n}}^{\Lambda}=\sigma_{\bar{p}}^{\Lambda}$.

The currently-available data on proton-proton collisions is extremely scarce. It is graphically summarized in Fig.42, in terms of transverse momentum, $p_{T}$, and the radialscaling variable, $x_{R}$, which is given by [382]

$$
x_{R}=\frac{E_{\bar{p}}^{*}}{E_{\bar{p} \cdot \max }^{*}},
$$

where $E_{\bar{p}}^{*}$ is the antiproton energy, and $E_{\bar{p} \text {.max }}^{*}$ is the maximal energy it can acquire, both in the CM frame. The maximal antiproton energy can be obtained by [382]

$$
E_{\bar{p} \cdot \max }^{*}=\frac{s-8 m_{p}^{2}}{2 \sqrt{s}} .
$$

Moreover, most of these datasets date back to before 1980, and thus do not include a hyperon-decay feed-down. As for datasets on other production channels, they are essentially non-existent. The first ever proton-helium dataset has been released by the LHCb collaboration in 2018 [387].

In addition to measuring the cross sections of direct antiproton production, it is necessary to accurately estimate the hyperon factor, $\Delta_{\Lambda}$, as well. The hyperon factor can be expressed as [386]

$$
\Delta_{\Lambda}=\frac{\bar{\Lambda}}{\bar{p}} \times \mathrm{BF}_{\bar{\Lambda} \rightarrow \bar{p} \pi^{+}}+\frac{\bar{\Sigma}^{-}}{\bar{p}} \times \mathrm{BF}_{\bar{\Sigma}^{-} \rightarrow \bar{p} \pi^{0}},
$$

where $\bar{\Lambda} / \bar{p}$ and $\bar{\Sigma}^{-} / \bar{p}$ are the hyperon to promptly-produced antiproton ratios, and $\mathrm{BF}_{\bar{\Lambda} \rightarrow \bar{p} \pi^{+}}=0.639 \pm 0.005$ and $\mathrm{BF}_{\bar{\Sigma}^{-} \rightarrow \bar{p} \pi^{0}}=0.5157 \pm 0.0003$ are the branching fractions of the corresponding decays. While there are some existing data on the ratio $\bar{\Lambda} / \bar{p}$, there is not any data on the ratio $\bar{\Sigma}^{-} / \bar{p}$; and it is only estimated based on the symmetry argument that $\bar{\Sigma}^{-} / \bar{\Lambda}=0.33$ [388]. Thus, expression (60) can be rewritten as

$$
\Delta_{\Lambda}=(0.81 \pm 0.04) \times \bar{\Lambda} / \bar{p}
$$

Even existing data on the ratio $\bar{\Lambda} / \bar{p}$ has a high energy-dependent uncertainty ranging from $12 \%$ to $18 \%$ [386]. 


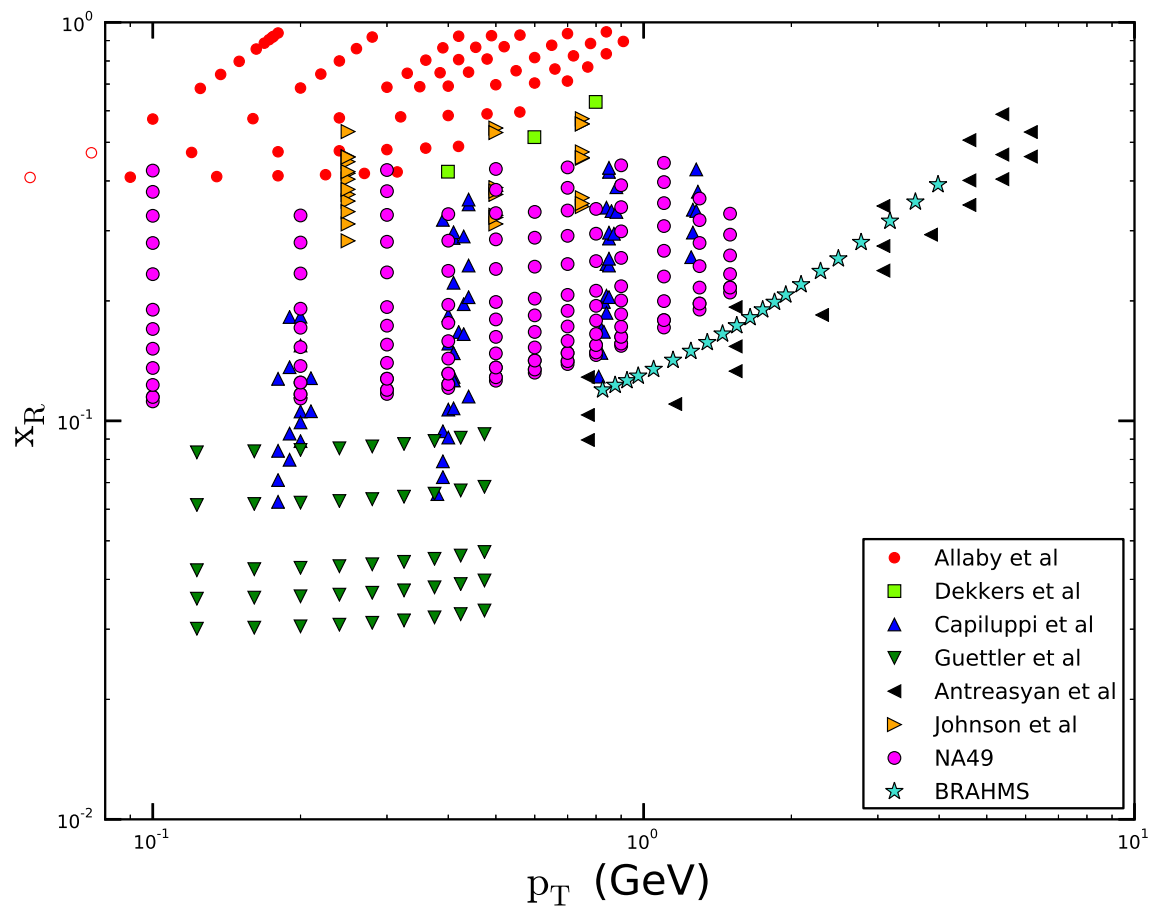

Fig. 42. The available data on $d^{3} \sigma_{p p \rightarrow \bar{p}} / d p^{3}$ in the $p_{T}-x_{R}$ space [382].

From all of the above, the need for new measurements, of the antiproton-production cross sections and hyperon-to-antiproton production ratios, becomes clear. The phase space that requires coverage in order to bridge the gap between antiproton data and the AMS-02 measurements has been outlined. Fig.43 shows it in terms of transverse momentum and the radial-scaling variable, where the areas within the contours need to be covered with uncertainty no more than $3 \%$, while the areas outside the contours require coverage with uncertainty no more than $30 \%$ [383]. 


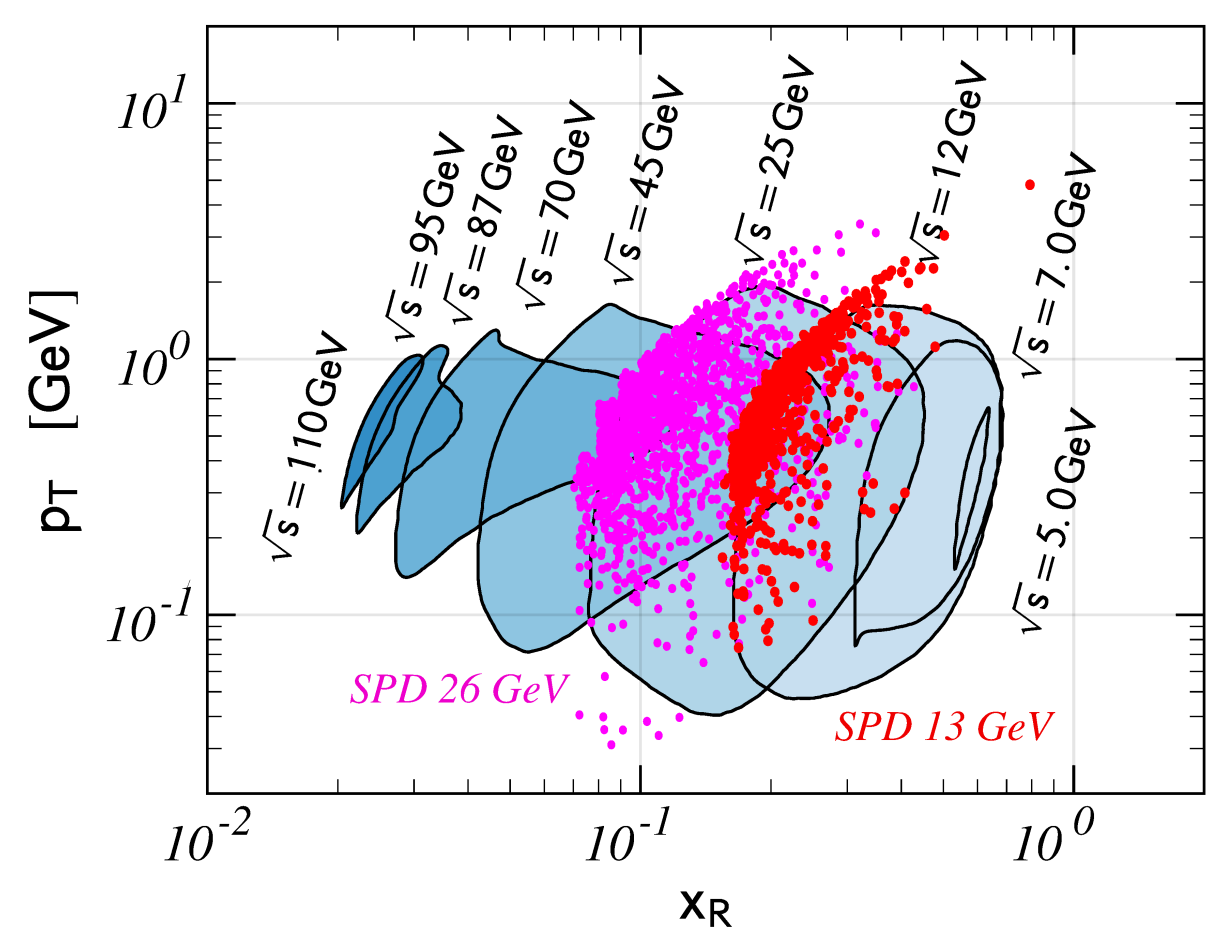

Fig. 43. The kinematic range accessible to the SPD [385] superimposed on the required measurement range to match the AMS-02 accuracy [383].

\subsection{NICA SPD Contribution}

The SPD detector is planned to operate at the NICA collider currently under construction at JINR [389]. Polarized proton and deuteron beams will be used. The $p p$ collisions are expected to reach $\sqrt{s}=27 \mathrm{GeV}$, and a luminosity of $5 \times 10^{30} \mathrm{~cm}^{-2} \mathrm{~s}^{-1}$ should be achieved [390]. Preliminary MC results using PYTHIA8 [391] show that the antiprotonproduction cross section in $p p$ collisions multiplied by the average antiproton-multiplicity in this energy range is on the level of a few millibarns [384], corresponding to a production rate $>10^{5} \mathrm{~s}^{-1}$. Thus, the measurements will not be hindered by statistical uncertainty. The $4 \pi$ angular acceptance of the SPD will also allow access to a wider kinematic range, in comparison to fixed-target experiments. Fig. 43 shows the kinematic range accessible to the SPD based on a preliminary MC study [385], superimposed on the required measurement range to match the AMS-02 accuracy.

The time-of-flight system of the SPD detector will enable particle identification. In addition, reconstruction of secondary-vertices will allow the study of secondary hyperon decays [385]. Thus, the SPD can also contribute to measurement of the $\bar{\Lambda} / \bar{p}$ ratio, where Fig. 44 shows the energy range accessible to the SPD. 


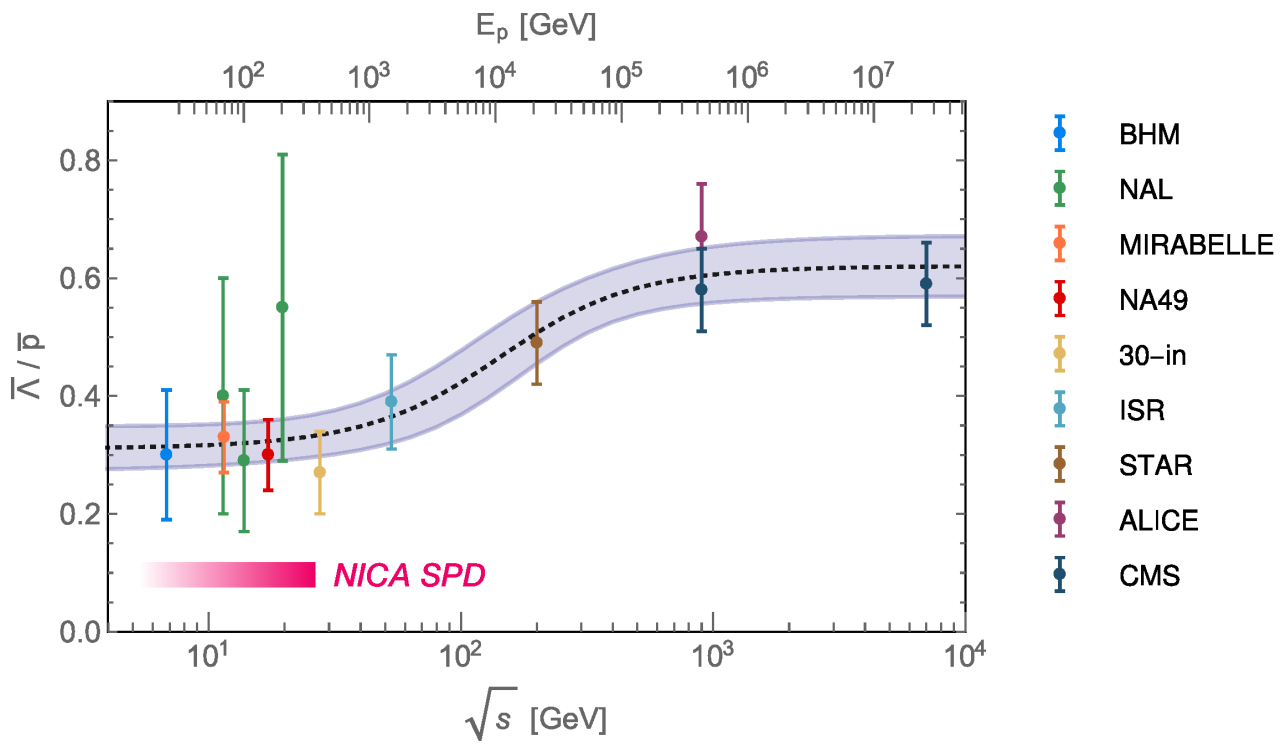

Fig. 44. $\bar{\Lambda} / \bar{p}$ ratio in proton-proton collisions as measured by several experiments [386]. The range of $\sqrt{s}$ accessible at the NICA SPD is indicated at the lower left part [385].

\subsection{Summary}

The Spin Physics Detector can make a sizable contribution to the search of physics beyond the Standard Model. The SPD can measure energy and angular distributions of antiprotons produced in proton-proton and proton-deuteron collisions both directly and from the decays of $\bar{\Lambda}$ and $\bar{\Sigma}^{-}$hyperons in the kinematic range starting from the threshold, for the astrophysical searches for dark matter. The collider mode and the geometry of the SPD detector provide a unique possibility to study the production of antiprotons at high transverse momenta which is unavailable for fixed-target experiments. The possibility for NICA to operate with beams of light nuclei, such as ${ }^{3} \mathrm{He}$ and ${ }^{4} \mathrm{He}$, could extend this program. 


\section{Tests of fundamental discrete symmetries at NICA facility: addendum to the spin physics programme ${ }^{1}$}

We present new ideas on tests of fundamental symmetries in polarization experiments at the NICA facility. Specifically, we explore the possibilities of high precision tests of the Standard Model by parity violation and searches of Beyond the Standard Model semistrong breaking of time reversal invariance in double polarized proton-deuteron scattering, taking advantage of high intensity beams of polarized protons and deuterons available at NICA. In both cases, we propose to use the new technique of polarized beam with precessing horizontal polarizations, and polarized deuterons are the favored choice. The external target in the extracted beam is optional for the parity violation experiment, which requires furnishing Nuclotron and/or new Booster with very modest new instrumentation. One should not overlook this potential for substantial broadening of horizons of spin physics at the NICA facility.

PACS: 13.40.Em, 11.30.Er, 29.20.Dh, 29.27.Hj

\section{Introduction}

The SPD project at NICA aims primarily at polarization experiments in the collider mode. In the broader context, the main virtue of NICA facility is high current beams of polarized protons and deuterons $[392,393]$. We report here new ideas developed in the framework of the Russian Fund for Basic Research Grant No. 18-02-40092 MEGA. Basically, we propose addendum to the previously discussed spin physics programme of the NICA facility. For decades to come, NICA will be a holder of a unique potential to conduct fixed target experimental tests of fundamental symmetries within or beyond the Standard Model (SM), and one should not overlook this outstanding opportunity.

Our knowledge about high energy parity violation (PV) in the pure non-leptonic sector of SM is as yet scarce. The most accurate result on PV asymmetry in $p p$ scattering at $45 \mathrm{MeV}, A_{P V}=-(1.5 \pm 0.22) \cdot 10^{-7}$, is based on the data collected for several years [394]. A result of PV experiment at ZGS wtih $5.1 \mathrm{GeV}$ protons interacting with the water target, $A_{P V}=-(26.5 \pm 6.0 \pm 3.6) \cdot 10^{-7}$, was also collected for several years [395]. Theorists failed to acomodate the latter, anomalously large, asymmetry in the SM (see review [396]). Only modest upper bounds were set at Fermilab in $p p$ and $p \bar{p}$ interactions at $200 \mathrm{GeV}: A_{P V}<10^{-5}$ [397].

Of our prime concern is a feasibility of precision measurements of PV asymmetry at the NICA facility [398-400]. Our key suggestion is using beams with polarization idly rotating in the ring plane. Such a polarization has for the first time been successfully applied in 1986 at the Budker INP, with participation of members of the present team, to a record precision comparison of magnetic anomalies of $e^{+}$and $e^{-}$[401]. We also mention the 2002 idea to accelerate oscillating polarization beams in Nuclotron [402]. Subsequently, the JEDI collaboration, including members of the present team, had extended this approach to studies of fine aspects of spin dynamics of polarized deuterons stored at the COSY accelerator [403-407]. The principal point is that time-stamped precessing polarization has matured, and can be viewed as useful as the static ones. Namely,

\footnotetext{
${ }^{1}$ This section is presented by I.A. Koop, A.I. Milstein, N.N. Nikolaev (E-mail: nikolaev@itp.ac.ru), A.S. Popov, S.G.Salnikov, P.Yu. Shatunov, Yu.M. Shatunov.
} 
oscillating spin asymmetries in cross section generated by the in-plane-precessing polarization, can readily be isolated by Fourier analysis. We find it imperative to dwell on the aspects of precessing polarization approach which are not that familiar to the NICA community.

In this report we mostly focus on the preferred external fixed target PV experiments at the new Booster or Nuclotron. The already published collaboration results [398-400] are mentioned only briefly. Execution of the PV experiment would require furnishing the Nuclotron or Booster ring with only very modest extra instrumentation: the radiofrequency spin flipper and internal target polarimeter to fix the time stamp of the spin phase. Extraction of the signal of PV violation from the difference of attenuations of extracted beams of opposite helicity will not require any sophisticated external detectors - the anticipated non-invasive measurement of the total charge of the bunch incident on the condensed matter target and of the transmitted bunch will be performed by a system of Rogowski coils.

Polarized deuterons are favored because the NICA energy range is free of the deuteron spin resonances. An outstanding record of experimentation with polarized deuterons at the Nuclotron is noteworthy $[408,409]$. Our starting point was the PV studies, but the project outgrew its original boundary: it was understood that the precessing polarization deuterons can give an access to tests of still another fundamental symmetry - the time reversal invariance $[410,411]$. Specifically, one can search for the semistrong T- and CP-violating, P-conserving and flavor-conserving interaction, suggested by Okun [412], Prentki \& Veltman [413], and Lee \& Wolfenstein [414]. An intriguing open issue is whether this manifestly beyond SM semistrong CP-violation can resolve the puzzle of the anomalously large baryon asymmetry of the Universe, where SM fails by many orders in magnitude [415]. On top of that, we mention also a possibility of spin crisis experiments with oscillating deuteron polarization at the electron-ion collider eIC [411].

\subsection{Precessing spin asymmetries in the total $p d$ cross section}

We illustrate the polarization effects on the example of total $p d$ cross section:

$$
\begin{aligned}
\sigma_{\text {tot }}= & \sigma_{0}+\sigma_{\mathrm{TT}}\left[\left(\mathbf{P}^{\mathrm{d}} \cdot \mathbf{P}^{\mathrm{p}}\right)-\left(\mathbf{P}^{\mathrm{d}} \cdot \mathbf{k}\right)\left(\mathbf{P}^{\mathrm{p}} \cdot \mathbf{k}\right)\right]+\sigma_{\mathrm{LL}}\left(\mathbf{P}^{\mathrm{d}} \cdot \mathbf{k}\right)\left(\mathbf{P}^{\mathrm{p}} \cdot \mathbf{k}\right)+\sigma_{\mathrm{T}} Q_{m n} k_{m} k_{n} \\
& +\sigma_{\mathrm{PV}}^{\mathrm{p}}\left(\mathbf{P}^{\mathrm{p}} \cdot \mathbf{k}\right)+\sigma_{\mathrm{PV}}^{\mathrm{d}}\left(\mathbf{P}^{\mathrm{d}} \cdot \mathbf{k}\right)+\sigma_{\mathrm{PV}}^{\mathrm{T}}\left(\mathbf{P}^{\mathrm{p}} \cdot \mathbf{k}\right) Q_{m n} k_{m} k_{n} \\
& +\sigma_{\mathrm{TVPV}}\left(\mathbf{k} \cdot\left[\mathbf{P}^{\mathrm{d}} \times \mathbf{P}^{\mathrm{p}}\right]\right)+\sigma_{\mathrm{TVPC}} k_{m} Q_{m n} \epsilon_{n l r} P_{l}^{\mathrm{p}} k_{r} .
\end{aligned}
$$

Here $\mathbf{P}^{\mathrm{d}}$ and $\mathbf{P}^{\mathrm{p}}$ are polarizations of the deuteron and proton, $Q_{m n}$ is the tensor polarization of the deuteron, and $\mathbf{k}$ is the collision axis (the $z$-axis). The $y$-axis is normal to the ring plane, $x$-axis is the radial one. In the tensor polarization dependent terms $Q_{m n} k_{m} k_{n}=Q_{z z}$, and $k_{m} Q_{m n} \epsilon_{n l r} P_{l}^{\mathrm{p}} k_{r}=Q_{x z} P_{y}^{\mathrm{p}}-Q_{y z} P_{x}^{\mathrm{p}}$ is odd under flip of the proton polarization. The cross sections $\sigma_{0}, \sigma_{\mathrm{TT}}, \sigma_{\mathrm{LL}}$, and $\sigma_{\mathrm{T}}$ correspond to the ordinary P-even and T-even interactions, $\sigma_{\mathrm{PV}}^{\mathrm{P}}, \sigma_{\mathrm{PV}}^{\mathrm{d}}$, and $\sigma_{\mathrm{PV}}^{\mathrm{T}}$ give $\mathrm{PV}$ signals , and $\sigma_{\mathrm{TVPV}}$ is the TVPV component, while $\sigma_{\text {TVPC }}$ is the null observable for the TVPC semistrong interaction $[20,30,31]$.The last three polarization effects have not been studied before. 
In our approach, the equilibrium vertical spin of the stored beam will be subjected to parametric resonance driven by the radiofrequency ( $\mathrm{RF}$ ) flipper (solenoid):

$$
\vec{P}(n)=P_{y}(0)\left[\cos (\epsilon n) \vec{e}_{y}+\sin (\epsilon n)\left[\cos \left(\theta_{s} n\right) \vec{e}_{x}-\sin \left(\theta_{s} n\right) \vec{e}_{z}\right] .\right.
$$

Here $n$ is the turn number, $\theta_{s}=2 \pi \nu_{s}$, where $\nu_{s}=G \gamma$ is the spin tune, $G$ is the magnetic anomaly of the particle, $\gamma$ is its relativistic $\gamma$-factor, $\epsilon=2 \pi \nu_{R}$ and $\nu_{R}$ is the spin resonance tune, related to the amplitude of spin rotation in a single pass through the spin flipper. In this equation, $\cos (\epsilon n)$ and $\sin (\epsilon n)$ have conspicuous interpretation as envelopes of the vertical and in-plane idly precessing polarizations, respectively. The flipper is turned off when $\epsilon n=\pi / 2$ is reached and then spin will keep idly precessing in the storage ring plane.

Oscillating $P_{z}=-P_{y}(0) \sin \left(\theta_{s} n\right)$ gives rise to the oscillating PV signal in total cross section, see Eq. 62. The internal target polarimetry of oscillating $P_{x}$ is used by JEDI collaboration to monitor the spin precession frequency [403,404] and simultaneously to provide the time stamp for $P_{z}$. In our proposal this time stamp will be used to trigger the single-turn dump of beam of the desired $P_{z}$ onto the external target. In JEDI experiments $[405,406]$ the spin coherence time of idly precessing deuterons has been maximized up to $1400 \mathrm{~s}$ by fine tuning the sextupole lenses, as was suggested already in 1987 by the present collaborations members (Koop and Shatunov [416]).

We mention briefly only major points about flipper driven evolution of the tensor polarization of deuterons starting with $Q_{y x}(0)=Q_{y z}(0)=Q_{x z}(0)=0$, and $Q_{x x}(0)=$ $Q_{z z}(0)=-\frac{1}{2} Q_{y y}(0)[411]:$

$$
\begin{aligned}
Q_{y y}(n) & =\frac{1}{2} Q_{y y}(0) \cdot\left[-1+3 \cos ^{2}(\epsilon n)\right], \\
Q_{x x}(n) & =\frac{1}{2} Q_{y y}(0) \cdot\left[-1+3 \sin ^{2}(\epsilon n) \cdot \cos ^{2}\left(\theta_{s} n\right)\right], \\
Q_{z z}(n) & =\frac{1}{2} Q_{y y}(0) \cdot\left[-1+3 \sin ^{2}(\epsilon n) \cdot \sin ^{2}\left(\theta_{s} n\right)\right], \\
Q_{y x}(n) & =\frac{3}{2} Q_{y y}(0) \cdot \sin (\epsilon n) \cdot \cos (\epsilon n) \cdot \cos \left(\theta_{s} n\right), \\
Q_{y z}(n) & =-\frac{3}{2} Q_{y y}(0) \cdot \sin (\epsilon n) \cdot \cos (\epsilon n) \cdot \sin \left(\theta_{s} n\right), \\
Q_{x z}(n) & =-\frac{3}{4} Q_{y y}(0) \cdot \sin ^{2}(\epsilon n) \cdot \sin \left(2 \theta_{s} n\right) .
\end{aligned}
$$

At the working point $\epsilon n=\pi / 2$ we have $Q_{y x}=Q_{y z}=0, Q_{y y}=$ const and $Q_{x x, z z} \propto 1 \pm$ $3 \cos 2 \theta_{s} n$. There is a conspicuous difference of Fourier harmonics of the vector and tensor polarizations, and the P-conserving effects of tensor polarizations are easily separated from the PV signal by Fourier analysis. On the other hand, the tensor asymmetry is well understood, and the signal of oscillating component of $Q_{z z}$ can serve as an important cross check of our technique. We emphasize that in search for the single-spin PV asymmetry one only needs unpolarized targets.

Of particular interest is the off-diagonal $Q_{x z}$. It enters the TVPC asymmetry which probes the semistrong CP-violating interaction $[20,30]$. The TVPC asymmetry $\propto Q_{x z}$ has the unique Fourier signature, is $P_{y}^{p}$-odd, and is free of systematic background [411]. 
However, it is the double-polarization observable, and search for semistrong CP-violation with stored polarized deuterons requires the internal polarized hydrogen target [410].

17.2. PV asymmetry: expectations from Standard Model

The theoretical results of the team were reported in three publications [399, 400,411]. The salient feature of the tree-level PV weak Hamiltonian is a strong suppression of the PV $p p$ amplitude, because numerically $\left|4 \sin ^{2} \theta_{W}-1\right| \ll 1$. However, the effective PV neutral current can be generated from charged current $n p$ interaction by radiative corrections from charge exchange strong interaction with encouraging magnitude, although the uncertainties are inevitably substantial [399]. The initial and final state strong interactions, aka absorption corrections, endow the initially real valued tree-level PV amplitude with the imaginary part, which can be evaluated in the eikonal approximation. The PV contribution to the total cross section is nearly exhausted by PV in elastic scattering, what entails a strong suppression of PV in inelastic scattering. The corollary is that in comparison to the total cross section, the PV asymmetry in elastic scattering will be enhanced by large factor of $\sigma_{t o t} / \delta \sigma_{e l} \sim 3$. The expectations from the SM for PV in proton-nucleon interactions at NICA are [399]: $\mathcal{A}_{t o t}^{p n} \sim 10^{-7}, \mathcal{A}_{t o t}^{p p} \sim 0.4 \cdot 10^{-7}$.

New features of $p d$ interactions are Glauber screening and quasielastic scattering, aka diffractive breakup of the deuteron $[417,418]$. The technicalities of calculations of P-odd asymmetries are found in the publication [400]. The enhancement of the P-odd asymmetry in elastic $p N$ scattering vs. total cross section will persist in both elastic and quasi-elastic $p d$ scattering. A substantial difference between the scattering of polarized deuterons on unpolarized protons and the scattering of polarized protons on unpolarized deuterons has its origin in the interference of the P-odd $p p$ and $p n$ amplitudes.

Remarkably, to the same accuracy as in the case of $p p$ scattering, the P-odd component of the total $p d$ cross section is exhausted by the sum of P-odd components of the total elastic and quasielastic cross sections. Now the strong suppression of the parity violation holds for truly inelastic $p d$ collisions with production of new particles (mesons).

We first report expectations for PV cross sections and asymmetries $\mathcal{A}=\sigma_{W} / \sigma_{s}$ in the scattering of polarized deuterons with $\lambda_{d}=1$ on unpolarized protons:

$$
\begin{aligned}
& \sigma_{s, \text { tot }}^{p d}=96 \mathrm{mb}, \quad \sigma_{W, \text { tot }}^{p d}=2.1 \mathrm{nb}, \quad \mathcal{A}_{t o t}^{p d}=2 * 10^{-8}, \\
& \sigma_{s, \text { el }}^{p d}=20 \mathrm{mb}, \quad \sigma_{W, e l}^{p d}=0.7 \mathrm{nb}, \quad \mathcal{A}_{e l}^{p d}=3.5 * 10^{-8}, \\
& \sigma_{s, q e l}^{p d}=22.4 \mathrm{mb}, \quad \sigma_{W, q e l}^{p d}=1.4 \mathrm{nb}, \quad \mathcal{A}_{q e l}^{p d}=6 * 10^{-8} .
\end{aligned}
$$

For the interaction of a polarized protons with $\lambda_{p}=1$ with unpolarized deuterons, we have

$$
\begin{array}{ll}
\sigma_{W, \text { tot }}^{p d}=-0.8 \mathrm{nb}, & \mathcal{A}_{t o t}^{p d}=-0.9 * 10^{-8}, \\
\sigma_{W, \text { el }}^{p d}=-0.6 \mathrm{nb}, & \mathcal{A}_{e l}^{p d}=-3 * 10^{-8} \\
\sigma_{W, q e l}^{p d}=-0.2 \mathrm{nb}, & \mathcal{A}_{q e l}^{p d}=-10^{-8}
\end{array}
$$




\subsection{The experimental strategies}

Generic considerations of the external target option. The analysis of the optimal strategy for the PV experiment is in the formative stage. To illustrate the challenges one faces, we focus here on the option of extracted polarized deuteron beams interacting with the external target, similar to that used in the ZGS experiment with accelerated protons [395]. A source of polarized deuterons with unique parameters [408] has been commissioned at the Joint Institute for Nuclear Research decades ago, and successful sessions of their acceleration in the Nuclotron [409] have been carried out. According to [393], the Nuclotron is able to accelerate in one cycle up to $1.6 \cdot 10^{11}$ polarized protons and deuterons.

The principal target is a reach to PV asymmetries at the level $\sim 10^{-7}$ or better. On generic grounds that requires about $10^{15}$ events. The physical observable will be a difference of attenuations of the positive and negative helicity beams in thick dense target. To maximize the statistics, one needs large number of cycles. The typical cycle will consist of (1) injection of vertically polarized particles, (2) acceleration to the required energy, (3) rotation of polarization from the vertical to horizontal one by RF flipper, (4) the polarimetry of the in-plane precessing spin and the determination of the spin-tune and the spin phase, (5) single-turn extraction of the beam of desired helicity onto the target, (6) comparison of beam currents upstream and downstream of the target.

We skip a discussion of the routine stages (1) and (2).

The spin coherence time. The stages (3) and (4) require more attention. The vertical polarization is preserved by the vertical guiding field in the ring. The in-plane idly precessing spins decohere with time. Therefore, the stages (3) and (4) together must be shorter than the spin coherence time. Ever since the experiment [401], the RF flippers are being routinely used in the spin experiments. More detailed discussion of the proposed fast flipper will be presented below, for the purposes of the present discussion it suffices to know that the vertical spin can be rotated to the horizontal one faster than in $1 \mathrm{~s}$. As the reference point we cite the JEDI result, that with beams of $10^{9}$ deuterons of momentum $0.97 \mathrm{GeV} / \mathrm{c}$ in COSY, the spin precession phase can be measured to the accuracy of $\sim 0.2$ in $2 \mathrm{~s}[404,407]$. Steady operation with spin coherence time exceeding $1000 \mathrm{~s}$ has been achieved [405,406]. The educated guess is that, at the same energy of deuterons, the spin coherence time will be sufficient for less that $3 \mathrm{~s}$ of idle precession in Nuclotron or the new Booster rings even without cooling the beam and a single cycle can be as short as $\sim 5 \mathrm{sec}$. The radial polarization cycles bring the effective cycle length to $\sim 10 \mathrm{~s}$. Making allowance for the contingency factor of 2 , we end up with $\sim 130000$ effective cycles per month. By the rule of thumb, in one month data taking with thick target of one absorption length, the total number of interactions can reach $\sim 10^{16}$. A parasitic data taking, when Nuclotron and/or Booster are idling during operation of NICA in the collider mode, makes possible a further gain in the statistics.

Polarization of the ejected beam. One needs the single-turn extraction of the stored bunch. At the discussed energy, the spin tune of deuterons $\nu_{s}=G \gamma=-0.160977$. After 50 particle revolutions in the ring, the spin will make 8.048 in-plane rotations, after 99 revolutions the spin will make 15.966 rotations, and 23.986 in-pane rotations after 149 revolutions etc. This shows that with time stamp it will take not much longer than few 
hundred turns of the beam, i.e.,, few decimal fractions of a millisecond, to decide when to extract the beam polarized in any desired orientation. A good option is a sequence of two cycles with alternating $P_{z}$ to measure the PV asymmetry, and two more cycles to crosscheck the equality of attenuations of beams with alternating radial $P_{x}$. Tensor asymmetry of the total cross section is large and PV cycles can easily be interspersed with the control sequence of cycles to extract the second harmonics signal from the precessing tensor polarization.

Polarimetry issues. Internal cylindrical scintillation polarimeter made of four, topbottom and right-left sectors, will provide time resolution to dynamically measure the oscillating transverse polarization of the beam from the oscillating up-down asymmetry $[403,404]$. The periphery of the beam can be brought to collisions with the carbon target either by stochastic heating of the beam or generating the bump by beam steerers. The polarimetry will consume only a small fraction of the beam before it is ejected into the target channel. A cycle-to-cycle stability of orbits will be controlled by beam position monitors along the ring circumference, the magnetic field will be controlled by NMR sensors in a special dipole magnet powered serially with the ring dipoles. Specific to the approach is a high precision cycle-to-cycle comparison of spin tunes, which amounts to a comparison of energies. The supplementary polarimetry of the beam after the target will provide important cross check of orientation of the polarization vector of the beam incident on the target.

Flipper implementation issues. For deuterons with momentum $p=0.97 \mathrm{GeV} / c$ (kinematic parameters $\gamma=1.125, \beta=0.46$ ), it is rational to apply the longitudinal magnetic field oscillating at a relatively low frequency $f=f_{c} \cdot \gamma|G|=88.3 \mathrm{kHz}$. The ceramic vacuum chamber must have conductive longitudinal stripes on the inner surface so that the beam image currents can freely propagate along these metalized tracks. The outer side of the ceramic chamber will serve as a skeleton for winding the solenoid turns. The approximate technical parameters of the flipper [398] are shown in Table 7. The necessary power to the RF generator, $5 \mathrm{~kW}$, can be provided, for instance, by the generator triode GI-50, capable of delivering up to $40 \mathrm{~kW}$ of power in a continuous mode, with the help of modern semiconductor amplifiers. We leave these issues for a future technical study.

The accuracy issues in the external target mode. With $N_{1}$ particles impinging on the target and $N_{2}$ particles left behind the target, the total beam loss cross section $\sigma_{\text {tot }}$ per target nucleus is derived from the exponential attenuation law, $N_{2}=N_{1} \cdot \exp \left(-\sigma_{\text {tot }} \rho\right)$, where $\rho$ is target density: $\sigma_{\text {tot }}=\rho^{-1} \ln \left(N_{1} / N_{2}\right), \quad \delta \sigma_{\text {tot }}=\rho^{-1}\left(\delta N_{1} / N_{1}-\delta N_{2} / N_{2}\right)$. We estimate the dispersion of the measured number of particles $N$ following the $\sqrt{N}$ law, so that $\left\langle\delta N_{1}^{2}\right\rangle=N_{1}$. For the transmitted beam, allowance for the dispersion of the transmission coefficient $p$ gives the corrected formula $\left\langle\delta N_{2}^{2}\right\rangle=N_{2}+p(1-p) N_{1}$. The best rms accuracy of measuring loss cross-section is achieved at $p=e^{-2}$ :

$$
\frac{\delta\left(\sigma_{\text {tot }}\right)}{\sigma_{\text {tot }}}=\sqrt{\frac{2}{p \ln ^{2} p}} \cdot \frac{1}{\sqrt{N_{1}}} \Rightarrow \frac{e}{\sqrt{2}} \cdot \frac{1}{\sqrt{N_{1}}}=\frac{1.92}{\sqrt{N_{1}}} .
$$

Above we argued that $\sim 1.6 \cdot 10^{5}$ cycles per month are feasible. Consequently, in order to achieve the statistical accuracy of $10^{-7}$ in measuring the loss cross-section in the onemonth run, it is necessary to ensure the accuracy of the asymmetry measurement in the 
Table 7. The main flipper parameters for the deuteron momentum $0.97 \mathrm{GeV} / c$ and the amplitude of its circular harmonic $w=2.5 \cdot 10^{-5}$ (field integral $B l=1.2 \cdot 10^{-3} \mathrm{~T} \cdot \mathrm{m}$ )

$\begin{array}{lll}\text { Solenoid length } & 1.0 & \mathrm{~m} \\ \text { Magnetic field amplitude } & 0.0012 & \mathrm{~T} \\ \text { Spiral winding diameter } & 150 & \mathrm{~mm} \\ \text { Aperture of the ceramic vacuum chamber } & 120 & \mathrm{~mm} \\ \text { Case diameter } & 400 & \mathrm{~mm} \\ \text { Solenoid turns } & 80 & \\ \text { Winding inductance } & 150 & \mu \mathrm{H} \\ \text { Characteristic impedance of the circuit } & 75 & \mathrm{Ohm} \\ \text { Active loss resistance } & 0.2 & \mathrm{Ohm} \\ \text { Quality factor of the oscillating circuit } & 375 & \\ \text { Winding current } & 150 & \mathrm{~A} \\ \text { Inductive voltage } & 11 & \mathrm{kV} \\ \text { Active loss power } & 4.5 & \mathrm{~kW}\end{array}$

single cycle at the level of $A_{1} \approx 4 \cdot 10^{-5}$. The number of particles in the bunch impinging on the target must be no less than $\approx 2.3 \cdot 10^{9}$. This leaves a certain room for the further improvement of sensitivity to PV asymmetry increasing the number of particles in the bunch. Furthermore, in the parasitic mode the data taking can be stretched beyond one month.

One can view two options to measure the number of particles in the beam. The first one is to resort to ionization chambers or secondary emission sensors with multiplication of secondary particles. With $n$ secondary particles produced per one primary particle in the final state, total number of secondary particles will be $N \cdot n$, what entails the relative rms fluctuation in the determination of the number of particles in the beam

$$
\frac{\delta N}{N}=\frac{\delta(N n)}{N n}=\sqrt{\frac{1}{N n}}
$$

An alternative option is a non-destructive measurement of the total charge of the bunch before and after interaction in the target - a comparison of charges before and after amounts to desired comparison of particle numbers $N_{1,2}$. Such an approach will take advantage of a bunched beam required for the time stamp of the precessing polarization. Namely, the Rogowski coils with high permeability amorphous iron core are known to be good transformers of the current from the primary circuit, i.e., the beam current, to the secondary circuit with a very high degree of identity. This is largely due to the very large ratio of the magnetizing inductance of the core to the leakage inductance.

The primary signal from the Rogowski coil is a voltage proportional to the time derivative of the beam current: $U=L \dot{I}$. This signal is applied to the infinitely large resistance of the amplifier buffer stage. Next, it is subjected to analog integrations on operational amplifiers (OA), composed of an $\mathrm{RC}$ chain. The first integration will give at the output a signal $U_{1}(t)=L \cdot I(t) / R_{1} C_{1}$. After the second integration, we get at the output an almost constant voltage $U_{2}$ and the accumulated charge $q_{2}$ on the capacitor 
$C_{2}$ will equal

$$
U_{2}=\frac{q \cdot L}{R_{1} R_{2} C_{1} C_{2}}, \quad q_{2}=\frac{q \cdot L}{R_{1} R_{2} C_{1}} .
$$

With a large ratio $L /\left(R_{1} R_{2} C_{1}\right)$, one can get a significant gain in the accumulated charge on the capacitor of the second integrator. Note that the values of the time constants of the RC chains do not in any way affect the linearity of signal conversion by the integrator on the OA, in contrast to its passive analogue, where the signal is integrated imperfectly, with damping determined by the time constant $\tau=R C$. Leaving aside the question of the magnitude of the noise in the signal processing circuit for the current coils, we can state that it is promising to use the above approach to measure the transmission coefficient of a beam through a dense target in the transport channel from the Nuclotron or the new Booster.

One can further increase the overall statistical accuracy of measuring the beam transmission coefficient installing 3-5 identical devices both in front of, and after the target. Besides better statistics, that will allow for the mutual control of the received data from all sensors. In principle, the above considerations of performance of the Rogowski coils as beam current transformers can be studied in the test stand experiment simulating the particle bunches by the current pulses.

\subsection{Summary and outlook}

High intensity beams of polarized deuterons available at NICA facility make feasible a high precision PV tests of the Standard Model. We consider the external fixed dense target experiments at either Nuclotron or new Booster the most promising ones. At the core of our proposal is a new technique of polarization precessing in the ring plane, which enables one to eject onto the target beams of any desired spin orientation. We anticipate a non-invasive measurement of the total charge of the bunch incident on the condensed matter target and of the transmitted jne as well by a system of Rogowski coils, so that the PV experiment will not require any sophisticated external detectors. The only two new devices, the RF spin flipper and internal target polarimeter, can be made sufficiently compact to fit into the Nuclotron and/or the new Booster ring. The Booster may be preferred for the less crowded ring lattice. A possibility of conducting the PV asymmetry experiment in the parasitic mode needs more scrutiny. There are still open questions, but by statistics considerations, the PV asymmetries smaller than $10^{-7}$ are within the reach of the proposed scheme.

For the lack of space, we omitted a number of items, including the spin resonance issues in operation with polarized protons, possible PV experiment with the internal dense target, selection of elastic events at high energies etc. We sketched only briefly a search for the semistrong $\mathrm{CP}$ violation which requires the internal polarized proton $\mathrm{ABS}$ target. The theoretical analysis of PV in polarized deuteron-nucleus interactions is in progress.

At electron-ion colliders, one can not produce longitudinal polarization of deuterons resorting to Siberian snakes, because of the impractically large required field integrals. The ideas of operation at the integer spin tune, developed at JINR [419-421], have been further extended at BNL $[422,423]$. In view of simplicity of the approach, a fresh 
look at the possibility of oscillating in-plane polarization of ultra-relativistic deuterons is worthwhile. A solution has to be found to increase the horizontal spin coherence time of $\sim 1400 \mathrm{~s}$, achieved so far at COSY [405], by more than one order of magnitude to match the expected storage time of $\sim 10 \mathrm{~h}$ at eIC [424]. 


\section{Acknowledgments}

V. Baskov, O. Dalkarov, A. L'vov and V. Polyanskiy acknowledge a support by the Russian Fund for Basic Research, Grant No. 18-02-40061. V.A. Ladygin acknowledges the support by the Russian Fund for Basic Research, Grant No.19-02-00079a. A. Larionov acknowledges the support by the Frankfurt Center for Scientific Computing and financial support by the German Federal Ministry of Education and Research (BMBF), Grant No. 05P18RGFCA. I.A. Koop, A.I. Milstein, N.N. Nikolaev, A.S. Popov, S.G. Salnikov, P.Yu. Shatunov,Yu.M. Shatunov acknowledge a support by the Russian Fund for Basic Research, Grant No. 18-02-40092 MEGA. 


\section{REFERENCES}

1. Epelbaum E. et al. [LENPIC Collaboration] Few- and many-nucleon systems with semilocal coordinate-space regularized chiral two- and three-body forces // Phys. Rev. C. - 2019. - V. 99, no. 2. - P. 024313. - arXiv:1807.02848.

2. Epelbaum E., Krebs H., Reinert P. High-precision nuclear forces from chiral EFT: State-of-the-art, challenges and outlook // Front. in Phys. - 2020. - V. 8. - P. 98. arXiv:1911.11875.

3. Carlson J., Gandolfi S., Pederiva F., Pieper S.C., Schiavilla R., Schmidt K., Wiringa R. Quantum Monte Carlo methods for nuclear physics // Rev. Mod. Phys. 2015. - V. 87. - P. 1067. - arXiv:1412.3081 [nucl-th].

4. Abazov V.M., others. Conceptual design of the Spin Physics Detector. - 2021.1. - arXiv:2102.00442.

5. Mueller A. in Proceedings of 17th rencontre de Moriond, Moriond, 1982 Van (Editions Frontieres, Gif-sur-Yvette, France, 1982) . - P. 13-20.

6. Brodsky S. in Proceedings of the 13th Int. Symposium on Multiparticle Dynamics, W. Kittel, W. Metzger and A. Stergiou (eds.) Singapore 1982, p.963. - 1982.

7. Clement H. On the History of Dibaryons and their Final Observation // Prog. Part. Nucl. Phys. - 2017. - V. 93. - P. 195. - arXiv:1610.05591.

8. Brodsky S.J., de Teramond G. Spin Correlations, QCD Color Transparency and Heavy Quark Thresholds in Proton Proton Scattering // Phys. Rev. Lett. - 1988. V. 60.- P. 1924.

9. Court G., others. Energy Dependence of Spin Effects in $p$ (Polarized) $p$ (Polarized) $\rightarrow p p / /$ Phys. Rev. Lett. - 1986. - V. 57. - P. 507.

10. Aaij R. et al. [LHCb Collaboration] Observation of $J / \psi p$ Resonances Consistent with Pentaquark States in $\Lambda_{b}^{0} \rightarrow J / \psi K^{-} p$ Decays // Phys. Rev. Lett. - 2015. - V. 115. - P. 072001. - arXiv:1507.03414.

11. Arndt R., Briscoe W., Strakovsky I., Workman R. Updated analysis of NN elastic scattering to $3-\mathrm{GeV} / /$ Phys. Rev. C. - 2007. - V. 76. - P. 025209.arXiv:0706.2195 [nucl-th].

12. Sawamoto M., Wakaizumi S. Analysis of elastic p p scattering at $6-\mathrm{GeV} / \mathrm{c}$ with spin orbit and spin spin coupling eikonals // Prog. Theor. Phys. - 1979. - V. 62. P. 563-565.

13. Selyugin O., Teryaev O. Generalized Parton Distributions and Description of Electromagnetic and Graviton form factors of nucleon // Phys. Rev. D. - 2009.V. 79. - P. 033003. - arXiv:0901.1786 [hep-ph].

14. Selyugin O. High Energy Hadron Spin Flip Amplitude // Phys. Part. Nucl. Lett. 2016. - V. 13, no. 3. - P. 303-309. - arXiv:1512.05130. 
15. Ford W.P., Van Orden J. Regge model for nucleon-nucleon spin-dependent amplitudes // Phys. Rev. C. - 2013. - V. 87, no. 1. - P. 014004. - arXiv:1210.2648 [nuclth].

16. Sibirtsev A., Haidenbauer J., Hammer H.W., Krewald S., Meißner U.G. Protonproton scattering above $3 \mathrm{GeV} / \mathrm{c} / /$ Eur. Phys. J. A. - 2010. - V. 45. - P. 357-372. arXiv:0911.4637 [hep-ph].

17. Bystricky J., Lehar F., Winternitz P. ON TESTS OF TIME REVERSAL INVARIANCE IN NUCLEON NUCLEON SCATTERING. - 1982. - 10.

18. Bystricky J., Lehar F., Winternitz P. Formalism of Nucleon-Nucleon Elastic Scattering Experiments // J. Phys. (France). - 1978. - V. 39. - P. 1.

19. Platonova M., Kukulin V. Refined Glauber model versus Faddeev calculations and experimental data for $p d$ spin observables // Phys. Rev. C. - 2010. - V. 81. P. 014004. - [Erratum: Phys.Rev.C 94, 069902 (2016)] arXiv:1612.08694.

20. Temerbayev A., Uzikov Y. Spin observables in proton-deuteron scattering and Tinvariance test // Phys. Atom. Nucl. - 2015. - V. 78, no. 1. - P. 35-42.

21. Mchedlishvili D., others. Deuteron analysing powers in deuteron-proton elastic scattering at 1.2 and $2.27 \mathrm{GeV} / /$ Nucl. Phys. A. - 2018. - V. 977. - P. 14-22.arXiv:1805.05778.

22. Platonova M.N., Kukulin V.I. Theoretical study of spin observables in $p d$ elastic scattering at energies $T_{p}=800-1000 \mathrm{MeV} / /$ Eur. Phys. J. A. $-2020 .-$ V. 56, no. 5. - P. 132. - arXiv:1910.05722.

23. Uzikov Y., Haidenbauer J., Bazarova A., Temerbayev A. Spin observables of protondeuteron elastic scattering at SPD NICA energies within the Glauber model and pN amplitudes Talk at NUCLEUS-2020, (11-17 October, 2020; S-Petersburg, Russia) . Submitted to PEPAN. - 2020. - 11. - arXiv:2011.04304.

24. Parry J., Booth N., Conforto G., Esterling R., Scheid J., Sherden D., Yokosawa $A$. Measurements of the polarization in proton proton elastic scattering from 2.50 to $5.15 \mathrm{gev} / \mathrm{c} / /$ Phys. Rev. D. - 1973. - V. 8. - P. 45-63.

25. Abshire G., Ankenbrandt C., Crittenden R., Heinz R., Hinotani K., Neal H., Rust D. Polarization structure in p-p elastic scattering, // Phys. Rev. Lett. - 1974. V. 32. - P. 1261-1264.

26. Corcoran M., others. Proton Polarization in p p Elastic and Inclusive Processes at Beam Momenta From 20-GeV/c to 400-GeV/c // Phys. Rev. D. - 1980. - V. 22. P. 2624. - [Erratum: Phys.Rev.D 24, 3010 (1981)].

27. Gaidot $A$. et al. [SACLAY-SERPUKHOV-DUBNA-MOSCOW Collaboration] Polarization Measurements in pi $+\mathrm{p}, \mathrm{K}+\mathrm{p}$ and p p Elastic Scattering at $45-\mathrm{GeV} / \mathrm{c}$ and Comparison with Regge Phenomenology // Phys. Lett. B. - 1976. - V. 61. P. 103-106. 
28. Ohlsen G.G. Polarization transfer and spin correlation experiments in nuclear physics // Rept. Prog. Phys. - 1972. - V. 35. - P. 717-801.

29. Uzikov Y., Wilkin C. The analysing powers in proton-deuteron elastic scattering // Phys. Lett. B. - 2019. - V. 793. - P. 224-226. — arXiv:1902.03596.

30. Uzikov Y.N., Temerbayev A. Null-test signal for $T$-invariance violation in $p d$ scattering // Phys. Rev. C. - 2015. - V. 92, no. 1. - P. 014002. - arXiv:1506.08303.

31. Uzikov Y.N., Haidenbauer J. Polarized proton-deuteron scattering as a test of time-reversal invariance // Phys. Rev. C. - 2016. - V. 94, no. 3. - P. 035501.arXiv:1607.04409.

32. Selyugin $O$. Gravitation form-factors and spin asymmetries in hadron elastic scattering. - 2010. - 8. - arXiv:1008.1323 [hep-ph].

33. Goggi G., Cavalli-Sforza M., Conta C., Fraternali M., Mantovani G., Pastore F., Alberi $G$. Inelastic Intermediate States in Proton - Deuteron and Deuteron-deuteron Elastic Collisions at the $\{$ ISR $\} / /$ Nucl. Phys. B. - 1979. - V. 149. - P. 381-412.

34. Allaby J., others. Structure in the angular distribution of high energy proton-proton scattering // Phys. Lett. B. - 1968. - V. 28. - P. 67-71.

35. Akerlof C., Hieber R., Krisch A., Edwards K., Ratner L., Ruddick K. Elastic Proton-Proton Scattering at 90-degrees and Structure within the Proton // Phys. Rev. - 1967. - V. 159. - P. 1138-1149.

36. Perl M.L., Cox J., Longo M.J., Kreisler M. NEUTRON - PROTON ELASTIC SCATTERING FROM 2-GeV/c TO 7-GeV/c // Phys. Rev. D. - 1970. - V. 1. P. 1857.

37. Stone J., Chanowski J., Gustafson H., Longo M., Gray S. Large Angle NeutronProton Elastic Scattering from 5-GeV/c to 12-GeV/c // Nucl. Phys. B. - 1978. V. 143. - P. 1-39.

38. Crabb D., others. Spin Dependence of High p-Transverse**2 Elastic p p Scattering // Phys. Rev. Lett. - 1978. - V. 41. - P. 1257.

39. Crosbie E., others. Energy Dependence of Spin Spin Effects in p p Elastic Scattering at 90-Degrees Center-Of-Mass // Phys. Rev. D. - 1981. - V. 23. - P. 600.

40. Sargsian M.M. Nucleon - Nucleon Interactions at Short Distances. - 2014. - 3. arXiv:1403.0678 [nucl-th].

41. Brodsky S.J., Carlson C.E., Lipkin H.J. Spin Effects in Large Transverse Momentum Exclusive Scattering Processes // Phys. Rev. D. - 1979. - V. 20. - P. 2278 2289.

42. Farrar G.R., Gottlieb S.A., Sivers D.W., Thomas G.H. Constituent Description of n n Elastic Scattering Observables at Large Angles // Phys. Rev. D. - 1979. V. 20. - P. 202 
43. Mardor I., others. Nuclear transparency in large momentum transfer quasielastic scattering // Phys. Rev. Lett. - 1998. - V. 81. - P. 5085-5088.

44. Aclander J., others. Nuclear transparency in 90 degree c.m. quasielastic A(p,2p) reactions // Phys. Rev. C. - 2004. - V. 70. - P. 015208. - arXiv:nucl-ex/0405025.

45. Bhetuwal D. et al. [Hall C Collaboration] Ruling out color transparency in quasielastic ${ }^{12} \mathrm{C}(\mathrm{e}, \mathrm{e} ' \mathrm{p})$ up to $Q^{2}$ of $14.2(\mathrm{GeV} / \mathrm{c})^{2}$. - 2020. - 11. - arXiv:2011.00703.

46. Ralston J.P., Pire B. Oscillatory Scale Breaking and the Chromo - Coulomb Phase Shift // Phys. Rev. Lett. - 1982. - V. 49. - P. 1605.

47. Brodsky S.J. Novel QCD physics at NICA // Eur. Phys. J. A. - 2016. - V. 52, no. 8. - P. 220.

48. Rekalo M.P., Tomasi-Gustafsson E. Threshold J / psi production in nucleonnucleon collisions // New J. Phys. - 2002. - V. 4. - P. 68. — arXiv:nucl-th/0204066.

49. Amaldi U., Schubert K.R. Impact Parameter Interpretation of Proton Proton Scattering from a Critical Review of All ISR Data // Nucl. Phys. B. - 1980. - V. 166. P. 301-320.

50. Anselm A., Gribov V. Zero pion mass limit in interactions at very high-energies // Phys. Lett. B. - 1972. - V. 40. - P. 487-490.

51. Khoze V., Martin A., Ryskin M. t dependence of the slope of the high energy elastic pp cross section // J. Phys. G. - 2015. - V. 42, no. 2. - P. 025003.arXiv:1410.0508 [hep-ph].

52. Jenkovszky L., Szanyi I., Tan C.I. Shape of Proton and the Pion Cloud // Eur. Phys. J. A. - 2018. - V. 54, no. 7. - P. 116. - arXiv:1710.10594.

53. Antipov Yu.M., Bessubov V.A., Denisenko A.A., Denisov S.P., Gorin Yu.P., Lebedev A.A., Mikhailov Yu.V., Petrukhin A.I., Polovnikov S.A., Roinishvili V.N., Soldatov M.M., Stoyanova D.A., Yotch F.A. Measurements of $\pi^{+} \mathrm{p}, K^{+} \mathrm{p}$ and $p p$ Elastic Scattering // Czech. J. Phys.B. - 1976. - V. 26. - P. 382-387. - Preprint IHEP PEPh 76-85, Serpukhov 1976, 44 p.

54. Denisov S.P., Kozelov A.V., Petrov V.A. On the possibility for precision measurements of differential cross sections for elastic proton-proton scattering at the Protvino accelerator // Phys. Atom. Nucl. - 2016. - V. 79, no. 2. - P. 199-206.[Yad. Fiz. 79, no.2, 121 (2016)].

55. Schiz A., Fajardo L.A., Majka R., Marx J.N., Nemethy P., Rosselet L., Sandweiss J., Slaughter A.J., Ankenbrandt C., Atac M., Brown R., Ecklund S., Gollon P.J., Lach J., MacLachlan J., Roberts A., Shen G. A High Statistics Study of $\pi^{+} p, \pi^{-} p$, and $p p$ Elastic Scattering at $200 \mathrm{GeV} / \mathrm{c} / /$ Phys. Rev.D. - 1981. - V. 24. - P. 26. 
56. Belousov V.I., Ezhela V.V., Tkachenko N.P. Combined Description of the Total and Differential Cross Sections and the $\rho$-Parameter for $p(\bar{p}) p$ Scattering over the Energy Region of $\sqrt{s} \geqslant 7 \mathrm{GeV}$ and for all Values of $t<0 / /$ Phys. Atom. Nucl. 2020. - V. 83, no. 5. - P. 720-730. - [Yad. Fiz.83,no.5,433(2020)].

57. Dremin I.M. Elastic scattering of hadrons // Phys. Usp. - 2013. - V. 56. - P. 328. - [Usp. Fiz. Nauk183,3(2013)] arXiv:1206.5474 [hep-ph].

58. Selyugin O.V. Nucleon structure and the high energy interactions // Phys. Rev.D. 2015. - V. 91, no. 11. - P. 113003. - [Erratum: Phys. Rev.D92,no.9,099901(2015)] arXiv:1505.02426.

59. Prochazka J., Kundrat $V$. Eikonal model analysis of elastic proton-proton collisions at $52.8 \mathrm{GeV}$ and $8 \mathrm{TeV} / /$ Eur. Phys. J.C. - 2020. - V. 80, no. 8. - P. 779.arXiv:1606.09479.

60. Broniowski W., Jenkovszky L., Ruiz Arriola E., Szanyi I. Hollowness in $p p$ and $p \bar{p}$ scattering in a Regge model // Phys. Rev.D. - 2018. - V. 98, no. 7. - P. 074012.arXiv:1806.04756.

61. Sharov $V$. Selection of the elastic scattering events in interactions of the NICA colliding proton (deuteron) beams // EPJ Web Conf. - 2017. - V. 138. - P. 11002.

62. Bogolyubov N.N., Shirkov D.V. Quantum Fields. - Benjamin-Cummings Pub. Co, 1982.

63. Goodhew H., Jazayeri S., Pajer E. The Cosmological Optical Theorem. - 2020. 9. - arXiv:2009.02898.

64. Martin A. Scattering Theory: Unitarity, Analyticity and Crossing. - 1969. - V. 3.

65. Roy S. High energy theorems for strong interactions and their comparison with experimental data // Phys. Rept. - 1972. - V. 5. - P. 125-196.

66. Dremin I. Critical behavior of cross sections at LHC // Int. J. Mod. Phys. A. 2016. - V. 31, no. 19. - P. 1650107. - arXiv:1605.08216.

67. Dremin I. Unexpected properties of interaction of high-energy protons // Usp. Fiz. Nauk. - 2017. - V. 187, no. 4. - P. 353-366.

68. Ji X.D. Deeply virtual Compton scattering // Phys. Rev. D. - 1997. - V. 55.P. 7114-7125. - arXiv:hep-ph/9609381.

69. Anselmino M., Forte S. Small angle polarization in high-energy p p scattering through nonperturbative chiral symmetry breaking // Phys. Rev. Lett. - 1993. V. 71. - P. 223-226. - arXiv:hep-ph/9211221.

70. Dorokhov A., Kochelev N., Zubov Y. Four quark states and nucleon - anti-nucleon annihilation within the quark model with QCD vacuum induced interaction // Z. Phys. C. - 1995. - V. 65. - P. 667-680. - arXiv:hep-ph/9412378. 
71. Edneral V., Troshin S., Tyurin N. On Spin Effects in Elastic Scattering at Large Momentum Transfers // JETP Lett. - 1979. - V. 30. - P. 330.

72. Selyugin O.V. Coulomb hadron phase factor and spin phenomena in a wide region of transfer momenta // Phys. Rev. D. - 1999. - V. 60. - P. 074028.

73. Selyugin O. GPDs of the nucleons and elastic scattering at high energies // Eur. Phys. J. C. - 2012. - V. 72. - P. 2073. - arXiv:1201.4458 [hep-ph].

74. Buttimore N.H., Gotsman E., Leader E. SPIN DEPENDENT PHENOMENA INDUCED BY ELECTROMAGNETIC HADRONIC INTERFERENCE AT HIGHENERGIES // Phys. Rev. D. - 1978. - V. 18. - P. 694-716. - [Erratum: Phys.Rev.D 35, 407 (1987)]

75. Selyugin O.V. Phase of the Coulomb amplitude in the second Born approximation. // Mod. Phys. Lett. A. - 1996. - V. 11. - P. 2317-2323.

76. Selyugin O.V. Hadron spin flip amplitude and slope of differential cross-sections // Mod. Phys. Lett. A. - 1999. - V. 14. - P. 223-230.

77. Selyugin $O$. Models of parton distributions and the description of form factors of nucleon // Phys. Rev. D. - 2014. - V. 89, no. 9. - P. 093007. - arXiv:1404.2702 [hep$\mathrm{ph}]$.

78. Galynskii M., Kuraev E. Alternative way to understand the unexpected results of the JLab polarization experiments to measure the Sachs form factors ratio // Phys. Rev. D. - 2014. - V. 89, no. 5. - P. 054005. - arXiv:1312.3742 [hep-ph].

79. Predazzi E., Selyugin O.V. Behavior of the hadron potential at large distances and properties of the hadron spin flip amplitude // Eur. Phys. J. A. - 2002. - V. 13. P. 471-475. - arXiv:hep-ph/0111367.

80. Cudell J., Predazzi E., Selyugin O.V. High-energy hadron spin flip amplitude at small momentum transfer and new $\mathrm{A}(\mathrm{N})$ data from RHIC // Eur. Phys. J. A. 2004. - V. 21. - P. 479-486. - arXiv:hep-ph/0401040.

81. Whalley $M$. The HEPDATA database: The DURHAM-RAL database project // Workshop on Detector and Event Simulation in High-energy Physics (MC '91). 1991. - P. 139-147.

82. Kramer S., Ayres D., Cohen D.H., Diebold R., Pawlicki A., Wicklund A. Polarization Parameter for Nucleon-Nucleon Elastic Scattering at 11.8-GeV/c // Phys. Rev. D. - 1978. - V. 17. - P. 1709.

83. Antille J., Dick L., Werlen M., Gonidec A., Kuroda K., Michalowicz A., PerretGallix D., Crabb D., Kyberd P., Salmon G. Measurement of the Polarization Parameter in $24-\{\mathrm{GeV}\} / c P P$ Elastic Scattering at Large Momentum Transfers // Experientia Suppl. - 1981. - V. 38. 
84. Kline $R$., others. POLARIZATION PARAMETERS AND ANGULAR DISTRIBUTIONS IN PI+- P ELASTIC SCATTERING AT 100-GEV/C AND IN P P ELASTIC SCATTERING AT 100-GEV/C AND 300-GEV/C // Phys. Rev. D. 1980. - V. 22. - P. 553-572.

85. Fidecaro G., others. Measurement of the Differential Cross-section and of the Polarization Parameter in $p p$ Elastic Scattering at $200-\{\mathrm{GeV}\} / c / /$ Phys. Lett. B. 1981. - V. 105. - P. 309-314.

86. Selyugin $O$. The energy dependence of the diffraction minimum in the elastic scattering and new LHC data // Nucl. Phys. A. - 2017. - V. 959. - P. 116-128. arXiv:1609.08847.

87. O'Fallon J., others. Spin Spin Interactions in High p-Transverse**2 Elastic p p Scattering // Phys. Rev. Lett. - 1977. - V. 39. - P. 733.

88. Lin A., others. Energy Dependence of Spin Spin Forces in 90-degrees (Center-ofmass) Elastic $p p$ Scattering // Phys. Lett. B. - 1978. - V. 74. - P. 273-276.

89. Crabb D., others. High precision measurement of A in large $\mathrm{P}(\mathrm{T})^{* *} 2$ spin polarized 24-GeV/c proton proton elastic scattering // Phys. Rev. Lett. - 1990. - V. 65. P. 3241-3244.

90. Abramov $V$. Phenomenology of single-spin effects in hadron production at high energies // Phys. Atom. Nucl. - 2009. - V. 72. - P. 1872-1888.

91. Meshkov I. Luminosity of an Ion Collider // Phys. Part. Nucl. - 2019. - V. 50, no. 6. - P. 663-682.

92. Abramov $V$. A New scaling law for analyzing power in hadron production by transversely polarized baryons // Eur. Phys. J. C. - 2000. - V. 14. - P. 427-441. arXiv:hep-ph/0110152.

93. Abramov V.V. Universal scaling behavior of the transverse polarization for inclusively produced hyperons in hadron hadron collisions. - 2001. - 11. - arXiv:hep$\mathrm{ph} / 0111128$.

94. Baranov $S$. On the production of doubly flavored baryons in $\mathrm{p} \mathrm{p}$, e p and gamma gamma collisions // Phys. Rev. D. - 1996. - V. 54. - P. 3228-3236.

95. Abramov V. Nuclear Effects in the Polarization Phenomena // 15th Workshop on High Energy Spin Physics / Ed. by A.V. Efremov, S.V. Goloskokov. - 2014. P. 17-20.

96. Abramov $V$. On the dependence of the single-spin asymmetry of charged pions on kinematical variables // Phys. Atom. Nucl. - 2007. - V. 70. - P. 2103-2112.

97. Abramov $V$. Single spin effects in collisions of hadrons and heavy ions at high energy // 12th Workshop on High Energy Spin Physics. - 2007. - 11. - P. 1316. - arXiv:0711.0323 [hep-ph]. 
98. Abramov V. Microscopic Stern-Gerlach Effect and Thomas Spin Precession as an Origin of the SSA // 13th Workshop on High Energy Spin Physics. - 2009. - 10. arXiv:0910.1216 [hep-ph].

99. Abramov V. Single-spin physics: Experimental trends and their origin // J. Phys. Conf. Ser. - 2011. - V. 295. - P. 012086.

100. Abramov $V$. An explanation of the new polarization data in the framework of effective color field model // 14th Workshop on High Energy Spin Physics / Ed. by A.V. Efremov, S.V. Goloskokov. - 2011. - P. 21-26.

101. Abramov V. Polarization phenomena in hadronic reactions // Phys. Part. Nucl. 2014. - V. 45. - P. 62-65.

102. Abramov V. Single-spin asymmetry in pp and pA-collisions // J. Phys. Conf. Ser. 2016. - V. 678, no. 1. - P. 012039.

103. Abramov $V$. On the A-dependence of the neutron single-spin asymmetry in pAcollisions // J. Phys. Conf. Ser. - 2017. - V. 938, no. 1. - P. 012038.

104. Abramov V. Polarization of cascade hyperons and antihyperons // J. Phys. Conf. Ser. - 2020. - V. 1435, no. 1. - P. 012001.

105. Abramov V., others. Single-spin asymmetry for charged hadrons produced in proton nucleus collisions at $40-\mathrm{GeV}$ for c.m. production angles in the range 40-degrees to 79-degrees // Phys. Atom. Nucl. - 2007. - V. 70. - P. 1515-1526.

106. Lee J. et al. [BRAHMS Collaboration] Cross-sections and Single Spin Asymmetries of Identified Hadrons in $\mathrm{p}$ uparrow $+\mathrm{p}$ at $\mathrm{s}^{* *}(1 / 2)=200-\mathrm{GeV} / / 17$ th International Workshop on Deep-Inelastic Scattering and Related Subjects. - 2009. - 8. P. 196. - arXiv:0908.4551 [hep-ex].

107. Ho P., others. Production polarization and magnetic moment of anti-XI+ antihyperons produced by $800-\mathrm{GeV} / \mathrm{c}$ protons // Phys. Rev. Lett. - 1990. - V. 65. P. 1713-1716.

108. Felix J. et al. [E766 Collaboration] Inclusive $\bar{\Lambda}$ polarization in pp collisions at 27 GeV // Adriatico Research Conference on Trends in Collider Spin Physics / Ed. by A. Penzo. Y. Onel, N. Paver. - 1997. - P. 231-234.

109. Bellwied R. et al. [E896 Collaboration] The measurement of transverse polarization of Lambda hyperons in relativistic heavy ion collisions // Nucl. Phys. A. - 2002. V. 698. - P. 499-502.

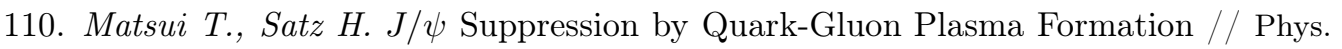
Lett. B. - 1986. - V. 178. - P. 416-422.

111. Zyla P.A. et al. [Particle Data Group Collaboration] Review of Particle Physics // PTEP. - 2020. - V. 2020, no. 8. - P. 083 C01.

112. Uzikov $Y$. - Private communication. 
113. Pancheri G., Srivastava $Y$. Introduction to the physics of the total cross-section at LHC: A Review of Data and Models // Eur. Phys. J. C. - 2017. - V. 77, no. 3.P. 150. - arXiv:1610.10038.

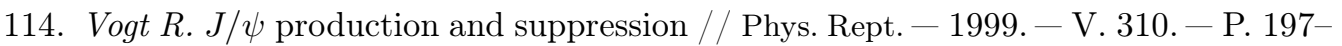
260 .

115. Maltoni F., others. Analysis of charmonium production at fixed-target experiments in the NRQCD approach // Phys. Lett. B. - 2006. - V. 638. - P. 202-208.arXiv:hep-ph/0601203.

116. Isgur N., Wise M.B. Weak Decays of Heavy Mesons in the Static Quark Approximation // Phys. Lett.B. - 1989. - V. 232. - P. 113-117.

117. Brodsky S., Chudakov E., Hoyer P., Laget J. Photoproduction of charm near threshold // Phys. Lett. B. - 2001. - V. 498. - P. 23-28. - arXiv:hep-ph/0010343.

118. Craigie N. Lepton and Photon Production in Hadron Collisions // Phys. Rept. 1978. - V. 47. - P. 1-108.

119. Antipov Y., Bessubov V., Budanov N., Bushnin Y., Denisov S., Gorin Y., Lebedev A., Lednev A., Mikhailov Y., Petrukhin A., Polovnikov S., Roinishvili V., Selesnev

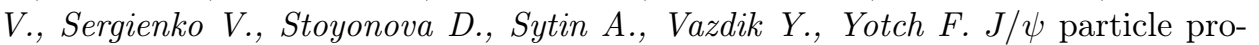
duction by $70 \mathrm{GeV} /$ c protons // Physics Letters B. - 1976. - V. 60, no. 3. - P. 309 - 312. - URL: http://www.sciencedirect.com/science/article/pii/0370269376903087.

120. Vogt R., Brodsky S.J., Hoyer P. Systematics of charm production in hadronic collisions // Nucl. Phys. B. - 1992. - V. 383. - P. 643-684.

121. Lourenço C., Wöhri H. Heavy-flavour hadro-production from fixed-target to collider energies // Physics Reports. - 2006. - Oct. - V. 433, no. 3. - P. 127-180. - URL: http://dx.doi.org/10.1016/j.physrep.2006.05.005.

122. Rekalo M.P., Tomasi-Gustafsson E. Threshold open charm production in nucleonnucleon collisions // Eur. Phys. J.A. - 2003. - V. 16. - P. 575-581. - arXiv:nuclth/0210029.

123. Sibirtsev A., Cassing W. Rho meson production and decay in proton nucleus collisions // Nucl. Phys. A. - 1998. - V. 629. - P. 717-738. - arXiv:nucl-th/9712009.

124. Blobel V. et al. [Bonn-Hamburg-Munich Collaboration] Observation of Vector Meson Production in Inclusive p p Reactions // Phys. Lett. B. - 1974. - V. 48. P. $73-76$.

125. Rustamov A et al. [HADES Collaboration] Inclusive meson production in 3.5-GeV p p collisions studied with the HADES spectrometer // AIP Conf. Proc. - 2010. V. 1257, no. 1. - P. 736-740.

126. Machavariani $A$. On the $\rho^{o}$-meson production in the inclusive proton-proton collision. - 2017. - 12. - arXiv:1712.06395. 
127. Albrow M. et al. [British-French-Scandinavian Collaboration] Inclusive $\rho^{0}$ Production in $p p$ Collisions at the CERN ISR // Nucl. Phys. B. - 1979. - V. 155.P. 39-51.

128. Kuraev E.A., Kokoulina E.S., Tomasi-Gustafsson E. Hard light meson production in (anti)proton-hadron collisions and charge-exchange reactions // Phys. Part. Nucl. Lett. - 2015. - V. 12, no. 1. - P. 1-7. - arXiv:1306.5169 [hep-ph].

129. Baier V., Fadin V.S., Khoze V.A. Quasireal electron method in high-energy quantum electrodynamics // Nucl. Phys. B. - 1973. - V. 65. - P. 381-396.

130. Akhiezer A.I., Beretsetskij V.B. Quantum Electrodynamics. - Moscow : Nauka, 1981.

131. Baier V., Fadin V.S., Katkov V.M. Emission of relativistic electrons. - Moscow : Atomizdat, 1973. - In Russian.

132. Frankfurt L., Sargsian M., Strikman M. Recent observation of short range nucleon correlations in nuclei and their implications for the structure of nuclei and neutron stars // Int. J. Mod. Phys. A. - 2008. - V. 23. - P. 2991-3055.arXiv:0806.4412 [nucl-th].

133. Hen O., Miller G., Piasetzky E., Weinstein L. Nucleon-Nucleon Correlations, Shortlived Excitations, and the Quarks Within // Rev. Mod. Phys. - 2017. - V. 89, no. 4. - P. 045002. - arXiv:1611.09748.

134. Frankfurt L., Piasetsky E., Sargsian M., Strikman M. Probing short range nucleon correlations in high-energy hard quasielastic p d reactions // Phys. Rev. C. - 1995. V. 51. - P. 890-900. - arXiv:nucl-th/9405003.

135. Frankfurt L., Piasetzky E., Sargsian M., Strikman M. On the possibility to study color transparency in the large momentum transfer exclusive d (p, $2 \mathrm{p}) \mathrm{n}$ reaction // Phys. Rev. C. - 1997. - V. 56. - P. 2752-2766. - arXiv:hep-ph/9607395.

136. Frankfurt L., Sargsian M., Strikman M. Feynman graphs and Gribov-Glauber approach to high-energy knockout processes // Phys. Rev. C. - 1997. - V. 56. P. 1124-1137. - arXiv:nucl-th/9603018.

137. Farrar G., Liu H., Frankfurt L., Strikman M. Transparency in Nuclear Quasiexclusive Processes with Large Momentum Transfer // Phys. Rev. Lett. - 1988. V. 61. - P. 686-689.

138. Frankfurt L.L., Strikman M.I. Hard Nuclear Processes and Microscopic Nuclear Structure // Phys. Rept. - 1988. - V. 160. - P. 235-427.

139. Granados C.G., Sargsian M.M. Quark Structure of the Nucleon and Angular Asymmetry of Proton-Neutron Hard Elastic Scattering // Phys. Rev. Lett. - 2009. - V. 103. - P. 212001. - arXiv:0907.2269 [hep-ph].

140. Frankfurt L., Lee T., Miller G., Strikman M. Chiral transparency // Phys. Rev. C. - 1997. - V. 55. - P. 909-916. - arXiv:nucl-th/9608059. 
141. Matveev V., Muradian R., Tavkhelidze A. Automodellism in the large-angle elastic scattering and structure of hadrons // Lett. Nuovo Cim. - 1973. - V. 7. - P. 719 723 .

142. Brodsky S.J., Farrar G.R. Scaling Laws at Large Transverse Momentum // Phys. Rev. Lett. - 1973. - V. 31. - P. 1153-1156.

143. Bizard G., others. EXPERIMENTAL STUDY AND BARYONIC EXCHANGE INTERPRETATION OF THE REACTION H-2 (D, N) HE-3 IN THE INTERMEDIATE-ENERGY REGION // Phys. Rev. C. - 1980. - V. 22. - P. 1632 1637.

144. Uzikov $Y$. Indication of asymptotic scaling in the reactions $\mathrm{dd} \rightarrow \mathrm{p} 3-\mathrm{H}, \mathrm{dd} \rightarrow$ n 3-He and pd $\rightarrow$ pd // JETP Lett. - 2005. - V. 81. - P. 303-306. - arXiv:hep$\mathrm{ph} / 0503185$.

145. Terekhin A., Ladygin V., Gurchin Y., Isupov A., Kurilkin A., Kurilkin P., Ladygina N., Piyadin S., Reznikov S., Khrenov A. Differential Cross Section for Elastic Deuteron-Proton Scattering at the Energy of $700 \mathrm{MeV}$ per Nucleon // Phys. Atom. Nucl. - 2017. - V. 80, no. 6. - P. 1061-1072.

146. Terekhin A., others. The differential cross section in deuteron-proton elastic scattering at 500, 750 and $900 \mathrm{MeV} /$ nucleon // Eur. Phys. J. A. - 2019. - V. 55, no. 8. P. 129

147. Uzikov Y.N. Search for scaling onset in exclusive reactions with the lightest nuclei // Eur. Phys. J. A. - 2016. - V. 52, no. 8. - P. 243. - arXiv:1601.05280.

148. Abbott D. et al. [JLAB t(20) Collaboration] Measurement of tensor polarization in elastic electron deuteron scattering at large momentum transfer // Phys. Rev. Lett. - 2000. - V. 84. - P. 5053-5057. - arXiv:nucl-ex/0001006.

149. Carbonell J., Desplanques B., Karmanov V., Mathiot J. Explicitly covariant light front dynamics and relativistic few body systems // Phys. Rept. - 1998. - V. 300. P. 215-347. - arXiv:nucl-th/9804029.

150. Brodsky S.J., Hiller J.R. Universal properties of the electromagnetic interactions of spin one systems // Phys. Rev. D. - 1992. - V. 46. - P. 2141-2149.

151. Gutsche T., Lyubovitskij V.E., Schmidt I., Vega A. Nuclear physics in soft-wall AdS/QCD: Deuteron electromagnetic form factors / / Phys. Rev. D. - 2015. - V. 91, no. 11. - P. 114001. - arXiv:1501.02738.

152. Gutsche T., Lyubovitskij V.E., Schmidt I. Deuteron electromagnetic structure functions and polarization properties in soft-wall AdS/QCD // Phys. Rev. D. - 2016. V. 94, no. 11. - P. 116006. - arXiv:1607.04124.

153. Bochna C. et al. [E89-012 Collaboration] Measurements of deuteron photodisintegration up to 4.0-GeV // Phys. Rev. Lett. - 1998. - V. 81. - P. 4576-4579.arXiv:nucl-ex/9808001. 
154. Schulte E., others. Measurement of the high energy two-body deuteron photodisintegration differential cross-section // Phys. Rev. Lett. - 2001. - V. 87. - P. 102302.

155. Wijesooriya K. et al. [Jefferson Lab Hall A Collaboration] Polarization measurements in high-energy deuteron photodisintegration // Phys. Rev. Lett. - 2001. V. 86. - P. 2975-2979.

156. Brodsky S.J., Lepage G. Helicity Selection Rules and Tests of Gluon Spin in Exclusive QCD Processes // Phys. Rev. D. - 1981. - V. 24. - P. 2848.

157. Kurilkin P., others. Measurement of the vector and tensor analyzing powers for dpelastic scattering at $880 \mathrm{MeV} / /$ Phys. Lett. B. - 2012. - V. 715. - P. 61-65.arXiv:1207.3509 [nucl-ex].

158. Kurilkin P., others. Investigation of the angular dependence of the analyzing powers in the deuteron-proton elastic scattering at the nuclotron // Phys. Part. Nucl. Lett. 2011. - V. 8. - P. 1081-1083.

159. Ladygin $V$., others. Tensor analyzing power $\mathrm{A}(\mathrm{yy})$ in deuteron inclusive breakup on hydrogen and carbon at $9-\mathrm{GeV} / \mathrm{c}$ and large proton transverse momenta // Phys. Lett. B. - 2005. - V. 629. - P. 60-67.

160. Afanasiev S., others. // Phys. Lett. B. - 1998. - V. 434. - P. 21-25.

161. Blokhintsev D. On the Fluctuations of Nuclear Matter // Sov. Phys. JETP. 1958. - V. 5. - P. 995-999.

162. Efremov A. On the Mechanism of Hadron Cumulative Production on Nucleus // Sov. J. Nucl. Phys. - 1976. - V. 24. - P. 633.

163. Lehman E. Can We See a Six Quark Component of the Deuteron in Inclusive Pion Production? // Phys. Lett. B. - 1976. - V. 62. - P. 296-298.

164. Burov V.V., Lukyanov V.K., Titov A.I. Large Momentum Pion Production in Proton Nucleus Collisions // Phys. Lett. - 1977. - V. 67B. - P. 46-48.

165. Efremov A. Quark-Parton Picture of the Cumulative Production // Fiz. Elem. Chast. Atom. Yadra. - 1982. - V. 13. - P. 613-634.

166. Burov V., Lukyanov V., Titov A. Multi-Quark Systems in Nuclear Processes // Fiz. Elem. Chast. Atom. Yadra. - 1984. - V. 15. - P. 1249-1295.

167. Frankfurt L.L., Strikman M.I. High-Energy Phenomena, Short Range Nuclear Structure and QCD // Phys. Rept. - 1981. - V. 76. - P. 215-347.

168. Alvioli M., Ciofi degli Atti C., Kaptari L.P., Mezzetti C.B., Morita H., Scopetta S. Universality of nucleon-nucleon short-range correlations: two-nucleon momentum distributions in few-body systems // Phys. Rev. C. - 2012. - V. 85. - P. 021001.arXiv:1112.2651 [nucl-th]. 
169. Matveev V.A., Sorba P. Quark Analysis of Multi - Baryonic Systems // Nuovo Cim. A. $-1978 .-$ V. $45 .-$ P. 257.

170. Matveev V.A., Sorba P. Is Deuteron a Six Quark System? // Lett. Nuovo Cim.1977. - V. 20. - P. 435.

171. Conception of Fluctuons and High Momentum Transfer by Complex Systems / D.I. Blokhintsev, A.V. Efremov, V.K. Lukyanov, A.I. Titov // 5th International Seminar on High-Energy Physics Problems: Multiparticle Production and Limiting Fragmentation of Nuclei. - 1978. - 1. - P. 288-299.

172. Efremov A. Polarization in High $p_{T}$ and Cumulative Hadron Production // Sov. J. Nucl. Phys. - 1978. - V. 28. - P. 83.

173. Burov V., Lukyanov V., Reznik B., Titov A. Nuclear Density Fluctuations and Electron Scattering at High-energy // Sov. J. Nucl. Phys. - 1978. - V. 28. - P. 162.

174. Smirnov Y., Chuvilsky Y. Isobaric Component of the Deuteron in the Quark Model // J. Phys. G. - 1978. - V. 4. - P. L1-L4.

175. Mulders P.J., Aerts A.T.M., De Swart J.J. Multi - Quark States. 3. Q**6 Dibaryon Resonances // Phys. Rev. D. - 1980. - V. 21. - P. 2653.

176. Obukhovsky I., Neudachin V., Smirnov Y., Chuvilsky Y. Symmetry of Six Quark System and Color Magnetic Attraction of Nucleons // Yad. Fiz. - 1980. - V. 31. P. 516-519.

177. Pirner H.J., Vary J.P. Deep Inelastic electron Scattering and the Quark Structure of He-3 // Phys. Rev. Lett. - 1981. - V. 46. - P. 1376-1379.

178. Carlson C., Havens T. Quark Distributions in Nuclei // Phys. Rev. Lett. - 1983.V. 51. - P. 261.

179. Efremov A., Kaidalov A., Kim V., Lykasov G., Slavin N. Cumulative Hadron Production in Quark Models of Flucton Fragmentation / Sov. J. Nucl. Phys. - 1988. V. 47.- P. 868 .

180. Kaptar L.P., Titov A.I., Umnikov A.Y. Nuclear effects and nonnucleon degrees of freedom in deep inelastic scattering on nuclei. (In Russian) // Fiz. Elem. Chast. Atom. Yadra. - 1991. - V. 22. - P. 839-881.

181. Leksin $G$. Elastic and quasielastic scattering of $660-\mathrm{MeV}$ protons by deuterons // Sov. Phys. JETP. - 1957. - V. 5. - P. 371-377.

182. Azhgirei L., Vzorov I., Zrelov V., Mescheriakov M., others. Knockout of Deuterons from Li, Be, C and O Nuclei by Protons of Energy $675 \mathrm{MeV} / /$ Sov. Phys. JETP. 1958. - V. 6. - P. 912-919.

183. Baldin A.M. et al.. The Observation of High Energy Pions in Interactions of Relativistic Deuterons with Nuclei // AIP Conf. Proc. - 1971. - V. 2. - P. 131-139. 
184. Baldin A., Giordenescu N., Ivanova L., Moroz N., Povtoreiko A., Radomanov V., Stavinsky V., Zubarev V. An Experimental Investigation of Cumulative Meson Production // Sov. J. Nucl. Phys. - 1975. - V. 20. - P. 629-634.

185. Kim V. QCD Evolution of Nuclear Structure Functions at Large X: EMC Effect and Cumulative Processes // Phys. Part. Nucl. Lett. - 2018. - V. 15, no. 4. - P. 384-386.

186. Boyarinov S., et al. Production of Cumulative $K^{-}$Mesons on Protons at $10 \mathrm{GeV}$ Energy // Sov. J. Nucl. Phys. - 1989. - V. 50. - P. 996-1000.

187. Boyarinov S., et al. The Cumulative production of anti-protons by $10 \mathrm{GeV}$ protons bombarding nuclear targets // Sov. J. Nucl. Phys. - 1991. - V. 54. - P. 71-74.

188. Andrianov A., Andrianov V., Espriu D. Chiral Imbalance in QCD and its consequences // EPJ Web Conf. - 2016. - V. 125. - P. 01009.

189. Efremov A., Kim V., Lykasov G. Hard Hadron-Nucleus Processes and Multi-Quark Configurations in Nuclei // Sov. J. Nucl. Phys. - 1986. - V. 44. - P. 151.

190. Braun M., Vechernin V. Nuclear structure functions and particle production in the cumulative region in the parton model // Nucl. Phys. B. - 1994. - V. 427.P. 614-640.

191. Matveev V., Muradyan R., Tavkhelidze A. Current commutators, vector dominance and self-similarity in strong-interaction lepton pair production // Proc. Int. Seminar Vector mesons and Electromagnetic Interactions 23-26 September 1969, Dubna.1969. - V. 690923. - P. 109-136.

192. Matveev V., Muradian R., Tavkhelidze A. Muon Pair production in Strong Interactions and the Asymptotic Sum Rules. - SLAC-TRANS-0098, JINR-P2-4543, Dubna - June, 1969.

193. Drell S., Yan T.M. Massive Lepton Pair Production in Hadron-Hadron Collisions at High-Energies // Phys. Rev. Lett. - 1970. - V. 25. - P. 316-320. - [Erratum: Phys.Rev.Lett. 25, 902 (1970)].

194. Zotov N., Saleev V., Tsarev V. Cumulative Production of Direct Photons and Lepton Pairs in the Model of Hard Collisions // Yad. Fiz. - 1985. - V. 41. - P. 16351641.

195. Harindranath A., Vary J. Quark Cluster Model Predictions for the Nuclear \{DrellYan\} Process // Phys. Rev. D. - 1986. - V. 34. - P. 3378-3384.

196. Anselmino M., Predazzi E., Ekelin S., Fredriksson S., Lichtenberg D. Diquarks // Rev. Mod. Phys. - 1993. - V. 65. - P. 1199-1234.

197. Barabanov M.Y., others. Diquark correlations in hadron physics: Origin, impact and evidence // Prog. Part. Nucl. Phys. - 2021. - V. 116. - P. 103835. arXiv:2008.07630. 
198. Laperashvili L. V. Dynamical Role of Diquark in Proton Inclusive Prodction // Yad. Fiz. - 1982. - V. 35. - P. 742-747.

199. Larsson T.I. Contribution ofrom Diquarks to the $\mathrm{p} / \mathrm{pi}+$ Ratio at Large $\mathrm{pT}$ in $\mathrm{pi}-\mathrm{p}$ Collisions // Phys. Rev. D. - 1984. - V. 29. - P. 1013-1016.

200. Breakstone A. et al. [ABCDHW Collaboration] A Diquark Scattering Model for High $p_{T}$ Proton Production in $p p$ Collisions at the ISR // Z. Phys. C. - 1985.V. 28. - P. 335 .

201. Kim V. Diquarks as a Source of Large $p_{T}$ Baryons in Hard Nucleon Collisions // JINR-E2-87-75 (1987) Dubna.

202. Kim V. Diquarks and Dynamics of Large $p_{T}$ Baryon Production // Mod. Phys. Lett. A. - 1988. - V. 3. - P. 909-916.

203. Antreasyan D., Cronin J.W., Frisch H.J., Shochet M.J., Kluberg L., Piroue P.A., Sumner R.L. Production of Hadrons at Large Transverse Momentum in 200-GeV, 300-GeV and 400-GeV p p and p n Collisions // Phys. Rev. D. - 1979. - V. 19. P. $764-778$.

204. Abramov V.V., others. Large Transverse Momentum Inclusive Hadron Production in pp Collisions at $70 \mathrm{GeV} / /$ Sov. J. Nucl. Phys. - 1985. - V. 41. - P. 445-451.

205. Kim V.T., Shavrin A.A., Zelenov A.V. Diquark Dynamics and Large $p_{T}$ Baryon Production in $p p$-collisions // Phys. Part. Nucl. Lett. - to appear.

206. Sjostrand, Torbjorn and Ask, Stefan and Christiansen, Jesper R. and Corke, Richard and Desai, Nishita and Ilten, Philip and Mrenna, Stephen and Prestel, Stefan and Rasmussen, Christine O. and Skands, Peter Z.. An introduction to PYTHIA 8.2.

207. Breakstone A. et al. [Ames-Bologna-CERN-Dortmund-Heidelberg-Warsaw Collaboration] Tagging Diquarks by Protons of High Transverse Momentum in $p p$ Collisions at the $\{$ ISR $\} / /$ Z. Phys. C. - 1987. - V. 36. - P. 567.

208. Geist W.M., Drijard D., Putzer A., Sosnowski R., Wegener D. Hadronic Production of Particles at Large Transverse Momentum: Its Relevance to Hadron Structure, Parton Fragmentation and Scattering // Phys. Rept. - 1990. - V. 197. - P. 263374.

209. Landshoff P., Polkinghorne J. p p Elastic scattering at large momentum transfer // Phys. Lett. B. - 1973. - V. 44. - P. 293-295.

210. Landshoff P. Model for elastic scattering at wide angle // Phys. Rev. D. - 1974. V. 10. - P. 1024-1030.

211. Landshoff P., Pritchard D. Higher Order $\{$ QCD $\}$ Corrections to Exclusive Processes: The Multiple Scattering Mechanism // Z. Phys. C. - 1980. - V. 6. - P. 69. 
212. Goebel C., Halzen F., Scott D. Double \{Drell-Yan\} Annihilations in Hadron Collisions: Novel Tests of the Constituent Picture // Phys. Rev. D. - 1980. - V. 22. P. 2789.

213. Paver N., Treleani D. Multi - Quark Scattering and Large $p_{T}$ Jet Production in Hadronic Collisions // Nuovo Cim. A. - 1982. - V. 70. - P. 215.

214. Paver N., Treleani D. Multiple Parton Processes in the TeV Region // Z. Phys. C. 1985. - V. 28. - P. 187.

215. Mekhfi M. Multiparton Processes: an Application to Double Drell-Yan // Phys. Rev. D. - 1985. - V. 32. - P. 2371.

216. Humpert B., Odorico R. Multiparton Scattering and QCD Radiation as Sources of Four Jet Events // Phys. Lett. B. - 1985. - V. 154. - P. 211.

217. Berger E.L., Jackson C.B., Shaughnessy G. Characteristics and Estimates of Double Parton Scattering at the Large Hadron Collider // Phys. Rev. D. - 2010.V. 81. - P. 014014. - arXiv:0911.5348 [hep-ph].

218. Blok B., Dokshitser Y., Frankfurt L., Strikman M. pQCD physics of multiparton interactions // Eur. Phys. J. C. - 2012. - V. 72. - P. 1963. - arXiv:1106.5533 [hep$\mathrm{ph}]$.

219. Ryskin M.G., Snigirev A.M. Double parton scattering in double logarithm approximation of perturbative QCD // Phys. Rev. D. - 2012. - V. 86. - P. 014018. arXiv:1203.2330 [hep-ph].

220. Diehl M., Ostermeier D., Schafer A. Elements of a theory for multiparton interactions in QCD // JHEP. - 2012. - V. 03. - P. 089. - [Erratum: JHEP 03, 001 (2016)] arXiv:1111.0910 [hep-ph].

221. d'Enterria D., Snigirev A.M. Triple parton scatterings in high-energy protonproton collisions // Phys. Rev. Lett. - 2017. - V. 118, no. 12. - P. 122001. arXiv:1612.05582.

222. Kim H., Kim K., Oka M. Hexaquark picture for $d^{*}(2380) / /$ Phys. Rev. D. - 2020. V. 102, no. 7. - P. 074023. - arXiv:2009.11983.

223. Dremin I.M., Kim V.T. Towards a common origin of the elliptic flow, ridge and alignment // Pisma Zh. Eksp. Teor. Fiz. - 2010. - V. 92. - P. 720.arXiv:1010.0918 [hep-ph].

224. Efremov A., Kim V. Diquarks Role in Large $p_{T}$ Deuteron and H-Dihyperon Production in Hard Nucleon Collisions // JINR-E2-87-74 (1987) Dubna. - 1987.

225. Nakano T. et al. [LEPS Collaboration] Evidence for a narrow $\mathrm{S}=+1$ baryon resonance in photoproduction from the neutron // Phys. Rev. Lett. - 2003. - V. 91. P. 012002. - arXiv:hep-ex/0301020. 
226. Barmin $V$. et al. [DIANA Collaboration] Observation of a baryon resonance with positive strangeness in K+ collisions with Xe nuclei // Phys. Atom. Nucl. - 2003. V. 66. - P. 1715-1718. - arXiv:hep-ex/0304040.

227. Stepanyan $S$. et al. [CLAS Collaboration] Observation of an exotic $\mathrm{S}=+1$ baryon in exclusive photoproduction from the deuteron // Phys. Rev. Lett. - 2003. - V. 91. P. 252001. - arXiv:hep-ex/0307018.

228. Diakonov D., Petrov V., Polyakov M.V. Exotic anti-decuplet of baryons: Prediction from chiral solitons // Z. Phys. A. - 1997. - V. 359. - P. 305-314. - arXiv:hep$\mathrm{ph} / 9703373$.

229. Asratyan A.E., Dolgolenko A.G., Kubantsev M.A. Evidence for formation of a narrow $\mathrm{K} 0(\mathrm{~S})$ p resonance with mass near $1533 \mathrm{-MeV}$ in neutrino interactions // Phys. Atom. Nucl. - 2004. - V. 67. - P. 682-687. - arXiv:hep-ex/0309042.

230. Aleev A.N. et al. [SVD Collaboration] Observation of narrow baryon resonance decaying into $\mathrm{p} \mathrm{K} 0(\mathrm{~S})$ in $\mathrm{pA}$ interactions at $70-\mathrm{GeV} / \mathrm{c}$ with SVD-2 setup // Phys. Atom. Nucl. - 2005. - V. 68. - P. 974-981. - arXiv:hep-ex/0401024.

231. Aleev A. et al. [SVD Collaboration] New analysis on narrow baryon resonance decaying into $\mathrm{pK} 0(\mathrm{~s})$ in $\mathrm{pA}$-interactions at $70-\mathrm{GeV} / \mathrm{c}$ with SVD-2 setup. - 2008. 3. - arXiv:0803.3313 [hep-ex].

232. Danilov M. Experimental review on pentaquarks // Frascati Phys. Ser. - 2005. V. 39. - P. 193-209. - arXiv:hep-ex/0509012.

233. Danilov M., Mizuk R. Experimental review on pentaquarks // Phys. Atom. Nucl. 2008. - V. 71. - P. 605-617. - arXiv:0704.3531 [hep-ex].

234. Azimov Y.I., Goeke K., Strakovsky I. An Explanation why the Theta + is seen in some experiments and not in others // Phys. Rev. D. - 2007. - V. 76. - P. 074013. arXiv:0708.2675 [hep-ph].

235. Jaffe R.L. Perhaps a Stable Dihyperon // Phys. Rev. Lett. - 1977. - V. 38.P. 195-198. - [Erratum: Phys.Rev.Lett. 38, 617 (1977)].

236. Dorokhov A.E., Kochelev N.I. Effects of QCD Vacuum and Stability of H Dihyperon. - 1986. - arXiv:hep-ph/0411362.

237. Barabanov M., Vodopyanov A., Olsen $S$. New research of charmonium over $D \bar{D}$ threshold using the antiproton beam with momentum ranging from 1 to $15 \mathrm{GeV} / \mathrm{c} / /$ Phys. Atom. Nucl. - 2014. - V. 77. - P. 126-130.

238. Barabanov M., Vodopyanov A., Zinchenko A., Olsen S. Perspective Study of Charmonium and Exotics above the $D \bar{D}$ Threshold // Phys. Atom. Nucl. - 2016. - V. 79 , no. 1. - P. $126-129$

239. Jaffe R.L., Wilczek F. Diquarks and exotic spectroscopy // Phys. Rev. Lett. 2003. - V. 91. - P. 232003. - arXiv:hep-ph/0307341. 
240. Maiani L., Piccinini F., Polosa A., Riquer V. Diquark-antidiquarks with hidden or open charm and the nature of X(3872) // Phys. Rev. D. - 2005. - V. 71. P. 014028. - arXiv:hep-ph/0412098.

241. Bashkanov M., Brodsky S.J., Clement H. Novel Six-Quark Hidden-Color Dibaryon States in QCD // Phys. Lett. B. - 2013. - V. 727. - P. 438-442.arXiv:1308.6404 [hep-ph].

242. West J.R., Brodsky S.J., de Teramond G.F., Goldhaber A.S., Schmidt I. QCD Hidden-Color Hexa-diquark in the Central Core of Nuclei. - 2020. - 4. arXiv:2004.14659.

243. Li X., Lu X., Wang B., Sun W., Zong H. Properties of cold dense nuclear matter based on a nonperturbative approach inspired by chiral perturbation theory // Phys. Rev. C. - 2009. - V. 80. - P. 034909.

244. Fantina A., Chamel N., Pearson S., Goriely J. Constraints on the equation of state of cold dense matter from nuclear physics and astrophysics // EPJ Web of Conferences. - 2014. - V. 66. - P. 07005.

245. Matveev V., Sorba P. Quark analysis of multibaryonic systems // IL Nuovo Cim. A. - 1978. - V. 45. - P. 257.

246. Gilman R., Gross F. Electromagnetic structure of the deuteron // J. Phys. G. 2002. - V. 28. - P. R37-R116.

247. Efremov A. On the Mechanism of Hadron Cumulative Production on Nucleus // Sov.J.Nucl.Phys. - 1976. - V. 24. - P. 633.

248. Burov V., Lukianov V., Titov A. Large momentum pion production in proton nucleus collisions and the idea of fluctuons in nuclei // Phys. Lett.B. - 1977. - V. 67. P. 46.

249. Frankfurt L., Strikman M. Hard nuclear processes and microscopic nuclear structure // Phys. Rep. - 1988. - V. 160. - P. 235.

250. Kostenko B., Pribiš J. Possible Observation of Phase Transitions in Two-Nucleon Systems // Proceedings, XXII International Baldin Seminar on High Energy Physics Problems. - V. Baldin ISHEPP XXII. - 2015. - P. 122.

251. Troyan Y.A. Uzkie diprotonniye resonansy // Phys.El. Chast.At Yadr. - 1993. V. 24. - P. 683

252. Baldin A. Diff pp. - Soobsheniia OIYAI 1 - 12397, 1979.

253. Abramov B., Dukhovskoy I., Fedorets V., Khanov A., Krestnikov Y., Krutenkova A., Kulikov V., Matsyuk M., Radkevich I., Starodumov A., Sutormin A. Results of search for narrow diproton structures in the pn $\rightarrow \mathrm{pp} \pi^{-}$reaction at $1.98 \mathrm{GeV} / \mathrm{c} / /$ Z.Phys. C - Particles and Fields. - 1995. - V. 69. - P. 409. 
254. Rijken T.A., Nagels M.M., Yamamoto $Y$. Baryon-Baryon Interactions $S=$ 0, $-1,-2,-3,-4 / /$ Few Body Syst. - 2013. - V. 54, no. 7-10. - P. 801-806.

255. Gal A., Millener D.J. Consistency of $\Lambda \Lambda$ hypernuclear events // Hyperfine Interact. 2012. - V. 210, no. 1-3. - P. 77-82. - arXiv:1111.3831 [nucl-th].

256. Rappold $C$. et al. [HypHI Collaboration] Search for evidence of ${ }_{\Lambda}^{3} n$ by observing $d+\pi^{-}$and $t+\pi^{-}$final states in the reaction of ${ }^{6} \mathrm{Li}+{ }^{12} \mathrm{C}$ at $2 \mathrm{~A} \mathrm{GeV} / /$ Phys. Rev.C. - 2013. - V. 88, no. 4. - P. 041001.

257. Marques F.M., others. The Detection of neutron clusters // Phys. Rev.C. - 2002.V. 65. - P. 044006. - arXiv:nucl-ex/0111001.

258. Tang Y.C., Bayman B.F. Nonexistence of the Tetraneutron // Phys. Rev. Lett.1965. - V. 15. - P. 165-168.

259. Bertulani C.A., Zelevinsky $V$. Is the tetraneutron a bound dineutron dineutron molecule? // J. Phys.G. - 2003. - V. 29. - P. 2431-2437. - arXiv:nucl-th/0212060.

260. Pieper S.C. Can modern nuclear Hamiltonians tolerate a bound tetraneutron? // Phys. Rev. Lett. - 2003. - V. 90. - P. 252501. - arXiv:nucl-th/0302048.

261. Timofeyuk N.K. Do Multineutrons exist? // J. Phys.G. - 2003. - V. 29. - P. L9. arXiv:nucl-th/0301020.

262. Lazauskas R., Carbonell J. Is a physically observable tetraneutron resonance compatible with realistic nuclear interactions? // Phys. Rev.C. - 2005. - V. 72. P. 034003. - arXiv:nucl-th/0507022.

263. Takahashi H., others. Observation of a (Lambda Lambda)He-6 double hypernucleus // Phys. Rev. Lett. - 2001. - V. 87. - P. 212502.

264. Garcilazo H., Valcarce A. Strangeness - 2 Hypertriton // Phys. Rev. Lett. - 2013. V. 110, no. 1. - P. 012503. - arXiv:1212.1369 [nucl-th].

265. Gal A. Comment on recent strangeness -2 predictions // Phys. Rev. Lett. - 2013. V. 110. - P. 179201. - arXiv:1301.1241 [nucl-th].

266. Garcilazo H., Valcarce A. Reply to "Comment on Strangeness -2 hypertriton" // Phys. Rev. Lett. - 2013. - V. 110. - P. 179202. - arXiv:1304.3552 [hep-ph].

267. Filikhin I.N., Gal A. Faddeev-Yakubovsky search for ${ }_{\Lambda \Lambda}^{4} \mathrm{H} / /$ Phys. Rev. Lett. 2002. - V. 89. - P. 172502. — arXiv:nucl-th/0209003.

268. Nemura H., Akaishi Y., Myint K.S. Stochastic variational search for ${ }_{\Lambda \Lambda}^{4} \mathrm{H} / /$ Phys. Rev.C. - 2003. - V. 67. - P. 051001. - arXiv:nucl-th/0211082.

269. Thomas L.H. The Interaction Between a Neutron and a Proton and the Structure of ${ }^{3} \mathrm{H} / /$ Phys. Rev. - 1935. - V. 47. - P. 903-909. 
270. Richard J.M., Fleck S. Limits on the domain of coupling constants for binding $N$ body systems with no bound subsystems // Phys. Rev. Lett. - 1994. - V. 73 . P. 1464-1467. - arXiv:nucl-th/9408017.

271. Moszkowski S., Fleck S., Krikeb A., Theußl L., Richard J.M., Varga K. Borromean binding of three or four bosons // Phys. Rev.A. - 2000. - V. 62. - P. 032504. arXiv:nucl-th/0003026.

272. Thogersen M., Fedorov D.V., Jensen A.S. Universal properties of Efimov physics beyond the scattering length approximation // Phys. Rev.A. - 2008. - V. 78. P. 020501.

273. Richard J.M., Wang Q., Zhao Q. Lightest neutral hypernuclei with strangeness -1 and -2 // Phys. Rev. C. - 2015. - V. 91, no. 1. - P. 014003.arXiv:1404.3473 [nucl-th].

274. Richard J.M., Wang Q., Zhao Q. Possibility of a new neutral hypernucleus ${ }_{\Lambda \Lambda}^{4} n=(n, n, \Lambda, \Lambda) / /$ J. Phys. Conf. Ser. - 2014. - V. 569, no. 1. - P. 012079.arXiv:1408.1323 [nucl-th].

275. Rijken T.A., Nagels M.M., Yamamoto Y. Baryon-baryon interactions: Nijmegen extended-soft-core models // Prog. Theor. Phys. Suppl. - 2010. - V. 185. - P. 1471 .

276. Polinder H., Haidenbauer J., Meissner U.G. Strangeness $S=-2$ baryon-baryon interactions using chiral effective field theory // Phys. Lett.B. - 2007. - V. 653. P. 29-37. - arXiv:0705.3753 [nucl-th].

277. Haidenbauer J., Petschauer S., Kaiser N., Meissner U.G., Nogga A., Weise W. Hyperon-nucleon interaction at next-to-leading order in chiral effective field theory // Nucl. Phys.A. - 2013. - V. 915. - P. 24-58. - arXiv:1304.5339 [nucl-th].

278. Haidenbauer J., Meißner U.G., Petschauer S. Strangeness S $=-2$ baryon-baryon interaction at next-to-leading order in chiral effective field theory // Nucl. Phys. A. - 2016. - V. 954. - P. 273-293. - arXiv:1511.05859.

279. Acharya $S$. et al. [ALICE Collaboration] Study of the $\Lambda-\Lambda$ interaction with femtoscopy correlations in $p p$ and $p$-Pb collisions at the LHC // Phys. Lett.B. - 2019. V. 797. - P. 134822. - arXiv:1905.07209.

280. Gal A., Garcilazo H. Is there a bound ${ }_{\Lambda}^{3} n$ ? // Phys. Lett.B. - 2014. - V. 736. P. 93-97. - arXiv:1404.5855 [nucl-th].

281. Hiyama E., Ohnishi S., Gibson B.F., Rijken T.A. Three-body structure of the $n n \Lambda$ system with $\Lambda N-\Sigma N$ coupling // Phys. Rev.C. $-2014 .-$ V. 89, no. 6.P. 061302. - arXiv:1405.2365 [nucl-th].

282. Garcilazo H., Valcarce A. Nonexistence of a $\Lambda n n$ bound state // Phys. Rev. C. 2014. - V. 89, no. 5. - P. 057001. - arXiv:1507.08061. 
283. Lonardoni D., Gandolfi S., Pederiva F. Effects of the two-body and three-body hyperon-nucleon interactions in $\Lambda$-hypernuclei // Phys. Rev.C. - 2013. - V. 87. P. 041303. - arXiv:1301.7472 [nucl-th].

284. Kaidalov A. Diffractive Production Mechanisms // Phys. Rept. - 1979. - V. 50. P. $157-226$.

285. Kaidalov A., Piskunova O. Inclusive Spectra Of Baryons In The Quark - Gluon Strings Model // Z. Phys.C. - 1986. - V. 30. - P. 145.

286. Uzhinsky $V$. Toward Description of pp and pC Interactions at High Energies: Problems of Fritiof-based Models. - 2014. - arXiv:1404.2026 [hep-ph].

287. Andersson B., Gustafson G., Ingelman G., Sjostrand T. Parton Fragmentation and String Dynamics // Phys. Rept. - 1983. - V. 97. - P. 31-145.

288. Sjostrand T., Mrenna S., Skands P. PYTHIA 6.4 Physics and Manual // JHEP. 2006. - V. 0605. - P. 026.

289. Allison J. et al. [GEANT4 Collaboration] Recent developments in Geant4 // Nucl. Instrum. Meth.A. - 2016. - V. 835. - P. 186-225.

290. Aduszkiewicz A. et al. [NA61/SHINE Collaboration] Measurements of $\pi^{ \pm}, \mathrm{K}^{ \pm}, p$ and $\bar{p}$ spectra in proton-proton interactions at 20,31, 40, 80 and $158 \mathrm{GeV} / \mathrm{c}$ with the NA61/SHINE spectrometer at the CERN SPS // Eur. Phys. J.C. - 2017. V. 77.- P. 671 .

291. Blobel V. et al. [Bonn-Hamburg-Munich Collaboration] Multiplicities, topological cross-sections, and single particle inclusive distributions from $\mathrm{p} p$ interactions at 12-GeV/c and 24-GeV/c // Nucl. Phys.B. - 1974. - V. 69. - P. 454-492.

292. Aguilar-Benitez M. et al. [LEBC-EHS Collaboration] Inclusive particle production in $400-\mathrm{GeV} / \mathrm{c}$ p p interactions // Z. Phys.C. - 1991. - V. 50. - P. 405-426.

293. Alt C. et al. [NA49 Collaboration] Inclusive production of charged pions in $\mathrm{p}+\mathrm{p}$ collisions at $158-\mathrm{GeV} / \mathrm{c}$ beam momentum // Eur. Phys. J.C. - 2006. - V. 45. P. 341-381.

294. Anticic T. et al. [NA49 Collaboration] Inclusive production of protons, anti-protons and neutrons in $\mathrm{p}+\mathrm{p}$ collisions at $158-\mathrm{GeV} / \mathrm{c}$ beam momentum // Eur. Phys. J.C. 2010. - V. 65. - P. 9-63.

295. Anticic T. et al. [NA49 Collaboration] Inclusive production of charged kaons in $\mathrm{p}+\mathrm{p}$ collisions at $158 \mathrm{GeV} / \mathrm{c}$ beam momentum and a new evaluation of the energy dependence of kaon production up to collider energies // Eur. Phys. J.C. - 2010. V. 68. - P. 1-73.

296. Adler $S$, others. Jet properties from dihadron correlations in $\mathrm{p}+\mathrm{p}$ collisions at $\sqrt{s_{N N}}=200 \mathrm{GeV} / /$ Phys.Rev.D. - 2006. - V. 74. - P. 072002. 
297. Aaboud M., others. Measurement of jet fragmentation in $\mathrm{Pb}+\mathrm{Pb}$ and $\mathrm{pp}$ collisions at $\sqrt{s_{N N}}=2.76 \mathrm{TeV}$ with the ATLAS detector at the LHC // Eur.Phys.J.C. - 2017. V. 77. - P. 379 .

298. Aaboud M., others. Measurement of jet fragmentation in $5.02 \mathrm{TeV}$ proton-lead and proton-proton collisions with the ATLAS detector // Nucl. Phys.A. - 2018. - V. 978. - P. 65 .

299. Aad G., others. Measurement of angular and momentum distributions of charged particles within and around jets in $\mathrm{Pb}+\mathrm{Pb}$ and pp collisions at $\sqrt{s_{N N}}=5.02 \mathrm{TeV}$ with the ATLAS detector // Phys.Rev.C. - 2019. - V. 100. - P. 064901.

300. Acharya A., others. Jet fragmentation transverse momentum measurements from di-hadron correlations in $\sqrt{s}=7 \mathrm{TeV}$ pp and $\sqrt{s_{N N}}=5.02 \mathrm{TeV}$ p-Pb collisions $/ /$ JHEP. - 2019. - V. 1903. - P. 169.

301. Cacciari M., Salam G., Soyez G. The anti- $k_{t}$ jet clustering algorithm // JHEP.2008. - V. 0804. - P. 063.

302. Cacciari M., Salam G., Soyez G. FastJet User Manual // Eur. Phys. J.C. - 2012.V. 72. - P. 1896 .

303. Schwinger J. Gauge Invariance and Mass. II // Phys.Rev. - 1962. - V. 128. P. 2425-2429.

304. Chliapnikov P. et al. [Brussels-CERN-Genoa-Mons-Nijmegen-Serpukhov Collaboration] Observation of Direct Soft Photon Production in $K^{+} p$ Interactions at 70 $\mathrm{GeV} / c / /$ Phys. Lett. B. - 1984. - V. 141. - P. 276-280.

305. Schukraft J. et al. [HELIOS Collaboration] Recent results from HELIOS (NA34) on proton-nucleus and nucleus- nucleus reactions // Nuclear PhysicsA. - 1989. - V. 498. - P. 79-92.

306. Botterweck $F$. et al. [NA22 Collaboration] Direct soft photon production in $K^{+} p$ and $\pi^{+} p$ interactions at $250 \mathrm{GeV} / c / /$ Z. PhysicsC. - 1991. - V. 51. - P. 541-548.

307. Banerjee $S$. et al. [SOPHIE/WA83 Collaboration] Observation of direct soft photon production in $\pi^{-}$p interactions at $280-\mathrm{GeV} / c / /$ Phys. Lett.B. - 1993. - V. 305. P. 182-186.

308. Belogianni A., others. Confirmation of a soft photon signal in excess of Q.E.D. expectations in $\pi^{-} p$ interactions at $280 \mathrm{GeV} / c / /$ Phys. Lett.B. - 1997. - V. 408. P. 487-492.

309. Belogianni A. et al. [WA102 Collaboration] Observation of soft photon signal in excess of QED expectations in $p p$ interactions // Phys. Lett.B. - 2002. - V. 548. P. 129-139.

310. Abdallah J. et al. [DELPHI Collaboration] Evidence for an excess of soft photons in hadronic decays of $Z^{0} / /$ Eur. Phys. J.C. - 2006. - V. 47. - P. 273-294. 
311. Abdallah J. et al. [DELPHI Collaboration] Evidence for an excess of soft photons in hadronic decays of $\mathrm{Z}^{0} / /$ Eur. Phys. J.C. - 2010. - V. 67. - P. 343-366.

312. Abdallah J. et al. [DELPHI Collaboration] Observation of the muon inner bremsstrahlung at LEP1 // Eur. Phys. J.C. - 2008. - V. 57. - P. 499-514.

313. Kokoulina E., others. Study of soft photon yield in pp and AA interactions at JINR // EPJ Web Conf. - 2020. - V. 235. - P. 0300384.

314. Van Hove L. The cold quark-gluon plasma and multiparticle production // Ann. of Phys. - 1989. - V. 192. - P. 66-76.

315. Barshay $S$. Anomalous soft photons from a coherent hadronic phase in high-energy collisions // Phys. Lett. - 1989. - V. 227. - P. 279-284.

316. Wong C.Y. Open string QED meson description of the X17 particle and dark matter // J. High Energ. Phys. - 1989. - V. 165.

317. Kokoulina E. Description of pp interactions with very high multiplicity at 70 GeVc // Acta Phys. Polon.B. - 2004. - V. 35. - P. 295-302.

318. Kokoulina E. High multiplicity study and gluon dominance model // Phys. Part. Nucl. Lett. - 2016. - V. 13. - P. 74-78.

319. Begun V.V., Gorenstein M. Bose-Einstein condensation of pions in high multiplicity events // Phys. Lett.B. - 2007. - V. 653. - P. 190-195.

320. Peresunko D. Interferometry of direct photons in $\mathrm{Pb}-208+\mathrm{Pb}-208$ collisions at 158 $A \mathrm{GeV} / /$ J. Phys.G. - 2004. - V. 43. - P. 095103.

321. Koide T., Kodama T. Anisotropy of Low Energy Direct Photons in Relativistic Heavy Ion Collisions // Phys. Lett.B. - 2016. - V. 30. - P. 1065-1068.

322. Krasznahorkay A.J., others. Observation of anomalous internal pair creation in ${ }^{8}$ Be: a possible indication of a light neutral boson // Phys. Rev. Lett. - 2016. - V . 116. - P. 042501

323. Abraamyan K., others. Check of the structure in photon pairs spectra at the invariant mass of about $38 \mathrm{MeV} / c^{2} / /$ EPJ Web Conf. - 2019. - V. 138. - P. 04006.

324. Banerjee D. et al. [NA64 Collaboration] Search for a hypothetical $16.7 \mathrm{MeV}$ gauge boson and dark photons in the NA64 Experiment at CERN // Phys. Rev. Lett. 2018. - V. 20. - P. 0231803.

325. Grupen C., Shwartz B. Particles detectors. - Cambridge University Press. New York, 2011.

326. Larson A., Miller G.A., Strikman M. Pionic Color Transparency // Phys. Rev.C. 2006. - V. 74. - P. 018201. - arXiv:nucl-th/0604022.

327. Larionov A., Strikman M. Color transparency in $\bar{p} d \rightarrow \pi^{-} \pi^{0} p$ reaction // Eur. Phys. J. A. - 2020. - V. 56, no. 1. - P. 21. - arXiv:1909.00379. 
328. Dutta D., Hafidi K., Strikman M. Color Transparency: past, present and future // Prog.Part.Nucl.Phys. - 2013. - V. 69. - P. 1-27.

329. Bass S.A., others. Microscopic models for ultrarelativistic heavy ion collisions // Prog. Part. Nucl. Phys. - 1998. - V. 41. - P. 255-369. - [Prog. Part. Nucl. Phys.41,225(1998)] arXiv:nucl-th/9803035.

330. Cassing W., Bratkovskaya E.L. Hadronic and electromagnetic probes of hot and dense nuclear matter // Phys. Rept. - 1999. - V. 308. - P. 65-233.

331. Buss O., Gaitanos T., Gallmeister K., van Hees H., Kaskulov M., Lalakulich O., Larionov A.B., Leitner T., Weil J., Mosel U. Transport-theoretical Description of Nuclear Reactions // Phys. Rept. - 2012. - V. 512. - P. 1-124.arXiv:1106.1344 [hep-ph].

332. Gallmeister K., Mosel U. Time Dependent Hadronization via HERMES and EMC Data Consistency // Nucl. Phys.A. - 2008. - V. 801. - P. 68-79. - arXiv:nuclth/0701064.

333. Strikman M., Tverskoy M.G., Zhalov M.B. Soft neutron production in DIS: A Window to the final state interactions // Phys. Lett.B. - 1999. - V. 459. - P. 3742. - arXiv:nucl-th/9806099.

334. Larionov A., Strikman $M$. Slow neutron production as a probe of hadron formation in high-energy $\gamma^{*} A$ reactions // Phys. Rev. C. $-2020 .-$ V. 101, no. 1. P. 014617. - arXiv:1812.08231.

335. CFNS Adhoc Workshop: Target Fragmentation Physics with EIC. - URL: https://indico.bnl.gov/event/9287/.

336. Gallmeister K., Falter T. Space-time picture of fragmentation in PYTHIA/JETSET for HERMES and RHIC // Phys. Lett.B. - 2005. - V. 630. - P. 40-48.arXiv:nucl-th/0502015.

337. Sjostrand T., Mrenna S., Skands P.Z. PYTHIA 6.4 Physics and Manual // JHEP.2006. - V. 05. - P. 026. - arXiv:hep-ph/0603175.

338. Larionov A., Strikman M. Color Transparency and Hadron Formation Effects in High-Energy Reactions on Nuclei // Particles. - 2020. - V. 3, no. 1. - P. 24-38.

339. Kuhn S.E., Chen J.P., Leader E. // Prog.Part.Nucl.Phys. - 2009. - V. 63. - P. 150.

340. Deur A., Brodsky S.J., de Teramond G.F. // Rep.Prog. Phys. - 2019. - V. 82(7).

341. Belitsky A.V., Radyushkin A.V. // Phys.Rept. - 2005. - V. 418. - P. 1-387.

342. Goeke K., Polyakov M.V., Vanderhaeghen M. // Prog.Part.Nucl.Phys. - 2001.V. 47. - P. 401-515.

343. Diehl M. Phys.Rept. - 2003. - V. 388. - P. 41-277. 
344. D'Alesio U., Murgia F. // Prog.Part.Nucl.Phys. - 2008. - V. 61. - P. 394-454.

345. Barone V., Drago A., Ratcliffe P.G. Phys.Rept. - 2002.- V. 359.- P. 1-168.

346. Alekhin S., Melnikov K., Petriello F. // Phys.Rev.D. - 2006. - V. 74. - P. 054033.

347. Bacchetta A., Diehl M., Goeke K., Metz A., Mulders P.J., Schlegel M. // JHEP02. - 2007. - V. 93.

348. Collaboration C. // PoS Dis2019. - 2019. - V. 267.

349. Arnold S., Metz A., Schlegel M. // Phys.Rev. D. - 2009. - V. 79. - P. 034005.

350. Sissakian A., Shevchenko O., Nagaytsev A., Ivanov O. // Phys.Rev. D. - 2005.V. 72. - P. 054027.

351. Sissakian A., Shevchenko O., Nagaytsev A., Ivanov O. // Eur.Phys.J C. - 2009.V. 59.- P. 659-673.

352. Sissakian A., Shevchenko O., Nagaytsev A., Denisov O., Ivanov O. // Eur.Phys.J C. - 2006. - V. 46. - P. 147-150.

353. Sissakian A., Shevchenko O., Ivanov O. 86 // JETP Lett. - 2007. - V. 86.P. 751-755.

354. A. Sissakian O. Shevchenko A.N.O.I. // Physics of Particles and Nuclei. - 2010.V. 41. - P. 64-100.

355. J. C. Collins D.E.S., Sterman G. // Nucl. Phys. B. - 1985. - V. 250. - P. 199-224.

356. Brodsky S.J., Hwang D.S., Schmidt I. // Nucl. Phys. B. - 2002. - V. 642. - P. 344356.

357. Drell S.D., Yan T.M. // Phys.Rev.Lett. - 1970. - V. 25. - P. 316-320.

358. Bodwin G.T. // Phys. Rev. D. - 1985. - V. 31(10). - P. 2616-2642.

359. Collins J.C. // Nuclear Physics B. - 1993. - V. 394. - P. 169-199.

360. Boos E., Bunichev V., Carena M., Wagner C.E.M. // Econf C050318:0213. - 2005.

361. Nojiri M.M. // Phys.Rev. D. - 1995. - V. 51. - P. 6281.

362. Boos E., Martyn H.U., Moortgat-Pick G., Sachwitz M., Sherstnev A., Zerwas P.M. // Eur.Phys. J. C. - 2003. - V. 30. - P. 395.

363. Boos E., Moortgat-Pick G., Martyn H.U., Sachwitz M., Vologdin A. - arXiv:hep$\mathrm{ph} / 0211040$.

364. Savin I.A., Efremov A.V., Peshekhonov D.V., Kovalenko A.D., Teryaev O.V., Shevchenko O.Y., Nagajcev A.P., Guskov A.V., Kukhtin V.V., et al. N.D.T. // Epj Web Conf. - 2015. - V. 85. - P. 02039. 
365. Boos E., Bunichev V., Dubinin M., Dudko L., Edneral V., Ilyin V., Kryukov A., Savrin V., Semenov A., Sherstnev A. // Nucl.Instrum.Meth. A. - arXiv:hep$\mathrm{ph} / 0403113$.

366. Alwall J., et al.. - arXiv:1405.0301[hep-ph].

367. Ball R.D. et al. [NNPDF Collaboration] // Eur. Phys. J. C. - 2017. - V. 77, no. 10. - P. 663. - arXiv:1706.00428.

368. Maltoni F., McElmurry T., Putman R., Willenbrock S. - 2007. - 3. - arXiv:hep$\mathrm{ph} / 0703156$.

369. Olive K., et al. (Particle Data Group). // Chin. Phys.C. - 2014. - V. 38. P. 090001.

370. Nocera E.R. et al. [NNPDF Collaboration] // Nucl. Phys. B. - 2014. - V. 887.P. 276-308. - arXiv:1406.5539 [hep-ph].

371. Sjstrand T., Mrenna S., Skands P. // JHEP05. - 2006. - V. 26.

372. de Favereau J., Delaere C., Demin P., Giammanco A., Lematre V., Mertens A., Selvaggi M. - arXiv:1307.6346[hep-ex].

373. Sirunyan A. et al. [CMS Collaboration] // JINST. - 2018. - V. 13, no. 10.P. P10005. - arXiv:1809.02816.

374. Ade P.A.R. et al. [Planck Collaboration] Planck 2013 Results. I. Overview of Products and Scientific Results // Astronomy \& Astrophysics. - 2014. - V. 571. - P. A1.

375. Majumdar D. Dark Matter: An Introduction. - CRC Press, 2014. - Aug.

376. Clowe D., Bradač M., Gonzalez A.H., Markevitch M., Randall S.W., Jones C., Zaritsky D. A Direct Empirical Proof of the Existence of Dark Matter // The Astrophysical Journal. - 2006. - Aug. - V. 648, no. 2. - P. L109-L113.

377. Buchmueller O., Doglioni C., Wang L.T. Search for Dark Matter at Colliders // Nature Physics. - 2017. - Mar. - V. 13, no. 3. - P. 217-223.

378. Adriani O. et al. [PAMELA Collaboration] The PAMELA Mission: Heralding A New Era in Precision Cosmic Ray Physics // Physics Reports. - 2014. - Nov. - V. 544, no. 4. - P. 323-370.

379. Aguilar M. et al. [AMS Collaboration] First Result from the Alpha Magnetic Spectrometer on the International Space Station: Precision Measurement of the Positron Fraction in Primary Cosmic Rays of $0.5-350 \mathrm{GeV} / /$ Physical Review Letters. 2013. - Apr. - V. 110, no. 14.

380. Aguilar M. et al. [AMS Collaboration] Antiproton Flux, Antiproton-to-Proton Flux Ratio, and Properties of Elementary Particle Fluxes in Primary Cosmic Rays Measured with the Alpha Magnetic Spectrometer on the International Space Station // Physical Review Letters. - 2016. - Aug. - V. 117, no. 9. 
381. Giesen G., Boudaud M., Génolini Y., Poulin V., Cirelli M., Salati P., Serpico P.D. AMS-02 Antiprotons, At Last! Secondary Astrophysical Component and Immediate Implications for Dark Matter // Journal of Cosmology and Astroparticle Physics. 2015. - Sep. - V. 2015, no. 09. - P. 023-023.

382. di Mauro M., Donato F., Goudelis A., Serpico P.D. New Evaluation of the Antiproton Production Cross Section for Cosmic Ray Studies // Physical Review D. 2014. - Oct. - V. 90, no. 8.

383. Donato F., Korsmeier M., Mauro M.D. Prescriptions on Antiproton Cross Section Data for Precise Theoretical Antiproton Flux Predictions // Physical Review D. 2017. - Aug. - V. 96, no. 4.

384. Guskov A., El-Kholy R. On the Possibility to Study Antiproton Production at the SPD Detector at NICA Collider for Dark Matter Search in Astrophysical Experiments // Physics of Particles and Nuclei Letters. - 2019. - may. - V. 16, no. 3.P. 216-223.

385. Alexakhin V., Guskov A., Hayman Z., El-Kholy R., Tkachenko A. On the Study of Antiprotons Yield in Hadronic Collisions at NICA SPD. - 2020. - arXiv:2008.04136.

386. Winkler M.W. Cosmic Ray Antiprotons at High Energies // Journal of Cosmology and Astroparticle Physics. - 2017. - Feb. - V. 2017, no. 02. - P. 048.

387. Aaij R. et al. [LHCb Collaboration] Measurement of Antiproton Production in pHe Collisions at $\mathrm{sNN}=110 \mathrm{GeV} / /$ Physical Review Letters. - 2018. - Nov. - V. 121, no. 22 .

388. Kappl R., Winkler M.W. The Cosmic Ray Antiproton Background for AMS-02 // Journal of Cosmology and Astroparticle Physics. - 2014. - Sep. - V. 2014, no. 09. P. 051 .

389. Savin I., Efremov A., Peshekhonov D., Kovalenko A., Teryaev O., Shevchenko O., Nagajcev A., Guskov A., Kukhtin V., Toplilin N. Spin Physics Experiments at NICA-SPD with Polarized Proton and Deuteron Beams // EPJ Web of Conferences. - 2015. - V. 85. - P. 02039.

390. Meshkov I.N. Luminosity of an Ion Collider // Physics of Particles and Nuclei. 2019. - Nov. - V. 50, no. 6. - P. 663-682.

391. Sjöstrand T., Ask S., Christiansen J.R., Corke R., Desai N., Ilten P., Mrenna S., Prestel S., Rasmussen C.O., Skands P.Z. An Introduction to PYTHIA $8.2 / /$ Computer Physics Communications. - 2015. - Jun. - V. 191. - P. 159-177.

392. Agapov N.N., Kekelidze V.D., Kovalenko A.D., Lednitsky R., Matveev V.A., Meshkov I.N., Nikitin V.A., Potrebennikov Y.K., Sorin A.S., Trubnikov G.V. Relativistic nuclear physics at JINR: from the synchrophasotron to the NICA collider // Phys. Usp. - 2016. - V. 59, no. 4. - P. 383-402. - [Usp. Fiz. Nauk186,no.4,405(2016)]. 
393. Savin I., Efremov A., Peshekhonov D., Kovalenko A., Teryaev O., Shevchenko O., Nagajcev A., Guskov A., Kukhtin V., Toplilin N. Spin Physics Experiments at NICA-SPD with polarized proton and deuteron beams // EPJ Web of Conferences. 2015. - V. 85. - P. 02039. — URL: https://doi.org/10.1051/epjconf/20158502039.

394. Kistryn S., Lang J., Liechti J., Maier T., Muller R., Nessi-Tedaldi F., Simonius M., Smyrski J., Jaccard S., Haeberli W., Sromicki J. Precision measurement of parity nonconservation in proton-proton scattering at $45 \mathrm{MeV} / /$ Phys. Rev. Lett. - 1987. - Apr. - V. 58. - P. 1616-1619. - URL: https://link.aps.org/doi/10.1103/PhysRevLett.58.1616.

395. Lockyer N., Romanowski T.A., Bowman J.D., Hoffman C.M., Mischke R.E., Nagle D.E., Potter J.M., Talaga R.L., Swallow E.C., Alde D.M., Moffett D.R., Zyskind J. Parity nonconservation in proton-nucleus scattering at $6 \mathrm{GeV} / \mathrm{c} / /$ Phys. Rev. D. - 1984. - sep. - V. 30, no. 5. - P. 860-871. - URL: https://link.aps.org/doi/10.1103/PhysRevD.30.860

396. Gardner S., Haxton W.C., Holstein B.R. A New Paradigm for Hadronic Parity Nonconservation and its Experimental Implications // Ann. Rev. Nucl. Part. Sci. 2017. - V. 67. - P. 69-95.

397. Grosnick D.P., Hill D.A., Kasprzyk T., Lopiano D., Ohashi Y., Sheppard J., Shima T., Spinka H., Stanek R., Underwood D.G., Yokosawa A., Bystricky J., Chaumette P., Derégel J., Durand G., Fabre J., Lehar F., de Lesquen A., van Rossum L., Luehring F.C., Miller D.H., Shanahan P., Krueger K.W., Cossairt J.D., Read A.L., Iwatani K., Belikov N.I., Derevschikov A.A., Grachov O.A., Matulenko Y.A., Meschanin A.P., Nurushev S.B., Patalakha D.I., Rykov V.L., Solovyanov V.L., Vasiliev A.N., Akchurin N., Onel Y., Maki T., En'yo H., Funahashi H., Goto Y., Iijima T., Imai K., Itow Y., Makino S., Masaike A., Miyake K., Nagamine T., Saito N., Yamashita S., Takashima R., Takeutchi F., Kuroda K., Michalowicz A., Tanaka N., Salvato G., Villari A., Tamura N., Yoshida T., Corcoran M.D., Cranshaw J., Nessi-Tedaldi F., Nessi M., Nguyen C., Roberts J.B., Skeens J., White J.L., Bravar A., Penzo A., Schiavon P., Pauletta G. Measurement of the differences in the total cross section for antiparallel and parallel longitudinal spins and a measurement of parity nonconservation with incident polarized protons and antiprotons at $200 \mathrm{GeV} / c / /$ Phys. Rev. D. - 1997. - Feb. - V. 55. - P. 1159-1187. URL: https://link.aps.org/doi/10.1103/PhysRevD.55.1159.

398. Koop I., Milstein A., Nikolaev N., Popov A., Salnikov S., Shatunov P., Shatunov $Y$. Strategies for Probing $P$-Parity Violation in Nuclear Collisions at the NICA Accelerator Facility // Phys. Part. Nucl. Lett. - 2020. - V. 17, no. 2. - P. 154-159.

399. Milstein A., Nikolaev N., Salnikov S. Parity violation in proton-proton scattering at high energies // JETP Lett. - 2020. - V. 111, no. 4. - P. 197-200.

400. Milstein A., Nikolaev N., Salnikov S. Parity violation in proton-deuteron scattering // JETP Lett. - 2020. - V. 112, no. 6. - P. 332-336. 
401. Vasserman I., Vorobyov P., Gluskin E., Ivanov P., Kezerashvili G., Koop I., Lysenko A., Mikhailichenko A., Nesterenko I., Perevedentsev E., Polunin A., Serednyakov S., Skrinsky A., Shatunov $Y$. New experiment on the precise comparison of the anomalous magnetic moments of relativistic electrons and positrons // Physics Letters B. - 1987. - V. 187, no. 1. - P. 172-174. - URL: http://www.sciencedirect.com/science/article/pii/0370269387900943.

402. Sitnik I.M., Volkov V.I., Kirillov D.A., Piskunov N.M., Plis Y.A. Precessing deuteron polarization // PEPAN Lett. - 2002. - V. 2. - P. 22.

403. Bagdasarian Z., Bertelli S., Chiladze D., Ciullo G., Dietrich J., Dymov S., Eversmann D., Fanourakis G., Gaisser M., Gebel R., Gou B., Guidoboni G., Hejny V., Kacharava A., Kamerdzhiev V., Lehrach A., Lenisa P., Lorentz B., Magallanes L., Maier R., Mchedlishvili D., Morse W.M., Nass A., Oellers D., Pesce A., Prasuhn D., Pretz J., Rathmann F., Shmakova V., Semertzidis Y.K., Stephenson E.J., Stockhorst H., Ströher H., Talman R., Thörngren Engblom P., Valdau Y., Weidemann C., Wüstner P. Measuring the polarization of a rapidly precessing deuteron beam // Phys. Rev. ST Accel. Beams. - 2014. - May. - V. 17. - P. 052803. - URL: https://link.aps.org/doi/10.1103/PhysRevSTAB.17.052803.

404. Eversmann D. et al. [JEDI Collaboration] New method for a continuous determination of the spin tune in storage rings and implications for precision experiments // Phys. Rev. Lett. - 2015. - V. 115, no. 9. - P. 094801. - arXiv:1504.00635.

405. Guidoboni G. et al. [JEDI Collaboration Collaboration] How to Reach a ThousandSecond in-Plane Polarization Lifetime with $0.97-\mathrm{GeV} / c$ Deuterons in a Storage Ring // Phys. Rev. Lett. - 2016. - Jul. - V. 117. - P. 054801. - URL: https://link.aps.org/doi/10.1103/PhysRevLett.117.054801.

406. Guidoboni G. et al. [JEDI Collaboration Collaboration] Connection between zero chromaticity and long in-plane polarization lifetime in a magnetic storage ring // Phys. Rev. Accel. Beams. - 2018. - Feb. - V. 21. - P. 024201. - URL: https://link.aps.org/doi/10.1103/PhysRevAccelBeams.21.024201.

407. Hempelmann N. et al. [JEDI Collaboration Collaboration] Phase Locking the Spin Precession in a Storage Ring // Phys. Rev. Lett. - 2017. - Jul. - V. 119. P. 014801. - URL: https://link.aps.org/doi/10.1103/PhysRevLett.119.014801.

408. Fimushkin V.V., Kovalenko A.D., Kutuzova L.V., Prokofichev Y.V., Shutov B., Belov A.S., Zubets V.N., Turbabin A.V. Source of polarised deuterons // The European Physical Journal Special Topics. - 2018. - f. - V. 162. - P. 275-280.

409. Vokal S., Kovalenko A., Kondratenko A., Kondratenko M., Mikhailov V., Filatov $Y$., Shimansky S. Program of polarization studies and capabilities of accelerating polarized proton and light nuclear beams at the Nuclotron of the Joint Institute for Nuclear Research // Pisma Fiz. Elem. Chast. Atom. Yadra. - 2009. - V. 2009, no. 149. - P. 81-96. 
410. Lenisa P., Rathmann F., Barion L., Barsov S., Bertelli S., Carassiti V., Ciullo G., Contalbrigo M., Ramusino A.C., Dymov S., Engels R., Eversheim D., Gebel R., Grigoryev K., Haidenbauer J., Hejny V., Jagdfeld H., Kacharava A., Keshelashvili I., Kononov A., Krings T., Kulikov A., Lehrach A., Lorentz B., Lomidze N., Macharashvili G., Malaguti R., Martin S., Merzliakov S., Mikirtychiants S., Nass A., Nikolaev N., Pesce A., Prasuhn D., Semke L., Squerzanti S., Soltner H., Statera M., Steffens E., Ströher H., Tabidze M., Tagliente G., Thörngren-Engblom P., Trusov S., Uzikov Y., Valdau Y., Weidemann C., Wüstner P., Zupranski P. Lowenergy spin-physics experiments with polarized beams and targets at the COSY storage ring // EPJ Techniques and Instrumentation. - 2019. - V. 6, no. 1. - P. 2.

411. Nikolaev N., Rathmann F., Silenko A., Uzikov Y. New approach to search for parityeven and parity-odd time-reversal violation beyond the Standard Model in a storage ring // Phys. Lett. B. - 2020. - V. 811. - P. 135983.

412. Okun L.B. Note concerning CP parity // Yad. Fiz. - 1965. - V. 1. - P. 938-939.

413. Prentki J., Veltman M.J.G. Possibility of CP violation in semistrong interactions // Phys. Lett. - 1965. - V. 15. - P. 88-90.

414. Lee T.D., Wolfenstein L. Analysis of CP Noninvariant Interactions and the K0(1), K0(2) System // Phys. Rev. - 1965. - V. 138. - P. B1490-B1496.

415. Bernreuther W. CP violation and baryogenesis // Lect. Notes Phys. - 2002. - V. 591. - P. 237-293.

416. Koop I., Shatunov Y. The Spin Precession Tune Spread in the Storage Ring // Particle accelerator. Proceedings, 1st EPAC Conference, Rome, Italy, June 7-11, 1988. Vol. 1, 2 / Ed. by S. Tazzari. - Singapore: World Scientific, 1988. - June. - P. 738-739.

417. Glauber R. Cross-sections in deuterium at high-energies // Phys. Rev. - 1955. - V. 100. - P. 242-248.

418. Franco V., Glauber R. High-energy deuteron cross-sections // Phys. Rev. - 1966. V. 142. - P. 1195-1214.

419. Derbenev Ya.S., Morozov V.S., Lin F., Zhang Y., Kondratenko A.M., Kondratenko M.A., Filatov Yu.N. Polarization Preservation and Control in a Figure-8 Ring // Int. J. Mod. Phys. Conf. Ser. - 2016. - V. 40, no. 01. - P. 1660090.

420. Filatov Yu.N., Kondratenko A.M., Kondratenko M.A., Derbenev Ya.S., Morozov V.S. Transparent Spin Method for Spin Control of Hadron Beams in Colliders // Phys. Rev. Lett. - 2020. - V. 124, no. 19. - P. 194801.

421. Filatov Y.N., Kondratenko A.M., Kondratenko M.A., Derbenev Y.S., Morozov V.S., Kovalenko A.D. Spin response function technique in spin-transparent synchrotrons // Eur. Phys. J.C. - 2020. - V. 80, no. 8. - P. 778. 
422. Experimental verification of transparent spin mode in RHIC / V. Morozov, P. Adams, Y. Derbenev, Y. Filatov, H. Huang, He Huang, A. Kondratenko, M. Kondratenko, F. Lin, F. Méot, V. Ptitsyn, W. Schmidke, Y. Zhang // Proc. 10th International Particle Accelerator Conference (IPAC'19), Melbourne, Australia, 19-24 May 2019. - P. 2783-2786.

423. Huang H., Méot F., Ptitsyn V., Ranjbar V., Roser T. Polarization preservation of polarized deuteron beams in the electron ion collider at Brookhaven National Laboratory // Phys. Rev. Accel. Beams. - 2020. - V. 23, no. 2. - P. 021001.

424. Montag C. Polarized Electron and Hadron Beams at eRHIC // PoSP. - 2020. - V STP2019. - P. 009. 